\title{
Dithionite-Involved Multicomponent Coupling for Alkenyl and Alkyl Tertiary Sulfones
}

\author{
Yaping Li, Ming Wang ${ }^{*}$, Xuefeng Jiang
}

\begin{abstract}
Shanghai Key Laboratory of Green Chemistry and Chemical Process, School of Chemistry and Molecular Engineering, East China Normal University, 3663 North Zhongshan Road, Shanghai 200062, P. R. China
\end{abstract}

\section{Supporting Information}

\author{
Table of Contents
}

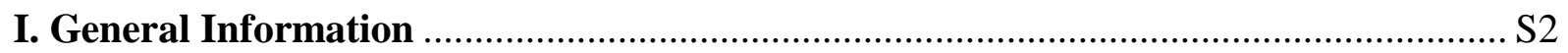

II. General procedure for the alkyl alkyl sulfones synthesis f..................................... S3

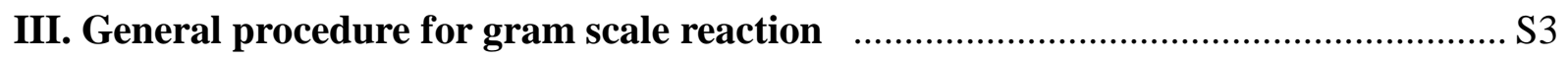

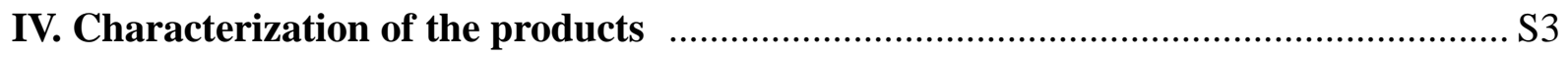

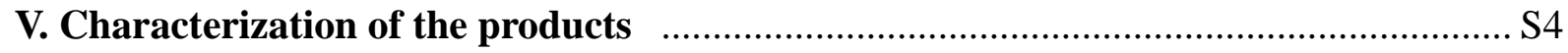

VI. X-Ray crystal structures

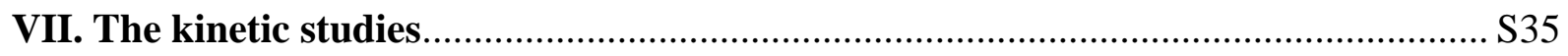

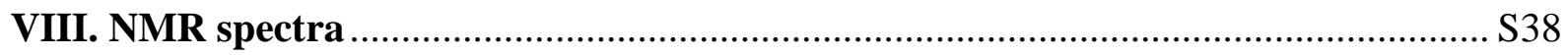




\section{General Information.}

${ }^{1} \mathrm{H}$ and ${ }^{13} \mathrm{C}$ NMR spectra were recorded on $400 \mathrm{MHz}$ NMR spectrometers (Bruker AVANCE) using $\mathrm{CDCl}_{3}$. Chemical shifts are reported in parts per million (ppm). Chemical shifts for protons are reported in parts per million relative to chloroform $(\delta 7.26)$. Chemical shifts for carbon are reported in parts per million relative to chloroform $(\delta 77.0)$. Data are represented as follows: chemical shift, multiplicity $(b r=$ broad, $s=$ singlet, $d=$ doublet, $t=$ triplet, $q=$ quartet, $m=$ multiplet), coupling constants in Hertz $(\mathrm{Hz})$, integration. High-resolution mass spectra were recorded on Bruck microTOF by using ESI method. IR spectra were recorded on TENSOR (27) Series FT-IR 241.Spectrometers. The crystal structures were determined by single crystal X-ray diffraction analysis on Rigaku XtaLAB PRO MM003-DS dual system.

Redox-active ester were prepared adopting the reported procedures. ${ }^{1,2}$

Table S1. Conditions Optimization.

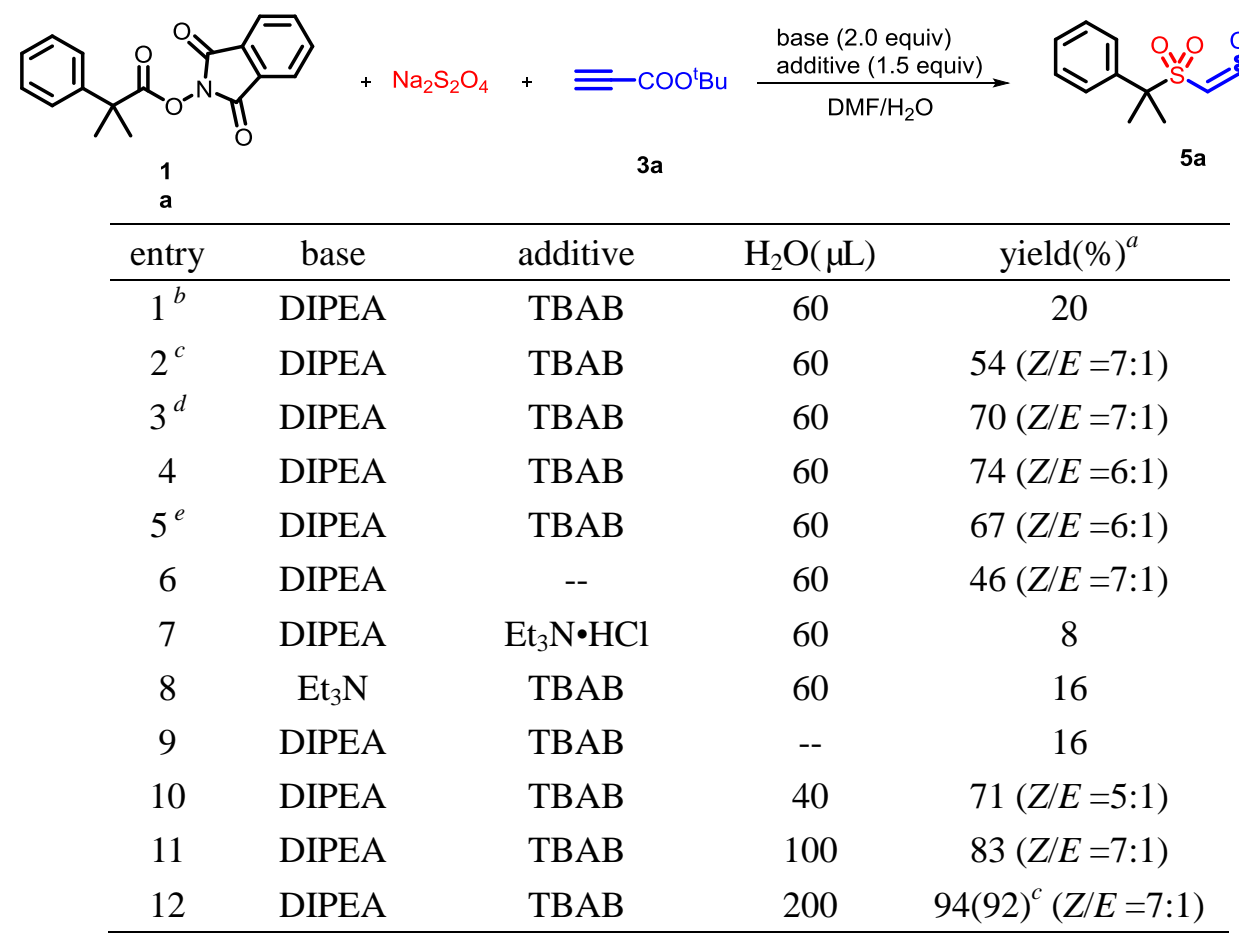

Alkyl-alkenyl sulfones: 1a $(0.2 \mathrm{mmol}), \mathrm{Na}_{2} \mathrm{~S}_{2} \mathrm{O}_{4}(0.4 \mathrm{mmol})$, alkyne $(0.4 \mathrm{mmol})$, DIPEA $(0.4 \mathrm{mmol})$; TBAB $(0.3 \mathrm{mmol})$, DMF: $\mathrm{H}_{2} \mathrm{O}=9: 1(2 \mathrm{~mL}), 40{ }^{\circ} \mathrm{C}, \mathrm{N}_{2}, 15$ h. ${ }^{a} \mathrm{NMR}$ yields. ${ }^{b} 100{ }^{\circ} \mathrm{C} .{ }^{c} 80{ }^{\circ} \mathrm{C} .{ }^{d} 60{ }^{\circ} \mathrm{C} .{ }^{e} \mathrm{rt}$. 


\section{General procedure for the alkyl alkyl sulfones synthesis}

Under a $\mathrm{N}_{2}$ atmosphere, NHPI ester $(0.2 \mathrm{mmol}), \mathrm{Na}_{2} \mathrm{~S}_{2} \mathrm{O}_{4}(52.2 \mathrm{mg}, 0.3$ $\mathrm{mmol})$, olefin Michael acceptor $(0.6 \mathrm{mmol}), \mathrm{Et}_{3} \mathrm{~N} \cdot \mathrm{HCl}(68.8 \mathrm{mg}, 0.5 \mathrm{mmol})$, LiOAc (13.2 mg, $0.2 \mathrm{mmol})$, DMF (2 mL) were added to a Schlenk tube. The reaction was stirred at $100{ }^{\circ} \mathrm{C}$ oil bath for $15 \mathrm{~h}$. The reaction was quenched with water and was extracted with ethyl acetate; the organic layers were combined, dried over $\mathrm{Na}_{2} \mathrm{SO}_{4}$ anhydrous. After evaporation of solvent, the residue was purified by column chromatography to give the corresponding products.

\section{General procedure for the alkyl alkenyl sulfones synthesis}

Under a $\mathrm{N}_{2}$ atmosphere, NHPI ester (0.2 mmol), $\mathrm{Na}_{2} \mathrm{~S}_{2} \mathrm{O}_{4}$ (69.6 mg, 0.4 $\mathrm{mmol})$, alkyne Michael acceptor $(0.4 \mathrm{mmol})$, DIPEA (51.7 $\mathrm{mg}, 0.4 \mathrm{mmol})$, TBAB (97.6 mg, $0.3 \mathrm{mmol})$, solvent $\left(\mathrm{DMF} / \mathrm{H}_{2} \mathrm{O}=9: 1,2 \mathrm{~mL}\right)$ were added to a Schlenk tube. The reaction was stirred at $40{ }^{\circ} \mathrm{C}$ oil bath for $15 \mathrm{~h}$. The reaction was quenched with water and was extracted with ethyl acetate; the organic layers were combined, dried over $\mathrm{Na}_{2} \mathrm{SO}_{4}$ anhydrous. After evaporation of solvent, the residue was purified by column chromatography to give the corresponding products.

\section{General procedure for gram scale reaction}

Under a $\mathrm{N}_{2}$ atmosphere, NHPI ester $1 \mathrm{a}$ (309.5 mg, $\left.1 \mathrm{mmol}\right), \mathrm{Na}_{2} \mathrm{~S}_{2} \mathrm{O}_{4}(261 \mathrm{mg}$, $1.5 \mathrm{mmol})$, tert-butyl acrylate $(435 \mu \mathrm{L}, 3 \mathrm{mmol}), \mathrm{Et}_{3} \mathrm{~N} \cdot \mathrm{HCl}(344 \mathrm{mg}, 2.5 \mathrm{mmol})$, LiOAc (66 mg, $1 \mathrm{mmol}$ ), DMF (10 mL) were added to a Schlenk tube. The reaction was stirred at $100{ }^{\circ} \mathrm{C}$ oil bath for $15 \mathrm{~h}$. The reaction was quenched with water and was extracted with ethyl acetate; the organic layers were combined, dried over $\mathrm{Na}_{2} \mathrm{SO}_{4}$ anhydrous. After evaporation of solvent, the residue was purified by column chromatography to give the corresponding product $4 \mathrm{a}$ in $63 \%$ yield $(196.4 \mathrm{mg})$ as a white solid. 


\section{Characterization of the products}

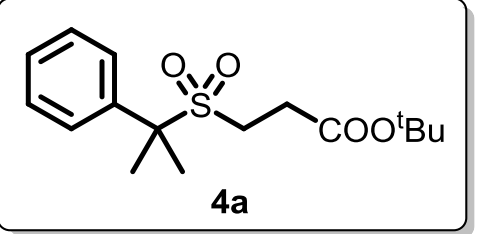

tert-butyl 3-((2-phenylpropan-2-yl) sulfonyl) propanoate (4a). Prepared following general procedure II using 1,3-dioxoisoindolin-2-yl 2methyl-2-phenylpropanoate $(61.9 \mathrm{mg}, 0.2 \mathrm{mmol})$,

$\mathrm{Na}_{2} \mathrm{~S}_{2} \mathrm{O}_{4}(52.2 \mathrm{mg}, 0.3 \mathrm{mmol})$, tert-butyl acrylate $(76.9 \mathrm{mg}, 0.6 \mathrm{mmol})$, $\mathrm{Et}_{3} \mathrm{~N} \cdot \mathrm{HCl}(68.8 \mathrm{mg}, 0.5 \mathrm{mmol})$, LiOAc $(13.2 \mathrm{mg}, 0.2 \mathrm{mmol})$ and DMF $(2 \mathrm{~mL})$, the reaction was stirred at $100{ }^{\circ} \mathrm{C}$ oil bath for $15 \mathrm{~h}$ giving $4 \mathrm{a}$ in $71 \%$ yield $(43.9$ $\mathrm{mg}$ ) as a white solid by column chromatography (PE/EA $\left.=5: 1, R_{f}=0.3\right) .{ }^{1} \mathbf{H}$ NMR $\left(400 \mathrm{MHz}, \mathrm{CDCl}_{3}\right) \delta 7.64-7.62(\mathrm{~m}, 2 \mathrm{H}), 7.42-7.36(\mathrm{~m}, 3 \mathrm{H}), 2.88(\mathrm{t}, J=$ $8.0 \mathrm{~Hz}, 2 \mathrm{H}), 2.52(\mathrm{t}, J=8.0 \mathrm{~Hz}, 2 \mathrm{H}), 1.85(\mathrm{~s}, 6 \mathrm{H}), 1.39(\mathrm{~s}, 9 \mathrm{H}) .{ }^{13} \mathrm{C}$ NMR $(100$ $\left.\mathrm{MHz}, \mathrm{CDCl}_{3}\right) \delta 169.7,137.2,128.7,128.6,128.0,81.6,64.9,42.0,27.9,26.8$, 22.4. IR $\vee 2979,2934,1731,1368,1302,1120,1098,779,700 \mathrm{~cm}^{-1}$; HRMS (ESI) $\mathrm{m} / \mathrm{z}:[\mathrm{M}+\mathrm{Na}]^{+}$Calcd. for $\mathrm{C}_{16} \mathrm{H}_{24} \mathrm{O}_{4} \mathrm{NaS} 335.1293$; found 335.1277 .

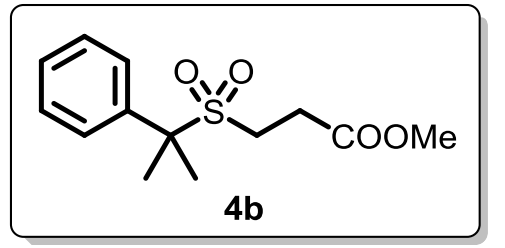

tert-butyl 3-((2-phenylpropan-2-yl) sulfonyl) propanoate $(\mathbf{4 b})$. Prepared following general procedure II using 1,3-dioxoisoindolin-2-yl 2-methyl-2-phenylpropanoate $\left(\begin{array}{llll}61.9 & \mathrm{mg}, & 0.2\end{array}\right.$ mmol), $\mathrm{Na}_{2} \mathrm{~S}_{2} \mathrm{O}_{4}(52.2 \mathrm{mg}, 0.3 \mathrm{mmol})$, methyl acrylate $(51.6 \mathrm{mg}, 0.6 \mathrm{mmol})$, $\mathrm{Et}_{3} \mathrm{~N} \cdot \mathrm{HCl}(68.8 \mathrm{mg}, 0.5 \mathrm{mmol})$, LiOAc $(13.2 \mathrm{mg}, 0.2 \mathrm{mmol})$ and DMF $(2 \mathrm{~mL})$, the reaction was stirred at $100{ }^{\circ} \mathrm{C}$ oil bath for $15 \mathrm{~h}$ giving $\mathbf{4 b}$ in $54 \%$ yield $(29.1$ $\mathrm{mg})$ as an oil by column chromatography (PE/EA $\left.=2: 1, \mathrm{R}_{\mathrm{f}}=0.3\right) .{ }^{1} \mathrm{H}$ NMR $(400$ $\left.\mathrm{MHz}, \mathrm{CDCl}_{3}\right) \delta 7.67-7.59(\mathrm{~m}, 2 \mathrm{H}), 7.45-7.35(\mathrm{~m}, 3 \mathrm{H}), 3.65(\mathrm{~s}, 3 \mathrm{H}), 2.94(\mathrm{t}, J$ $=8.0 \mathrm{~Hz}, 2 \mathrm{H}), 2.6(\mathrm{t}, J=8.0 \mathrm{~Hz}, 2 \mathrm{H}), 1.86(\mathrm{~s}, 6 \mathrm{H}) \cdot{ }^{13} \mathrm{C} \mathrm{NMR}\left(100 \mathrm{MHz}, \mathrm{CDCl}_{3}\right)$ $\delta$ 171.1, 137.0, 128.8, 128.7, 128.0, 65.0, 52.2, 42.0, 25.7, 22.4. IR $\vee 2954$, 1739, 1438, 1364, 1301, 1119, 1097, 778, $700 \mathrm{~cm}^{-1}$; HRMS (ESI) m/z: [M + $\mathrm{Na}]^{+}$Calcd. for $\mathrm{C}_{13} \mathrm{H}_{18} \mathrm{O}_{4} \mathrm{NaS} 293.0823$; found 293.0816. 


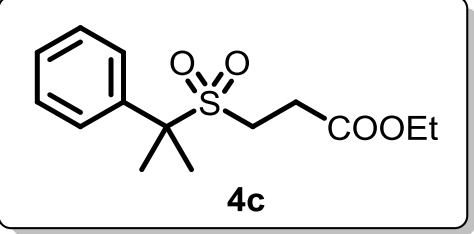

ethyl 3-((2-phenylpropan-2-yl) sulfonyl) propanoate (4c). Prepared following general procedure II using 1,3-dioxoisoindolin-2-yl 2methyl-2-phenylpropanoate $(61.9 \mathrm{mg}, 0.2 \mathrm{mmol})$, $\mathrm{Na}_{2} \mathrm{~S}_{2} \mathrm{O}_{4}(52.2 \mathrm{mg}, 0.3 \mathrm{mmol})$, ethyl acrylate $(60.1 \mathrm{mg}, 0.6 \mathrm{mmol}), \mathrm{Et}_{3} \mathrm{~N} \cdot \mathrm{HCl}$ (68.8 $\mathrm{mg}, 0.5 \mathrm{mmol})$, LiOAc (13.2 $\mathrm{mg}, 0.2 \mathrm{mmol})$ and DMF (2 mL), the reaction was stirred at $100{ }^{\circ} \mathrm{C}$ oil bath for $15 \mathrm{~h}$ giving $4 \mathrm{c}$ in $53 \%$ yield $(30.0 \mathrm{mg})$ as an oil by column chromatography $\left(\mathrm{PE} / \mathrm{EA}=2: 1, \mathrm{R}_{\mathrm{f}}=0.4\right) .{ }^{1} \mathrm{H} \mathbf{N M R}\left(400 \mathrm{MHz}, \mathrm{CDCl}_{3}\right)$ $\delta 7.68-7.60(\mathrm{~m}, 2 \mathrm{H}), 7.42-7.37(\mathrm{~m}, 3 \mathrm{H}), 4.10$ (q, J = 7.1 Hz, 2H), 2.94 (t, $J=$ $8.0 \mathrm{~Hz}, 2 \mathrm{H}), 2.58(\mathrm{t}, J=8.0 \mathrm{~Hz}, 2 \mathrm{H}), 1.86(\mathrm{~s}, 6 \mathrm{H}), 1.21(\mathrm{t}, J=7.1 \mathrm{~Hz}, 3 \mathrm{H}) .{ }^{13} \mathrm{C}$ NMR $\left(100 \mathrm{MHz}, \mathrm{CDCl}_{3}\right) \delta 170.6,137.1,128.8,128.7,128.0,65.0,61.2,42.0$, 25.9, 22.4, 14.0. IR $\vee 2985,2930,1735,1300,1189,1119,1097,777,699 \mathrm{~cm}^{-1}$ HRMS (ESI) m/z: $[\mathrm{M}+\mathrm{Na}]^{+}$Calcd. for $\mathrm{C}_{14} \mathrm{H}_{20} \mathrm{O}_{4} \mathrm{NaS}$ 307.0980; found 307.0973.

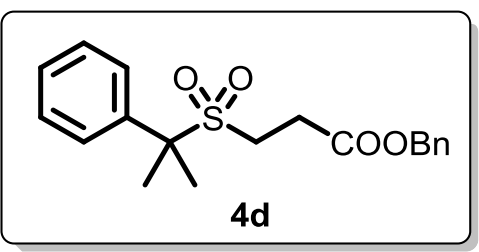

benzyl 3-((2-phenylpropan-2-yl) sulfonyl) propanoate $(\mathbf{4 d})$. Prepared following general procedure II using 1,3-dioxoisoindolin-2-yl 2methyl-2-phenylpropanoate $(61.9 \mathrm{mg}, 0.2 \mathrm{mmol})$, $\mathrm{Na}_{2} \mathrm{~S}_{2} \mathrm{O}_{4}(52.2 \mathrm{mg}, 0.3 \mathrm{mmol})$, benzyl acrylate $(97.3 \mathrm{mg}, 0.6 \mathrm{mmol}), \mathrm{Et}_{3} \mathrm{~N} \cdot \mathrm{HCl}$ (68.8 $\mathrm{mg}, 0.5 \mathrm{mmol})$, LiOAC (13.2 $\mathrm{mg}, 0.2 \mathrm{mmol}$ ) and DMF (2 mL), the reaction was stirred at $100{ }^{\circ} \mathrm{C}$ oil bath for $15 \mathrm{~h}$ giving $4 \mathrm{~d}$ in $67 \%$ yield $(46.3 \mathrm{mg})$ as a white solid by column chromatography (PE/EA $\left.=5: 1, R_{f}=0.3\right) .{ }^{1} \mathbf{H}$ NMR (400 $\left.\mathrm{MHz}, \mathrm{CDCl}_{3}\right) \delta 7.67-7.60(\mathrm{~m}, 2 \mathrm{H}), 7.43-7.28(\mathrm{~m}, 8 \mathrm{H}), 5.09(\mathrm{~s}, 2 \mathrm{H}), 2.96(\mathrm{t}, J$ $=8.0 \mathrm{~Hz}, 2 \mathrm{H}), 2.64(\mathrm{t}, J=8.0 \mathrm{~Hz}, 2 \mathrm{H}), 1.86(\mathrm{~s}, 6 \mathrm{H}) .{ }^{13} \mathrm{C} \mathrm{NMR}\left(100 \mathrm{MHz}, \mathrm{CDCl}_{3}\right)$ $\delta 170.4,137.0,135.3,128.8,128.7,128.5,128.4,128.2,128.0,67.0,65.0$, 42.0, 26.0, 22.4. IR $\vee 3035,2928,1735,1498,1353,1298,1118,1096,777$, $696 \mathrm{~cm}^{-1}$; HRMS (ESI) m/z: [M + Na] $]^{+}$Calcd. for $\mathrm{C}_{19} \mathrm{H}_{22} \mathrm{O}_{4} \mathrm{NaS} 369.1136$; found 369.1129. 


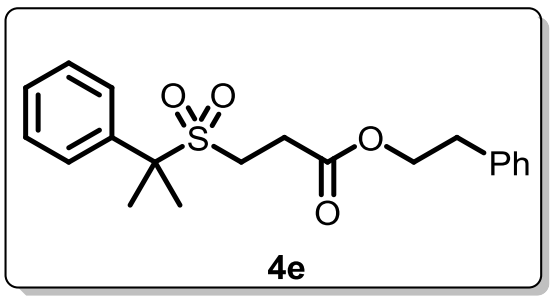

phenethyl

3-((2-phenylpropan-2-yl)

sulfonyl)propanoate

(4e). Prepared

following general procedure II using 1,3-dioxoisoindolin-2-yl 2- methyl-2- phenyl propanoate $(61.9 \mathrm{mg}, 0.2 \mathrm{mmol}), \mathrm{Na}_{2} \mathrm{~S}_{2} \mathrm{O}_{4}(52.2 \mathrm{mg}, 0.3 \mathrm{mmol})$, phenethyl acrylate (105 mg, $0.6 \mathrm{mmol}), \mathrm{Et}_{3} \mathrm{~N} \cdot \mathrm{HCl}(68.8 \mathrm{mg}, 0.5 \mathrm{mmol}), \mathrm{LiOAc}(13.2 \mathrm{mg}$, $0.2 \mathrm{mmol}$ ) and DMF $(2 \mathrm{~mL})$, the reaction was stirred at $100{ }^{\circ} \mathrm{C}$ oil bath for $15 \mathrm{~h}$ giving $4 \mathrm{e}$ in $58 \%$ yield $(42.0 \mathrm{mg})$ as an oil by column chromatography $(\mathrm{PE} / \mathrm{EA}=$ $\left.5: 1, \mathrm{R}_{\mathrm{f}}=0.3\right) .{ }^{1} \mathrm{H}$ NMR $\left(400 \mathrm{MHz}, \mathrm{CDCl}_{3}\right) \delta 7.63-7.60(\mathrm{~m}, 2 \mathrm{H}), 7.44-7.33(\mathrm{~m}$, 3H), $7.32-7.20(\mathrm{~m}, 3 \mathrm{H}), 7.17-7.15(\mathrm{~m}, 2 \mathrm{H}), 4.25(\mathrm{t}, J=7.0 \mathrm{~Hz}, 2 \mathrm{H}), 2.92-$ $2.84(\mathrm{~m}, 4 \mathrm{H}), 2.60-2.50(\mathrm{~m}, 2 \mathrm{H}), 1.84(\mathrm{~s}, 6 \mathrm{H}) .{ }^{13} \mathrm{C}$ NMR $\left(100 \mathrm{MHz}, \mathrm{CDCl}_{3}\right) \delta$ $170.5,137.4,137.0,128.8(0), 128.7(5), 128.6(5), 128.5,128.0,126.6,65.59$, 65.0, 41.9, 34.9, 25.9, 22.4. IR $\vee 2958,1735,1498,1356,1299,1119,1097$, $777,698 \mathrm{~cm}^{-1}$; HRMS (ESI) m/z: [M + Na] $]^{+}$Calcd. for $\mathrm{C}_{20} \mathrm{H}_{24} \mathrm{O}_{4} \mathrm{NaS} 383.1293$; found 383.1283 .

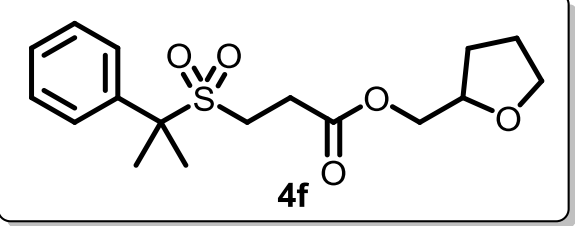

(tetrahydrofuran-2-yl)methyl 3-((2-phenyl propan-2-yl)sulfonyl)propanoate (4f). Prepared following general procedure II using 1,3-dioxoisoindolin-2-yl 2- methyl-2phenyl propanoate $(61.9 \mathrm{mg}, 0.2 \mathrm{mmol}), \mathrm{Na}_{2} \mathrm{~S}_{2} \mathrm{O}_{4}(52.2 \mathrm{mg}, 0.3 \mathrm{mmol})$, (tetrahydrofuran-2-yl)methyl acrylate $(93.6 \mathrm{mg}, 0.6 \mathrm{mmol}), \mathrm{Et}_{3} \mathrm{~N} \cdot \mathrm{HCl}(68.8 \mathrm{mg}$, $0.5 \mathrm{mmol})$, LiOAc (13.2 $\mathrm{mg}, 0.2 \mathrm{mmol})$ and DMF (2 mL), the reaction was stirred at $100{ }^{\circ} \mathrm{C}$ oil bath for $15 \mathrm{~h}$ giving $4 \mathrm{f}$ in $59 \%$ yield $(39.9 \mathrm{mg})$ as an oil by column chromatography (PE/EA $\left.=5: 1, \mathrm{R}_{\mathrm{f}}=0.3\right) .{ }^{1} \mathrm{H}$ NMR $\left(400 \mathrm{MHz}, \mathrm{CDCl}_{3}\right) \delta$ $7.67-7.58(\mathrm{~m}, 2 \mathrm{H}), 7.44-7.31(\mathrm{~m}, 3 \mathrm{H}), 4.13-3.96(\mathrm{~m}, 3 \mathrm{H}), 3.86-3.73(\mathrm{~m}$, 2H), $2.93(\mathrm{t}, J=8.0 \mathrm{~Hz}, 2 \mathrm{H}), 2.63(\mathrm{t}, J=8.0 \mathrm{~Hz}, 2 \mathrm{H}), 2.01-1.87(\mathrm{~m}, 3 \mathrm{H}), 1.85$ $(\mathrm{s}, 6 \mathrm{H}), 1.60-1.53(\mathrm{~m}, 1 \mathrm{H}) .{ }^{13} \mathrm{C} \mathrm{NMR}\left(100 \mathrm{MHz}, \mathrm{CDCl}_{3}\right) \delta 170.6,137.0,128.8$, $128.7,128.0,76.2,68.4,67.1,65.0,42.0,27.9,25.9,25.6,22.4$. IR $\vee 2948$, 
2871, 1735, 1391, 1300, 1160, 1119, 1097, 778, $700 \mathrm{~cm}^{-1}$; HRMS (ESI) m/z: [M $+\mathrm{H}]^{+}$Calcd. for $\mathrm{C}_{17} \mathrm{H}_{25} \mathrm{O}_{5} \mathrm{~S} 341.1423$; found 341.1414.

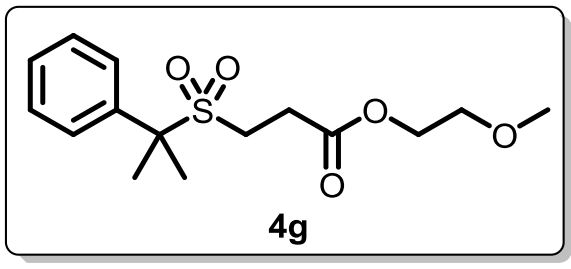

2-methoxyethyl 3-((2-phenylpropan-2-yl) sulfonyl)propanoate $(\mathbf{4 g})$. Prepared following general procedure II using 1,3-dioxoisoindolin2-yl 2- methyl-2- phenyl propanoate $(61.9 \mathrm{mg}$, $0.2 \mathrm{mmol}), \mathrm{Na}_{2} \mathrm{~S}_{2} \mathrm{O}_{4}(52.2 \mathrm{mg}, 0.3 \mathrm{mmol}), 2$-methoxyethyl acrylate $(81.6 \mathrm{mg}$, $0.6 \mathrm{mmol}), \mathrm{Et}_{3} \mathrm{~N} \cdot \mathrm{HCl}(68.8 \mathrm{mg}, 0.5 \mathrm{mmol}), \mathrm{LiOAc}(13.2 \mathrm{mg}, 0.2 \mathrm{mmol})$ and DMF ( $2 \mathrm{~mL}$ ), the reaction was stirred at $100^{\circ} \mathrm{C}$ oil bath for $15 \mathrm{~h}$ giving $\mathbf{4 g}$ in $71 \%$ yield (44.4 mg) as an oil by column chromatography (PE/EA $\left.=2: 1, R_{f}=0.4\right) .{ }^{1} \mathrm{H}$ NMR $\left(400 \mathrm{MHz}, \mathrm{CDCl}_{3}\right) \delta 7.65-7.60(\mathrm{~m}, 2 \mathrm{H}), 7.43-7.34(\mathrm{~m}, 3 \mathrm{H}), 4.22-4.17$ (m, 2H), $3.56-3.51(\mathrm{~m}, 2 \mathrm{H}), 3.34(\mathrm{~s}, 3 \mathrm{H}), 2.96-2.92(\mathrm{~m}, 2 \mathrm{H}), 2.65-2.61(\mathrm{~m}$, $2 \mathrm{H}), 1.85(\mathrm{~s}, 6 \mathrm{H}) .{ }^{13} \mathrm{C}$ NMR $\left(100 \mathrm{MHz}, \mathrm{CDCl}_{3}\right) \delta 170.6,137.0,128.7,128.6$, 128.0, 70.1, 65.0, 64.2, 58.9, 42.0, 25.9, 22.4.IR $\vee 2926,1735,1378,1298$, 1160, 1118, 1096, 776, $698 \mathrm{~cm}^{-1}$; HRMS (ESI) m/z: $[\mathrm{M}+\mathrm{Na}]^{+}$Calcd. for $\mathrm{C}_{15} \mathrm{H}_{22} \mathrm{O}_{5} \mathrm{NaS} 337.1086$; found 337.1073.

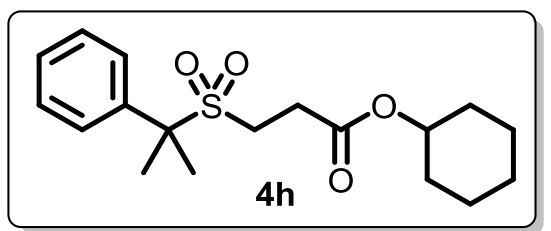

cyclohexyl

3-((2-phenylpropan-2-yl) sulfonyl)propanoate $(4 \mathrm{~h})$. Prepared following general procedure II using 1,3-dioxoisoindolin2-yl 2- methyl-2- phenyl propanoate $(61.9 \mathrm{mg}$, $0.2 \mathrm{mmol}), \mathrm{Na}_{2} \mathrm{~S}_{2} \mathrm{O}_{4}(52.2 \mathrm{mg}, 0.3 \mathrm{mmol})$, cyclohexyl acrylate $(92.5 \mathrm{mg}, 0.6$ $\mathrm{mmol}), \mathrm{Et}_{3} \mathrm{~N} \cdot \mathrm{HCl}(68.8 \mathrm{mg}, 0.5 \mathrm{mmol}), \mathrm{LiOAc}(13.2 \mathrm{mg}, 0.2 \mathrm{mmol})$ and DMF (2 $\mathrm{mL}$ ), the reaction was stirred at $100{ }^{\circ} \mathrm{C}$ oil bath for $15 \mathrm{~h}$ giving $4 \mathrm{~h}$ in $74 \%$ yield $(50.0 \mathrm{mg})$ as an oil by column chromatography (PE/EA $\left.=5: 1, \mathrm{R}_{\mathrm{f}}=0.3\right) .{ }^{1} \mathrm{H}$ NMR $\left(400 \mathrm{MHz}, \mathrm{CDCl}_{3}\right) \delta 7.70-7.59(\mathrm{~m}, 2 \mathrm{H}), 7.43-7.36(\mathrm{~m}, 3 \mathrm{H}), 4.76-4.66$ $(\mathrm{m}, 1 \mathrm{H}), 2.97-2.88(\mathrm{~m}, 2 \mathrm{H}), 2.62-2.52(\mathrm{~m}, 2 \mathrm{H}), 1.86(\mathrm{~s}, 6 \mathrm{H}), 1.80-1.73(\mathrm{~m}$, 2H), $1.70-1.64(\mathrm{~m}, 2 \mathrm{H}), 1.55-1.47(\mathrm{~m}, 1 \mathrm{H}), 1.40-1.24(\mathrm{~m}, 5 \mathrm{H}) .{ }^{13} \mathbf{C}$ NMR $\left(100 \mathrm{MHz}, \mathrm{CDCl}_{3}\right) \delta 170.0,137.1,128.7,128.6,128.0,73.6,65.0,42.0,31.4$, 
26.2, 25.2, 23.5, 22.4.IR $\vee 2938,2860,1731,1450,1360,1301,1120,1098$, 777, $699 \mathrm{~cm}^{-1}$; HRMS (ESI) m/z: [M + Na] $]^{+}$Calcd. for $\mathrm{C}_{18} \mathrm{H}_{26} \mathrm{O}_{4} \mathrm{NaS} 361.1449$; found 361.1439 .

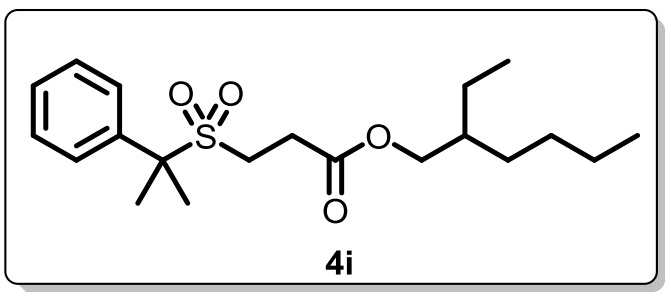

\section{2-ethylhexyl 3-((2-phenylpropan-2-yl)} sulfonyl)propanoate (4i). Prepared following general procedure II using 1,3-dioxoisoindolin- 2-yl 2- methyl-2phenyl propanoate $(61.9 \mathrm{mg}, 0.2 \mathrm{mmol}), \mathrm{Na}_{2} \mathrm{~S}_{2} \mathrm{O}_{4}(52.2 \mathrm{mg}, 0.3 \mathrm{mmol})$, 2-ethylhexyl acrylate $(110.5 \mathrm{mg}, 0.6 \mathrm{mmol}), \mathrm{Et}_{3} \mathrm{~N} \cdot \mathrm{HCl}(68.8 \mathrm{mg}, 0.5 \mathrm{mmol})$, LiOAc (13.2 mg, $0.2 \mathrm{mmol})$ and DMF $(2 \mathrm{~mL})$, the reaction was stirred at $100{ }^{\circ} \mathrm{C}$ oil bath for $15 \mathrm{~h}$ giving $4 \mathbf{i}$ in $75 \%$ yield $(55.0 \mathrm{mg})$ as an oil by column chromatography $\left(\mathrm{PE} / \mathrm{EA}=5: 1, \mathrm{R}_{\mathrm{f}}=0.4\right) .{ }^{1} \mathrm{H}$ NMR $\left(400 \mathrm{MHz}, \mathrm{CDCl}_{3}\right) \delta 7.65-$ $7.62(\mathrm{~m}, 2 \mathrm{H}), 7.45-7.35(\mathrm{~m}, 3 \mathrm{H}), 3.96-3.94(\mathrm{~m}, 2 \mathrm{H}), 2.94-2.90(\mathrm{~m}, 2 \mathrm{H})$, $2.61-2.57(\mathrm{~m}, 2 \mathrm{H}), 1.86(\mathrm{~s}, 6 \mathrm{H}), 1.54-1.48(\mathrm{~m}, 1 \mathrm{H}), 1.29-1.21(\mathrm{~m}, 8 \mathrm{H})$, $0.90-0.83(\mathrm{~m}, 6 \mathrm{H}) .{ }^{13} \mathrm{C}$ NMR $\left(100 \mathrm{MHz}, \mathrm{CDCl}_{3}\right) \delta 170.7,137.1,128.7(3)$, 128.6(5), 128.0, 67.6, 65.0, 42.0, 38.6, 30.3, 28.8, 25.9, 23.6, 22.9, 22.4, 14.0, 10.9. IR $\vee 2958,2930,1735,1464,1302,1245,1183,1120,1098,777,698$ $\mathrm{cm}^{-1}$; HRMS (ESI) m/z: $[\mathrm{M}+\mathrm{Na}]^{+}$Calcd. for $\mathrm{C}_{20} \mathrm{H}_{32} \mathrm{O}_{4} \mathrm{NaS}$ 391.1919; found 391.1905.

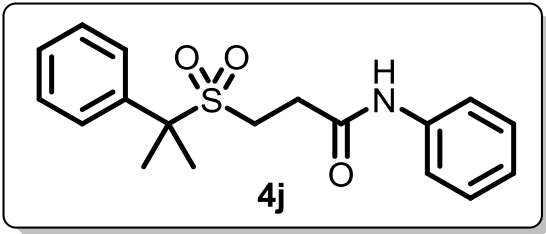

N-phenyl-3-((2-phenylpropan-2-yl) sulfonyl)propanamide (4j). Prepared following general procedure II using 1,3-dioxoisoindolin- 2-yl 2- methyl-2- phenyl propanoate $(61.9 \mathrm{mg}, \quad 0.2 \mathrm{mmol}), \mathrm{Na}_{2} \mathrm{~S}_{2} \mathrm{O}_{4} \quad(52.2 \mathrm{mg}, 0.3 \mathrm{mmol})$, $\mathrm{N}$-phenylacrylamide $(88.3 \mathrm{mg}, 0.6 \mathrm{mmol}), \mathrm{Et}_{3} \mathrm{~N} \cdot \mathrm{HCl}(68.8 \mathrm{mg}, 0.5 \mathrm{mmol})$, $\mathrm{LiOAC}(13.2 \mathrm{mg}, 0.2 \mathrm{mmol})$ and DMF $(2 \mathrm{~mL})$, the reaction was stirred at $100{ }^{\circ} \mathrm{C}$ oil bath for $15 \mathrm{~h}$ giving $4 \mathrm{j}$ in $51 \%$ yield $(33.9 \mathrm{mg}$ ) as a yellow solid by column chromatography $\left(\mathrm{PE} / \mathrm{EA}=1: 1, \mathrm{R}_{\mathrm{f}}=0.2\right) .{ }^{1} \mathrm{H} \mathrm{NMR}\left(400 \mathrm{MHz}, \mathrm{CDCl}_{3}\right) \delta 8.11(\mathrm{~s}$, 
1H), $7.63-7.60(m, 2 H), 7.43-7.34(m, 5 H), 7.28-7.24(m, 2 H), 7.08-7.05$ $(\mathrm{m}, 1 \mathrm{H}), 3.12-3.02(\mathrm{~m}, 2 \mathrm{H}), 2.77-2.68(\mathrm{~m}, 2 \mathrm{H}), 1.87(\mathrm{~s}, 6 \mathrm{H}) .{ }^{13} \mathrm{C}$ NMR $(100$ $\left.\mathrm{MHz}, \mathrm{CDCl}_{3}\right) \delta 167.7,137.8,136.7,128.9,128.8,128.1,124.3,119.9,65.2$, 42.2, 28.0, 22.5.IR v 3342, 1692, 1601, 1544, 1499, 1443, 1273, 1118, 1096, 757, $695 \mathrm{~cm}^{-1}$; HRMS (ESI) m/z: $[\mathrm{M}+\mathrm{H}]^{+}$Calcd. for $\mathrm{C}_{18} \mathrm{H}_{22} \mathrm{NO}_{3} \mathrm{~S} 332.1320$; found 332.1310 .

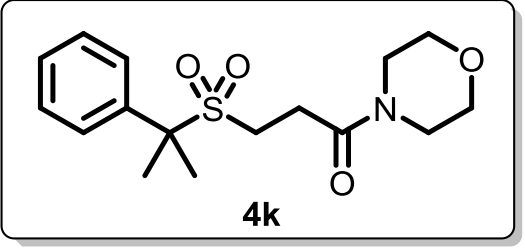

1-morpholino-3-((2-phenylpropan-2-yl)sulfo nyl)propan-1-one (4k). Prepared following general procedure II using 1,3-dioxoisoindolin2-yl 2- methyl-2- phenyl propanoate $(61.9 \mathrm{mg}$, $0.2 \mathrm{mmol}$ ), $\mathrm{Na}_{2} \mathrm{~S}_{2} \mathrm{O}_{4}$ (52.2 mg, $0.3 \mathrm{mmol}$ ), 1-morpholinoprop-2-en-1-one (84.7 $\mathrm{mg}, 0.6 \mathrm{mmol}), \mathrm{Et}_{3} \mathrm{~N} \cdot \mathrm{HCl}(68.8 \mathrm{mg}, 0.5 \mathrm{mmol}), \mathrm{LiOAC}(13.2 \mathrm{mg}, 0.2 \mathrm{mmol})$ and DMF $(2 \mathrm{~mL})$, the reaction was stirred at $100{ }^{\circ} \mathrm{C}$ oil bath for $15 \mathrm{~h}$ giving $4 \mathbf{k}$ in $62 \%$ yield $(40.2 \mathrm{mg})$ as a white solid by column chromatography $\left(\mathrm{PE} / \mathrm{EA}=1: 1, \mathrm{R}_{\mathrm{f}}=\right.$ 0.2). ${ }^{1} \mathrm{H}$ NMR (400 MHz, $\left.\mathrm{CDCl}_{3}\right) \delta 7.68-7.58(\mathrm{~m}, 2 \mathrm{H}), 7.43-7.32(\mathrm{~m}, 3 \mathrm{H})$, $3.62-3.57(\mathrm{~m}, 4 \mathrm{H}), 3.56-3.49(\mathrm{~m}, 2 \mathrm{H}), 3.39-3.29(\mathrm{~m}, 2 \mathrm{H}), 3.03-2.99(\mathrm{~m}$, $2 \mathrm{H}), 2.57-2.53(\mathrm{~m}, 2 \mathrm{H}), 1.86(\mathrm{~s}, 6 \mathrm{H}) \cdot{ }^{13} \mathrm{C}$ NMR $\left(100 \mathrm{MHz}, \mathrm{CDCl}_{3}\right) \delta 167.8$, $137.1,128.6(4), 128.5(9), 128.1,66.6,66.3,64.9,45.6,42.3,42.1,24.5$, 22.3.IR $\vee 2856,1648,1443,1303,1273,1245,1118,1098,1028,779,701$ $\mathrm{cm}^{-1}$; HRMS (ESI) m/z: $[\mathrm{M}+\mathrm{H}]^{+}$Calcd. for $\mathrm{C}_{16} \mathrm{H}_{24} \mathrm{NO}_{4} \mathrm{~S}$ 326.1426; found 326.1417.

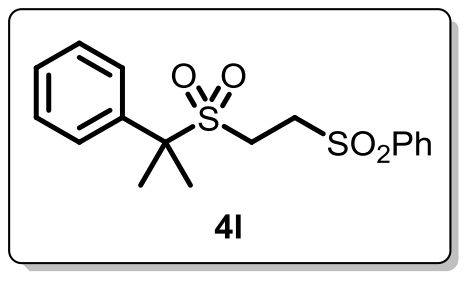

((2-((2-phenylpropan-2-yl)sulfonyl)ethyl)sulfony I)benzene (4I). Prepared following general procedure II using 1,3-dioxoisoindolin- 2-yl 2methyl-2- phenyl propanoate $(61.9 \mathrm{mg}, 0.2 \mathrm{mmol})$,

$\mathrm{Na}_{2} \mathrm{~S}_{2} \mathrm{O}_{4}(52.2 \mathrm{mg}, 0.3 \mathrm{mmol})$, (vinylsulfonyl)benzene (100.9 mg, $0.6 \mathrm{mmol}$ ), $\mathrm{Et}_{3} \mathrm{~N} \cdot \mathrm{HCl}(68.8 \mathrm{mg}, 0.5 \mathrm{mmol})$, LiOAc (13.2 mg, $\left.0.2 \mathrm{mmol}\right)$ and DMF $(2 \mathrm{~mL})$, the reaction was stirred at $100{ }^{\circ} \mathrm{C}$ oil bath for $15 \mathrm{~h}$ giving $4 \mathrm{I}$ in $61 \%$ yield $(42.9$ 
$\mathrm{mg})$ as a yellow solid by column chromatography $\left(\mathrm{PE} / \mathrm{EA}=2: 1, \mathrm{R}_{\mathrm{f}}=0.3\right) .{ }^{1} \mathrm{H}$ NMR $\left(400 \mathrm{MHz} \mathrm{CDCl}_{3}\right) \delta 7.80-7.73(\mathrm{~m}, 2 \mathrm{H}), 7.70-7.62(\mathrm{~m}, 1 \mathrm{H}), 7.59-7.52$ $(\mathrm{m}, 4 \mathrm{H}), 7.43-7.36(\mathrm{~m}, 3 \mathrm{H}), 3.10-3.01(\mathrm{~m}, 4 \mathrm{H}), 1.85(\mathrm{~s}, 6 \mathrm{H}) .{ }^{13} \mathrm{C}$ NMR $(100$ $\left.\mathrm{MHz}, \mathrm{CDCl}_{3}\right) \delta 138.2,136.3,134.3,129.5,129.1,128.9,127.9(4), 127.8(8)$, 66.0, 48.6, 40.2, 22.3.IR $\vee 3065,3002,2939,1712,1448,1300,1151,1121$, 1087, 779, 741, $700 \mathrm{~cm}^{-1}$; HRMS (ESI) m/z: $[\mathrm{M}+\mathrm{Na}]^{+}$Calcd. for $\mathrm{C}_{17} \mathrm{H}_{20} \mathrm{O}_{4} \mathrm{NaS}_{2} 375.0701$; found 375.0691.

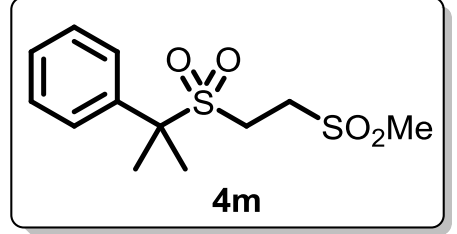

\section{(2-((2-(methylsulfonyl)ethyl)sulfonyl)propan-2-y} I)benzene $(\mathbf{4 m})$. Prepared following general procedure II using 1,3-dioxoisoindolin- 2-yl 2methyl-2- phenyl propanoate $(61.9 \mathrm{mg}, 0.2 \mathrm{mmol})$, $\mathrm{Na}_{2} \mathrm{~S}_{2} \mathrm{O}_{4}(52.2 \mathrm{mg}, 0.3 \mathrm{mmol})$, (methylsulfonyl)ethene $(63.7 \mathrm{mg}, 0.6 \mathrm{mmol})$, $\mathrm{Et}_{3} \mathrm{~N} \cdot \mathrm{HCl}(68.8 \mathrm{mg}, 0.5 \mathrm{mmol})$, LiOAc (13.2 mg, $\left.0.2 \mathrm{mmol}\right)$ and DMF (2 mL), the reaction was stirred at $100{ }^{\circ} \mathrm{C}$ oil bath for $15 \mathrm{~h}$ giving $4 \mathrm{~m}$ in $59 \%$ yield $(34.2$ $\mathrm{mg}$ ) as a white solid by column chromatography (PE/EA $\left.=2: 1, \mathrm{R}_{\mathrm{f}}=0.3\right) .{ }^{1} \mathrm{H}$ NMR $\left(400 \mathrm{MHz} \mathrm{CDCl}_{3}\right) \delta 7.64-7.61(\mathrm{~m}, 2 \mathrm{H}), 7.48$ - $7.35(\mathrm{~m}, 3 \mathrm{H}), 3.16-3.07$ $(\mathrm{m}, 4 \mathrm{H}), 2.86(\mathrm{~s}, 3 \mathrm{H}), 1.88(\mathrm{~s}, 6 \mathrm{H}) .{ }^{13} \mathrm{C}$ NMR $\left(100 \mathrm{MHz}, \mathrm{CDCl}_{3}\right) \delta$ 136.3, 129.2, 128.9, 128.0, 65.9, 46.8, 41.4, 39.6, 22.3.IR 3013, 2986, 2931, 1711, 1369, 1286, 1140, 1115, 793, 780, $697 \mathrm{~cm}^{-1}$; HRMS (ESI) m/z: [M + Na] $]^{+}$Calcd. for $\mathrm{C}_{12} \mathrm{H}_{18} \mathrm{O}_{4} \mathrm{NaS}_{2}$ 313.0544; found 313.0532.

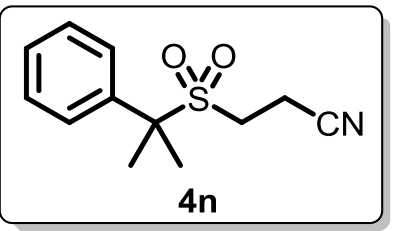

\section{3-((2-phenylpropan-2-yl)sulfonyl) propanenitrile} (14). Prepared following general procedure II using 1,3-dioxoisoindolin- 2-yl 2- methyl-2- phenyl propanoate $(61.9 \mathrm{mg}, 0.2 \mathrm{mmol}), \mathrm{Na}_{2} \mathrm{~S}_{2} \mathrm{O}_{4}(52.2 \mathrm{mg}$, $0.3 \mathrm{mmol}$ ), acrylonitrile (31.8 mg, $0.6 \mathrm{mmol}), \mathrm{Et}_{3} \mathrm{~N} \cdot \mathrm{HCl}(68.8 \mathrm{mg}, 0.5 \mathrm{mmol})$, LiOAc (13.2 mg, $0.2 \mathrm{mmol})$ and DMF $(2 \mathrm{~mL})$, the reaction was stirred at $100{ }^{\circ} \mathrm{C}$ for $15 \mathrm{~h}$ giving 14 in $53 \%$ yield $(25.0 \mathrm{mg}$ ) as an oil by column chromatography $\left(\mathrm{PE} / \mathrm{EA}=2: 1, \mathrm{R}_{\mathrm{f}}=0.3\right) .{ }^{1} \mathrm{H}$ NMR $\left(400 \mathrm{MHz} \mathrm{CDCl}_{3}\right) \delta 7.69-7.55(\mathrm{~m}, 2 \mathrm{H}), 7.52$ 
- $7.32(\mathrm{~m}, 3 \mathrm{H}), 2.96-2.85(\mathrm{~m}, 2 \mathrm{H}), 2.58-2.47(\mathrm{~m}, 2 \mathrm{H}), 1.88(\mathrm{~s}, 6 \mathrm{H}) .{ }^{13} \mathrm{C}$ NMR $\left(100 \mathrm{MHz}, \mathrm{CDCl}_{3}\right) \delta 136.4,129.3,129.0,127.9,116.6,65.6,42.2,22.2$, 10.5.IR v 2920, 2850, 2253, 1713, 1291, 1159, 1118, 1096, 776, $698 \mathrm{~cm}^{-1}$; HRMS (ESI) $\mathrm{m} / \mathrm{z}:[\mathrm{M}+\mathrm{Na}]^{+}$Calcd. for $\mathrm{C}_{12} \mathrm{H}_{15} \mathrm{NO}_{2} \mathrm{NaS} 260.0721$; found 260.0715 .

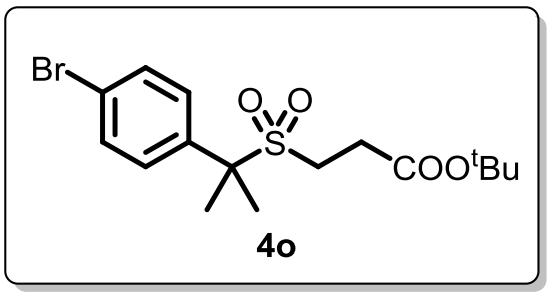

tert-butyl 3-((2-(4-bromophenyl)propan -2-yl)sulfonyl)propanoate (40). Prepared following general procedure II using 1,3-dioxoisoindolin-2-yl 2-(4-bromophenyl)-2methylpropanoate $(77.6 \mathrm{mg}, 0.2 \mathrm{mmol}), \mathrm{Na}_{2} \mathrm{~S}_{2} \mathrm{O}_{4}(52.2 \mathrm{mg}, 0.3 \mathrm{mmol}$ ), tert-Butyl acrylate $(76.9 \mathrm{mg}, 0.6 \mathrm{mmol}), \mathrm{Et}_{3} \mathrm{~N} \cdot \mathrm{HCl}(68.8 \mathrm{mg}, 0.5 \mathrm{mmol}), \mathrm{LiOAC}$ (13.2 $\mathrm{mg}, 0.2 \mathrm{mmol})$ and DMF $(2 \mathrm{~mL})$, the reaction was stirred at $100{ }^{\circ} \mathrm{C}$ oil bath for $15 \mathrm{~h}$ giving 40 in $60 \%$ yield $(46.6 \mathrm{mg}$ ) as a white solid by column chromatography $\left(\mathrm{PE} / \mathrm{EA}=5: 1, \mathrm{R}_{\mathrm{f}}=0.3\right) .{ }^{1} \mathrm{H}$ NMR $\left(400 \mathrm{MHz}, \mathrm{CDCl}_{3}\right) \delta 7.56-$ $7.45(\mathrm{~m}, 4 \mathrm{H}), 2.94-2.86(\mathrm{~m}, 2 \mathrm{H}), 2.60-2.56(\mathrm{~m}, 2 \mathrm{H}), 1.83(\mathrm{~s}, 6 \mathrm{H}), 1.41$ (s, 9H). ${ }^{13} \mathrm{C}$ NMR $\left(100 \mathrm{MHz}, \mathrm{CDCl}_{3}\right) \delta 169.7,136.3,131.8,129.8,123.3,81.8$, 64.6, 42.0, 27.9, 26.7, 22.4.IR $\vee 2979,2930,1730,1368,1302,1155,1120$, 1096, 1010, 830, $711 \mathrm{~cm}^{-1}$; HRMS (ESI) m/z: $\left[\mathrm{M}+\mathrm{Na}^{+}\right.$Calcd. for $\mathrm{C}_{16} \mathrm{H}_{23} \mathrm{BrO}_{4} \mathrm{NaS}$ 413.0398, 415.0378; found 413.0384, 415.0363.

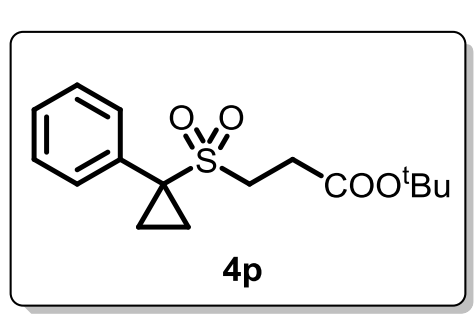

tert-butyl 3-((1-phenylcyclopropyl)sulfonyl) propanoate $(4 p)$. Prepared following general procedure II using 1,3 -dioxoisoindolin-2-yl 1-phenylcyclopropane-1-carboxylate $(61.5 \mathrm{mg}, 0.2$ $\mathrm{mmol}), \mathrm{Na}_{2} \mathrm{~S}_{2} \mathrm{O}_{4}(52.2 \mathrm{mg}, 0.3 \mathrm{mmol})$, tert-Butyl acrylate $(76.9 \mathrm{mg}, 0.6 \mathrm{mmol}), \mathrm{Et}_{3} \mathrm{~N} \cdot \mathrm{HCl}(68.8 \mathrm{mg}, 0.5 \mathrm{mmol}), \mathrm{LiOAc}(13.2 \mathrm{mg}$, $0.2 \mathrm{mmol})$ and DMF $(2 \mathrm{~mL})$, the reaction was stirred at $100^{\circ} \mathrm{C}$ oil bath for $15 \mathrm{~h}$ giving $4 p$ in $50 \%$ yield $(31 \mathrm{mg}$ ) as a white solid by column chromatography $\left(\mathrm{PE} / \mathrm{EA}=5: 1, \mathrm{R}_{\mathrm{f}}=0.3\right) .{ }^{1} \mathrm{H}$ NMR $\left(400 \mathrm{MHz}, \mathrm{CDCl}_{3}\right) \delta 7.59-7.56(\mathrm{~m}, 2 \mathrm{H}), 7.40$ 
$-7.36(\mathrm{~m}, 3 \mathrm{H}), 3.18-3.14(\mathrm{~m}, 2 \mathrm{H}), 2.67-2.63(\mathrm{~m}, 2 \mathrm{H}), 1.82(\mathrm{q}, J=4.5 \mathrm{~Hz}$, 2H), $1.42(\mathrm{~s}, 9 \mathrm{H}), 1.30-1.27(\mathrm{~m}, 2 \mathrm{H}) \cdot{ }^{13} \mathrm{C}$ NMR $\left(100 \mathrm{MHz}, \mathrm{CDCl}_{3}\right) \delta$ 169.6, 134.1, 131.8, 129.2, 128.9, 81.8, 45.6, 45.0, 28.0, 27.7, 11.9.IR $\vee 2978,2931$, 1727, 1368, 1307, 1252, 1153, 1131, 805, $700 \mathrm{~cm}^{-1}$; HRMS (ESI) m/z: [M + $\mathrm{Na}]^{+}$Calcd. for $\mathrm{C}_{16} \mathrm{H}_{22} \mathrm{O}_{4} \mathrm{NaS} 333.1131$; found 333.1125 .

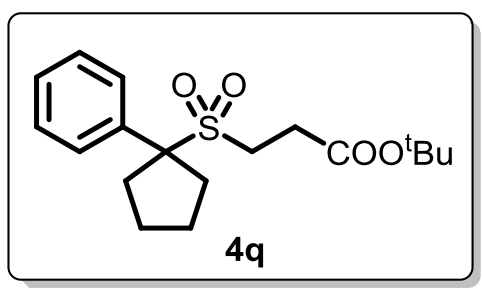

tert-butyl 3-((1-phenylcyclopentyl)sulfonyl) propanoate (4q). Prepared following general procedure II using 1,3-dioxoisoindolin-2-yl 1-phenylcyclopentane -1 - carboxylate $(67.1 \mathrm{mg}$, $0.2 \mathrm{mmol}), \mathrm{Na}_{2} \mathrm{~S}_{2} \mathrm{O}_{4}(52.2 \mathrm{mg}, 0.3 \mathrm{mmol})$, tert-Butyl acrylate $(76.9 \mathrm{mg}, 0.6$ $\mathrm{mmol}$ ), $\mathrm{Et}_{3} \mathrm{~N} \cdot \mathrm{HCl}(68.8 \mathrm{mg}, 0.5 \mathrm{mmol}), \mathrm{LiOAc}(13.2 \mathrm{mg}, 0.2 \mathrm{mmol}$ ) and DMF (2 $\mathrm{mL}$ ), the reaction was stirred at $100{ }^{\circ} \mathrm{C}$ oil bath for $15 \mathrm{~h}$ giving $4 \mathrm{q}$ in $52 \%$ yield $(34.9 \mathrm{mg})$ as a yellow solid by column chromatography ( $\left.P E / E A=5: 1, R_{f}=0.3\right)$. ${ }^{1} \mathrm{H}$ NMR $\left(400 \mathrm{MHz}, \mathrm{CDCl}_{3}\right) \delta 7.60-7.57(\mathrm{~m}, 2 \mathrm{H}), 7.43-7.30(\mathrm{~m}, 3 \mathrm{H}), 2.89-$ $2.85(\mathrm{~m}, 2 \mathrm{H}), 2.80-2.70(\mathrm{~m}, 2 \mathrm{H}), 2.50-2.46(\mathrm{~m}, 2 \mathrm{H}), 2.44-2.38(\mathrm{~m}, 2 \mathrm{H})$, $2.05-1.92(\mathrm{~m}, 2 \mathrm{H}), 1.73-1.63(\mathrm{~m}, 2 \mathrm{H}), 1.39(\mathrm{~s}, 9 \mathrm{H}) .{ }^{13} \mathrm{C}$ NMR $(100 \mathrm{MHz}$, $\left.\mathrm{CDCl}_{3}\right) \delta 169.8,137.2,129.1,128.6,81.5,76.2,43.4,33.8,27.9,26.9,24.6 . I R$ v 2978, 2877, 1728, 1367, 1298, 1250, 1153, 1119, 846, $700 \mathrm{~cm}^{-1}$; HRMS (ESI) $\mathrm{m} / \mathrm{z}:[\mathrm{M}+\mathrm{Na}]^{+}$Calcd. for $\mathrm{C}_{18} \mathrm{H}_{26} \mathrm{O}_{4} \mathrm{NaS} 361.1449$; found 361.1438 .

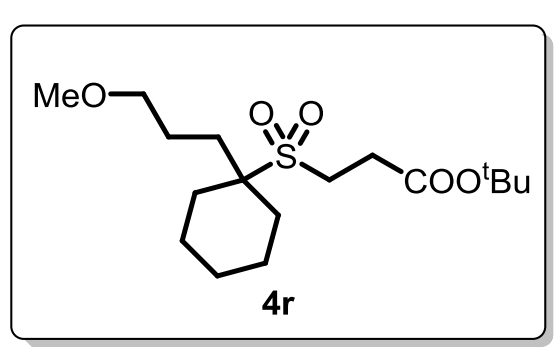

tert-butyl 3-((1-(3-methoxypropyl) cyclohexyl)sulfonyl)propanoate $(4 r)$. Prepared following general procedure II using 1,3-dioxoisoindolin-2-yl 1 - (3 - methoxypropyl) cyclohexane - 1-carboxylate $(69.0 \mathrm{mg}, 0.2$ mmol), $\mathrm{Na}_{2} \mathrm{~S}_{2} \mathrm{O}_{4}(52.2 \mathrm{mg}, 0.3 \mathrm{mmol})$, tert-Butyl acrylate $(76.9 \mathrm{mg}, 0.6 \mathrm{mmol})$, $\mathrm{Et}_{3} \mathrm{~N} \cdot \mathrm{HCl}(68.8 \mathrm{mg}, 0.5 \mathrm{mmol}), \mathrm{LiOAc}(13.2 \mathrm{mg}, 0.2 \mathrm{mmol})$ and DMF (2 mL), the reaction was stirred at $100{ }^{\circ} \mathrm{C}$ oil bath for $15 \mathrm{~h}$ giving $4 \mathrm{r}$ in $70 \%$ yield $(49.0$ $\mathrm{mg})$ as an oil by column chromatography $\left(\mathrm{PE} / \mathrm{EA}=5: 1, \mathrm{R}_{\mathrm{f}}=0.3\right) .{ }^{1} \mathrm{H}$ NMR $(400$ 
$\left.\mathrm{MHz}, \mathrm{CDCl}_{3}\right) \delta 3.39(\mathrm{t}, J=5.8 \mathrm{~Hz}, 2 \mathrm{H}), 3.32(\mathrm{~s}, 3 \mathrm{H}), 3.23-3.13(\mathrm{~m}, 2 \mathrm{H}), 2.81$ - $2.76(\mathrm{~m}, 2 \mathrm{H}), 2.00-1.91(\mathrm{~m}, 2 \mathrm{H}), 1.89-1.59(\mathrm{~m}, 10 \mathrm{H}), 1.45(\mathrm{~s}, 9 \mathrm{H}), 1.30-$ $1.19(\mathrm{~m}, 2 \mathrm{H}) .{ }^{13} \mathrm{C} \mathrm{NMR}\left(100 \mathrm{MHz}, \mathrm{CDCl}_{3}\right) \delta 170.1,81.7,72.6,65.2,58.5,41.9$, 28.3, 28.0, 26.8, 26.6, 24.8, 23.7, 21.2.IR $\vee 2933,2865,1732,1368,1300$, 1157, 1126, 849, $714 \mathrm{~cm}^{-1}$; HRMS (ESI) m/z: $\left[\mathrm{M}+\mathrm{Na}^{+}\right.$Calcd. for $\mathrm{C}_{17} \mathrm{H}_{32} \mathrm{O}_{5} \mathrm{NaS} 371.1868$; found 371.1855 .

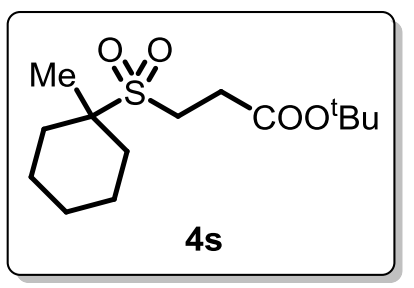

\section{tert-butyl}

propanoate

procedure

1-methylcyclohexane-1-carboxylate $\quad(57.4 \mathrm{mg}, \quad 0.2$ $\mathrm{mmol}$ ), $\mathrm{Na}_{2} \mathrm{~S}_{2} \mathrm{O}_{4}(52.2 \mathrm{mg}, 0.3 \mathrm{mmol})$, tert-Butyl acrylate $(76.9 \mathrm{mg}, 0.6 \mathrm{mmol})$, $\mathrm{Et}_{3} \mathrm{~N} \cdot \mathrm{HCl}(68.8 \mathrm{mg}, 0.5 \mathrm{mmol}), \mathrm{LiOAc}(13.2 \mathrm{mg}, 0.2 \mathrm{mmol})$ and DMF $(2 \mathrm{~mL})$, the reaction was stirred at $100{ }^{\circ} \mathrm{C}$ oil bath for $15 \mathrm{~h}$ giving $4 \mathrm{~s}$ in $70 \%$ yield $(40.4$ $\mathrm{mg})$ as an oil by column chromatography (PE/EA $\left.=5: 1, \mathrm{R}_{\mathrm{f}}=0.3\right) .{ }^{1} \mathrm{H}$ NMR (400 $\left.\mathrm{MHz}, \mathrm{CDCl}_{3}\right) \delta 3.20-3.11(\mathrm{~m}, 2 \mathrm{H}), 2.80-2.76(\mathrm{~m}, 2 \mathrm{H}), 1.91-1.82(\mathrm{~m}, 2 \mathrm{H})$, $1.81-1.67(\mathrm{~m}, 5 \mathrm{H}), 1.44(\mathrm{~s}, 9 \mathrm{H}), 1.43(\mathrm{~s}, 3 \mathrm{H}), 1.27-1.16(\mathrm{~m}, 3 \mathrm{H}) .{ }^{13} \mathrm{C}$ NMR $\left(100 \mathrm{MHz}, \mathrm{CDCl}_{3}\right) \delta 170.0,81.7,62.7,40.8,29.3,28.0,26.6,25.0,21.2$, 17.0.IR $\vee 2979,2866,1730,1368,1305,1154,1138,1094,846,707 \mathrm{~cm}^{-1}$; HRMS (ESI) $\mathrm{m} / \mathrm{z}:[\mathrm{M}+\mathrm{Na}]^{+}$Calcd. for $\mathrm{C}_{14} \mathrm{H}_{26} \mathrm{O}_{4} \mathrm{NaS} 313.1449$; found 313.1436 .

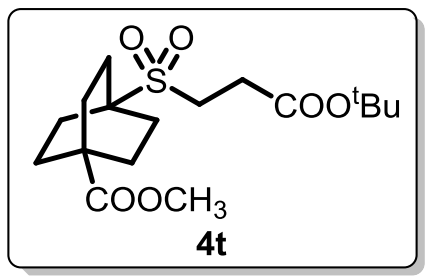

methyl 4-((3-(tert-butoxy)-3-oxopropyl) sulfonyl)bicyclo[2.2.2]octane-1-carboxylate (4t). Prepared following general procedure II using 1-(1,3-dioxoisoindolin-2-yl) 4-methyl bicycle [2.2.2] octane-1,4-dicarboxylate $(71.5 \mathrm{mg}, 0.2 \mathrm{mmol}), \mathrm{Na}_{2} \mathrm{~S}_{2} \mathrm{O}_{4}(52.2 \mathrm{mg}, 0.3 \mathrm{mmol})$, tert-Butyl acrylate $(76.9 \mathrm{mg}, 0.6 \mathrm{mmol}), \mathrm{Et}_{3} \mathrm{~N} \cdot \mathrm{HCl}(68.8 \mathrm{mg}, 0.5 \mathrm{mmol}), \mathrm{LiOAC}$ (13.2 $\mathrm{mg}, 0.2 \mathrm{mmol})$ and DMF $(2 \mathrm{~mL})$, the reaction was stirred at $100{ }^{\circ} \mathrm{C}$ oil bath for $15 \mathrm{~h}$ giving $4 \mathrm{t}$ in $55 \%$ yield $(39.9 \mathrm{mg}$ ) as a white solid by column 
chromatography $\left(\mathrm{PE} / \mathrm{EA}=5: 1, \mathrm{R}_{\mathrm{f}}=0.3\right) .{ }^{1} \mathrm{H}$ NMR $\left(400 \mathrm{MHz}, \mathrm{CDCl}_{3}\right) \delta 3.66$ (s, 3H), $3.14(\mathrm{t}, J=7.8 \mathrm{~Hz}, 2 \mathrm{H}), 2.78(\mathrm{t}, J=7.8 \mathrm{~Hz}, 2 \mathrm{H}), 2.03-1.87(\mathrm{~m}, 12 \mathrm{H}), 1.45$ $(\mathrm{s}, 9 \mathrm{H}) .{ }^{13} \mathrm{C}$ NMR $\left(100 \mathrm{MHz}, \mathrm{CDCl}_{3}\right) \delta 176.6,169.9,81.8,58.9,52.0,41.8,38.3$, 28.0, 27.5, 26.3, 24.1.IR $\vee 2956,2875,1726,1367,1234,1155,1133,1073$, 832, $711 \mathrm{~cm}^{-1}$; HRMS (ESI) m/z: [M + Na] $]^{+}$Calcd. for $\mathrm{C}_{17} \mathrm{H}_{28} \mathrm{O}_{6} \mathrm{NaS} 383.1504$; found 383.1492 .

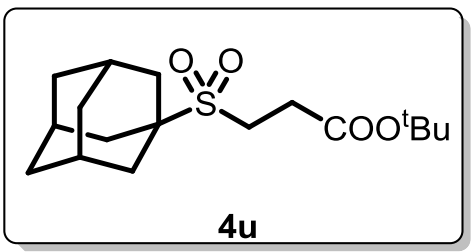

tert-butyl

3-(((3s,5s,7s)-adamantan-1-yl) sulfonyl)propanoate $(\mathbf{4 u})$. Prepared following general procedure II using 1,3-dioxoisoindolin-2-yl adamantane-1-carboxylate $(65.0 \mathrm{mg}, 0.2 \mathrm{mmol})$, $\mathrm{Na}_{2} \mathrm{~S}_{2} \mathrm{O}_{4}(52.2 \mathrm{mg}, 0.3 \mathrm{mmol})$, tert-Butyl acrylate $(76.9 \mathrm{mg}, 0.6 \mathrm{mmol})$, $\mathrm{Et}_{3} \mathrm{~N} \cdot \mathrm{HCl}(68.8 \mathrm{mg}, 0.5 \mathrm{mmol}), \mathrm{LiOAc}(13.2 \mathrm{mg}, 0.2 \mathrm{mmol})$ and DMF $(2 \mathrm{~mL})$, the reaction was stirred at $100{ }^{\circ} \mathrm{C}$ oil bath for $15 \mathrm{~h}$ giving $4 \mathrm{u}$ in $69 \%$ yield $(45.5$ $\mathrm{mg}$ ) as a yellow solid by column chromatography (PE/EA $\left.=5: 1, \mathrm{R}_{\mathrm{f}}=0.3\right) .{ }^{1} \mathbf{H}$ NMR $\left(400 \mathrm{MHz}, \mathrm{CDCl}_{3}\right) \delta 3.17-3.10(\mathrm{~m}, 2 \mathrm{H}), 2.80-2.77(\mathrm{~m}, 2 \mathrm{H}), 2.18(\mathrm{~s}$, 3H), $2.04(\mathrm{~d}, J=2.8 \mathrm{~Hz}, 6 \mathrm{H}), 1.73(\mathrm{q}, J=12.6 \mathrm{~Hz}, 6 \mathrm{H}), 1.45(\mathrm{~s}, 9 \mathrm{H}) .{ }^{13} \mathrm{C}$ NMR $\left(100 \mathrm{MHz}, \mathrm{CDCl}_{3}\right) \delta 170.1,81.7,60.5,40.2,35.8,34.7,28.1,28.0,26.5 . I R \vee$ 2912, 2855, 1732, 1455, 1368, 1302, 1156, 1139, 1048, 837, $714 \mathrm{~cm}^{-1}$; HRMS (ESI) $\mathrm{m} / \mathrm{z}:[\mathrm{M}+\mathrm{Na}]^{+}$Calcd. for $\mathrm{C}_{17} \mathrm{H}_{28} \mathrm{O}_{4} \mathrm{NaS} 351.1606$; found 351.1593 .

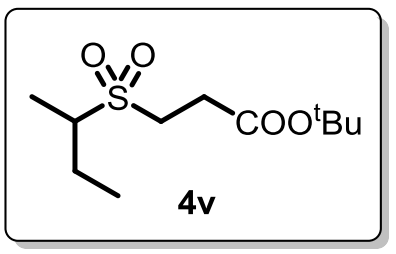

tert-butyl 3-(sec-butylsulfonyl)propanoate (4v). Prepared following general procedure II using 1,3-dioxoisoindolin-2-yl 2-methylbutanoate $(49.5 \mathrm{mg}$, $0.2 \mathrm{mmol}), \mathrm{Na}_{2} \mathrm{~S}_{2} \mathrm{O}_{4}(52.2 \mathrm{mg}, 0.3 \mathrm{mmol})$, tert-Butyl acrylate (76.9 mg, $0.6 \mathrm{mmol}), \mathrm{Et}_{3} \mathrm{~N} \cdot \mathrm{HCl}(68.8 \mathrm{mg}, 0.5 \mathrm{mmol}), \mathrm{LiOAc}(13.2 \mathrm{mg}$, $0.2 \mathrm{mmol}$ ) and DMF (2 mL), the reaction was stirred at $100{ }^{\circ} \mathrm{C}$ oil bath for $15 \mathrm{~h}$ giving $4 \mathbf{v}$ in $51 \%$ yield $(25.6 \mathrm{mg})$ as an oil by column chromatography $(\mathrm{PE} / \mathrm{EA}=$ $\left.5: 1, R_{f}=0.3\right) .{ }^{1} \mathrm{H}$ NMR $\left(400 \mathrm{MHz}, \mathrm{CDCl}_{3}\right) \delta 3.23-3.19(\mathrm{~m}, 2 \mathrm{H}), 2.90-2.82(\mathrm{~m}$, $1 \mathrm{H}), 2.81-2.75(\mathrm{~m}, 2 \mathrm{H}), 1.61-1.53(\mathrm{~m}, 2 \mathrm{H}), 1.46(\mathrm{~s}, 9 \mathrm{H}), 1.40(\mathrm{~d}, J=6.9 \mathrm{~Hz}$, $\mathrm{S} 14$ 
3H), 1.06 (t, $J=7.5 \mathrm{~Hz}, 3 \mathrm{H}) .{ }^{13} \mathrm{C}$ NMR $\left(100 \mathrm{MHz}, \mathrm{CDCl}_{3}\right) \delta 169.8,81.9,59.6$, 44.9, 28.0, 27.3, 22.2, 12.4, 11.2.IR $\vee 2978,2931,1734,1369,1304,1159$, 1122, 846, $722 \mathrm{~cm}^{-1}$; HRMS (ESI) m/z: $[\mathrm{M}+\mathrm{Na}]^{+}$Calcd. for $\mathrm{C}_{11} \mathrm{H}_{22} \mathrm{O}_{4} \mathrm{NaS}$ 273.1136; found 273.1128 .

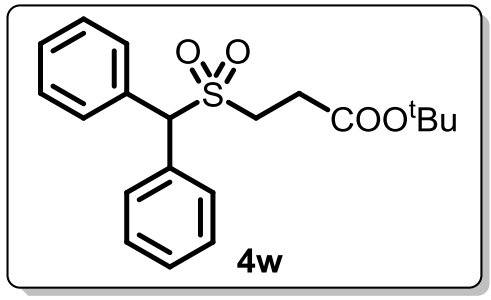

tert-butyl 3-(benzhydrylsulfonyl)propanoate (4w). Prepared following general procedure II using 1,3-dioxoisoindolin-2-yl 2,2-diphenylacetate (71.5 mg, $0.2 \mathrm{mmol}), \mathrm{Na}_{2} \mathrm{~S}_{2} \mathrm{O}_{4}(52.2 \mathrm{mg}, 0.3$ $\mathrm{mmol})$, tert-Butyl acrylate $(76.9 \mathrm{mg}, 0.6 \mathrm{mmol}), \mathrm{Et}_{3} \mathrm{~N} \cdot \mathrm{HCl}(68.8 \mathrm{mg}, 0.5 \mathrm{mmol})$, $\mathrm{LiOAC}(13.2 \mathrm{mg}, 0.2 \mathrm{mmol})$ and DMF $(2 \mathrm{~mL})$, the reaction was stirred at $100^{\circ} \mathrm{C}$ oil bath for $15 \mathrm{~h}$ giving $\mathbf{4 w}$ in $42 \%$ yield $(30.0 \mathrm{mg}$ ) as a white solid by column chromatography $\left(\mathrm{PE} / \mathrm{EA}=5: 1, \mathrm{R}_{\mathrm{f}}=0.3\right) .{ }^{1} \mathrm{H}$ NMR $\left(400 \mathrm{MHz}, \mathrm{CDCl}_{3}\right) \delta 7.66-$ $7.64(\mathrm{~m}, 4 \mathrm{H}), 7.46-7.34(\mathrm{~m}, 6 \mathrm{H}), 5.36(\mathrm{~s}, 1 \mathrm{H}), 3.17-3.10(\mathrm{~m}, 2 \mathrm{H}), 2.75-$ $2.67(\mathrm{~m}, 2 \mathrm{H}), 1.43(\mathrm{~s}, 9 \mathrm{H}) .{ }^{13} \mathrm{C}$ NMR $\left(100 \mathrm{MHz}, \mathrm{CDCl}_{3}\right) \delta 169.5,132.4,129.8$, 129.1, 129.0, 81.9, 73.8, 47.4, 28.0, 27.8.IR $\vee 2982,2928,1730,1496,1453$, 1368, 1316, 1156, 1124, 845, $705 \mathrm{~cm}^{-1}$; HRMS (ESI) m/z: $[\mathrm{M}+\mathrm{Na}]^{+}$Calcd. for $\mathrm{C}_{20} \mathrm{H}_{24} \mathrm{O}_{4} \mathrm{NaS} 383.1293$; found 383.1278 .

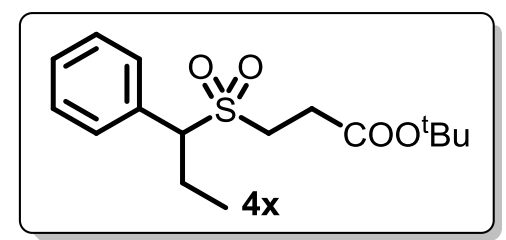

tert-butyl 3-((1-phenylpropyl)sulfonyl) propanoate $(\mathbf{4 x})$. Prepared following general procedure II using 1,3-dioxoisoindolin-2-yl 2-phenylbutanoate $(61.9 \mathrm{mg}, \quad 0.2 \mathrm{mmol})$, $\mathrm{Na}_{2} \mathrm{~S}_{2} \mathrm{O}_{4}(52.2 \mathrm{mg}, 0.3 \mathrm{mmol})$, tert-Butyl acrylate $(76.9 \mathrm{mg}, 0.6 \mathrm{mmol})$, $\mathrm{Et}_{3} \mathrm{~N} \cdot \mathrm{HCl}(68.8 \mathrm{mg}, 0.5 \mathrm{mmol}), \mathrm{LiOAc}(13.2 \mathrm{mg}, 0.2 \mathrm{mmol})$ and DMF $(2 \mathrm{~mL})$, the reaction was stirred at $100{ }^{\circ} \mathrm{C}$ oil bath for $15 \mathrm{~h}$ giving $4 \mathrm{x}$ in $50 \%$ yield $(31.0$ $\mathrm{mg})$ as a yellow solid by column chromatography (PE/EA $\left.=5: 1, \mathrm{R}_{\mathrm{f}}=0.3\right) .{ }^{1} \mathrm{H}$ NMR $\left(400 \mathrm{MHz}, \mathrm{CDCl}_{3}\right) \delta 7.45-7.32(\mathrm{~m}, 5 \mathrm{H}), 3.93(\mathrm{dd}, J=11.3,3.8 \mathrm{~Hz}, 1 \mathrm{H})$, $3.08-2.97(m, 1 H), 2.94-2.86(m, 1 H), 2.70-2.62(m, 1 H), 2.61-2.38(m$, 
2H), $2.15-2.06(\mathrm{~m}, 1 \mathrm{H}), 1.41(\mathrm{~s}, 9 \mathrm{H}), 0.88(\mathrm{t}, J=7.4 \mathrm{~Hz}, 3 \mathrm{H}) .{ }^{13} \mathrm{C}$ NMR $(100$ $\left.\mathrm{MHz}, \mathrm{CDCl}_{3}\right) \delta 169.5,132.4,129.5,129.2,129.1,81.7,70.8,46.1,27.9,27.4$, 20.7, 11.3. IR $\vee 2976,2936,1730,1455,1368,1315,1155,1125,845,702$ $\mathrm{cm}^{-1}$; HRMS (ESI) m/z: $[\mathrm{M}+\mathrm{Na}]^{+}$Calcd. for $\mathrm{C}_{16} \mathrm{H}_{24} \mathrm{O}_{4} \mathrm{NaS}$ 335.1293; found 335.1280 .

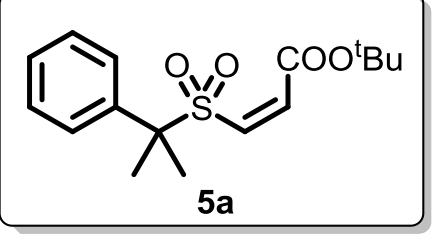

tert-butyl (Z)-3-((2-phenylpropan-2-yl)sulfonyl) acrylate $(\mathbf{5 a})$. Prepared following general procedure III using 1,3-dioxoisoindolin-2-yl 2-methyl-2phenylpropanoate $(61.9 \mathrm{mg}, 0.2 \mathrm{mmol}), \mathrm{Na}_{2} \mathrm{~S}_{2} \mathrm{O}_{4}$ (69.6 mg, $0.4 \mathrm{mmol}$ ), tert-butyl proplolate $(50.5 \mathrm{mg}, 0.4 \mathrm{mmol})$, DIPEA (51.7 mg, $0.4 \mathrm{mmol})$, TBAB (97.6 mg, $0.3 \mathrm{mmol})$, solvent $\left(\mathrm{DMF} / \mathrm{H}_{2} \mathrm{O}=9: 1,2 \mathrm{~mL}\right)$, the reaction was stirred at $40{ }^{\circ} \mathrm{C}$ oil bath for $15 \mathrm{~h}$ giving $5 \mathrm{a}$ in $92 \%$ yield $(57.0$ $\mathrm{mg}, \mathrm{Z} / \mathrm{E}=7: 1)$ as a white solid by column chromatography $\left(\mathrm{PE} / \mathrm{EA}=5: 1, \mathrm{R}_{\mathrm{f}}=\right.$ 0.3). ${ }^{1} \mathrm{H}$ NMR (400 MHz, $\left.\mathrm{CDCl}_{3}\right) \delta 7.63-7.57(\mathrm{~m}, 2 \mathrm{H}), 7.41-7.34(\mathrm{~m}, 3 \mathrm{H})$, $6.45(\mathrm{~d}, J=11.7 \mathrm{~Hz}, 1 \mathrm{H}), 5.82(\mathrm{~d}, J=11.7 \mathrm{~Hz}, 1 \mathrm{H}), 1.85(\mathrm{~s}, 6 \mathrm{H}), 1.54$ (s, 9H). ${ }^{13} \mathrm{C}$ NMR $\left(100 \mathrm{MHz}, \mathrm{CDCl}_{3}\right) \delta 162.9,136.9,135.5,128.6,128.5,128.3$, 126.7, 83.8, 64.9, 28.0, 22.2.IR $\vee 2980,1730,1628,1347,1304,1251,1146$, 1119, 777, $696 \mathrm{~cm}^{-1}$; HRMS (ESI) m/z: $[\mathrm{M}+\mathrm{Na}]^{+}$Calcd. for $\mathrm{C}_{16} \mathrm{H}_{22} \mathrm{O}_{4} \mathrm{NaS}$ 333.1136; found 333.1125 .

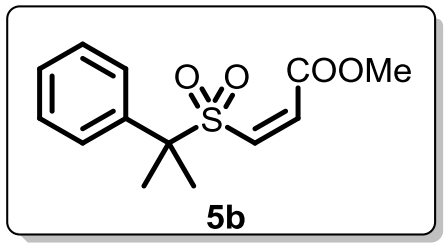

methyl

(Z)-3-((2-phenylpropan-2-yl)sulfonyl) acrylate (5b). Prepared following general procedure III using 1,3-dioxoisoindolin-2-yl 2-methyl-2- phenyl propanoate $(61.9 \mathrm{mg}, 0.2 \mathrm{mmol})$, $\mathrm{Na}_{2} \mathrm{~S}_{2} \mathrm{O}_{4}(69.6 \mathrm{mg}, 0.4 \mathrm{mmol})$, methyl propiolate (33.6 mg, $\left.0.4 \mathrm{mmol}\right)$, DIPEA (51.7 mg, $0.4 \mathrm{mmol})$, TBAB $(97.6 \mathrm{mg}, 0.3 \mathrm{mmol})$, solvent $\left(\mathrm{DMF} / \mathrm{H}_{2} \mathrm{O}=9: 1,2\right.$ $\mathrm{mL}$ ), the reaction was stirred at $40{ }^{\circ} \mathrm{C}$ oil bath for $15 \mathrm{~h}$ giving $5 \mathrm{~b}$ in $50 \%$ yield $(26.1 \mathrm{mg})$ as a white solid by column chromatography (PE/EA $\left.=2: 1, R_{f}=0.2\right)$. ${ }^{1} \mathrm{H}$ NMR $\left(400 \mathrm{MHz}, \mathrm{CDCl}_{3}\right) \delta 7.61-7.58(\mathrm{~m}, 2 \mathrm{H}), 7.49-7.28(\mathrm{~m}, 3 \mathrm{H}), 6.54(\mathrm{~d}$, 
$J=11.7 \mathrm{~Hz}, 1 \mathrm{H}), 5.99(\mathrm{~d}, J=11.7 \mathrm{~Hz}, 1 \mathrm{H}), 3.82(\mathrm{~s}, 3 \mathrm{H}), 1.85(\mathrm{~s}, 6 \mathrm{H}) .{ }^{13} \mathrm{C}$ NMR $\left(100 \mathrm{MHz}, \mathrm{CDCl}_{3}\right) \delta 164.6,136.5,134.4,128.7,128.6,128.5,128.4,65.1$, 52.6, 22.2.IR $\vee 2927,1736,1342,1304,1244,1120,1097,778,700 \mathrm{~cm}^{-1}$; HRMS (ESI) m/z: $[\mathrm{M}+\mathrm{Na}]^{+}$Calcd. for $\mathrm{C}_{13} \mathrm{H}_{16} \mathrm{O}_{4} \mathrm{NaS}$ 291.0667; found 291.0660 .

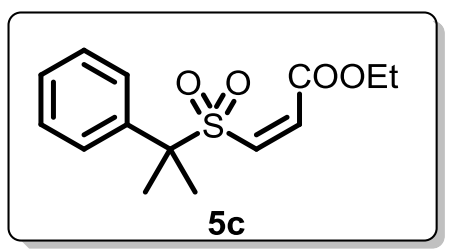

ethyl (Z)-3-((2-phenylpropan-2-yl)sulfonyl) acrylate $(\mathbf{5 c})$. Prepared following general procedure III using 1,3-dioxoisoindolin-2-yl 2-methyl-2- phenyl propanoate $(61.9 \mathrm{mg}, 0.2 \mathrm{mmol}), \mathrm{Na}_{2} \mathrm{~S}_{2} \mathrm{O}_{4}(69.6 \mathrm{mg}$, $0.4 \mathrm{mmol}$ ), ethyl propiolate (39.2 $\mathrm{mg}, 0.4 \mathrm{mmol})$, DIPEA (51.7 $\mathrm{mg}, 0.4 \mathrm{mmol})$, TBAB (97.6 $\mathrm{mg}, 0.3 \mathrm{mmol})$, solvent $\left(\mathrm{DMF} / \mathrm{H}_{2} \mathrm{O}=9: 1,2 \mathrm{~mL}\right)$, the reaction was stirred at $40{ }^{\circ} \mathrm{C}$ oil bath for $15 \mathrm{~h}$ giving $5 \mathrm{c}$ in $82 \%$ yield $(46.3 \mathrm{mg}, \mathrm{Z} / \mathrm{E}=7: 1)$ as an oil by column chromatography $\left(P E / E A=2: 1, R_{f}=0.4\right) .{ }^{1} H$ NMR $(400 \mathrm{MHz}$, $\left.\mathrm{CDCl}_{3}\right) \delta 7.61-7.59(\mathrm{~m}, 2 \mathrm{H}), 7.42-7.35(\mathrm{~m}, 3 \mathrm{H}), 6.54(\mathrm{~d}, \mathrm{~J}=11.7 \mathrm{~Hz}, 1 \mathrm{H})$, $5.95(\mathrm{~d}, J=11.7 \mathrm{~Hz}, 1 \mathrm{H}), 4.29(\mathrm{q}, J=7.1 \mathrm{~Hz}, 2 \mathrm{H}), 1.84(\mathrm{~s}, 6 \mathrm{H}), 1.33$ (t, $J=7.2$ $\mathrm{Hz}, 3 \mathrm{H}) .{ }^{13} \mathrm{C}$ NMR $\left(100 \mathrm{MHz}, \mathrm{CDCl}_{3}\right) \delta 164.1,136.6,134.7,128.7,128.5,128.3$, 128.2, 65.0, 61.9, 22.2, 13.9.IR $\vee 2988,1735,1369,1303,1236,1156,1120$, 1097, 1022, 777, $697 \mathrm{~cm}^{-1}$; HRMS (ESI) m/z: $\left[\mathrm{M}+\mathrm{Na}^{+}\right.$Calcd. for $\mathrm{C}_{14} \mathrm{H}_{18} \mathrm{O}_{4} \mathrm{NaS}$ 305.0823; found 305.0814 .

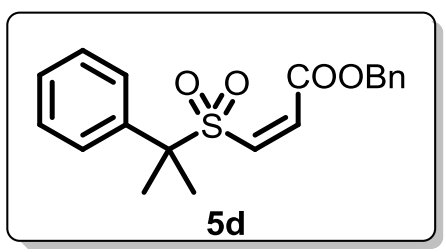

benzyl (Z)-3-((2-phenylpropan-2-yl)sulfonyl) acrylate (5d). Prepared following general procedure III using 1,3-dioxoisoindolin-2-yl 2-methyl-2- phenyl propanoate $(61.9 \mathrm{mg}, 0.2 \mathrm{mmol})$, $\mathrm{Na}_{2} \mathrm{~S}_{2} \mathrm{O}_{4}(69.6 \mathrm{mg}, 0.4 \mathrm{mmol})$, benzyl propiolate $(64.1 \mathrm{mg}, 0.4 \mathrm{mmol})$, DIPEA (51.7 mg, $0.4 \mathrm{mmol})$, TBAB (97.6 mg, $0.3 \mathrm{mmol})$, solvent $\left(\mathrm{DMF} / \mathrm{H}_{2} \mathrm{O}=9: 1,2\right.$ $\mathrm{mL}$ ), the reaction was stirred at $40{ }^{\circ} \mathrm{C}$ oil bath for $15 \mathrm{~h}$ giving $5 \mathrm{~d}$ in $68 \%$ yield (46.8 $\mathrm{mg}, \mathrm{Z} / \mathrm{E}=6: 1)$ as an oil by column chromatography $\left(\mathrm{PE} / \mathrm{EA}=2: 1, \mathrm{R}_{\mathrm{f}}=\right.$ 0.4). ${ }^{1} \mathrm{H}$ NMR $\left(400 \mathrm{MHz}, \mathrm{CDCl}_{3}\right) \delta 7.60-7.57(\mathrm{~m}, 2 \mathrm{H}), 7.46-7.31(\mathrm{~m}, 8 \mathrm{H})$, 
$6.56(\mathrm{~d}, J=11.7 \mathrm{~Hz}, 1 \mathrm{H}), 5.99(\mathrm{~d}, J=11.7 \mathrm{~Hz}, 1 \mathrm{H}), 5.26(\mathrm{~s}, 2 \mathrm{H}), 1.83$ (s, 6H). ${ }^{13} \mathrm{C}$ NMR $\left(100 \mathrm{MHz}, \mathrm{CDCl}_{3}\right) \delta 164.0,136.5,134.8,134.4,134.3,128.8$, 128.7, 128.6, 128.5(3), 128.5(1), 128.4, 67.8, 65.0, 22.2.IR 2367, 1735, 1499, 1341, 1304, 1226, 1156, 1120, 777, $697 \mathrm{~cm}^{-1}$; HRMS (ESI) m/z: $[\mathrm{M}+\mathrm{Na}]^{+}$ Calcd. for $\mathrm{C}_{19} \mathrm{H}_{20} \mathrm{O}_{4} \mathrm{NaS} 367.0980$; found 367.0971 .

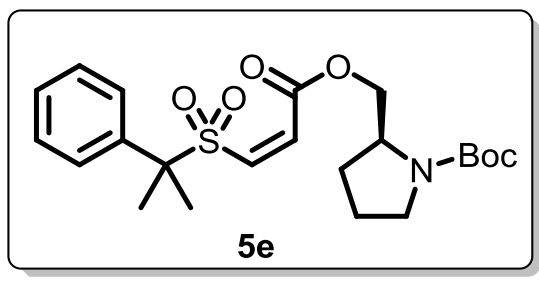

tert-butyl (S,Z)-2-(((3-)(2-phenylpropan-2yl)sulfonyl)acryloyl)oxy)methyl)pyrrolidine1-carboxylate (5e). Prepared following general procedure III using 1,3-dioxoisoindolin-2-yl 2-methyl-2- phenyl propanoate $(61.9 \mathrm{mg}, 0.2 \mathrm{mmol}), \mathrm{Na}_{2} \mathrm{~S}_{2} \mathrm{O}_{4}(69.6 \mathrm{mg}, 0.4 \mathrm{mmol})$, tert-butyl (S)-2-((propioloyloxy)methyl) pyrrolidine-1-carboxylate $(101.3 \mathrm{mg}, 0.4 \mathrm{mmol})$, DIPEA (51.7 mg, $0.4 \mathrm{mmol})$, TBAB $(97.6 \mathrm{mg}, 0.3 \mathrm{mmol})$, solvent $\left(\mathrm{DMF} / \mathrm{H}_{2} \mathrm{O}=\right.$ $9: 1,2 \mathrm{~mL}$ ), the reaction was stirred at $40{ }^{\circ} \mathrm{C}$ oil bath for $15 \mathrm{~h}$ giving $5 \mathrm{e}$ in $72 \%$ yield $(62.9 \mathrm{mg}, \mathrm{Z} / \mathrm{E}=8: 1)$ as an oil by column chromatography $(\mathrm{PE} / \mathrm{EA}=2: 1$, $\left.\mathrm{R}_{\mathrm{f}}=0.4\right) .{ }^{1} \mathrm{H}$ NMR $\left(400 \mathrm{MHz}, \mathrm{CDCl}_{3}\right) \delta 7.59-7.57(\mathrm{~m}, 2 \mathrm{H}), 7.41-7.35(\mathrm{~m}, 3 \mathrm{H})$, $6.54(\mathrm{~d}, J=11.7 \mathrm{~Hz}, 1 \mathrm{H}), 5.95(\mathrm{~d}, J=11.7 \mathrm{~Hz}, 1 \mathrm{H}), 4.36-3.99(\mathrm{~m}, 3 \mathrm{H}), 3.39-$ $3.28(\mathrm{~m}, 2 \mathrm{H}), 2.06-1.83(\mathrm{~m}, 4 \mathrm{H}), 1.82(\mathrm{~s}, 6 \mathrm{H}), 1.46(\mathrm{~s}, 9 \mathrm{H}) .{ }^{13} \mathrm{C}$ NMR $(100$ $\left.\mathrm{MHz}, \mathrm{CDCl}_{3}\right) \delta 164.0,154.3,136.5,134.7,134.2,128.7,128.5,128.3,79.7$, 79.3, 65.9, 64.9, 55.2, 46.7, 46.4, 28.8, 28.4, 27.8, 23.7, 22.9, 22.2, 22.1.IR v 2974, 1740, 1690, 1393, 1305, 1120, 776, $697 \mathrm{~cm}^{-1}$; HRMS (ESI) m/z: [M + $\mathrm{Na}]^{+}$Calcd. for $\mathrm{C}_{22} \mathrm{H}_{31} \mathrm{NO}_{6} \mathrm{NaS} 460.1770$; found 460.1761 .

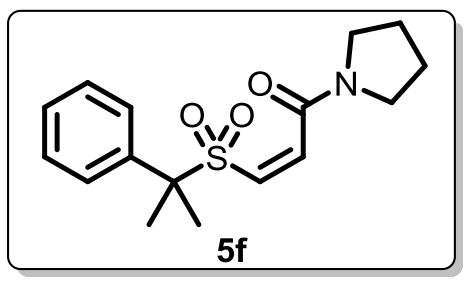

(Z)-3-((2-phenylpropan-2-yl)sulfonyl) $-1-$ (pyrrolidin-1-yl)prop-2-en-1-one (5f). Prepared following general procedure III using 1,3-dioxoisoindolin-2-yl 2-methyl-2- phenyl propanoate $(61.9 \mathrm{mg}, 0.2 \mathrm{mmol}), \mathrm{Na}_{2} \mathrm{~S}_{2} \mathrm{O}_{4}(69.6 \mathrm{mg}, 0.4 \mathrm{mmol})$, 1-(pyrrolidin-1-yl)prop-2-yn-1-one (49.3 mg, $0.4 \mathrm{mmol}$ ), DIPEA (51.7 mg, 0.4 mmol), TBAB (97.6 mg, $0.3 \mathrm{mmol})$, solvent $\left(\mathrm{DMF} / \mathrm{H}_{2} \mathrm{O}=9: 1,2 \mathrm{~mL}\right)$, the 
reaction was stirred at $40{ }^{\circ} \mathrm{C}$ oil bath for $15 \mathrm{~h}$ giving $5 \mathrm{f}$ in $50 \%$ yield $(30.0 \mathrm{mg})$ as a white solid by column chromatography $\left(\mathrm{DCM} / \mathrm{MeOH}=20: 1, \mathrm{R}_{\mathrm{f}}=0.4\right) .{ }^{1} \mathrm{H}$ NMR $\left(400 \mathrm{MHz}, \mathrm{CDCl}_{3}\right) \delta 7.61-7.58(\mathrm{~m}, 2 \mathrm{H}), 7.40-7.33(\mathrm{~m}, 3 \mathrm{H}), 6.65$ (d, $J=$ $11.8 \mathrm{~Hz}, 1 \mathrm{H}), 5.81(\mathrm{~d}, J=11.8 \mathrm{~Hz}, 1 \mathrm{H}), 3.52-3.48(\mathrm{~m}, 2 \mathrm{H}), 3.36-3.30(\mathrm{~m}$, $2 \mathrm{H}), 1.91-1.86(\mathrm{~m}, 4 \mathrm{H}), 1.84(\mathrm{~s}, 6 \mathrm{H}) \cdot{ }^{13} \mathrm{C}$ NMR $\left(100 \mathrm{MHz}, \mathrm{CDCl}_{3}\right) \delta 162.8$, 138.6, 136.6, 128.6, 128.5, 128.4, 125.5, 64.9, 47.4, 45.2, 25.5, 24.1, 22.2.IR v 2926, 1636, 1441, 1300, 1120, 1097, 779, $699 \mathrm{~cm}^{-1}$; HRMS (ESI) m/z: [M + H] $]^{+}$ Calcd. for $\mathrm{C}_{16} \mathrm{H}_{22} \mathrm{NO}_{3} \mathrm{~S} 308.1320$; found 308.1313 .

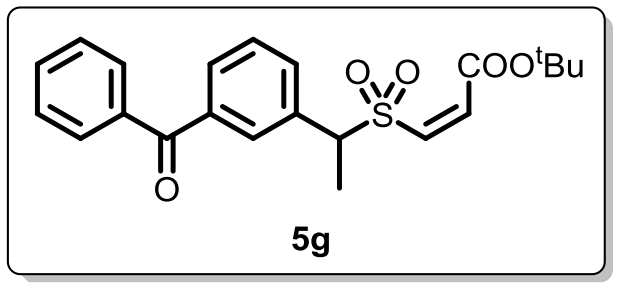

\section{tert-butyl (Z)-3-((1-(3-benzoylphenyl)} ethyl)sulfonyl)acrylate $(\mathbf{5 g})$. Prepared following general procedure III using 1,3-dioxoisoindolin-2-yl 2-(3-benzoylphenyl) propanoate $(79.9 \mathrm{mg}, 0.2 \mathrm{mmol}), \mathrm{Na}_{2} \mathrm{~S}_{2} \mathrm{O}_{4}$ (69.6 mg, $0.4 \mathrm{mmol})$, tert-butyl proplolate $(50.5 \mathrm{mg}, 0.4 \mathrm{mmol})$, DIPEA (51.7 $\mathrm{mg}, 0.4 \mathrm{mmol})$, TBAB (97.6 mg, $0.3 \mathrm{mmol})$, solvent $\left(\mathrm{DMF} / \mathrm{H}_{2} \mathrm{O}=9: 1,2 \mathrm{~mL}\right)$, the reaction was stirred at $40{ }^{\circ} \mathrm{C}$ oil bath for $15 \mathrm{~h}$ giving $5 \mathrm{~g}$ in $61 \%$ yield $(49.0$ $\mathrm{mg}$ ) as an oil by column chromatography (PE/EA = 2:1, $\left.\mathrm{R}_{\mathrm{f}}=0.3\right) .{ }^{1} \mathrm{H}$ NMR (400 $\left.\mathrm{MHz}, \mathrm{CDCl}_{3}\right) \delta 7.85-7.84(\mathrm{~m}, 1 \mathrm{H}), 7.81-7.78(\mathrm{~m}, 3 \mathrm{H}), 7.69(\mathrm{~d}, J=7.9 \mathrm{~Hz}$, $1 \mathrm{H}), 7.61-7.57(\mathrm{~m}, 1 \mathrm{H}), 7.52-7.46(\mathrm{~m}, 3 \mathrm{H}), 6.51(\mathrm{~d}, J=11.6 \mathrm{~Hz}, 1 \mathrm{H}), 6.21(\mathrm{~d}$ $J=11.6 \mathrm{~Hz}, 1 \mathrm{H}), 4.71(\mathrm{q}, J=7.2 \mathrm{~Hz}, 1 \mathrm{H}), 1.80(\mathrm{~d}, J=7.2 \mathrm{~Hz}, 3 \mathrm{H}), 1.53(\mathrm{~s}$, 9H). ${ }^{13} \mathrm{C}$ NMR $\left(100 \mathrm{MHz}, \mathrm{CDCl}_{3}\right) \delta 195.9,162.6,138.0,137.1,136.2,133.7$, $133.3,133.1,132.7,131.2,130.6,130.1,128.8,128.3,84.1,64.8,27.9$, 13.6.IR $\vee 2982,1728,1660,1349,1317,1152,1127,750,702 \mathrm{~cm}^{-1}$; HRMS (ESI) $\mathrm{m} / \mathrm{z}:[\mathrm{M}+\mathrm{Na}]^{+}$Calcd. for $\mathrm{C}_{22} \mathrm{H}_{24} \mathrm{O}_{5} \mathrm{NaS} 423.1242$; found 423.1234 .

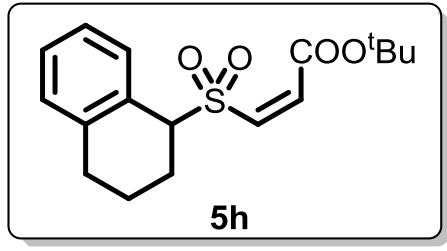

(64.3mg, $0.2 \mathrm{mmol}), \mathrm{Na}_{2} \mathrm{~S}_{2} \mathrm{O}_{4}(69.6 \mathrm{mg}, 0.4 \mathrm{mmol})$, tert-butyl proplolate $(50.5$ tert-butyl (Z)-3-((1,2,3,4-tetrahydronaphthalen -1-yl)sulfonyl)acrylate (5h). Prepared following general procedure III using 1,3-dioxoisoindolin-2-yl 1,2,3,4-tetrahydronaphthalene -1-carboxylate 
$\mathrm{mg}, 0.4 \mathrm{mmol})$, DIPEA (51.7 mg, $0.4 \mathrm{mmol}), \operatorname{TBAB}(97.6 \mathrm{mg}, 0.3 \mathrm{mmol})$, solvent $\left(\mathrm{DMF} / \mathrm{H}_{2} \mathrm{O}=9: 1,2 \mathrm{~mL}\right)$, the reaction was stirred at $40^{\circ} \mathrm{C}$ oil bath for 15 $\mathrm{h}$ giving $5 \mathrm{~h}$ in $71 \%$ yield $(45.7 \mathrm{mg}, \mathrm{Z} / \mathrm{E}=5: 1)$ as a white solid by column chromatography (PE/EA = 5:1, $\left.\mathrm{R}_{\mathrm{f}}=0.3\right) .{ }^{1} \mathrm{H}$ NMR $\left(400 \mathrm{MHz}, \mathrm{CDCl}_{3}\right) \delta 7.53$ (d, $J=7.6 \mathrm{~Hz}, 1 \mathrm{H}), 7.28-7.24(\mathrm{~m}, 1 \mathrm{H}), 7.20-7.15(\mathrm{~m}, 2 \mathrm{H}), 6.53(\mathrm{~d}, J=11.6 \mathrm{~Hz}$, $1 \mathrm{H}), 6.32(\mathrm{~d}, J=11.6 \mathrm{~Hz}, 1 \mathrm{H}), 4.62(\mathrm{dd}, J=6.8,3.7 \mathrm{~Hz}, 1 \mathrm{H}), 2.93-2.85(\mathrm{~m}$, $1 \mathrm{H}), 2.80-2.71(\mathrm{~m}, 1 \mathrm{H}), 2.61-2.53(\mathrm{~m}, 1 \mathrm{H}), 2.31-2.21(\mathrm{~m}, 1 \mathrm{H}), 2.18-2.09$ $(\mathrm{m}, 1 \mathrm{H}), 1.76-1.69(\mathrm{~m}, 1 \mathrm{H}), 1.56(\mathrm{~s}, 9 \mathrm{H}) .{ }^{13} \mathrm{C}$ NMR $\left(100 \mathrm{MHz}, \mathrm{CDCl}_{3}\right) \delta 162.9$, 139.8, 135.2, 132.9, 131.4, 129.6, 128.7, 126.2, 125.9, 84.0, 63.9, 28.7, 27.9, 23.3, 19.1.IR $\vee 2979,1729,1349,1312,1253,1152,1125,779,749 \mathrm{~cm}^{-1}$; HRMS (ESI) m/z: $[\mathrm{M}+\mathrm{Na}]^{+}$Calcd. for $\mathrm{C}_{17} \mathrm{H}_{22} \mathrm{O}_{4} \mathrm{NaS}$ 345.1136; found 345.1128.

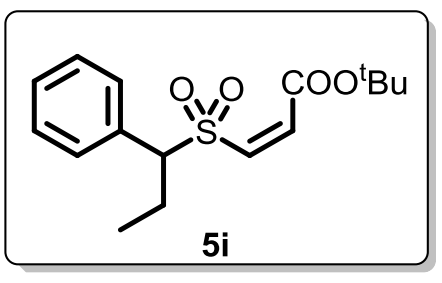

tert-butyl

(Z)-3-((1-phenylpropyl)sulfonyl) acrylate $(5 i)$. Prepared following general procedure III using 1,3-dioxoisoindolin-2-yl 2-phenylbutanoate (61.9mg, $0.2 \mathrm{mmol}$ ), $\mathrm{Na}_{2} \mathrm{~S}_{2} \mathrm{O}_{4}$ (69.6 mg, $0.4 \mathrm{mmol}$ ), tert-butyl proplolate $(50.5 \mathrm{mg}, 0.4 \mathrm{mmol})$, DIPEA ( $51.7 \mathrm{mg}, 0.4 \mathrm{mmol}$ ), TBAB (97.6 $\mathrm{mg}, 0.3 \mathrm{mmol})$, solvent $\left(\mathrm{DMF} / \mathrm{H}_{2} \mathrm{O}=9: 1,2 \mathrm{~mL}\right)$, the reaction was stirred at $40{ }^{\circ} \mathrm{C}$ oil bath for $15 \mathrm{~h}$ giving $5 \mathrm{i}$ in $79 \%$ yield $(49.0 \mathrm{mg}, \mathrm{Z} / \mathrm{E}=6: 1)$ as an oil by column chromatography $\left(\mathrm{PE} / \mathrm{EA}=5: 1, \mathrm{R}_{\mathrm{f}}=0.3\right) .{ }^{1} \mathrm{H} \mathbf{N M R}\left(400 \mathrm{MHz}, \mathrm{CDCl}_{3}\right) \delta$ $7.43-7.34(\mathrm{~m}, 5 \mathrm{H}), 6.38(\mathrm{~d}, J=11.7 \mathrm{~Hz}, 1 \mathrm{H}), 6.04(\mathrm{~d}, J=11.7 \mathrm{~Hz}, 1 \mathrm{H}), 4.34$ (dd, $J=11.2,4.0 \mathrm{~Hz}, 1 \mathrm{H}), 2.48-2.39(\mathrm{~m}, 1 \mathrm{H}), 2.19-2.08(\mathrm{~m}, 1 \mathrm{H}), 1.56(\mathrm{~s}$, $9 \mathrm{H}), 0.90$ (t, $J=7.4 \mathrm{~Hz}, 3 \mathrm{H}) .{ }^{13} \mathrm{C}$ NMR $\left(100 \mathrm{MHz}, \mathrm{CDCl}_{3}\right) \delta$ 162.8, 135.1, 133.8, $131.9,130.0,129.0,128.8,83.9,72.1,28.0,20.6,11.2 . I R \vee 2975,2936,1728$, 1455, 1315, 1251, 1152, 1127, $704 \mathrm{~cm}^{-1}$; HRMS (ESI) m/z: $[\mathrm{M}+\mathrm{Na}]^{+}$Calcd. for $\mathrm{C}_{16} \mathrm{H}_{22} \mathrm{O}_{4} \mathrm{NaS} 333.1136$; found 333.1128. 


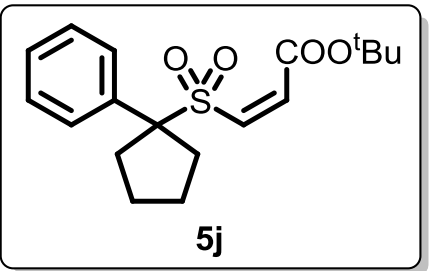

tert-butyl (Z)-3-((1-phenylcyclopentyl)sulfonyl) acrylate (5j). Prepared following general procedure III using 1,3-dioxoisoindolin-2-yl 1-phenylcyclopentane-1-carboxylate $\quad(67.1 \mathrm{mg}, \quad 0.2$ mmol), $\mathrm{Na}_{2} \mathrm{~S}_{2} \mathrm{O}_{4}$ (69.6 mg, $0.4 \mathrm{mmol}$ ), tert-butyl proplolate $(50.5 \mathrm{mg}, 0.4 \mathrm{mmol})$, DIPEA $(51.7 \mathrm{mg}, 0.4 \mathrm{mmol})$, TBAB $(97.6 \mathrm{mg}$, $0.3 \mathrm{mmol})$, solvent $\left(\mathrm{DMF} / \mathrm{H}_{2} \mathrm{O}=9: 1,2 \mathrm{~mL}\right)$, the reaction was stirred at $40^{\circ} \mathrm{C}$ oil bath for $15 \mathrm{~h}$ giving $5 \mathbf{j}$ in $88 \%$ yield $(59.2 \mathrm{mg}$ ) as a white solid by column chromatography (PE/EA $\left.=5: 1, \mathrm{R}_{\mathrm{f}}=0.3\right) .{ }^{1} \mathrm{H}$ NMR $\left(400 \mathrm{MHz}, \mathrm{CDCl}_{3}\right) \delta 7.57-$ $7.46(\mathrm{~m}, 2 \mathrm{H}), 7.41-7.30(\mathrm{~m}, 3 \mathrm{H}), 6.41(\mathrm{~d}, J=11.7 \mathrm{~Hz}, 1 \mathrm{H}), 5.75(\mathrm{~d}, J=11.7$ $\mathrm{Hz}, 1 \mathrm{H}), 2.97-2.83(\mathrm{~m}, 2 \mathrm{H}), 2.37-2.29(\mathrm{~m}, 2 \mathrm{H}), 2.08-1.93(\mathrm{~m}, 2 \mathrm{H}), 1.77-$ $1.66(\mathrm{~m}, 2 \mathrm{H}), 1.53(\mathrm{~s}, 9 \mathrm{H}) .{ }^{13} \mathrm{C}$ NMR $\left(100 \mathrm{MHz}, \mathrm{CDCl}_{3}\right) \delta 163.2,137.6,134.8$, 129.6, 128.5, 128.3, 127.5, 83.7, 76.8, 33.8, 27.9, 24.9.IR $\vee 2921,1724,1630$, 1450, 1347, 1305, 1155, 1120, 850, $786 \mathrm{~cm}^{-1}$; HRMS (ESI) m/z: $[\mathrm{M}+\mathrm{Na}]^{+}$ Calcd. for $\mathrm{C}_{18} \mathrm{H}_{24} \mathrm{O}_{4} \mathrm{NaS} 359.1293$; found 359.1283 .

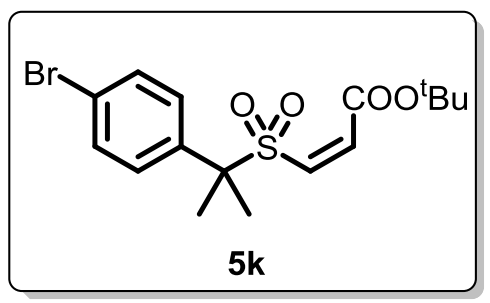

tert-butyl (Z)-3-((2-(4-bromophenyl)propan -2-yl)sulfonyl)acrylate (5k). Prepared following general procedure III using 1,3-dioxoisoindolin-2-yl 2-(4-bromophenyl)-2methylpropanoate $(77.6 \mathrm{mg}, 0.2 \mathrm{mmol}), \mathrm{Na}_{2} \mathrm{~S}_{2} \mathrm{O}_{4}$ (69.6 mg, $0.4 \mathrm{mmol})$, tert-butyl proplolate $(50.5 \mathrm{mg}, 0.4 \mathrm{mmol})$, DIPEA (51.7 $\mathrm{mg}, 0.4 \mathrm{mmol})$, TBAB (97.6 mg, $0.3 \mathrm{mmol})$, solvent $\left(\mathrm{DMF} / \mathrm{H}_{2} \mathrm{O}=9: 1,2 \mathrm{~mL}\right)$, the reaction was stirred at $40{ }^{\circ} \mathrm{C}$ oil bath for $15 \mathrm{~h}$ giving $5 \mathbf{k}$ in $80 \%$ yield $(62.3$ $\mathrm{mg}, \mathrm{Z} / \mathrm{E}=8: 1)$ as a white solid by column chromatography $\left(\mathrm{PE} / \mathrm{EA}=5: 1, \mathrm{R}_{\mathrm{f}}=\right.$ 0.3). ${ }^{1} \mathrm{H}$ NMR $\left(400 \mathrm{MHz}, \mathrm{CDCl}_{3}\right) \delta 7.53-7.49(\mathrm{~m}, 2 \mathrm{H}), 7.49-7.43(\mathrm{~m}, 2 \mathrm{H})$, $6.50(\mathrm{~d}, J=11.7 \mathrm{~Hz}, 1 \mathrm{H}), 5.86(\mathrm{~d}, J=11.7 \mathrm{~Hz}, 1 \mathrm{H}), 1.81(\mathrm{~s}, 6 \mathrm{H}), 1.53(\mathrm{~s}$, 9H).$^{13} \mathrm{C}$ NMR $\left(100 \mathrm{MHz}, \mathrm{CDCl}_{3}\right) \delta 162.8,136.1,135.9,131.6,130.1,126.3$, 123.2, 83.9, 64.56, 27.9, 22.3.IR $\vee 2981,1731,1348,1304,1254,1150,1120$, 1096, 1009, $740 \mathrm{~cm}^{-1}$; HRMS (ESI) m/z: $[\mathrm{M}+\mathrm{Na}]^{+}$Calcd. for $\mathrm{C}_{16} \mathrm{H}_{21} \mathrm{BrO}_{4} \mathrm{NaS}$ 411.0242, 413.0221; found 411.0231, 413.0208. 


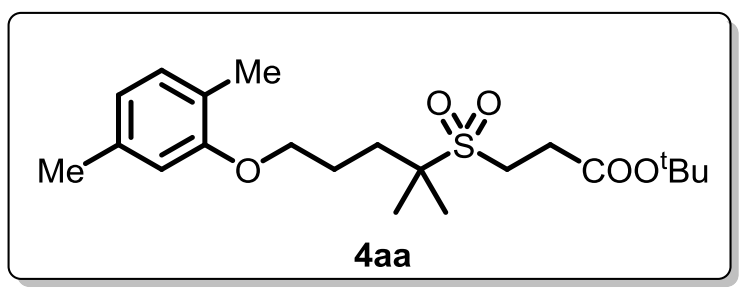

tert-butyl

3-((5-(2,5-dimethyl

phenoxy)-2-methylpentan-2-yl)

sulfonyl)propanoate

(4aa).

Prepared following general

procedure II using redox-active ester derived from Gemfibrozil $(71.5 \mathrm{mg}, 0.2$ mmol), $\mathrm{Na}_{2} \mathrm{~S}_{2} \mathrm{O}_{4}(52.2 \mathrm{mg}, 0.3 \mathrm{mmol})$, tert-Butyl acrylate $(76.9 \mathrm{mg}, 0.6 \mathrm{mmol})$, $\mathrm{Et}_{3} \mathrm{~N} \cdot \mathrm{HCl}(68.8 \mathrm{mg}, 0.5 \mathrm{mmol}), \mathrm{LiOAC}(13.2 \mathrm{mg}, 0.2 \mathrm{mmol})$ and DMF $(2 \mathrm{~mL})$, the reaction was stirred at $100{ }^{\circ} \mathrm{C}$ oil bath for $15 \mathrm{~h}$ giving 4 aa in $65 \%$ yield $(51.5$ $\mathrm{mg}$ ) as a white solid by column chromatography (PE/EA $\left.=5: 1, \mathrm{R}_{\mathrm{f}}=0.3\right) .{ }^{1} \mathbf{H}$ NMR $\left(400 \mathrm{MHz}, \mathrm{CDCl}_{3}\right) \delta 7.01(\mathrm{~d}, J=7.5 \mathrm{~Hz}, 1 \mathrm{H}), 6.67(\mathrm{~d}, J=7.5 \mathrm{~Hz}, 1 \mathrm{H})$, $6.61(\mathrm{~s}, 1 \mathrm{H}), 3.97(\mathrm{t}, J=5.7 \mathrm{~Hz}, 2 \mathrm{H}), 3.27-3.19(\mathrm{~m}, 2 \mathrm{H}), 2.85-2.80(\mathrm{~m}, 2 \mathrm{H})$, $2.31(\mathrm{~s}, 3 \mathrm{H}), 2.18(\mathrm{~s}, 3 \mathrm{H}), 2.04-1.88(\mathrm{~m}, 4 \mathrm{H}), 1.47(\mathrm{~s}, 9 \mathrm{H}), 1.44(\mathrm{~s}, 6 \mathrm{H}) .{ }^{13} \mathrm{C}$ NMR $\left(100 \mathrm{MHz}, \mathrm{CDCl}_{3}\right) \delta 169.9,156.6,136.5,130.4,123.5,120.9,112.0,81.8$, 67.4, 62.0, 41.6, 32.0, 28.0, 26.6, 24.1, 21.3, 20.6, 15.8.IR $\vee 2977,1730,1616$, 1586, 1509, 1368, 1265, 1155, 1111, 805, $719 \mathrm{~cm}^{-1}$; HRMS (ESI) m/z: [M + $\mathrm{Na}]^{+}$Calcd. for $\mathrm{C}_{21} \mathrm{H}_{34} \mathrm{O}_{5} \mathrm{NaS}$ 421.2025; found 421.2012.

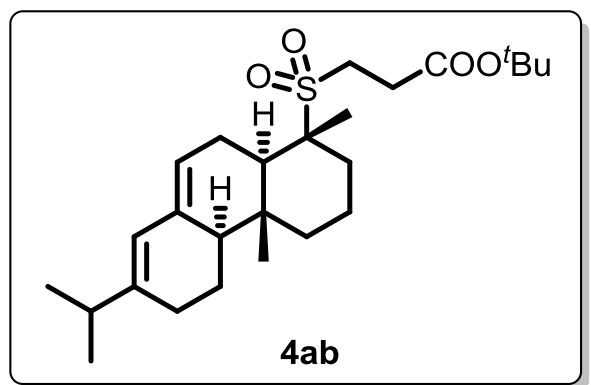

tert-butyl

3-(((1R,4aR,4bR,10aR)-7isopropyl-1,4a-dimethyl-1,2,3,4,4a,4b,5,6 ,10,10a-decahydrophenanthren-1-yl)sulf onyl)propanoate (4ab). Prepared following general procedure II using redox-active ester derived from Abietic acid (89.5 mg, $0.2 \mathrm{mmol}), \mathrm{Na}_{2} \mathrm{~S}_{2} \mathrm{O}_{4}(52.2 \mathrm{mg}, 0.3 \mathrm{mmol})$, tert-Butyl acrylate (76.9 $\mathrm{mg}, 0.6 \mathrm{mmol}), \mathrm{Et}_{3} \mathrm{~N} \cdot \mathrm{HCl}(68.8 \mathrm{mg}, 0.5 \mathrm{mmol}), \mathrm{LiOAC}(13.2 \mathrm{mg}, 0.2 \mathrm{mmol})$ and DMF (2 mL), the reaction was stirred at $100{ }^{\circ} \mathrm{C}$ oil bath for $15 \mathrm{~h}$ giving $4 \mathrm{ab}$ in $44 \%$ yield $(39.4 \mathrm{mg})$ as an oil by column chromatography $\left(\mathrm{PE} / \mathrm{EA}=10: 1, \mathrm{R}_{\mathrm{f}}=\right.$ 0.3). ${ }^{1} \mathrm{H}$ NMR $\left(400 \mathrm{MHz}, \mathrm{CDCl}_{3}\right) \delta 5.77(\mathrm{~s}, 1 \mathrm{H}), 5.39-5.32(\mathrm{~m}, 1 \mathrm{H}), 3.25-$ $3.19(\mathrm{~m}, 1 \mathrm{H}), 3.15-3.07(\mathrm{~m}, 1 \mathrm{H}), 2.80-2.75(\mathrm{~m}, 2 \mathrm{H}), 2.60(\mathrm{~d}, J=17.6 \mathrm{~Hz}$, 
1H), $2.28-2.15(\mathrm{~m}, 3 \mathrm{H}), 2.07(\mathrm{~d}, J=6.4 \mathrm{~Hz}, 2 \mathrm{H}), 1.92-1.76(\mathrm{~m}, 5 \mathrm{H}), 1.52(\mathrm{~s}$, $3 \mathrm{H}), 1.45(\mathrm{~s}, 11 \mathrm{H}), 1.26-1.22(\mathrm{~m}, 2 \mathrm{H}), 1.00(\mathrm{dd}, J=6.8,3.6 \mathrm{~Hz}, 6 \mathrm{H}), 0.86$ (s, $3 \mathrm{H}) .{ }^{13} \mathrm{C}$ NMR $\left(100 \mathrm{MHz}, \mathrm{CDCl}_{3}\right) \delta 170.2,145.3,134.7,122.2,120.1,81.7$, $67.4,50.5,41.8,41.7,37.6,35.9,34.8,34.3,28.0,27.3,26.7,25.3,22.6,21.4$, 20.8, 18.2, 15.3, 14.2.IR v 2959, 2938, 1730, 1458, 1368, 1155, 1122, 845, $701 \mathrm{~cm}^{-1} ;$ HRMS (ESI) m/z: [M + Na] $]^{+}$Calcd. for $\mathrm{C}_{26} \mathrm{H}_{42} \mathrm{O}_{4} \mathrm{NaS} 473.2702$; found 473.2700.

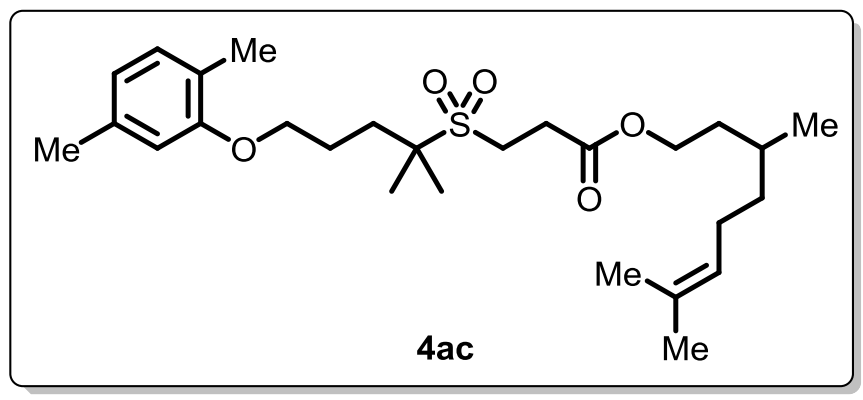

3,7-dimethyloct-6-en-1-yl 3-((5-(2,5-dimethylphenoxy )-2-methylpentan-2-yl)sulfo nyl)propanoate (4ac). Prepared following general procedure II using redox-active ester derived from Gemfibrozil (71.5 mg, $0.2 \mathrm{mmol}$ ), $\mathrm{Na}_{2} \mathrm{~S}_{2} \mathrm{O}_{4}$ (52.2 mg, $0.3 \mathrm{mmol}$ ), 3,7-dimethyloct-6-en-1-yl acrylate (126.2 mg, $0.6 \mathrm{mmol}$ ), $\mathrm{Et}_{3} \mathrm{~N} \cdot \mathrm{HCl}(68.8 \mathrm{mg}, 0.5 \mathrm{mmol}), \mathrm{LiOAC}(13.2 \mathrm{mg}, 0.2 \mathrm{mmol})$ and DMF $(2 \mathrm{~mL})$, the reaction was stirred at $100{ }^{\circ} \mathrm{C}$ oil bath for $15 \mathrm{~h}$ giving $4 \mathrm{ac}$ in $45 \%$ yield $(43.6$ $\mathrm{mg})$ as an oil by column chromatography (PE/EA $\left.=5: 1, \mathrm{R}_{\mathrm{f}}=0.4\right) .{ }^{1} \mathrm{H}$ NMR (400 $\left.\mathrm{MHz}, \mathrm{CDCl}_{3}\right) \delta 7.00(\mathrm{~d}, J=7.5 \mathrm{~Hz}, 1 \mathrm{H}), 6.67(\mathrm{~d}, J=7.5 \mathrm{~Hz}, 1 \mathrm{H}), 6.61(\mathrm{~s}, 1 \mathrm{H})$, $5.11-5.06(\mathrm{~m}, 1 \mathrm{H}), 4.19-4.13(\mathrm{~m}, 2 \mathrm{H}), 3.97(\mathrm{t}, J=5.7 \mathrm{~Hz}, 2 \mathrm{H}), 3.31-3.23$ $(\mathrm{m}, 2 \mathrm{H}), 2.95-2.85(\mathrm{~m}, 2 \mathrm{H}), 2.31(\mathrm{~s}, 3 \mathrm{H}), 2.17(\mathrm{~s}, 3 \mathrm{H}), 2.06-1.86(\mathrm{~m}, 6 \mathrm{H})$, $1.68(\mathrm{~s}, 3 \mathrm{H}), 1.60(\mathrm{~s}, 3 \mathrm{H}), 1.57-1.52(\mathrm{~m}, 1 \mathrm{H}), 1.51-1.46(\mathrm{~m}, 1 \mathrm{H}), 1.44(\mathrm{~s}$, $6 \mathrm{H}), 1.37-1.14(\mathrm{~m}, 3 \mathrm{H}), 0.91(\mathrm{~d}, \mathrm{~J}=6.5 \mathrm{~Hz}, 3 \mathrm{H}) .{ }^{13} \mathbf{C ~ N M R}\left(100 \mathrm{MHz}, \mathrm{CDCl}_{3}\right)$ ठ 170.9, 156.7, 136.6, 131.4, 130.4, 124.5, 123.5, 121.0, 112.0, 67.4, 64.0, 62.1, 41.6, 37.0, 35.3, 32.1, 29.5, 25.7, 25.6, 25.4, 24.2, 21.4, 20.7, 19.4, 17.7, 15.8.IR v 2873, 1736, 1509, 1460, 1266, 1130, 1044, 804, $722 \mathrm{~cm}^{-1} ;$ HRMS (ESI) m/z: $[\mathrm{M}+\mathrm{H}]^{+}$Calcd. for $\mathrm{C}_{27} \mathrm{H}_{45} \mathrm{O}_{5} \mathrm{~S}$ 481.2988; found 481.2978. 


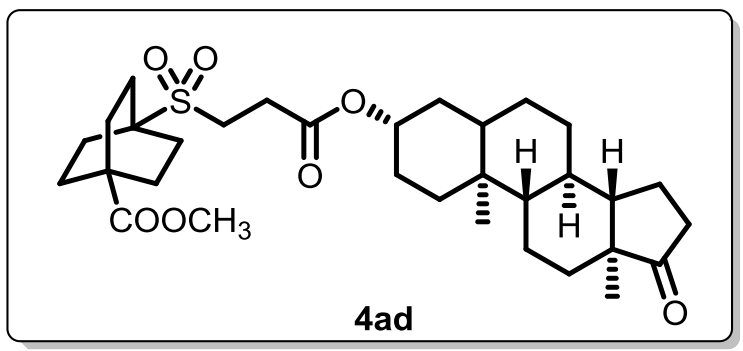

methyl 4-((3-(((3S,8R,9S,10S,13S, 14S)-10,13-dimethyl-17-oxohexad ecahydro-1H-cyclopenta[a]phena nthren-3-yl)oxy)-3-oxopropyl)sulf onyl)bicyclo[2.2.2]octane-1-carb

oxylate (4ad). Prepared following general procedure II using 1-(1,3-dioxoisoindolin-2-yl) 4-methyl bicycle [2.2.2] octane-1,4-dicarboxylate (71.5 mg, $0.2 \mathrm{mmol}), \mathrm{Na}_{2} \mathrm{~S}_{2} \mathrm{O}_{4}(52.2 \mathrm{mg}, 0.3 \mathrm{mmol})$, olefin michael acceptor derived from Epiandrosterone (206.7 mg, $0.6 \mathrm{mmol}$ ), $\mathrm{Et}_{3} \mathrm{~N} \cdot \mathrm{HCl}(68.8 \mathrm{mg}, 0.5$ $\mathrm{mmol})$, LiOAc (13.2 $\mathrm{mg}, 0.2 \mathrm{mmol})$ and DMF $(2 \mathrm{~mL})$, the reaction was stirred at $100{ }^{\circ} \mathrm{C}$ oil bath for $15 \mathrm{~h}$ giving $4 \mathrm{ad}$ in $55 \%$ yield $(63.1 \mathrm{mg})$ as a yellow solid by column chromatography $\left(\mathrm{PE} / \mathrm{EA}=2: 1, \mathrm{R}_{\mathrm{f}}=0.4\right) .{ }^{1} \mathrm{H} \mathbf{N M R}\left(400 \mathrm{MHz}, \mathrm{CDCl}_{3}\right) \delta$ $4.74-4.65(\mathrm{~m}, 1 \mathrm{H}), 3.63(\mathrm{~s}, 3 \mathrm{H}), 3.16(\mathrm{t}, J=7.7 \mathrm{~Hz}, 2 \mathrm{H}), 2.80(\mathrm{t}, J=7.7 \mathrm{~Hz}$, 2H), $2.40(\mathrm{dd}, J=19.2,8.7 \mathrm{~Hz}, 1 \mathrm{H}), 1.99-1.87(\mathrm{~m}, 12 \mathrm{H}), 1.82-1.69(\mathrm{~m}, 6 \mathrm{H})$, $1.63-1.44(\mathrm{~m}, 6 \mathrm{H}), 1.30-1.20(\mathrm{~m}, 6 \mathrm{H}), 1.03-0.93(\mathrm{~m}, 2 \mathrm{H}), 0.83(\mathrm{~s}, 6 \mathrm{H})$, $0.71-0.64(\mathrm{~m}, 1 \mathrm{H}) .{ }^{13} \mathrm{C}$ NMR $\left(100 \mathrm{MHz}, \mathrm{CDCl}_{3}\right) \delta 221.1,176.5,170.2,74.7$, 58.9, 54.2, 51.9, 51.3, 47.7, 44.6, 41.8, 38.3, 36.6, 35.7, 35.5, 34.9, 33.7, 31.4, $30.7,28.2,27.4,27.2,25.6,24.1,21.7,20.4,13.7,12.1 . I R \vee 2949,1734,1716$, 1361, 1220, 1134, 1014, 828, $616 \mathrm{~cm}^{-1}$; HRMS (ESI) m/z: $[\mathrm{M}+\mathrm{H}]^{+}$Calcd. for $\mathrm{C}_{32} \mathrm{H}_{49} \mathrm{O}_{7} \mathrm{~S}$ 577.3199; found 577.3192.

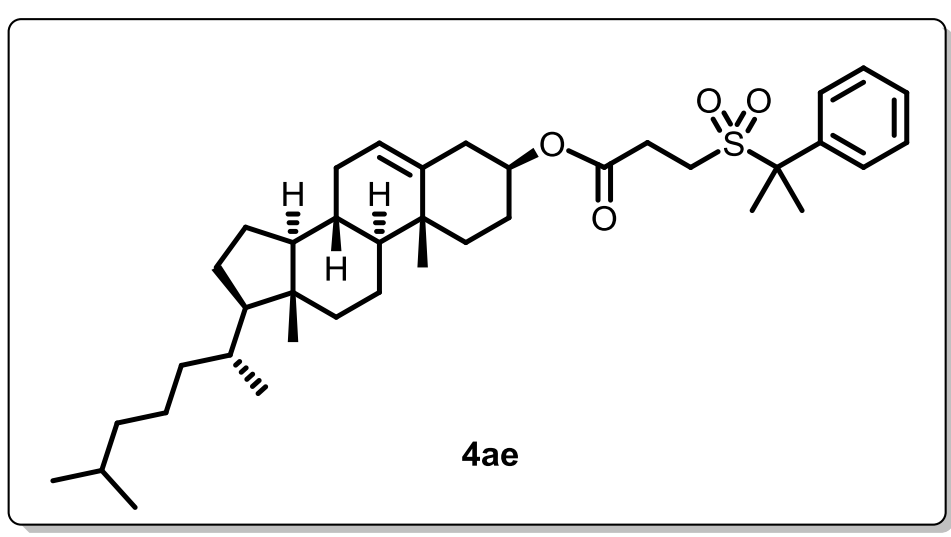

3-yl 3-((2-phenylpropan-2-yl)sulfonyl)propanoate

$(3 S, 8 S, 9 S, 10 R, 13 R, 14$ S,17R)-10,13-dimethyl17-((R)-6-methylhepta n-2-yl)-2,3,4,7,8,9,10,1 $1,12,13,14,15,16,17$-tet radecahydro-1 $\mathrm{H}$-cyclo penta[a]phenanthren(4ae). Prepared following general procedure II using 1,3-dioxoisoindolin- 2-yl 2- methyl-2- 
phenyl propanoate $(61.9 \mathrm{mg}, 0.2 \mathrm{mmol}), \mathrm{Na}_{2} \mathrm{~S}_{2} \mathrm{O}_{4}(52.2 \mathrm{mg}, 0.3 \mathrm{mmol})$, olefin michael acceptor derived from Cholesterol (264.4 mg, $0.6 \mathrm{mmol}$ ), $\mathrm{Et}_{3} \mathrm{~N} \cdot \mathrm{HCl}$ (68.8 $\mathrm{mg}, 0.5 \mathrm{mmol})$, LiOAC (13.2 $\mathrm{mg}, 0.2 \mathrm{mmol})$ and DMF (2 mL), the reaction was stirred at $100{ }^{\circ} \mathrm{C}$ oil bath for $15 \mathrm{~h}$ giving $4 \mathrm{ae}$ in $60 \%$ yield $(74.8 \mathrm{mg})$ as a white solid by column chromatography (PE/EA $\left.=5: 1, R_{f}=0.2\right) .{ }^{1} \mathbf{H}$ NMR (400 $\left.\mathrm{MHz}, \mathrm{CDCl}_{3}\right) \delta 7.65-7.63(\mathrm{~m}, 2 \mathrm{H}), 7.43-7.36(\mathrm{~m}, 3 \mathrm{H}), 5.35$ (d, J=4.4 Hz, $1 \mathrm{H}), 4.60-4.52(\mathrm{~m}, 1 \mathrm{H}), 2.98-2.89(\mathrm{~m}, 2 \mathrm{H}), 2.62-2.53(\mathrm{~m}, 2 \mathrm{H}), 2.26(\mathrm{~d}, J=$ $7.7 \mathrm{~Hz}, 2 \mathrm{H}), 2.04-1.93(\mathrm{~m}, 2 \mathrm{H}), 1.86(\mathrm{~s}, 6 \mathrm{H}), 1.84-1.77(\mathrm{~m}, 2 \mathrm{H}), 1.61-1.40$ $(\mathrm{m}, 8 \mathrm{H}), 1.39-1.21(\mathrm{~m}, 6 \mathrm{H}), 1.19-1.04(\mathrm{~m}, 8 \mathrm{H}), 1.00(\mathrm{~s}, 3 \mathrm{H}), 0.91(\mathrm{~d}, J=6.5$ $\mathrm{Hz}, 3 \mathrm{H}), 0.87-0.85(\mathrm{~m}, 6 \mathrm{H}), 0.67(\mathrm{~s}, 3 \mathrm{H}) .{ }^{13} \mathrm{C} \mathrm{NMR}\left(100 \mathrm{MHz}, \mathrm{CDCl}_{3}\right) \delta 170.0$, $139.4,137.1,128.7(3), 128.6(6), 128.0,122.8,75.0,65.0,56.7,56.1,50.0$, 42.3, 42.0, 39.7, 39.5, 37.9, 36.9, 36.5, 36.2, 35.8, 31.9, 31.8, 28.2, 28.0, 27.6, 26.2, 24.2, 23.8, 22.8, 22.5, 22.4, 21.0, 19.3, 18.7, 11.8.IR v 2956, 2856, 1738, 1469, 1379, 1303, 1191, 1118, 778, $698 \mathrm{~cm}^{-1}$; HRMS (ESI) m/z: $[\mathrm{M}+\mathrm{Na}]^{+}$ Calcd. for $\mathrm{C}_{39} \mathrm{H}_{60} \mathrm{O}_{4} \mathrm{NaS}$ 647.4110; found 647.4097.

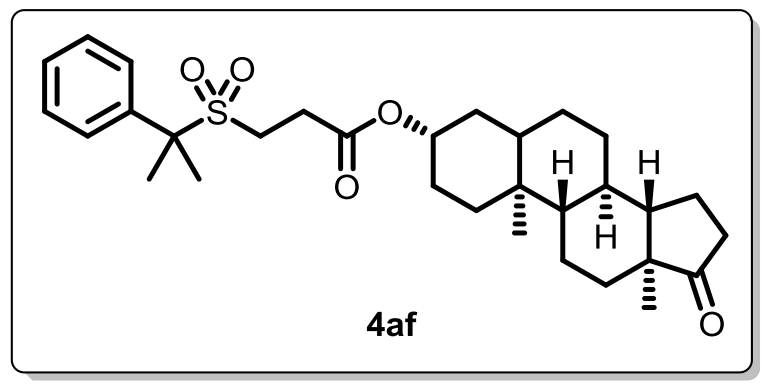

(3S,8R,9S,10S,13S,14S)-10,13-di methyl-17-oxohexadecahydro-1H -cyclopenta[a]phenanthren-3-yl 3-((2-phenylpropan-2-yl) sulfonyl) propanoate (4af). Prepared

following general procedure II using 1,3-dioxoisoindolin- 2-yl 2- methyl-2phenyl propanoate $(61.9 \mathrm{mg}, 0.2 \mathrm{mmol}), \mathrm{Na}_{2} \mathrm{~S}_{2} \mathrm{O}_{4}(52.2 \mathrm{mg}, 0.3 \mathrm{mmol})$, olefin michael acceptor derived from Epiandrosterone (206.7 mg, $0.6 \mathrm{mmol})$, $\mathrm{Et}_{3} \mathrm{~N} \cdot \mathrm{HCl}(68.8 \mathrm{mg}, 0.5 \mathrm{mmol})$, LiOAc (13.2 mg, $\left.0.2 \mathrm{mmol}\right)$ and DMF (2 mL), the reaction was stirred at $100{ }^{\circ} \mathrm{C}$ oil bath for $15 \mathrm{~h}$ giving 4 af in $60 \%$ yield $(63.5$ $\mathrm{mg}$ ) as a white solid by column chromatography (PE/EA = 2:1, $\left.\mathrm{R}_{\mathrm{f}}=0.2\right) .{ }^{1} \mathrm{H}$ NMR $\left(400 \mathrm{MHz}, \mathrm{CDCl}_{3}\right) \delta 7.63-7.61(\mathrm{~m}, 2 \mathrm{H}), 7.45-7.30(\mathrm{~m}, 3 \mathrm{H}), 4.71-4.54$ (m, 1H), 2.91 (t, $J=7.7 \mathrm{~Hz}, 2 \mathrm{H}$ ), $2.54(\mathrm{t}, J=7.7 \mathrm{~Hz}, 2 \mathrm{H}$ ), 2.41 (dd, $J=19.2,8.8$ 
$\mathrm{Hz}, 1 \mathrm{H}), 2.09-2.00(\mathrm{~m}, 1 \mathrm{H}), 1.94-1.88(\mathrm{~m}, 1 \mathrm{H}), 1.85(\mathrm{~s}, 6 \mathrm{H}), 1.81-1.71(\mathrm{~m}$, $4 \mathrm{H}), 1.58-1.43(\mathrm{~m}, 4 \mathrm{H}), 1.36-1.14(\mathrm{~m}, 8 \mathrm{H}), 1.03-0.91(\mathrm{~m}, 2 \mathrm{H}), 0.82(\mathrm{~d}, J=$ $7.9 \mathrm{~Hz}, 6 \mathrm{H}), 0.71-0.64(\mathrm{~m}, 1 \mathrm{H}) .{ }^{13} \mathrm{C}$ NMR $\left(100 \mathrm{MHz}, \mathrm{CDCl}_{3}\right) \delta$ 221.0, 170.1, 137.0, 128.7, 128.6, 128.0, 74.5, 64.9, 54.2, 51.3, 47.7, 44.5, 42.0, 36.5, 35.7, 35.5, 34.9, 33.7, 31.4, 30.7, 28.2, 27.2, 26.1, 22.4, 21.7, 20.4, 13.7, 12.1. IR v 2943, 2855, 1735, 1364, 1302, 1120, 1098, 777, $699 \mathrm{~cm}^{-1}$; HRMS (ESI) m/z: $[\mathrm{M}+\mathrm{Na}]^{+}$Calcd. for $\mathrm{C}_{31} \mathrm{H}_{44} \mathrm{O}_{5} \mathrm{NaS} 551.2807$; found 551.2796.

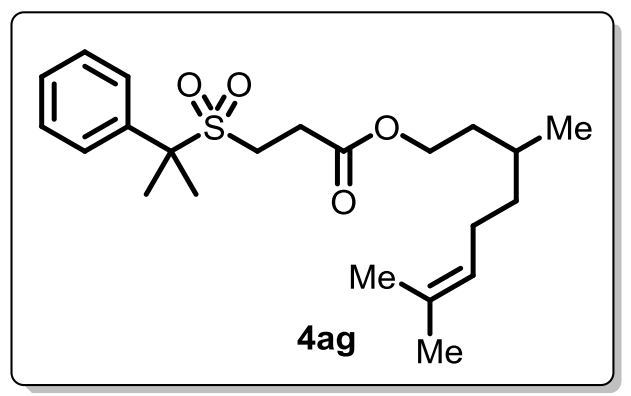

3,7-dimethyloct-6-en-1-yl 3-((2-phenyl propan-2-yl)sulfonyl)propanoate (4ag). Prepared following general procedure II using 1,3-dioxoisoindolin- 2-yl 2- methyl-2phenyl propanoate $(61.9 \mathrm{mg}, 0.2 \mathrm{mmol})$, $\mathrm{Na}_{2} \mathrm{~S}_{2} \mathrm{O}_{4}(52.2 \mathrm{mg}, 0.3 \mathrm{mmol})$, olefin michael acceptor derived from Citronellol (126.2 mg, $0.6 \mathrm{mmol}), \mathrm{Et}_{3} \mathrm{~N} \cdot \mathrm{HCl}$ (68.8 $\mathrm{mg}, 0.5 \mathrm{mmol})$, LiOAC (13.2 $\mathrm{mg}, 0.2 \mathrm{mmol})$ and DMF $(2 \mathrm{~mL})$, the reaction was stirred at $100{ }^{\circ} \mathrm{C}$ oil bath for $15 \mathrm{~h}$ giving $4 \mathrm{ag}$ in $62 \%$ yield $(49.1 \mathrm{mg})$ as an oil by column chromatography (PE/EA = 10:1, $\left.\mathrm{R}_{\mathrm{f}}=0.2\right) .{ }^{1} \mathbf{H} \mathbf{N M R}(400 \mathrm{MHz}$, $\left.\mathrm{CDCl}_{3}\right) \delta 7.68-7.58(\mathrm{~m}, 2 \mathrm{H}), 7.44-7.33(\mathrm{~m}, 3 \mathrm{H}), 5.06(\mathrm{t}, J=7.0 \mathrm{~Hz}, 1 \mathrm{H}), 4.10$ - $4.01(\mathrm{~m}, 2 \mathrm{H}), 2.98-2.87(\mathrm{~m}, 2 \mathrm{H}), 2.63-2.53(\mathrm{~m}, 2 \mathrm{H}), 1.99-1.92(\mathrm{~m}, 2 \mathrm{H})$, $1.86(\mathrm{~s}, 6 \mathrm{H}), 1.67(\mathrm{~s}, 3 \mathrm{H}), 1.59(\mathrm{~s}, 3 \mathrm{H}), 1.52-1.26(\mathrm{~m}, 4 \mathrm{H}), 1.20-1.12(\mathrm{~m}$, $1 \mathrm{H}), 0.87(\mathrm{~d}, J=6.5 \mathrm{~Hz}, 3 \mathrm{H}) .{ }^{13} \mathrm{C}$ NMR $\left(100 \mathrm{MHz}, \mathrm{CDCl}_{3}\right) \delta 170.7,137.1,131.4$, 128.8, 128.7, 128.1, 124.5, 65.1, 63.9, 42.0, 36.9, 35.3, 29.4, 25.9, 25.7, 25.4, 22.4, 19.4, 17.7.IR $\vee 2964,2915,1736,1450,1304,1120,1098,777,699 \mathrm{~cm}^{-1}$; HRMS (ESI) m/z: $[\mathrm{M}+\mathrm{Na}]^{+}$Calcd. for $\mathrm{C}_{22} \mathrm{H}_{34} \mathrm{O}_{4} \mathrm{NaS}$ 417.2075; found 417.2064. 


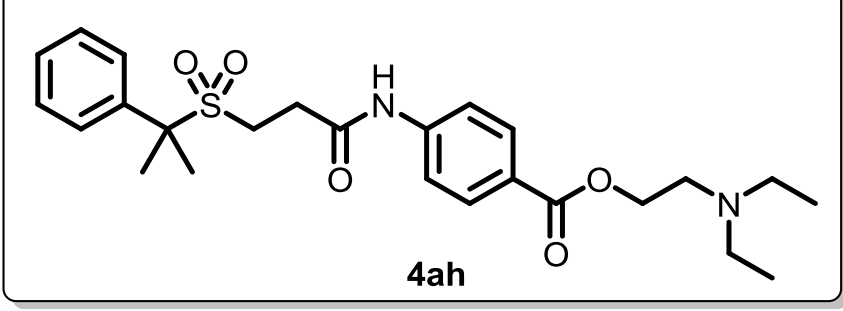

2-(diethylamino)ethyl 4-(3((2-phenylpropan-2-yl)sulfo nyl)propanamido)benzoate (4ah). Prepared following general procedure II using 1,3-dioxoisoindolin- 2-yl 2- methyl-2- phenyl propanoate $(61.9 \mathrm{mg}, 0.2 \mathrm{mmol}$ ), $\mathrm{Na}_{2} \mathrm{~S}_{2} \mathrm{O}_{4}(52.2 \mathrm{mg}, 0.3 \mathrm{mmol})$, olefin michael acceptor derived from Procaine (174.2 mg, $0.6 \mathrm{mmol}), \mathrm{Et}_{3} \mathrm{~N} \cdot \mathrm{HCl}(68.8 \mathrm{mg}, 0.5 \mathrm{mmol})$, LiOAc (13.2 mg, 0.2 $\mathrm{mmol})$ and DMF $(2 \mathrm{~mL})$, the reaction was stirred at $100{ }^{\circ} \mathrm{C}$ oil bath for $15 \mathrm{~h}$ giving 4 ah in $54 \%$ yield $(50.9 \mathrm{mg})$ as a yellow solid by column chromatography $\left(\mathrm{DCM} / \mathrm{MeOH}=20: 1, \mathrm{R}_{\mathrm{f}}=0.2\right) .{ }^{1} \mathrm{H}$ NMR $\left(400 \mathrm{MHz}, \mathrm{CDCl}_{3}\right) \delta 8.78(\mathrm{~s}, 1 \mathrm{H}), 7.91$ $(\mathrm{d}, J=8.7 \mathrm{~Hz}, 2 \mathrm{H}), 7.63-7.60(\mathrm{~m}, 2 \mathrm{H}), 7.53(\mathrm{~d}, J=8.7 \mathrm{~Hz}, 2 \mathrm{H}), 7.40-7.33$ (m, 3H), $4.44(\mathrm{t}, J=6.0 \mathrm{~Hz}, 2 \mathrm{H}$ ), $3.09(\mathrm{t}, J=8.0 \mathrm{~Hz}, 2 \mathrm{H}), 2.97$ (t, $J=6.0 \mathrm{~Hz}$, $2 \mathrm{H}), 2.82-2.69(\mathrm{~m}, 6 \mathrm{H}), 1.87(\mathrm{~s}, 6 \mathrm{H}), 1.12(\mathrm{t}, J=7.2 \mathrm{~Hz}, 6 \mathrm{H}) .{ }^{13} \mathrm{C}$ NMR $(100$ $\left.\mathrm{MHz}, \mathrm{CDCl}_{3}\right) \delta 168.0,165.9,142.3,136.5,130.7,128.9,128.7,128.0,125.2$, 118.8, 65.2, 62.0, 50.5, 47.4, 41.9, 28.0, 22.4, 11.0. IR $\vee 3336,2969,1709$, 1600, 1535, 1409, 1273, 1117, 771, $697 \mathrm{~cm}^{-1}$; HRMS (ESI) m/z: [M + H] Calcd. for $\mathrm{C}_{25} \mathrm{H}_{35} \mathrm{~N}_{2} \mathrm{O}_{5} \mathrm{~S} 475.2267$; found 475.2255 .

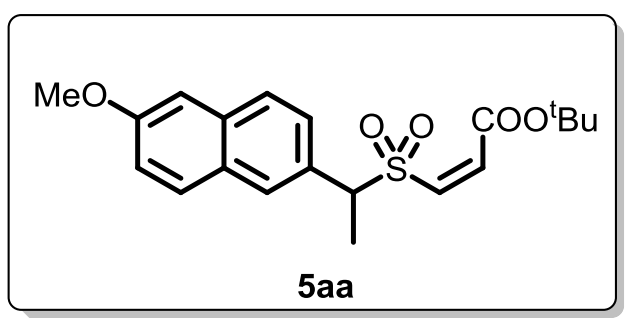

tert-butyl (Z)-3-((1-(6-methoxynaphth alen-2-yl)ethyl)sulfonyl)acrylate (5aa). Prepared following general procedure III using redox-active ester derived from Naproxen $(75.1 \mathrm{mg}, 0.2 \mathrm{mmol}), \mathrm{Na}_{2} \mathrm{~S}_{2} \mathrm{O}_{4}$ (69.6 mg, $0.4 \mathrm{mmol}$ ), tert-butyl proplolate $(50.5 \mathrm{mg}, 0.4 \mathrm{mmol})$, DIPEA (51.7 mg, $0.4 \mathrm{mmol})$, TBAB (97.6 mg, $0.3 \mathrm{mmol})$, solvent $\left(\mathrm{DMF} / \mathrm{H}_{2} \mathrm{O}=9: 1,2 \mathrm{~mL}\right)$, the reaction was stirred at $40^{\circ} \mathrm{C}$ oil bath for $15 \mathrm{~h}$ giving $5 \mathrm{aa}$ in $77 \%$ yield $(58.0$ $\mathrm{mg}, \mathrm{Z} / \mathrm{E}=6: 1)$ as an oil by column chromatography $\left(\mathrm{PE} / \mathrm{EA}=5: 1, \mathrm{R}_{\mathrm{f}}=0.3\right) .{ }^{1} \mathrm{H}$ NMR $\left(400 \mathrm{MHz}, \mathrm{CDCl}_{3}\right) \delta 7.81(\mathrm{~s}, 1 \mathrm{H}), 7.73(\mathrm{~d}, J=8.6 \mathrm{~Hz}, 2 \mathrm{H}), 7.53-7.50(\mathrm{~m}$, $1 \mathrm{H}), 7.19-7.09(\mathrm{~m}, 2 \mathrm{H}), 6.41(\mathrm{~d}, J=11.7 \mathrm{~Hz}, 1 \mathrm{H}), 6.09(\mathrm{~d}, J=11.7 \mathrm{~Hz}, 1 \mathrm{H})$, 4.74 (q, $J=7.2 \mathrm{~Hz}, 1 \mathrm{H}$ ), $3.92(\mathrm{~s}, 3 \mathrm{H}), 1.86(\mathrm{~d}, J=7.2 \mathrm{~Hz}, 3 \mathrm{H}), 1.58(\mathrm{~s}, 9 \mathrm{H}) \cdot{ }^{13} \mathrm{C}$ 
NMR $\left(100 \mathrm{MHz}, \mathrm{CDCl}_{3}\right) \delta 162.9,158.3,135.4,134.7,133.5,129.6,128.9$, 128.6, 128.5, 127.3, 127.0, 119.4, 105.5, 83.9, 65.3, 55.3, 28.0, 13.5.IR $\vee 2981$, 2937, 1729, 1607, 1632, 1349, 1313, 1152, 1126, 854, $742 \mathrm{~cm}^{-1}$; HRMS (ESI) $\mathrm{m} / \mathrm{z}:[\mathrm{M}+\mathrm{Na}]^{+}$Calcd. for $\mathrm{C}_{20} \mathrm{H}_{24} \mathrm{O}_{5} \mathrm{NaS} 399.1242$; found 399.1230 .

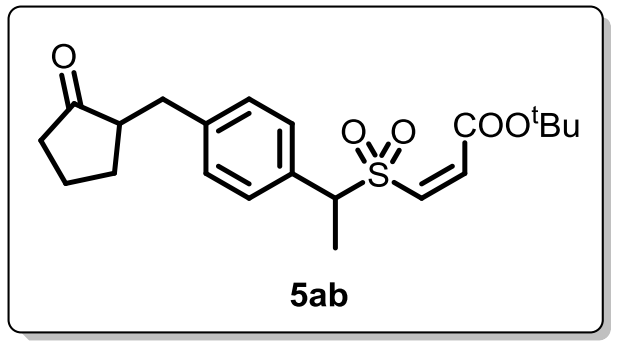

tert-butyl(Z)-3-((1-(4-((2-oxocyclopentyl) methyl)phenyl)ethyl)sulfonyl) acrylate (5ab). Prepared following general procedure III using redox-active ester derived from Loxoprofen $(78.3 \mathrm{mg}, \quad 0.2$ $\mathrm{mmol}), \mathrm{Na}_{2} \mathrm{~S}_{2} \mathrm{O}_{4}(69.6 \mathrm{mg}, 0.4 \mathrm{mmol})$, tert-butyl proplolate $(50.5 \mathrm{mg}, 0.4 \mathrm{mmol})$, DIPEA (51.7 mg, $0.4 \mathrm{mmol})$, TBAB (97.6 $\mathrm{mg}, 0.3 \mathrm{mmol})$, solvent $\left(\mathrm{DMF} / \mathrm{H}_{2} \mathrm{O}=9: 1,2 \mathrm{~mL}\right)$, the reaction was stirred at $40{ }^{\circ} \mathrm{C}$ oil bath for $15 \mathrm{~h}$ giving $5 \mathrm{ab}$ in $57 \%$ yield $(44.7 \mathrm{mg}, \mathrm{Z} / \mathrm{E}=7: 1)$ as an oil by column chromatography $\left(\mathrm{PE} / \mathrm{EA}=2: 1, \mathrm{R}_{\mathrm{f}}=0.2\right) .{ }^{1} \mathrm{H} \mathbf{N M R}\left(400 \mathrm{MHz}, \mathrm{CDCl}_{3}\right)$ $\delta 7.33(\mathrm{~d}, J=8.1 \mathrm{~Hz}, 2 \mathrm{H}), 7.15(\mathrm{~d}, J=8.1 \mathrm{~Hz}, 2 \mathrm{H}), 6.45(\mathrm{~d}, J=11.7 \mathrm{~Hz}, 1 \mathrm{H})$, $6.11(\mathrm{~d}, J=11.7,1 \mathrm{H}), 4.56(\mathrm{q}, J=7.2 \mathrm{~Hz}, 1 \mathrm{H}), 3.13(\mathrm{dd}, J=13.9,4.2 \mathrm{~Hz}, 1 \mathrm{H})$, $2.54-2.47(\mathrm{~m}, 1 \mathrm{H}), 2.36-2.29(\mathrm{~m}, 2 \mathrm{H}), 2.12-2.05(\mathrm{~m}, 2 \mathrm{H}), 1.98-1.92(\mathrm{~m}$, 1H), $1.82-1.67(\mathrm{~m}, 5 \mathrm{H}), 1.54(\mathrm{~s}, 9 \mathrm{H}) .{ }^{13} \mathrm{C}$ NMR $\left(100 \mathrm{MHz}, \mathrm{CDCl}_{3}\right) \delta 220.0$, 162.9, 141.2, 135.6, 133.3, 131.2, 129.7, 129.3, 84.0, 64.9, 50.9, 38.1, 35.3, 29.3, 28.0, 20.5, 13.5.IR v 2980, 2935, 1731, 1349, 1313, 1251, 1152, 1126, $780,721 \mathrm{~cm}^{-1}$; HRMS (ESI) m/z: [M + Na] $]^{+}$Calcd. for $\mathrm{C}_{21} \mathrm{H}_{28} \mathrm{O}_{5} \mathrm{NaS} 415.1555$; found 415.1542 .

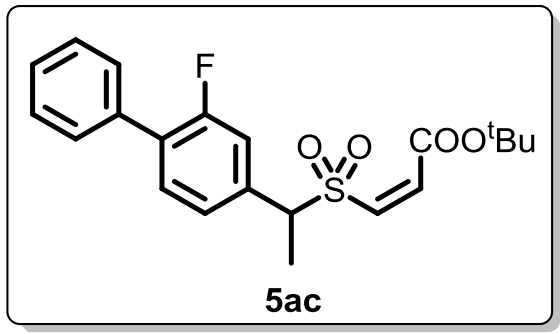

tert-butyl (Z)-3-((1-(2-fluoro-[1,1'-biphenyl] -4-yl)ethyl)sulfonyl)acrylate (5ac). Prepared following general procedure III using redox-active ester derived from (R)-2-Flurbiprofen (77.0mg, $0.2 \mathrm{mmol}$ ), $\mathrm{Na}_{2} \mathrm{~S}_{2} \mathrm{O}_{4}$ (69.6 mg, $\left.0.4 \mathrm{mmol}\right)$, tert-butyl proplolate ( $\left.50.5 \mathrm{mg}, 0.4 \mathrm{mmol}\right)$, DIPEA (51.7 mg, $0.4 \mathrm{mmol})$, TBAB (97.6 mg, $0.3 \mathrm{mmol})$, solvent $\left(\mathrm{DMF} / \mathrm{H}_{2} \mathrm{O}=9: 1,2\right.$ 
$\mathrm{mL}$ ), the reaction was stirred at $40{ }^{\circ} \mathrm{C}$ oil bath for $15 \mathrm{~h}$ giving $5 \mathrm{ac}$ in $59 \%$ yield $(45.8 \mathrm{mg})$ as an oil by column chromatography (PE/EA $\left.=2: 1, \mathrm{R}_{\mathrm{f}}=0.2\right) .{ }^{1} \mathrm{H}$ NMR $\left(400 \mathrm{MHz}, \mathrm{CDCl}_{3}\right) \delta 7.48-7.45(\mathrm{~m}, 2 \mathrm{H}), 7.40-7.35(\mathrm{~m}, 3 \mathrm{H}), 7.33-7.30$ (m, $1 \mathrm{H}), 7.24-7.18(\mathrm{~m}, 2 \mathrm{H}), 6.45(\mathrm{~d}, J=11.6 \mathrm{~Hz}, 1 \mathrm{H}), 6.16(\mathrm{~d}, J=11.6 \mathrm{~Hz}$, 1H), 4.61 (q, $J=7.2 \mathrm{~Hz}, 1 \mathrm{H}), 1.73(\mathrm{~d}, J=7.2 \mathrm{~Hz}, 3 \mathrm{H}), 1.50$ (s, 9H). ${ }^{13} \mathrm{C}$ NMR $\left(100 \mathrm{MHz}, \mathrm{CDCl}_{3}\right) \delta 162.8,159.6(\mathrm{~d}, J=248 \mathrm{~Hz}), 136.2,135.0,134.7(\mathrm{~d}, J=7$ $\mathrm{Hz}$ ), 133.4, 131.0 (d, $J=4 \mathrm{~Hz}$ ), 129.8 (d, $J=4 \mathrm{~Hz}), 128.9$ (d, $J=3 \mathrm{~Hz}), 128.5$, 128.0, $125.6(\mathrm{~d}, J=3 \mathrm{~Hz}), 117.2(\mathrm{~d}, J=24 \mathrm{~Hz}), 84.2,64.6,28.0,13.6 .{ }^{19} \mathrm{~F}$ NMR $\left(376 \mathrm{MHz}, \mathrm{CDCl}_{3}\right) \delta-116.65$. IR $\vee 2982,1729,1485,1369,1315,1253,1152$, 1128, 841, 726, $699 \mathrm{~cm}^{-1}$; HRMS (ESI) m/z: $\left[\mathrm{M}+\mathrm{Na}^{+}\right.$Calcd. for $\mathrm{C}_{21} \mathrm{H}_{23} \mathrm{O}_{4} \mathrm{FNaS}$ 413.1199; found 413.1185 .

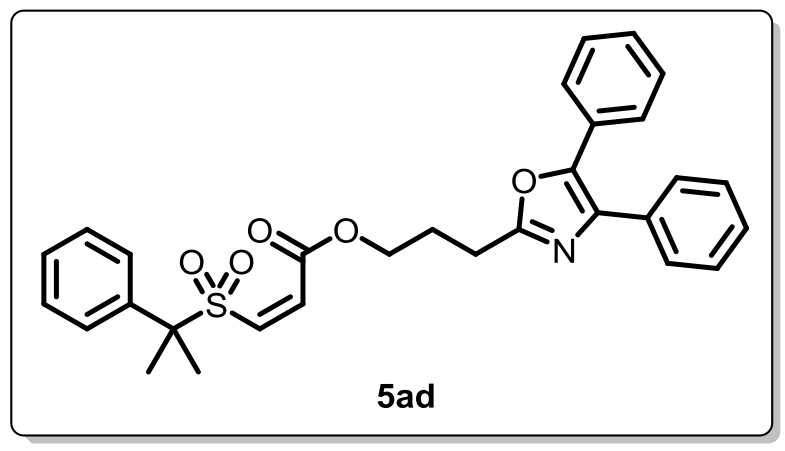

$\begin{array}{lr}\text { 3-(4,5-diphenyloxazol-2-yl)pro } \\ \text { pyl (Z)-3-((2-phenylpropan-2-yl) } \\ \text { sulfonyl)acrylate r } & \text { (5ad). } \\ \text { Prepared following } & \text { general } \\ \text { procedure III } & \text { using } \\ \text { 1,3-dioxoisoindolin-2-yl } & \text { 2-methyl- }\end{array}$

2- phenyl propanoate $(61.9 \mathrm{mg}, 0.2 \mathrm{mmol}), \mathrm{Na}_{2} \mathrm{~S}_{2} \mathrm{O}_{4}(69.6 \mathrm{mg}, 0.4 \mathrm{mmol})$, alkyne michael acceptor derived from Oxaprozin (133.0 mg, $0.4 \mathrm{mmol}$ ), DIPEA (51.7 mg, $0.4 \mathrm{mmol})$, TBAB (97.6 mg, $0.3 \mathrm{mmol}$ ), solvent (DMF/ $\mathrm{H}_{2} \mathrm{O}=9: 1,2$ $\mathrm{mL}$ ), the reaction was stirred at $40{ }^{\circ} \mathrm{C}$ oil bath for $15 \mathrm{~h}$ giving $5 \mathrm{ad}$ in $61 \%$ yield $(63.0 \mathrm{mg})$ as an oil by column chromatography (PE/EA $\left.=2: 1, R_{f}=0.3\right) .{ }^{1} \mathbf{H}$ NMR (400 MHz, $\left.\mathrm{CDCl}_{3}\right) \delta 7.67-7.62(\mathrm{~m}, 2 \mathrm{H}), 7.61-7.55(\mathrm{~m}, 4 \mathrm{H}), 7.41-7.30$ (m, 9H), $6.54(\mathrm{~d}, J=11.7 \mathrm{~Hz}, 1 \mathrm{H}), 5.97(\mathrm{~d}, J=11.7 \mathrm{~Hz}, 1 \mathrm{H}), 4.41$ (t, $J=6.2 \mathrm{~Hz}$, 2H), 3.00 (t, $J=7.4 \mathrm{~Hz}, 2 \mathrm{H}), 2.33-2.25(\mathrm{~m}, 2 \mathrm{H}), 1.85$ (s, 6H). ${ }^{13} \mathrm{C}$ NMR (100 $\left.\mathrm{MHz}, \mathrm{CDCl}_{3}\right) \delta 164.1,162.4,145.3,136.5,135.0,134.4,132.4,128.9,128.7$, 128.6, 128.5, 128.4, 128.3, 128.0, 127.8, 126.4, 64.9, 64.8, 25.8, 24.8, 22.2.IR v 2854, 1737, 1711, 1362, 1156, 1120, 1097, 775, 765, $694 \mathrm{~cm}^{-1}$; HRMS (ESI) $\mathrm{m} / \mathrm{z}:[\mathrm{M}+\mathrm{H}]^{+}$Calcd. for $\mathrm{C}_{30} \mathrm{H}_{30} \mathrm{NO}_{5} \mathrm{~S}$ 516.1845; found 516.1839. 


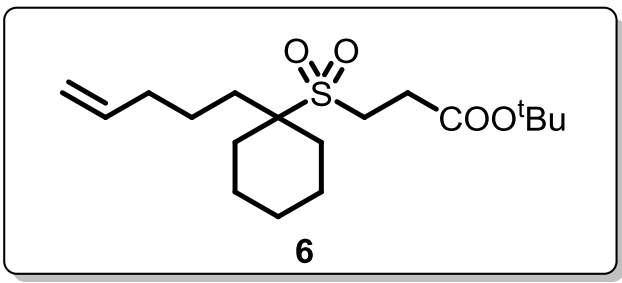

tert-butyl

cyclohexyl)sulfonyl)propanoate

(6).

Prepared following general procedure II using 1,3-dioxoisoindolin-2-yl 1-(pent-4-

en-1-yl)cyclohexane-1-carboxylate $(68.3 \mathrm{mg}, 0.2 \mathrm{mmol}), \mathrm{Na}_{2} \mathrm{~S}_{2} \mathrm{O}_{4}(52.2 \mathrm{mg}$, $0.3 \mathrm{mmol})$, tert-Butyl acrylate $(76.9 \mathrm{mg}, 0.6 \mathrm{mmol}), \mathrm{Et}_{3} \mathrm{~N} . \mathrm{HCl}(68.8 \mathrm{mg}, 0.5$ $\mathrm{mmol})$, LiOAc (13.2 $\mathrm{mg}, 0.2 \mathrm{mmol})$ and DMF $(2 \mathrm{~mL})$, the reaction was stirred at $100{ }^{\circ} \mathrm{C}$ oil bath for $15 \mathrm{~h}$ giving 6 in $28 \%$ yield $(19.6 \mathrm{mg}$ ) as an oil by column chromatography $\left(\mathrm{PE} / \mathrm{EA}=5: 1, \mathrm{R}_{\mathrm{f}}=0.3\right) .{ }^{1} \mathrm{H}$ NMR $\left(400 \mathrm{MHz}, \mathrm{CDCl}_{3}\right) \delta 5.84-$ $5.74(m, 1 H), 5.07-4.97(m, 2 H), 3.20-3.12(m, 2 H), 2.83-2.75(m, 2 H)$, $2.10-2.04(\mathrm{~m}, 2 \mathrm{H}), 1.88-1.80(\mathrm{~m}, 6 \mathrm{H}), 1.77-1.71(\mathrm{~m}, 2 \mathrm{H}), 1.68-1.62(\mathrm{~m}$, 2H), $1.46(\mathrm{~s}, 9 \mathrm{H}), 1.42-1.35(\mathrm{~m}, 2 \mathrm{H}), 1.27-1.19(\mathrm{~m}, 2 \mathrm{H}) .{ }^{13} \mathrm{C}$ NMR $(100 \mathrm{MHz}$, $\left.\mathrm{CDCl}_{3}\right) \delta 170.2,138.0,115.3,81.7,65.4,41.9,34.2,29.4,28.2,28.0,26.6$, 24.8, 22.6, 21.3.IR $\vee 2942,2860,1731,1462,1368,1301,1126,909,848 \mathrm{~cm}^{-1}$; HRMS (ESI) m/z: $[\mathrm{M}+\mathrm{Na}]^{+}$Calcd. for $\mathrm{C}_{18} \mathrm{H}_{32} \mathrm{O}_{4} \mathrm{NaS}$ 367.1919; found 367.1908.

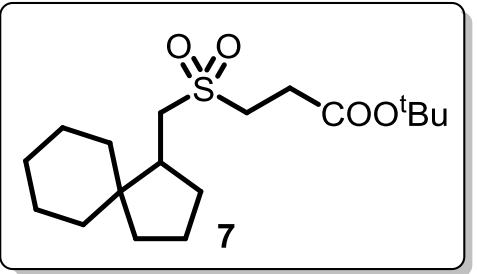

tert-butyl 3-((spiro[4.5]decan-1-ylmethyl) sulfonyl)propanoate (7). Prepared following general procedure II using 1,3-dioxoisoindolin-2-yl 1-(pent-4-en-1-yl) cyclohexane-1-carboxylate (68.3 mg, $0.2 \mathrm{mmol}), \mathrm{Na}_{2} \mathrm{~S}_{2} \mathrm{O}_{4}(52.2 \mathrm{mg}, 0.3 \mathrm{mmol})$, tert-Butyl acrylate (76.9 $\mathrm{mg}, 0.6 \mathrm{mmol}), \mathrm{Et}_{3} \mathrm{~N} . \mathrm{HCl}(68.8 \mathrm{mg}, 0.5 \mathrm{mmol}), \mathrm{LiOAc}(13.2 \mathrm{mg}, 0.2 \mathrm{mmol})$ and DMF (2 mL), the reaction was stirred at $100{ }^{\circ} \mathrm{C}$ oil bath for $15 \mathrm{~h}$ giving 7 in $44 \%$ yield $(30.5 \mathrm{mg})$ as an oil by column chromatography $\left(\mathrm{PE} / \mathrm{EA}=5: 1, \mathrm{R}_{\mathrm{f}}=0.3\right) .{ }^{1} \mathrm{H}$ NMR $\left(400 \mathrm{MHz}, \mathrm{CDCl}_{3}\right) \delta 3.26(\mathrm{t}, J=7.5 \mathrm{~Hz}, 2 \mathrm{H}), 3.08$ (dd, $J=13.6,1.8 \mathrm{~Hz}$, $1 \mathrm{H}), 2.83-2.75(\mathrm{~m}, 3 \mathrm{H}), 2.23-2.14(\mathrm{~m}, 1 \mathrm{H}), 2.00-1.90(\mathrm{~m}, 1 \mathrm{H}), 1.85-1.78$ $(\mathrm{m}, 1 \mathrm{H}), 1.69-1.50(\mathrm{~m}, 6 \mathrm{H}), 1.46(\mathrm{~s}, 9 \mathrm{H}), 1.37-1.24(\mathrm{~m}, 6 \mathrm{H}), 1.14-1.02(\mathrm{~m}$, 1H), $0.91-0.83(\mathrm{~m}, 1 \mathrm{H}) .{ }^{13} \mathrm{C}$ NMR $\left(100 \mathrm{MHz}, \mathrm{CDCl}_{3}\right) \delta 169.6,81.9,54.9,49.1$, $45.4,43.5,37.0,34.1,30.1,29.6,28.0,26.4,23.9,22.3,21.6$. IR $\vee 2928,1731$, 
1368, 1312, 1158, 1125, $844 \mathrm{~cm}^{-1}$; HRMS (ESI) m/z: $\left[\mathrm{M}+\mathrm{Na}^{+}\right.$Calcd. for $\mathrm{C}_{18} \mathrm{H}_{32} \mathrm{O}_{4} \mathrm{NaS} 367.1919$; found 367.1909.

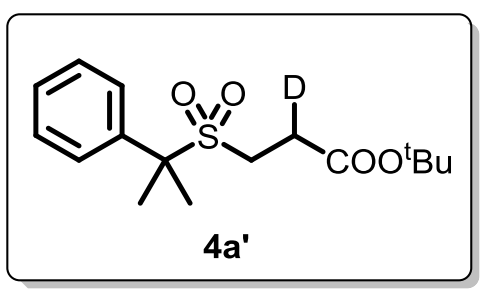

tert-butyl 3-((2-phenylpropan-2-yl)sulfonyl) propanoate-2-d (4a'). Prepared following general procedure II using 1,3-dioxoisoindolin- 2-yl 2methyl-2- phenyl propanoate $(61.9 \mathrm{mg}, 0.2 \mathrm{mmol})$,

$\mathrm{Na}_{2} \mathrm{~S}_{2} \mathrm{O}_{4}(52.2 \mathrm{mg}, 0.3 \mathrm{mmol})$, tert-Butyl acrylate $(76.9 \mathrm{mg}, 0.6 \mathrm{mmol})$, $\mathrm{Et}_{3} \mathrm{~N} . \mathrm{DCl}(69.3 \mathrm{mg}, 0.5 \mathrm{mmol})$, LiOAc (13.2 mg, $\left.0.2 \mathrm{mmol}\right)$ and DMF (2 mL), the reaction was stirred at $100{ }^{\circ} \mathrm{C}$ oil bath for $15 \mathrm{~h}$ giving $4 \mathrm{a}^{\prime}$ in $64 \%$ yield $(39.9$ $\mathrm{mg}$ ) as a white solid by column chromatography (PE/EA $\left.=5: 1, \mathrm{R}_{\mathrm{f}}=0.3\right) .{ }^{1} \mathrm{H}$ NMR $\left(400 \mathrm{MHz}, \mathrm{CDCl}_{3}\right) \delta 7.68-7.58(\mathrm{~m}, 2 \mathrm{H}), 7.42-7.36(\mathrm{~m}, 3 \mathrm{H}), 2.87$ (d, $J=$ $7.8 \mathrm{~Hz}, 2 \mathrm{H}), 2.55-2.49(\mathrm{~m}, 1 \mathrm{H}), 1.86(\mathrm{~s}, 6 \mathrm{H}), 1.39(\mathrm{~s}, 9 \mathrm{H}) .{ }^{13} \mathrm{C} \mathrm{NMR}(100 \mathrm{MHz}$, $\left.\mathrm{CDCl}_{3}\right) \delta 169.8,137.1,128.7,128.6,128.0,81.6,64.9,41.9,27.9,26.54(\mathrm{t}, J=$ $20.0 \mathrm{~Hz}$ ), 22.4. IR $\vee 2979,2932,1726,1369,1297,1149,1119,1097,776,698$ $\mathrm{cm}^{-1}$; HRMS (ESI) m/z: $[\mathrm{M}+\mathrm{H}]^{+}$Calcd. for $\mathrm{C}_{16} \mathrm{H}_{24} \mathrm{DO}_{4} \mathrm{~S}$ 314.1536; found 314.1532.

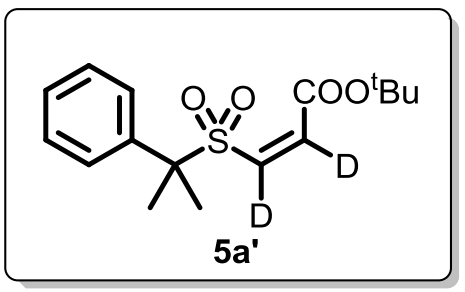

tert-butyl (Z)-3-((2-phenylpropan-2-yl)sulfonyl) acrylate-d2 (5a'). Prepared following general procedure III using 1,3-dioxoisoindolin- 2-yl 2methyl-2- phenyl propanoate $(61.9 \mathrm{mg}, 0.2 \mathrm{mmol})$, $\mathrm{Na}_{2} \mathrm{~S}_{2} \mathrm{O}_{4}(69.6 \mathrm{mg}, 0.4 \mathrm{mmol})$, tert-butyl proplolate (50.5 mg, $0.4 \mathrm{mmol})$, DIPEA (51.7 mg, $0.4 \mathrm{mmol})$, TBAB (97.6 mg, $0.3 \mathrm{mmol})$, solvent $\left(\mathrm{DMF} / \mathrm{D}_{2} \mathrm{O}=9: 1,2 \mathrm{~mL}\right)$, the reaction was stirred at $40{ }^{\circ} \mathrm{C}$ oil bath for 15 h giving $5 a^{\prime}$ in $86 \%$ yield $(53.7 \mathrm{mg}, \mathrm{Z} / \mathrm{E}=7: 1)$ as a white solid by column chromatography $\left(\mathrm{PE} / \mathrm{EA}=5: 1, \mathrm{R}_{\mathrm{f}}=0.3\right) .{ }^{1} \mathrm{H}$ NMR $\left(400 \mathrm{MHz}, \mathrm{CDCl}_{3}\right) \delta 7.61-$ $7.59(\mathrm{~m}, 2 \mathrm{H}), 7.43-7.32(\mathrm{~m}, 3 \mathrm{H}), 1.85(\mathrm{~s}, 6 \mathrm{H}), 1.54(\mathrm{~s}, 9 \mathrm{H}) .{ }^{13} \mathrm{C}$ NMR (100 $\left.\mathrm{MHz}, \mathrm{CDCl}_{3}\right) \delta 162.9,136.8,135.43,135.2(\mathrm{t}, J=10.0 \mathrm{~Hz}), 128.6,128.5$, 128.3, $126.5(\mathrm{t}, J=14.0 \mathrm{~Hz}), 83.7,64.9,27.9,22.2 . I R \vee 2983,2931,1734$, 
1369, 1306, 1164, 1128, 846, 775, $699 \mathrm{~cm}^{-1}$; HRMS (ESI) m/z: $[\mathrm{M}+\mathrm{Na}]^{+}$Calcd. for $\mathrm{C}_{16} \mathrm{H}_{20} \mathrm{D}_{2} \mathrm{O}_{4} \mathrm{SNa} 335.1262$; found 335.1249 .

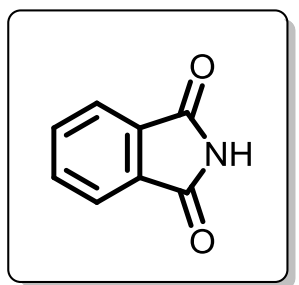

isoindoline-1,3-dione. ${ }^{1} \mathbf{H}$ NMR (400 MHz, $d_{6}$-DMSO) $\delta$ $11.33(\mathrm{~s}, 1 \mathrm{H}), 7.82(\mathrm{~s}, 4 \mathrm{H}) .{ }^{13} \mathrm{C}$ NMR $\left(100 \mathrm{MHz}, \mathrm{d}_{6}\right.$-DMSO) $\delta$ 169.3, 134.4, 132.7, 123. 


\section{X-Ray Crystal Structures}

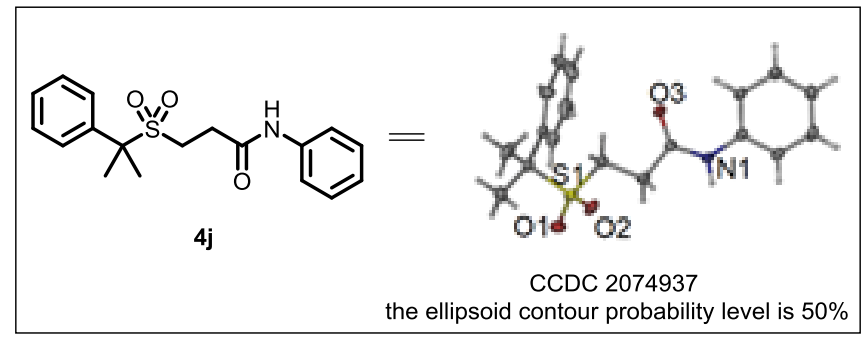

Note: The single crystal was obtained by slow volatilization in the mixed solvent of ethyl acetate and dichloromethane.

Table S2. Sample and crystal data for $\mathbf{4 j}$ (CCDC 2074937, the ellipsoid contour probability level is $50 \%$ ).

\begin{tabular}{|c|c|c|}
\hline \multirow[t]{2}{*}{ Bond precision } & \multicolumn{2}{|l|}{$C-C=0.0023 \mathrm{~A}$} \\
\hline & \multicolumn{2}{|c|}{ Wavelength $=1.54184$} \\
\hline \multirow[t]{3}{*}{ Cell } & $\mathrm{a}=6.8147(2)$ & $\alpha=88.915$ \\
\hline & $b=10.8499$ & $\beta=82.601$ \\
\hline & $\mathrm{C}=11.5170(4)$ & $Y=81.476(2)$ \\
\hline Temperature & \multicolumn{2}{|l|}{$170 \mathrm{~K}$} \\
\hline Volume & \multicolumn{2}{|l|}{$835.14(5)$} \\
\hline Space group & \multicolumn{2}{|l|}{$P-1$} \\
\hline Sum formula & \multicolumn{2}{|l|}{$\mathrm{C} 18 \mathrm{H} 21 \mathrm{NO} 3 \mathrm{~S}$} \\
\hline $\mathrm{Mr}$ & \multicolumn{2}{|l|}{331.42} \\
\hline $\mathrm{Dx}, \mathrm{g} \mathrm{cm}^{-3}$ & \multicolumn{2}{|l|}{1.318} \\
\hline Z & \multicolumn{2}{|l|}{2} \\
\hline $\mathrm{Mu}(\mathrm{mm}-1)$ & \multicolumn{2}{|l|}{1.841} \\
\hline F000 & \multicolumn{2}{|l|}{352.0} \\
\hline $\mathrm{h}, \mathrm{k}, \mathrm{Imax}$ & \multicolumn{2}{|l|}{$8,13,14$} \\
\hline Nref & \multicolumn{2}{|l|}{3250} \\
\hline Tmin,Tmax & \multicolumn{2}{|l|}{$0.514,1.000$} \\
\hline $\begin{array}{l}\text { Correction method= \# Reported } \\
\text { T Limits }\end{array}$ & \multirow[t]{2}{*}{$\operatorname{Tmin}=0.514$} & \multirow[t]{2}{*}{$\operatorname{Tmax}=1.000$} \\
\hline AbsCorr = MULTI-SCAN & & \\
\hline Data completeness & \multicolumn{2}{|l|}{0.954} \\
\hline Theta(max) & \multicolumn{2}{|l|}{74.232} \\
\hline $\mathrm{R}$ (reflections) & \multicolumn{2}{|l|}{$0.0400(3095)$} \\
\hline wR2(reflections) & \multicolumn{2}{|l|}{$0.1064(3250)$} \\
\hline $\mathrm{S}$ & \multicolumn{2}{|l|}{1.034} \\
\hline Npar & \multicolumn{2}{|l|}{210} \\
\hline
\end{tabular}




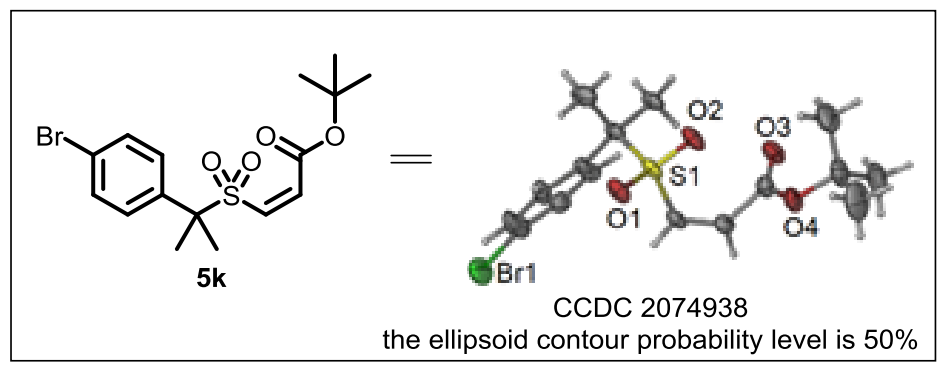

Note: The single crystal was obtained by slow volatilization in the mixed solvent of ethyl acetate and dichloromethane.

Table S3. Sample and crystal data for $\mathbf{5 k}$ (CCDC 2074938, the ellipsoid contour probability level is $50 \%$ ).

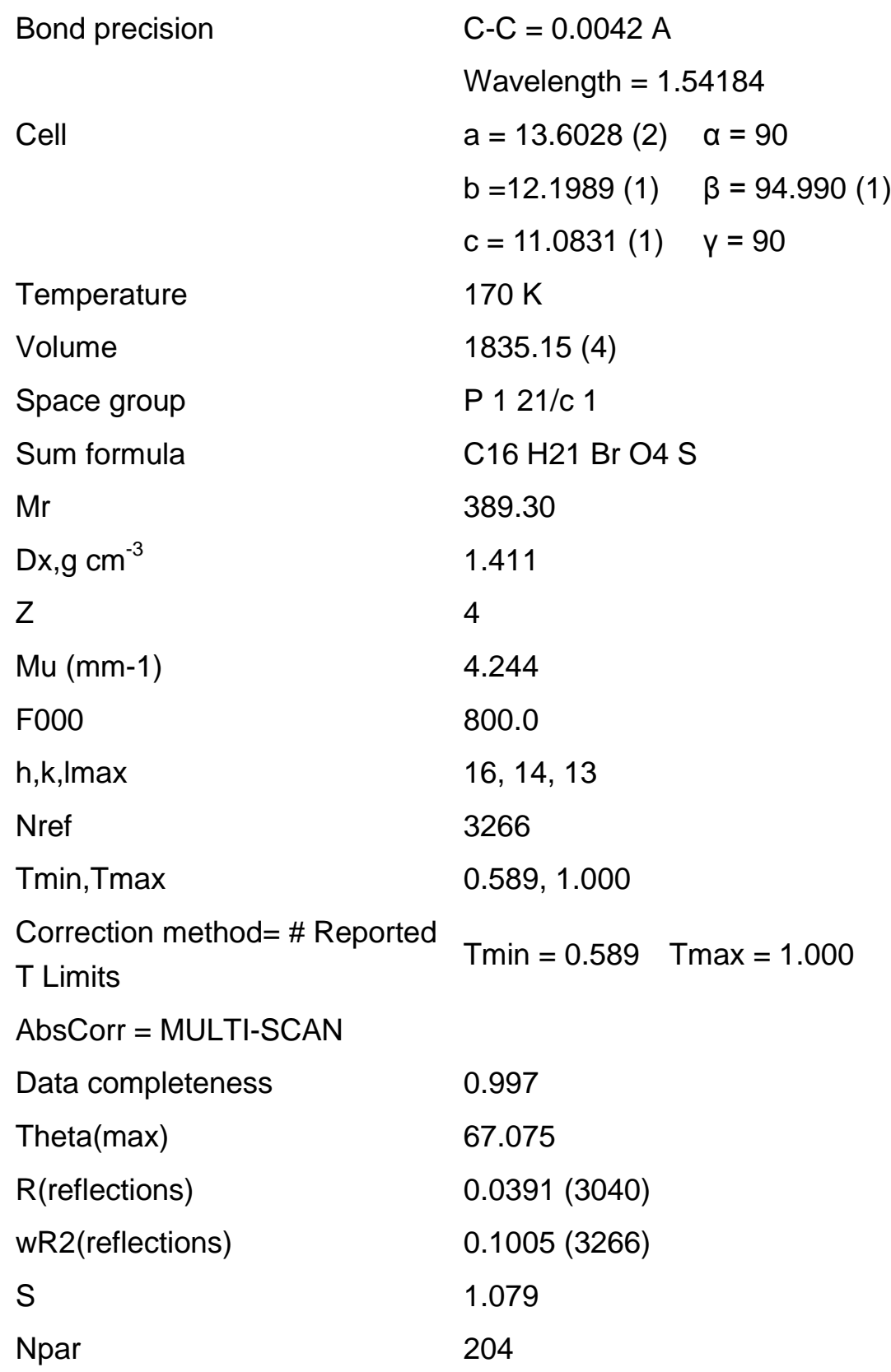


VII. The kinetic studies

(1) Rates for varying RCOOR'
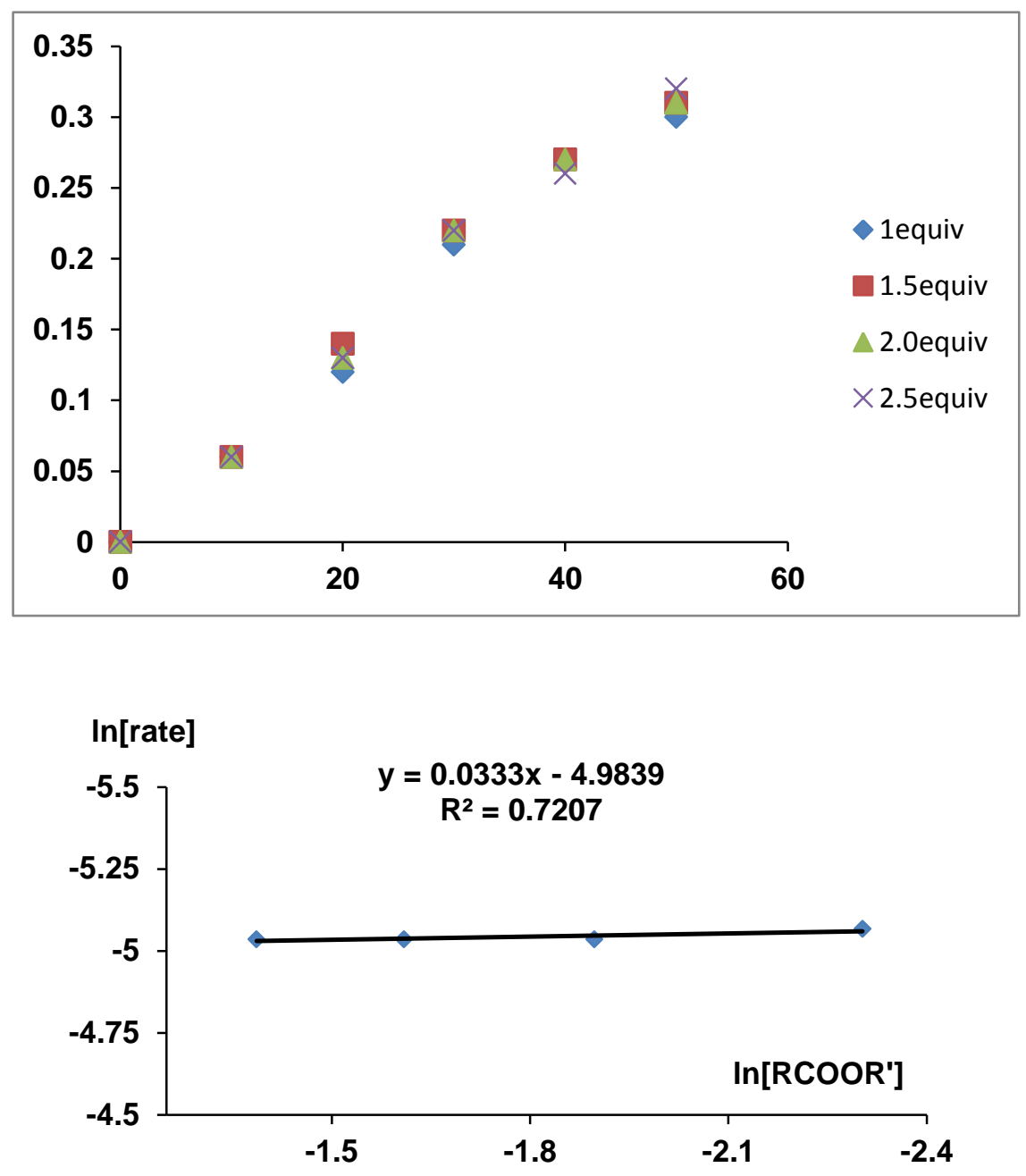
(2) Rates for varying $\mathrm{Et}_{3} \mathrm{~N} . \mathrm{HCl}$
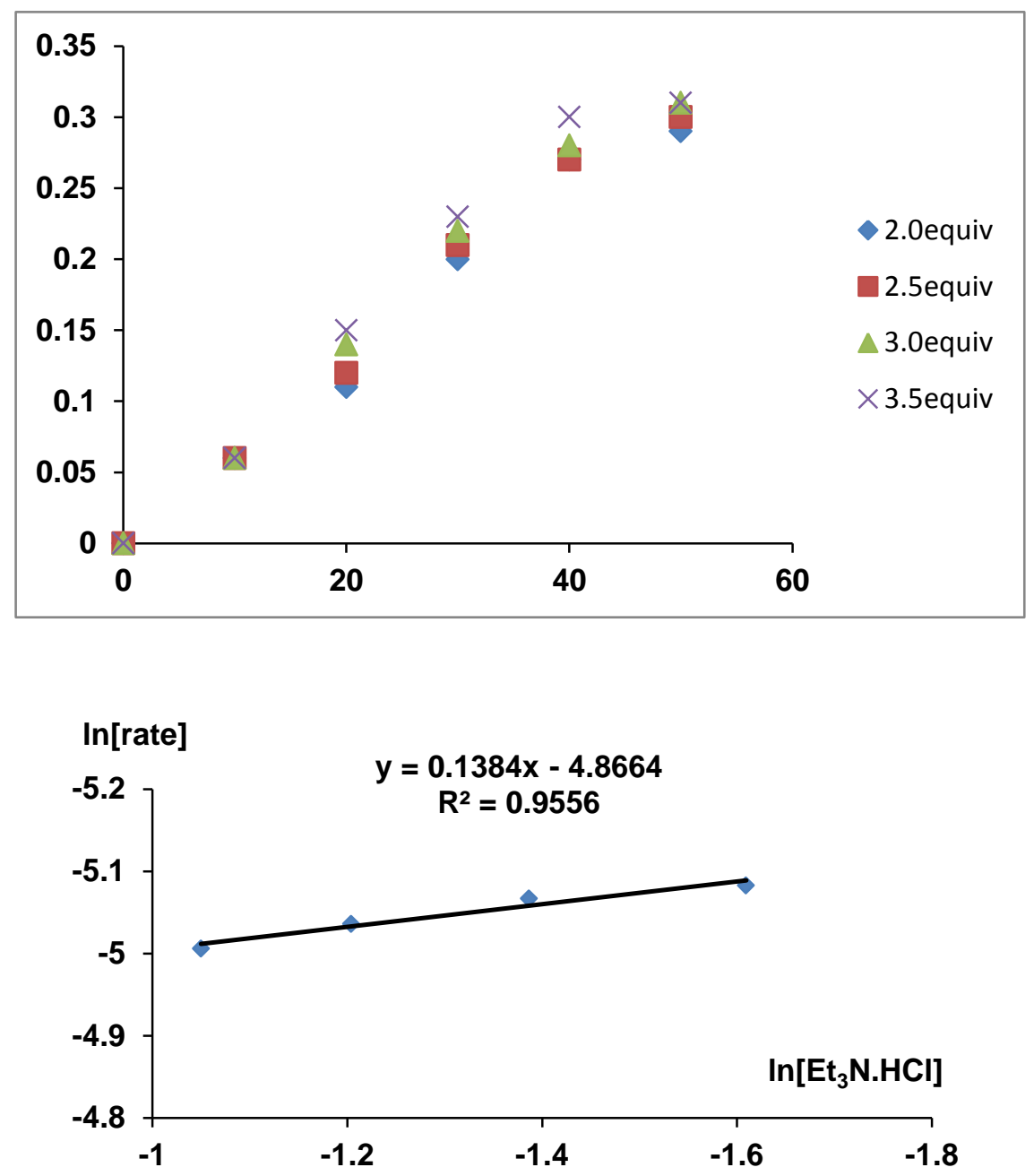
(3) Rates for varying Michael receptor
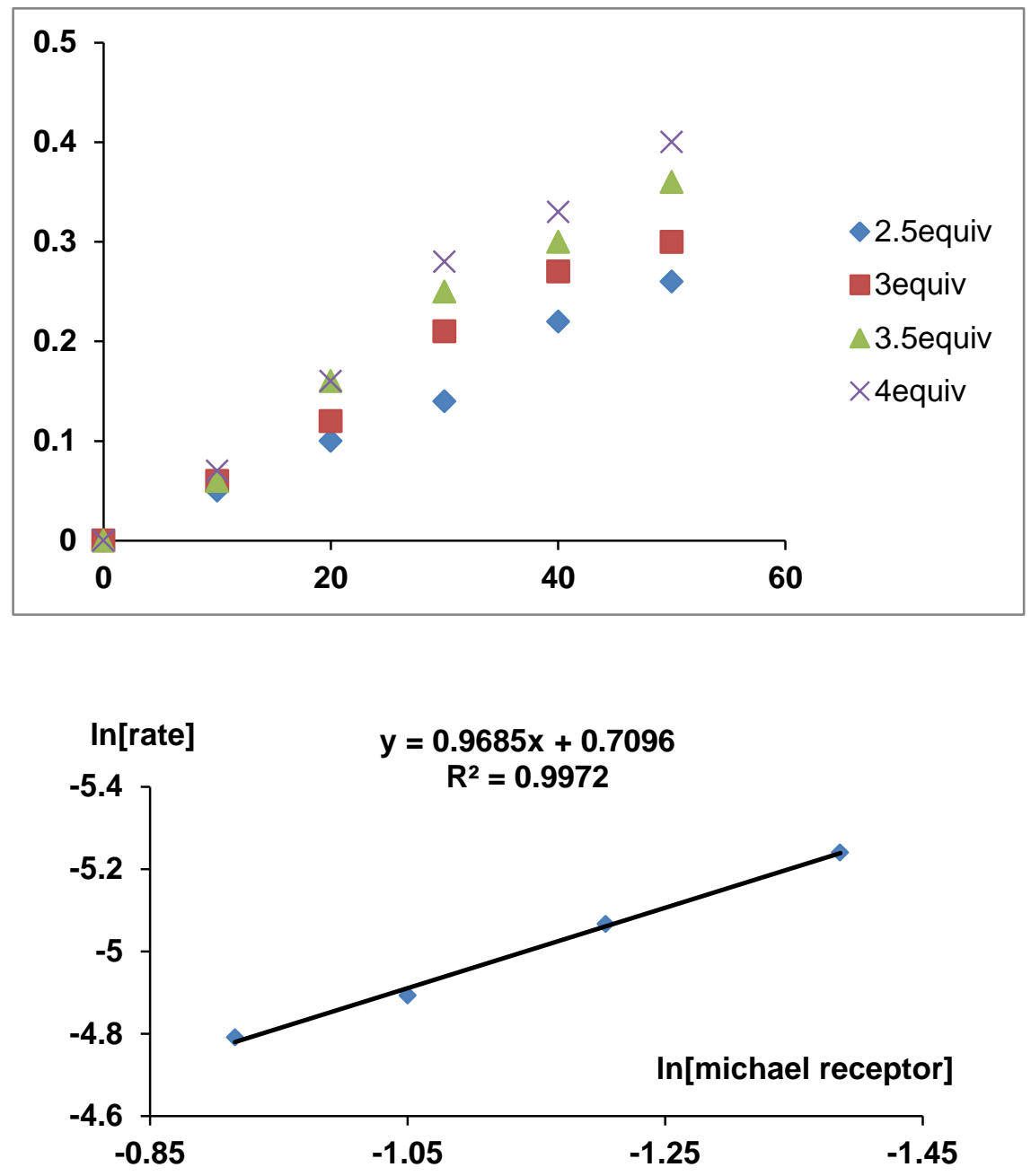
VIII. NMR spectra

${ }^{1} \mathbf{H}$ NMR $\left(400 \mathrm{MHz}, \mathrm{CDCl}_{3}\right)$

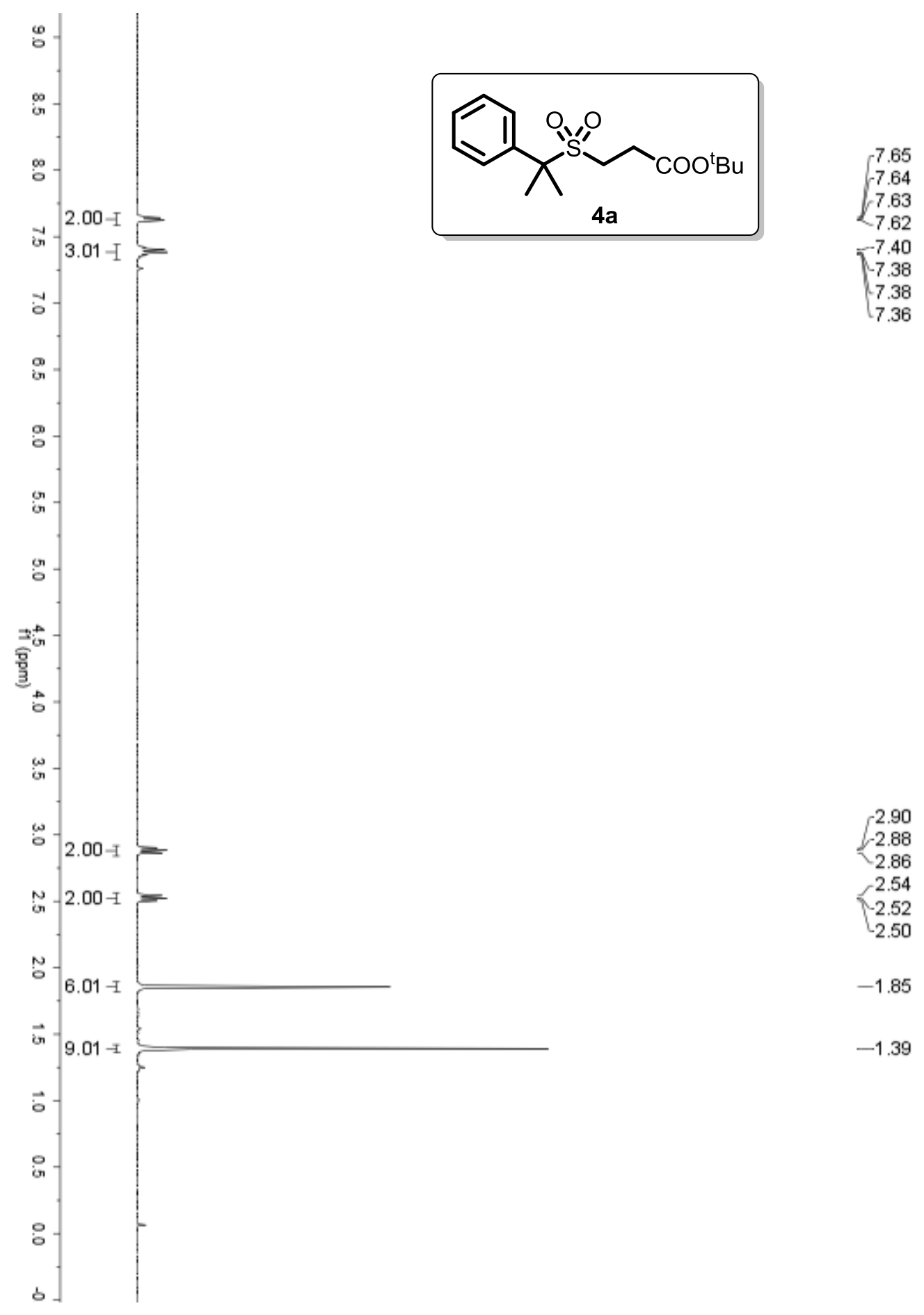


${ }^{13} \mathrm{C}$ NMR $\left(100 \mathrm{MHz}, \mathrm{CDCl}_{3}\right)$

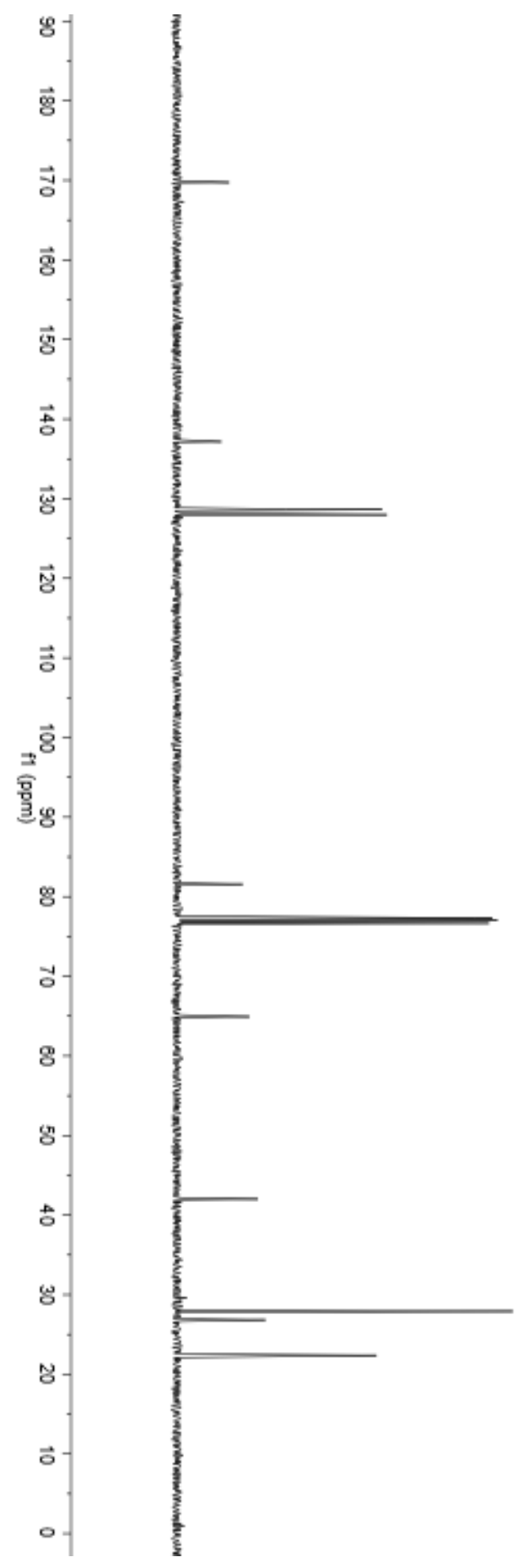

$-169.74$

$-137.16$

128.69

$-128.64$

128.00

$-81.58$

$-64.93$

$-42.01$

$-26.84$

$-22.39$ 
${ }^{1} \mathbf{H}$ NMR $\left(400 \mathrm{MHz}, \mathrm{CDCl}_{3}\right)$
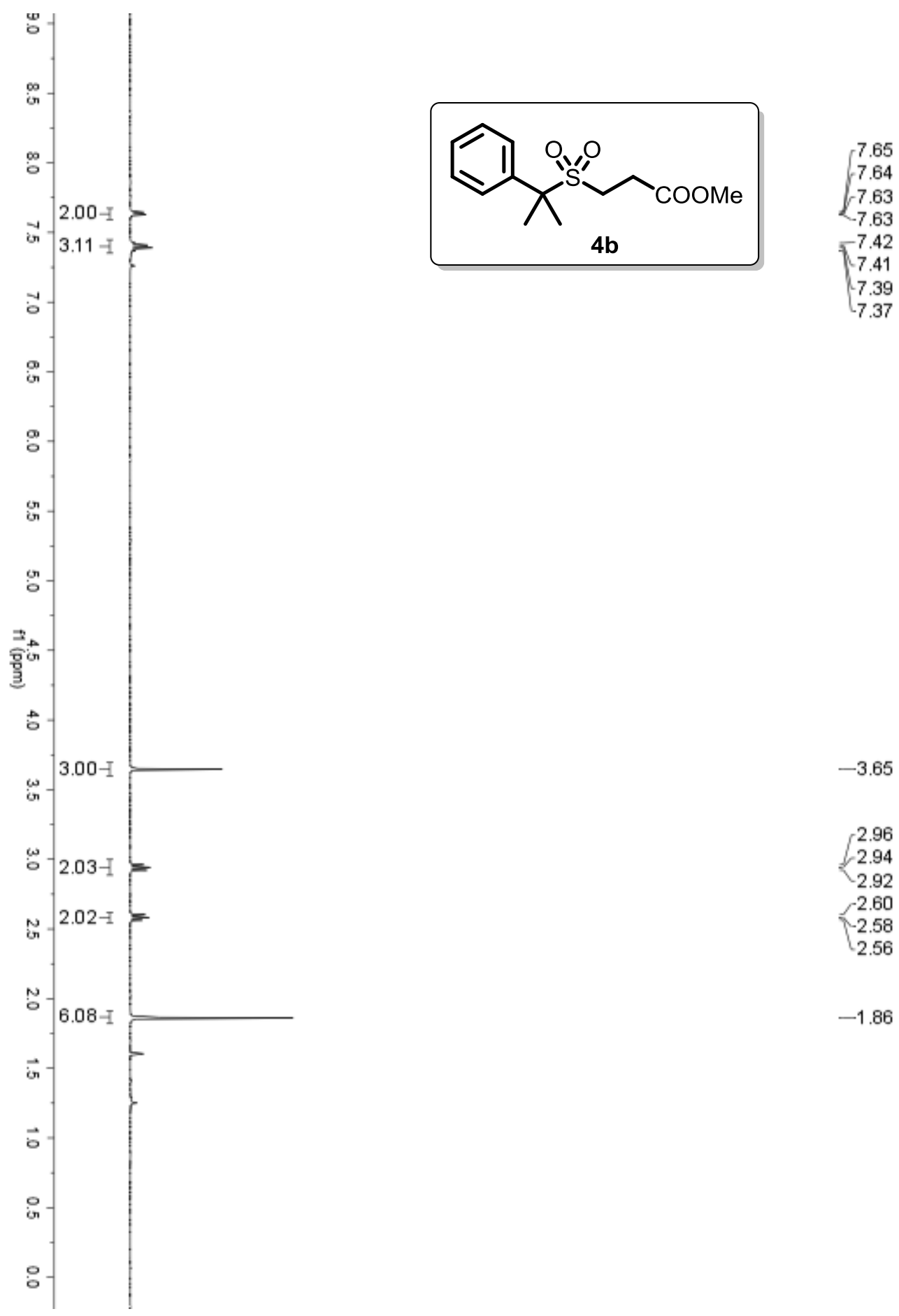
${ }^{13} \mathrm{C}$ NMR $\left(100 \mathrm{MHz}, \mathrm{CDCl}_{3}\right)$
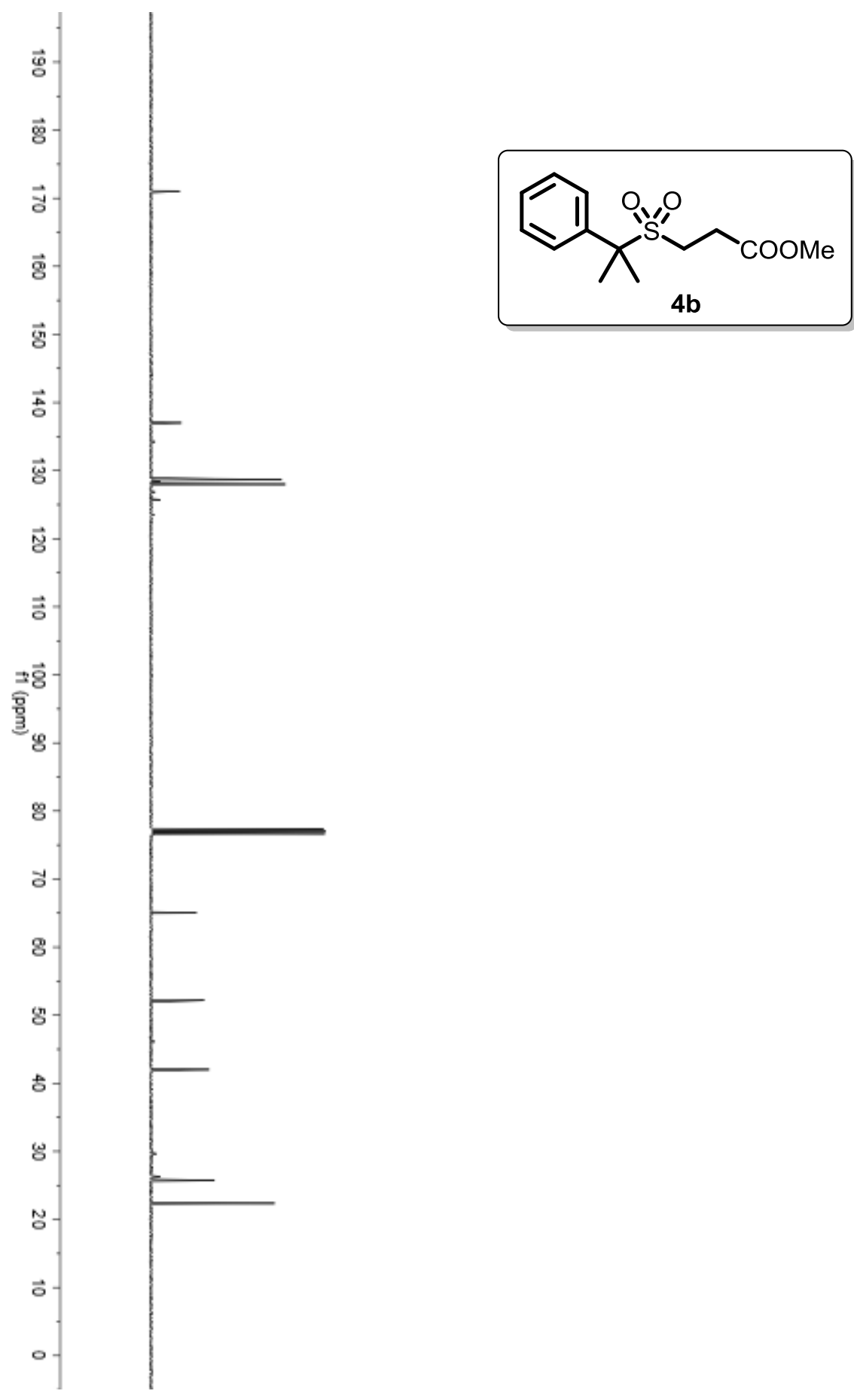

$-171.06$

$-137.03$

128.77

$-128.67$

128.00

$-65.04$

$-52.19$

$-42.03$

$-25.73$

$-22.38$ 
${ }^{1} \mathrm{H}$ NMR $\left(400 \mathrm{MHz}, \mathrm{CDCl}_{3}\right)$

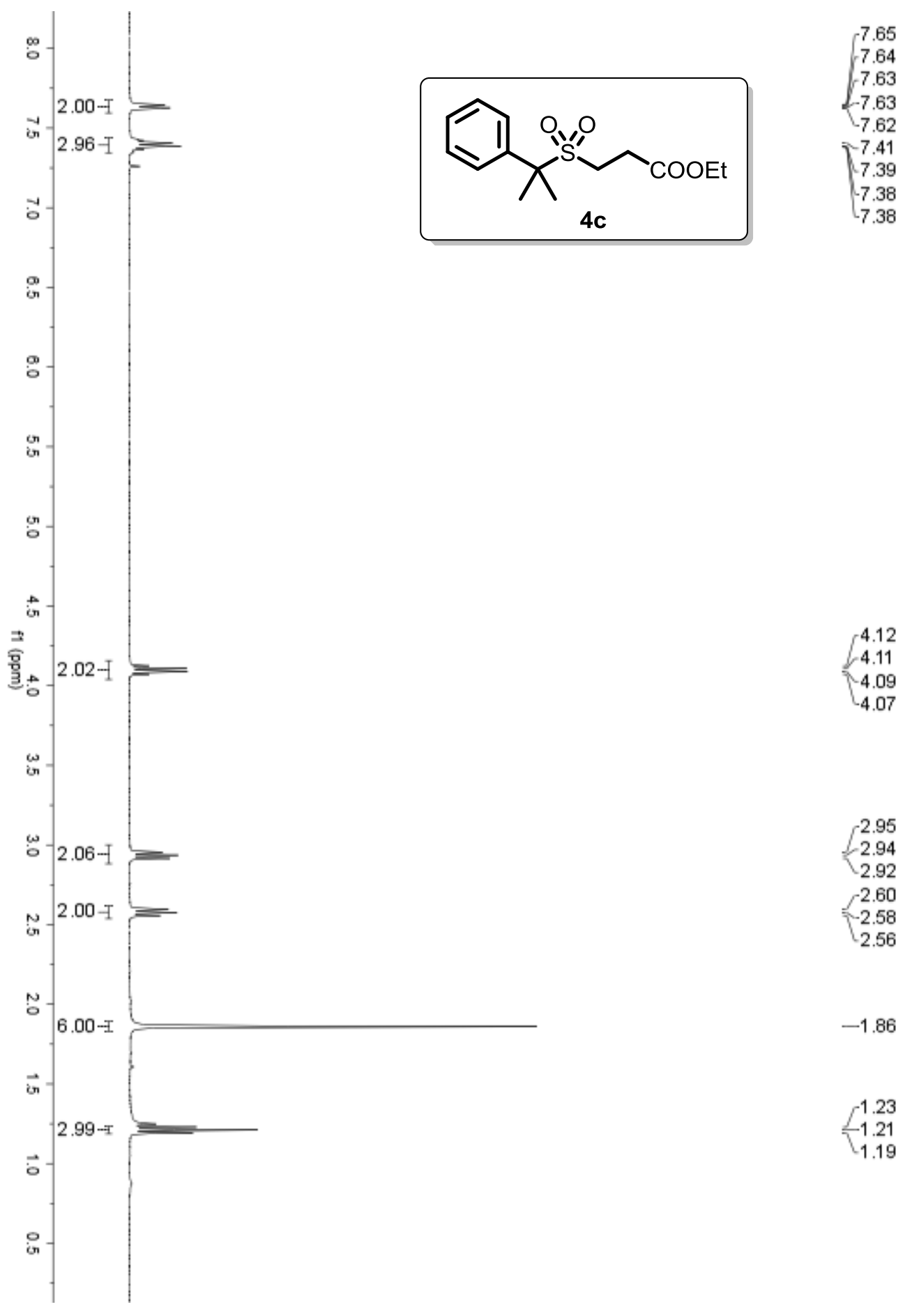


${ }^{13} \mathrm{C}$ NMR $\left(100 \mathrm{MHz}, \mathrm{CDCl}_{3}\right)$
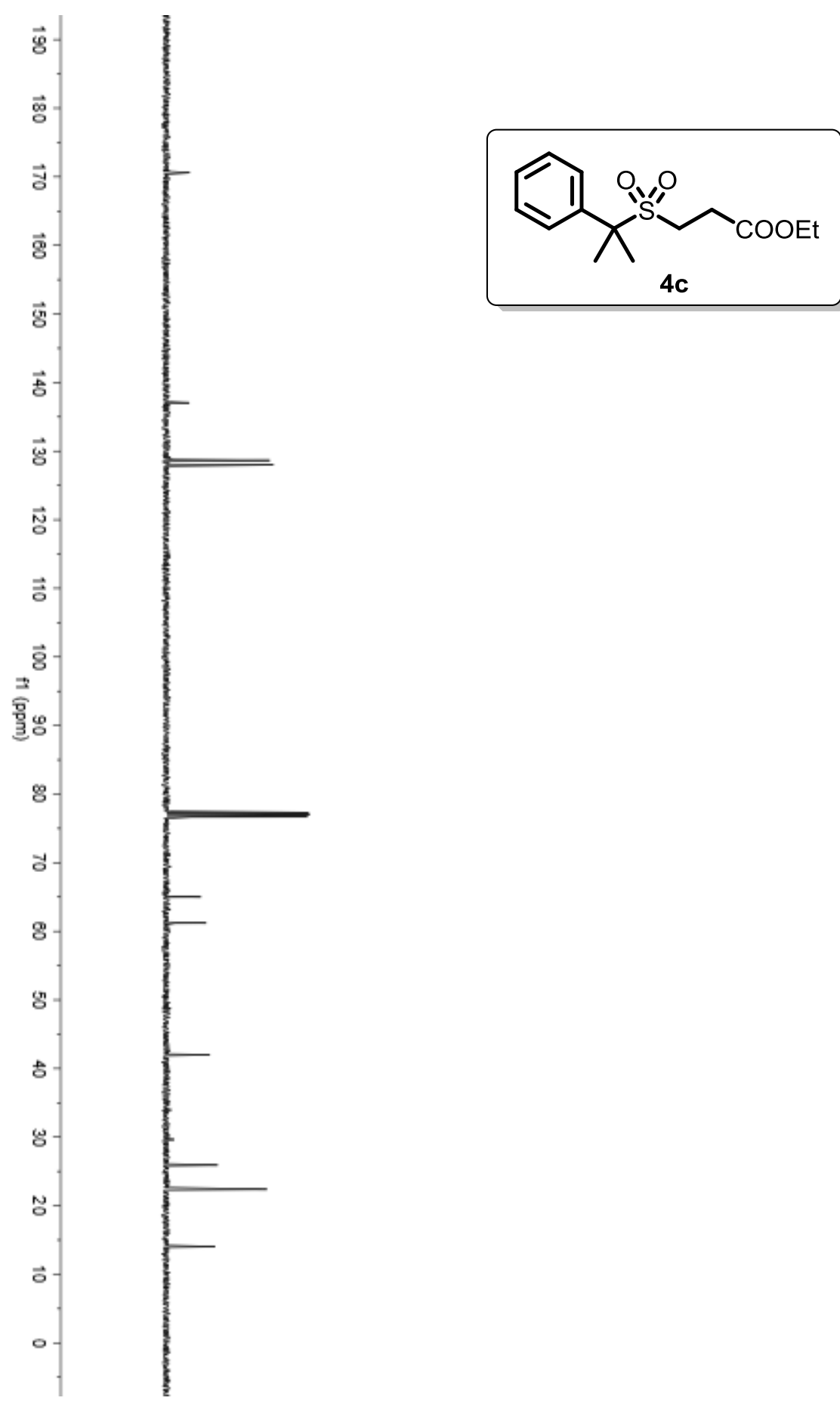

$-170.59$

$-137.05$

128.75

$-128.66$

$-128.00$

$-65.00$

$-61.20$

$-42.00$

$-25.91$

$-22.38$

$-14.03$ 
${ }^{1} \mathbf{H}$ NMR $\left(400 \mathrm{MHz}, \mathrm{CDCl}_{3}\right)$

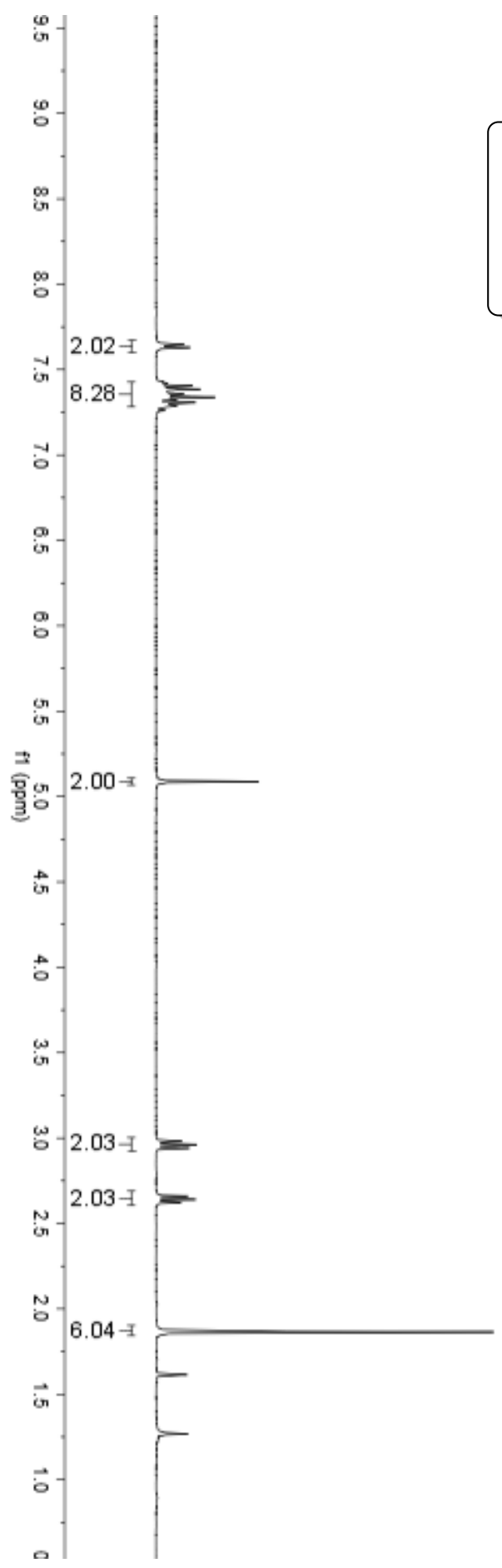


${ }^{13} \mathrm{C}$ NMR $\left(100 \mathrm{MHz}, \mathrm{CDCl}_{3}\right)$
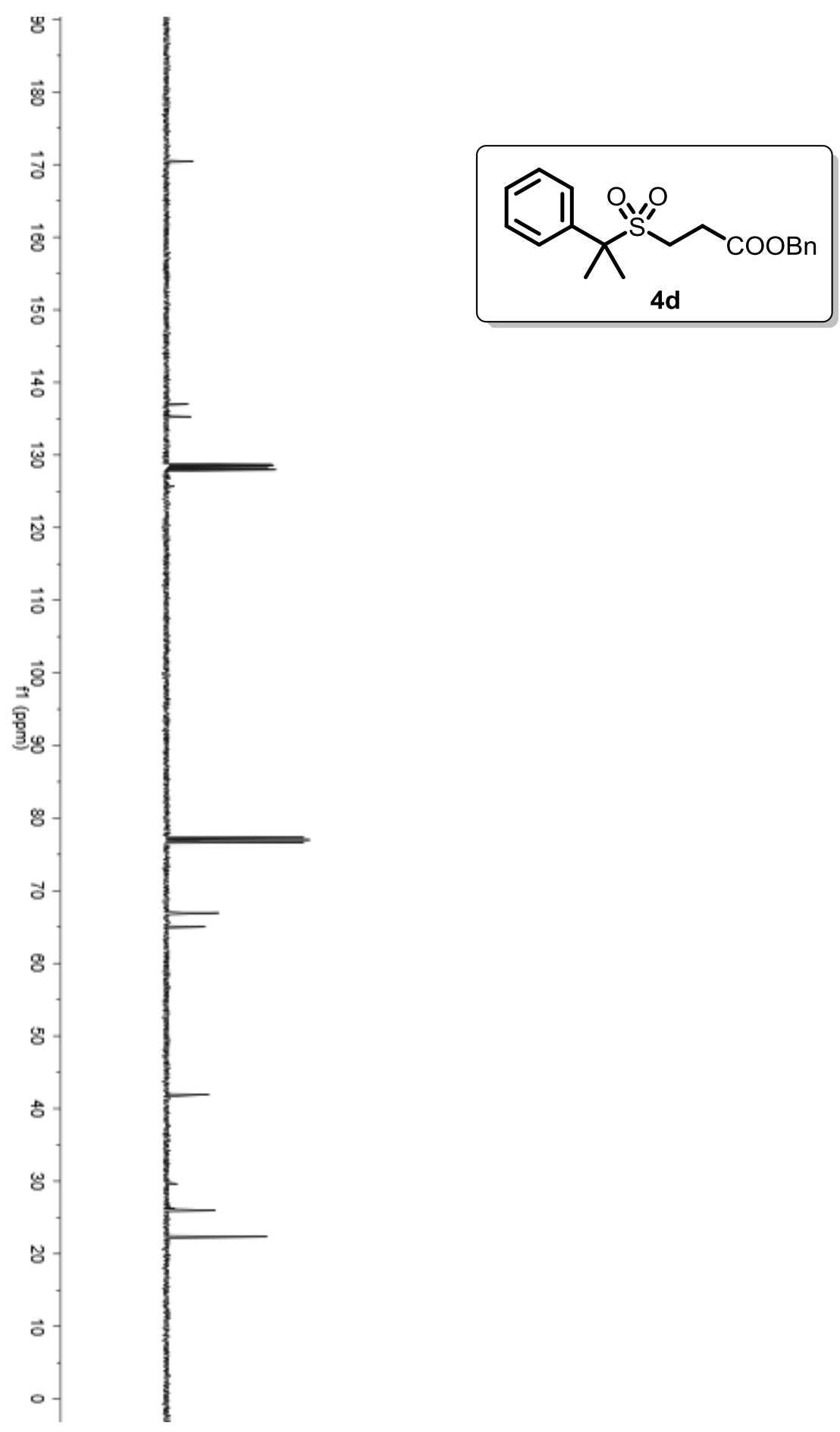

$-170.43$

136.99

135.29

- 128.75

128.66

128.53

128.35

-128.17

$\lcm{127.97}$

$-66.95$ 
${ }^{1} \mathbf{H}$ NMR $\left(400 \mathrm{MHz}, \mathrm{CDCl}_{3}\right)$

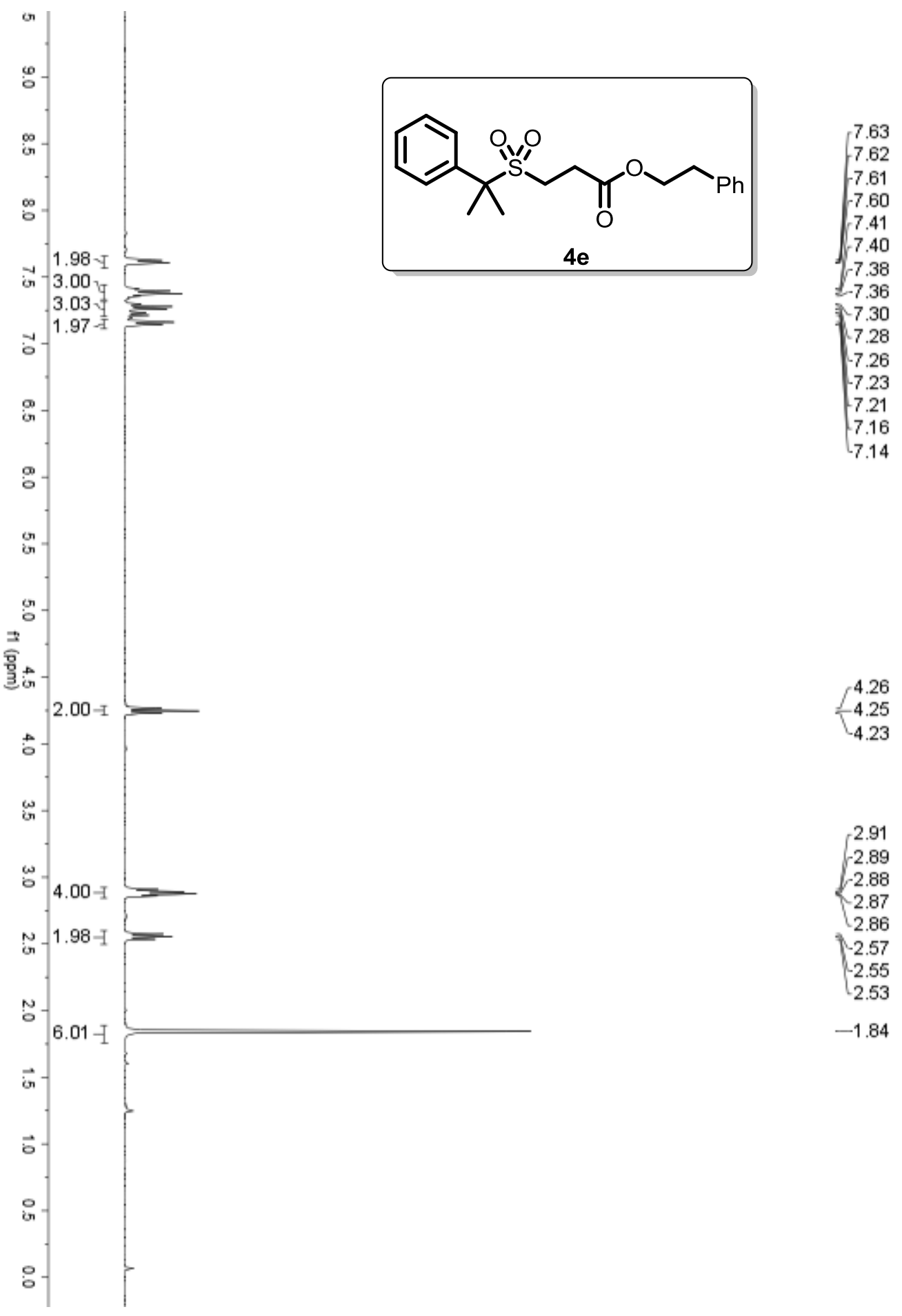


${ }^{13} \mathrm{C}$ NMR $\left(100 \mathrm{MHz}, \mathrm{CDCl}_{3}\right)$

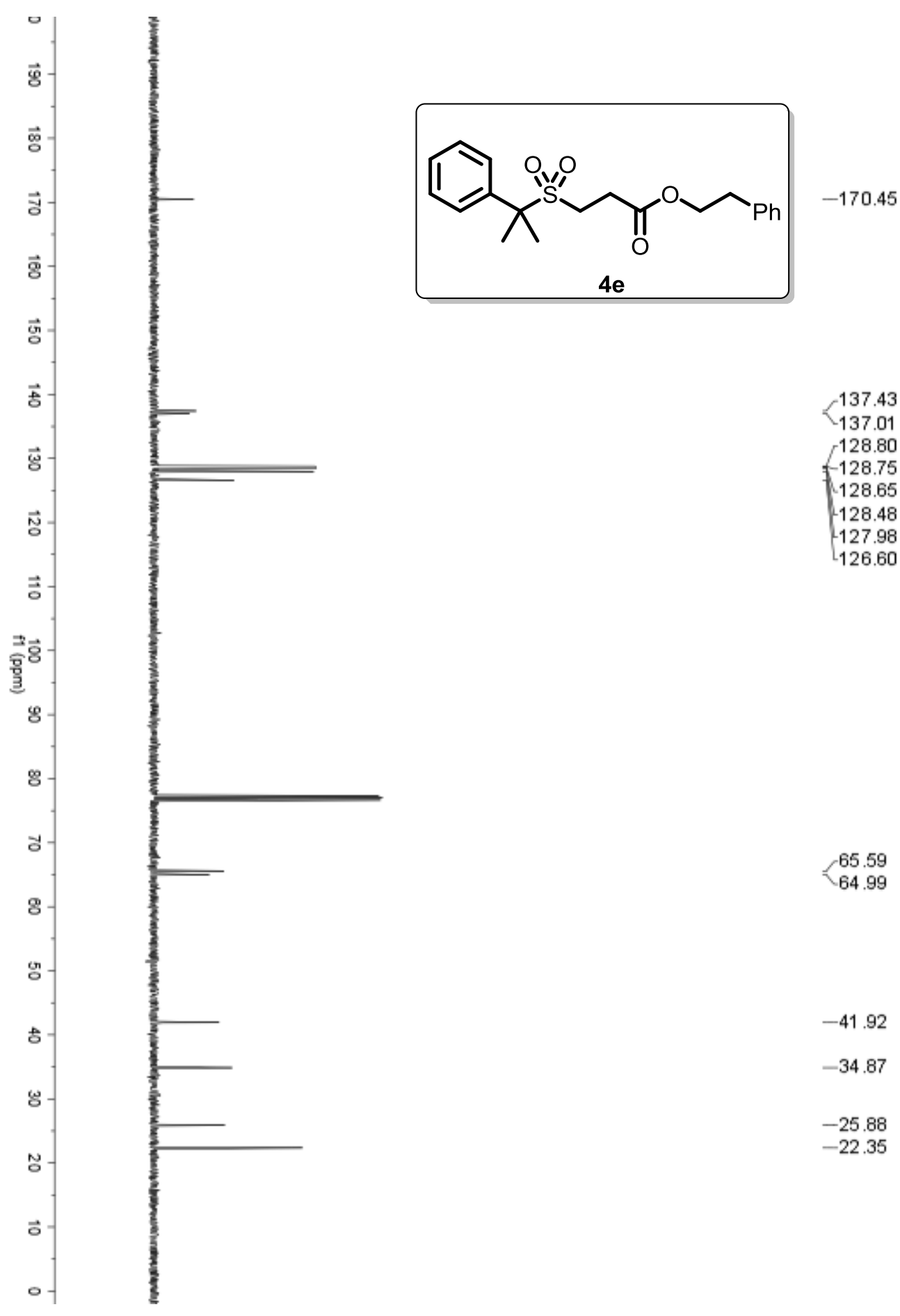


${ }^{1} \mathbf{H}$ NMR $\left(400 \mathrm{MHz}, \mathrm{CDCl}_{3}\right)$

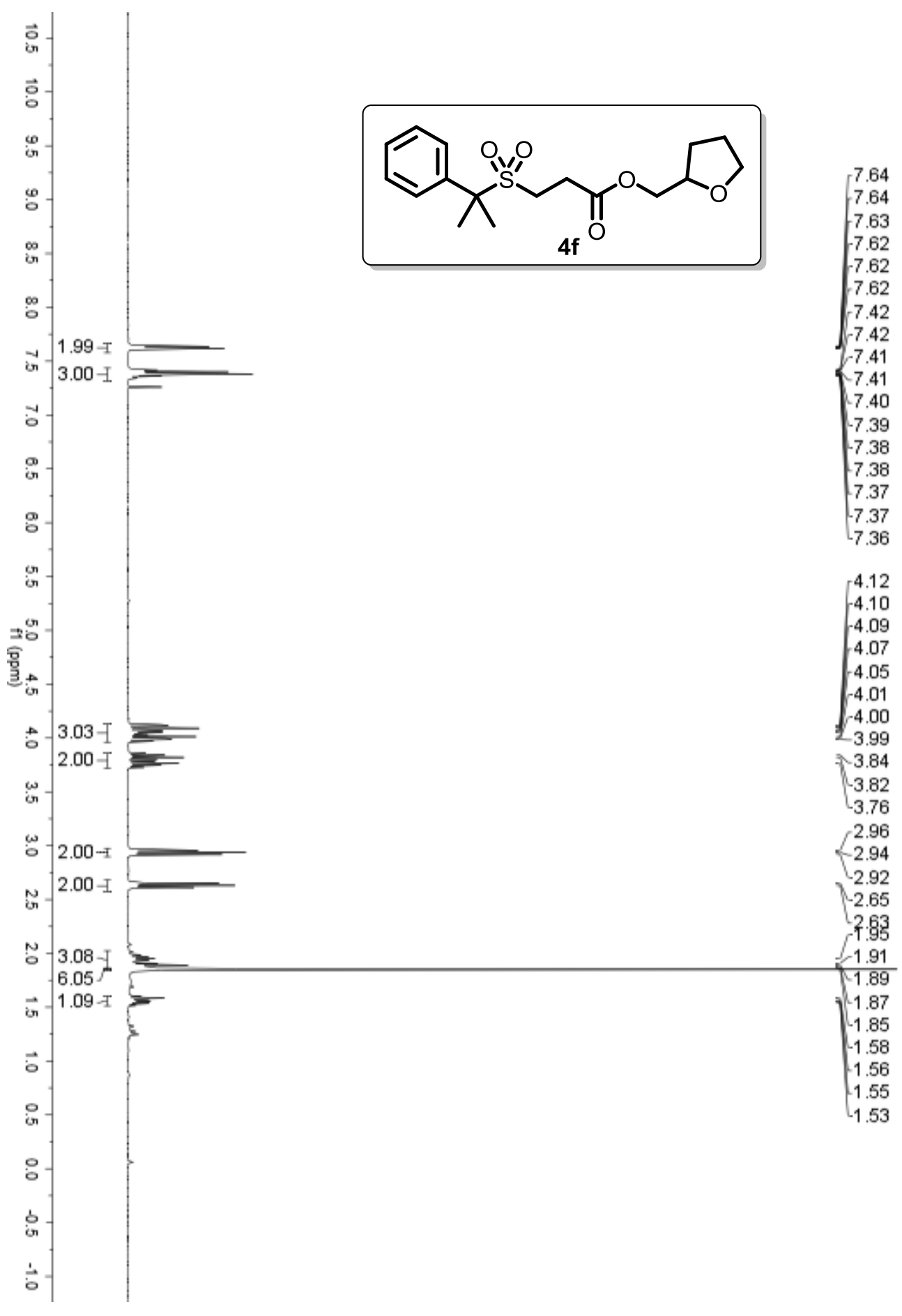


${ }^{13} \mathrm{C}$ NMR $\left(100 \mathrm{MHz}, \mathrm{CDCl}_{3}\right)$

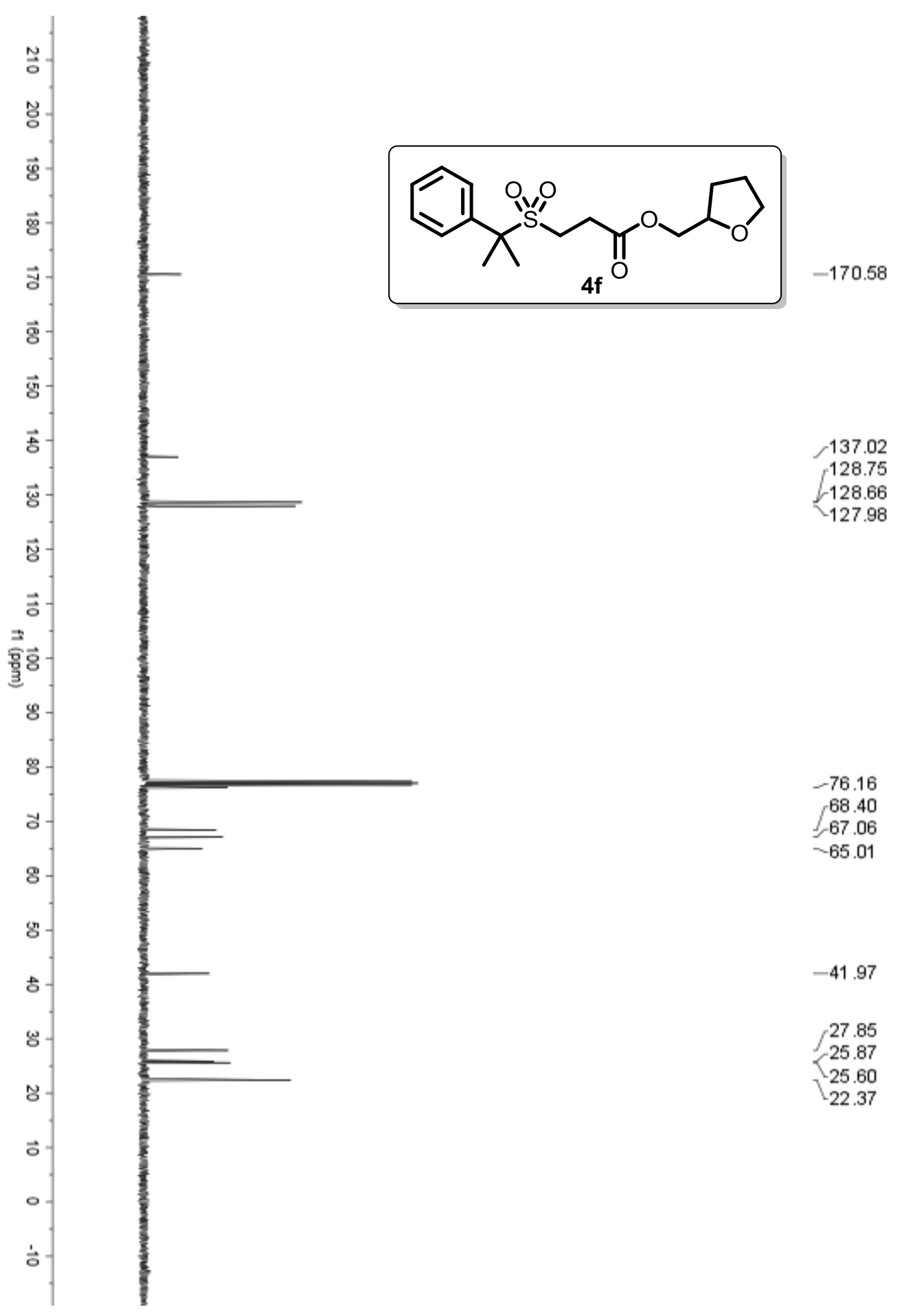


${ }^{1} \mathbf{H}$ NMR $\left(400 \mathrm{MHz}, \mathrm{CDCl}_{3}\right)$

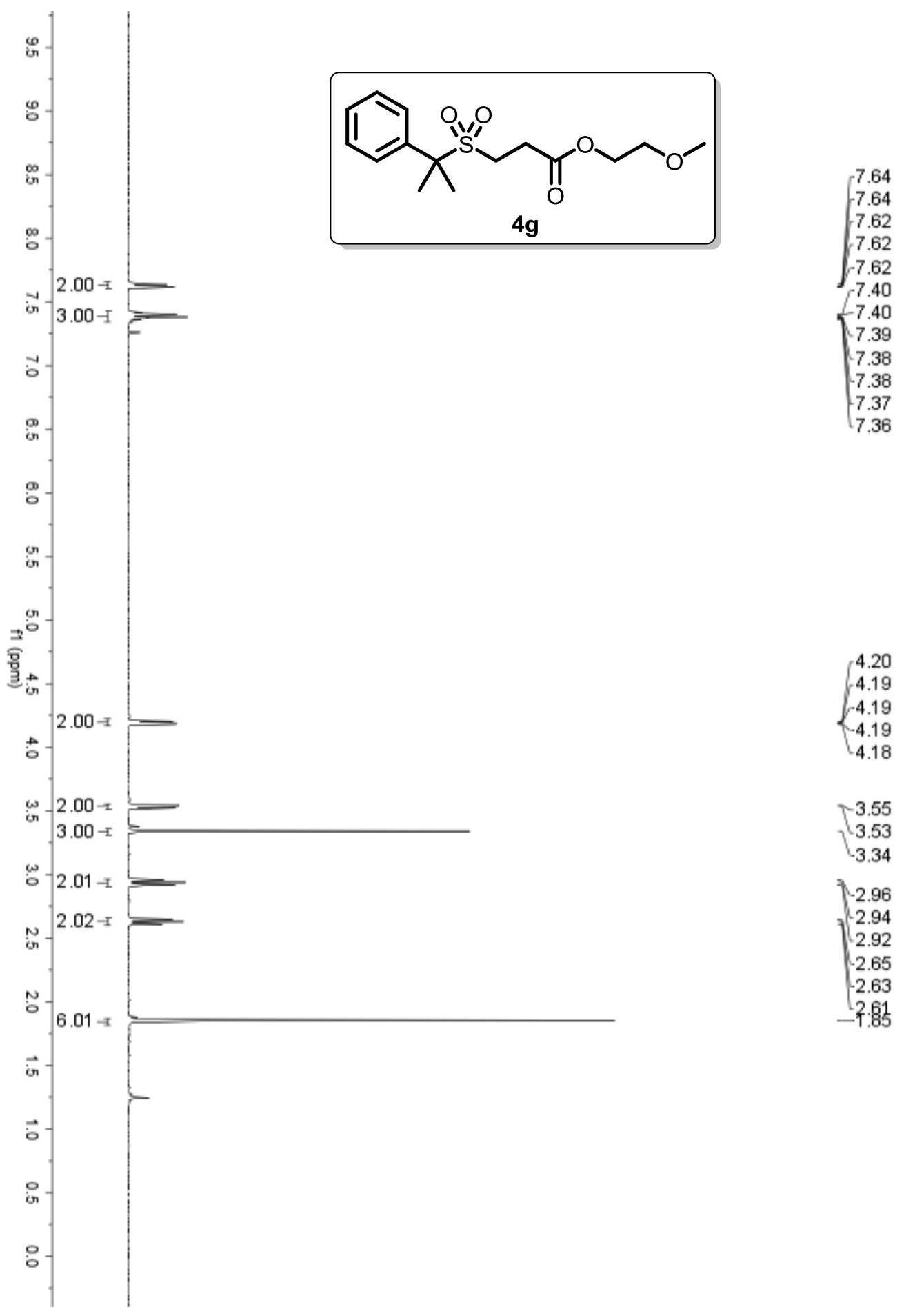


${ }^{13} \mathrm{C}$ NMR $\left(100 \mathrm{MHz}, \mathrm{CDCl}_{3}\right)$

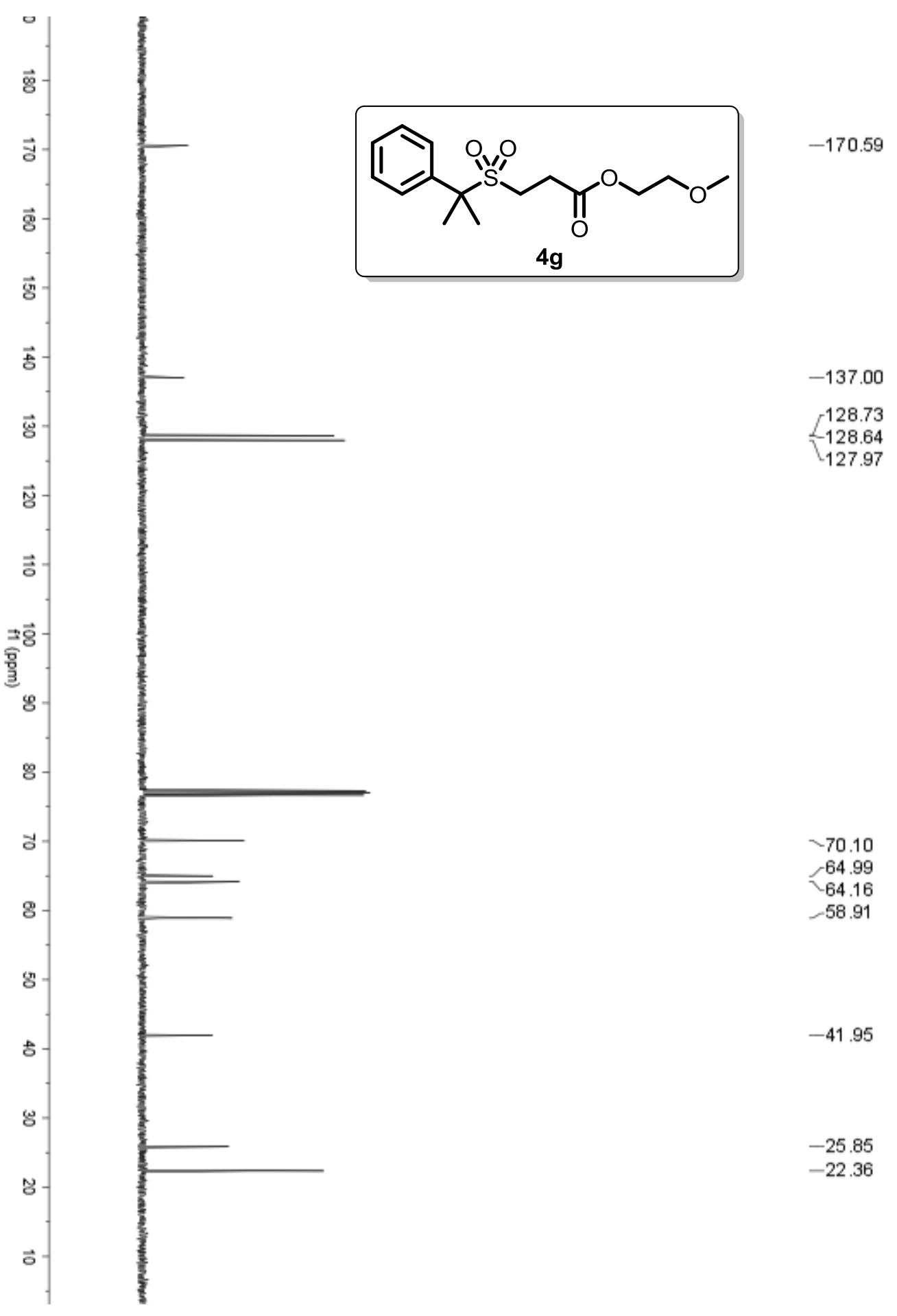


${ }^{1} \mathbf{H}$ NMR $\left(400 \mathrm{MHz}, \mathrm{CDCl}_{3}\right)$

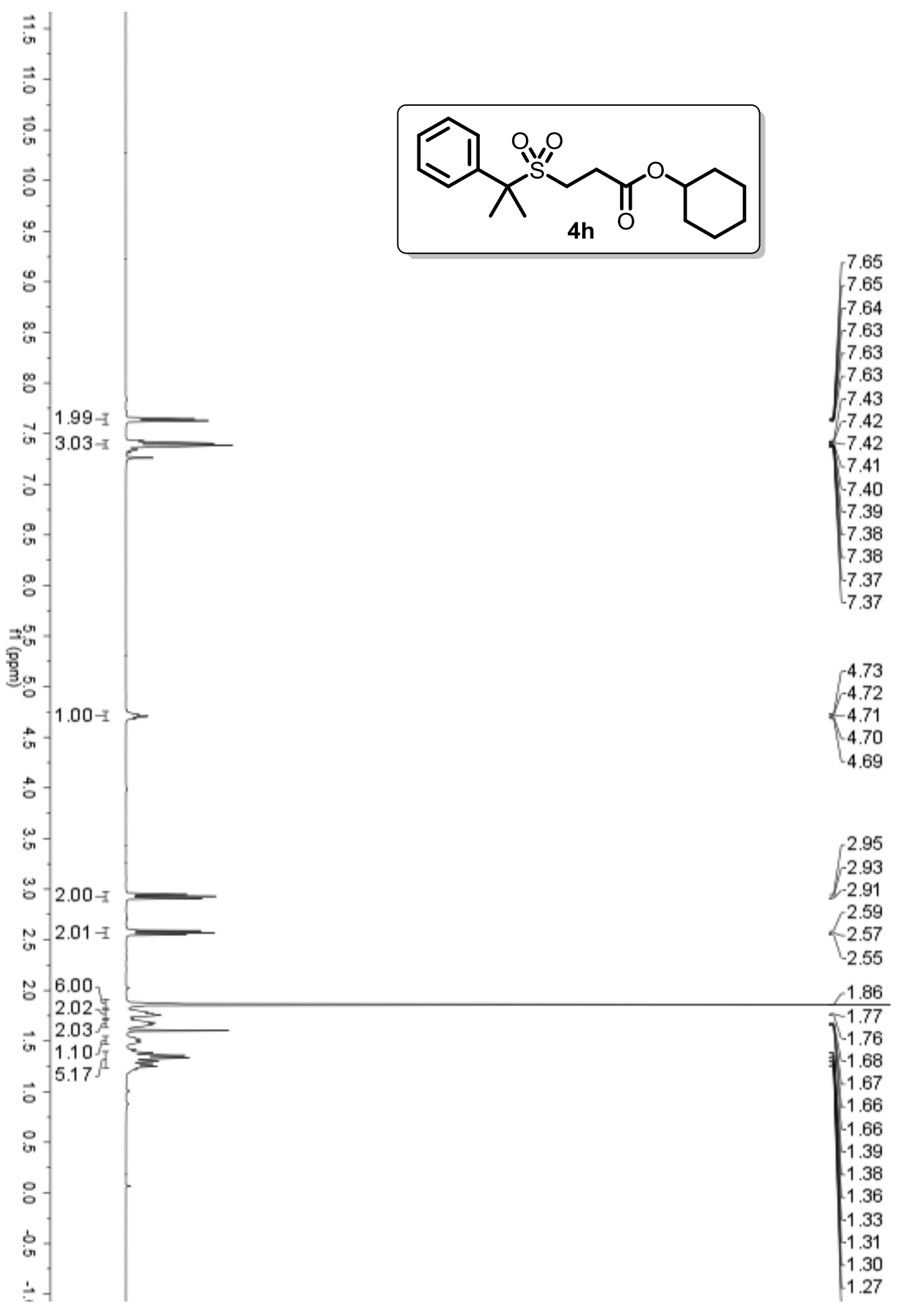


${ }^{13} \mathrm{C}$ NMR $\left(100 \mathrm{MHz}, \mathrm{CDCl}_{3}\right)$

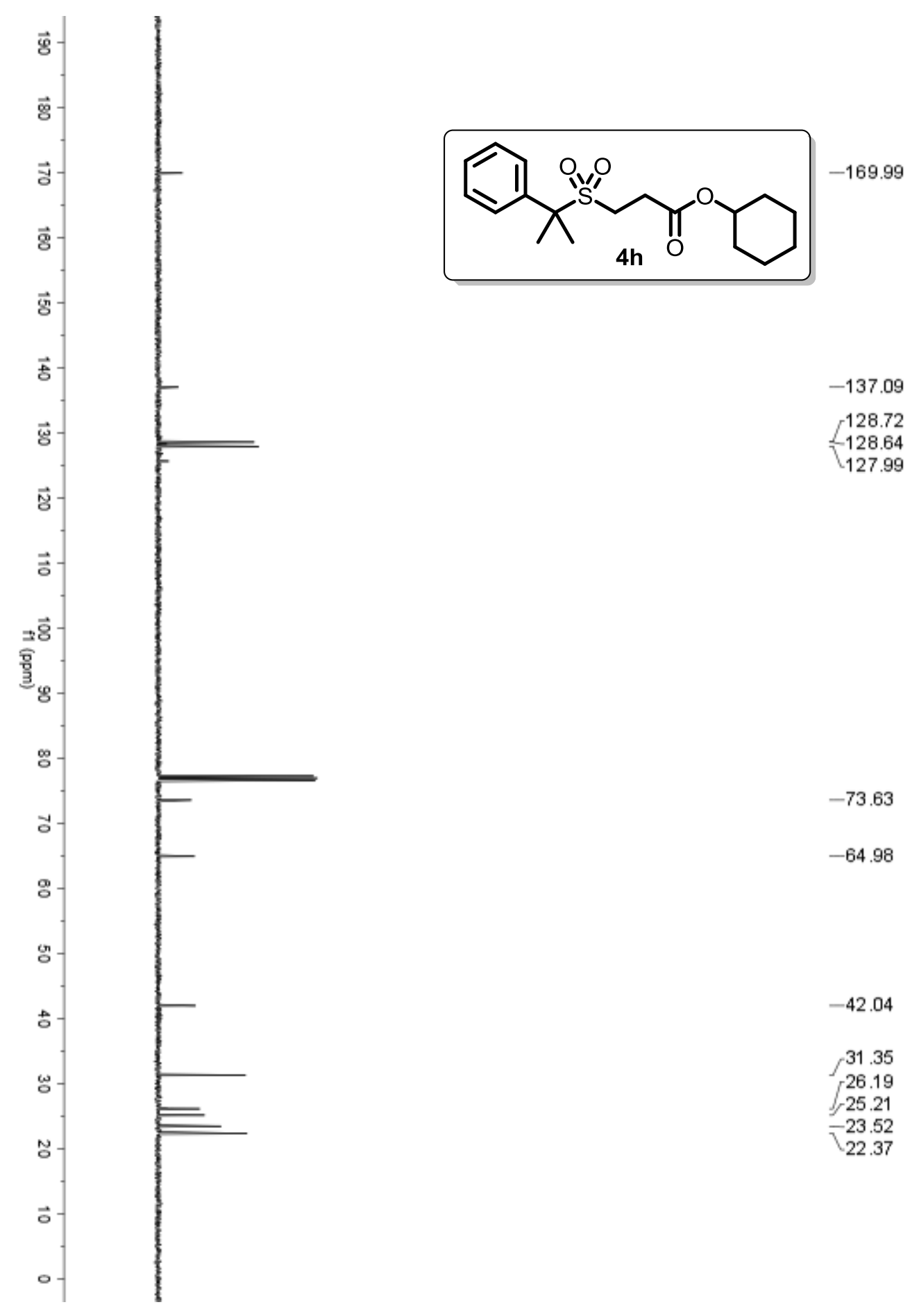


${ }^{1} \mathbf{H}$ NMR $\left(400 \mathrm{MHz}, \mathrm{CDCl}_{3}\right)$

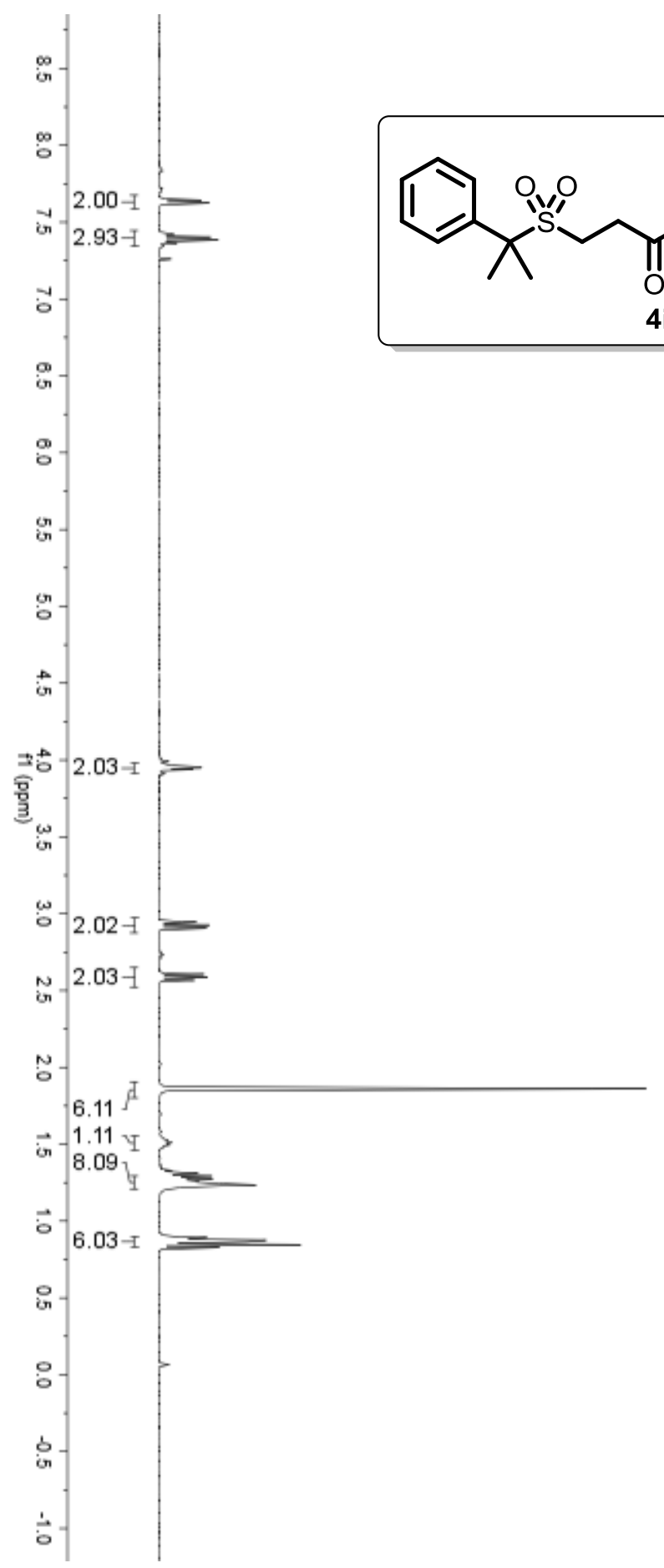


${ }^{13} \mathrm{C}$ NMR $\left(100 \mathrm{MHz}, \mathrm{CDCl}_{3}\right)$

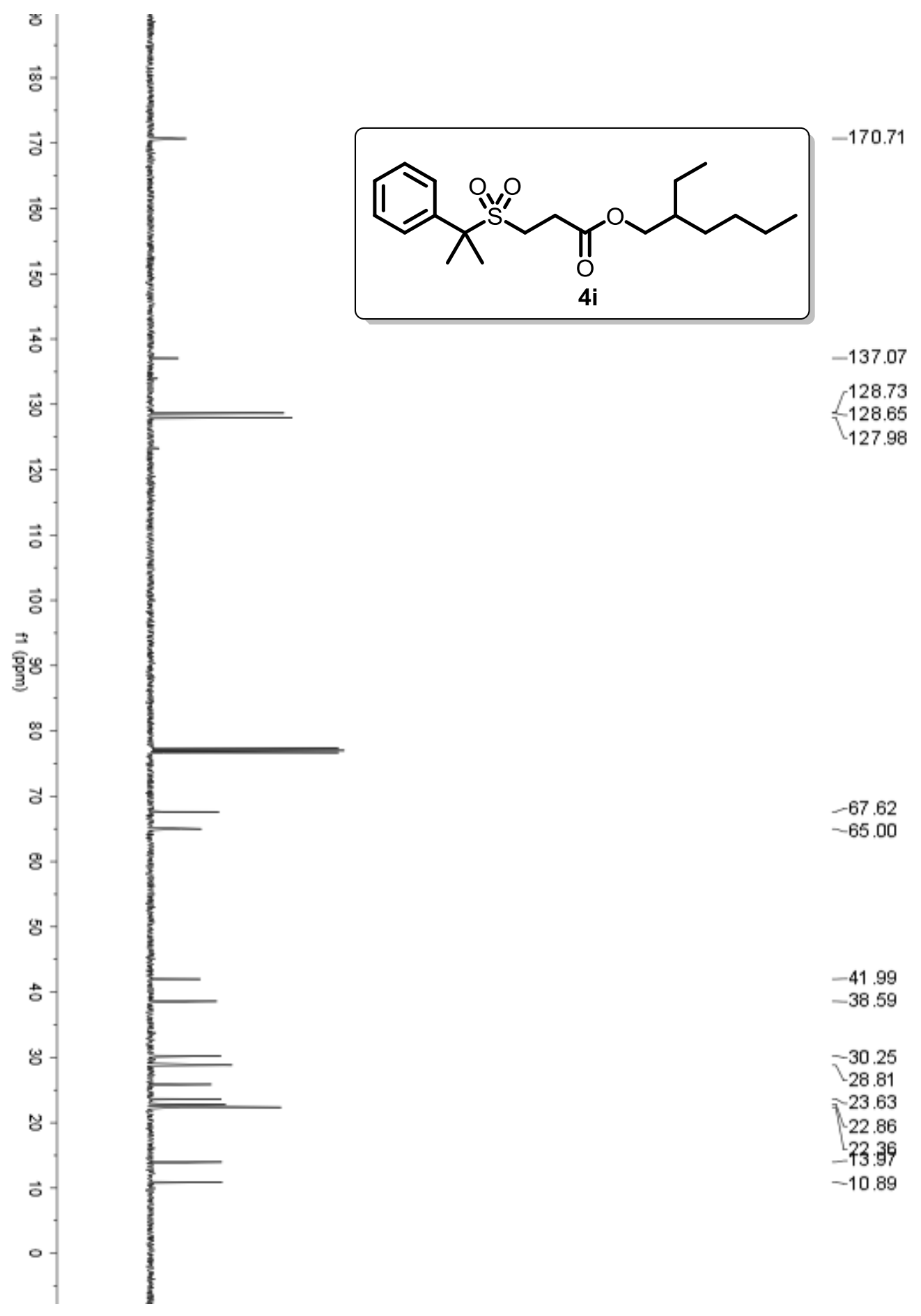


${ }^{1} \mathbf{H}$ NMR $\left(400 \mathrm{MHz}, \mathrm{CDCl}_{3}\right)$

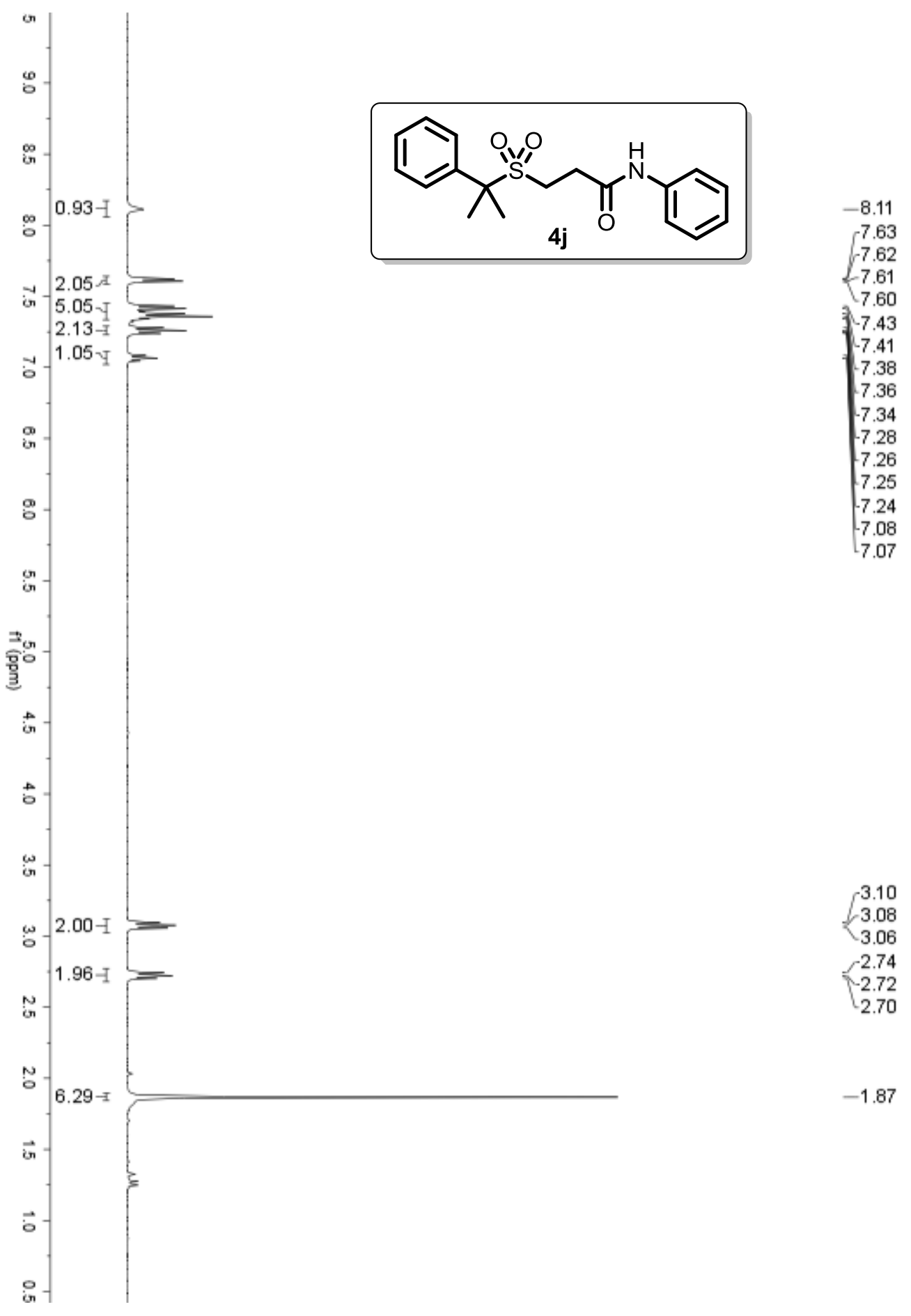


${ }^{13} \mathrm{C}$ NMR $\left(100 \mathrm{MHz}, \mathrm{CDCl}_{3}\right)$

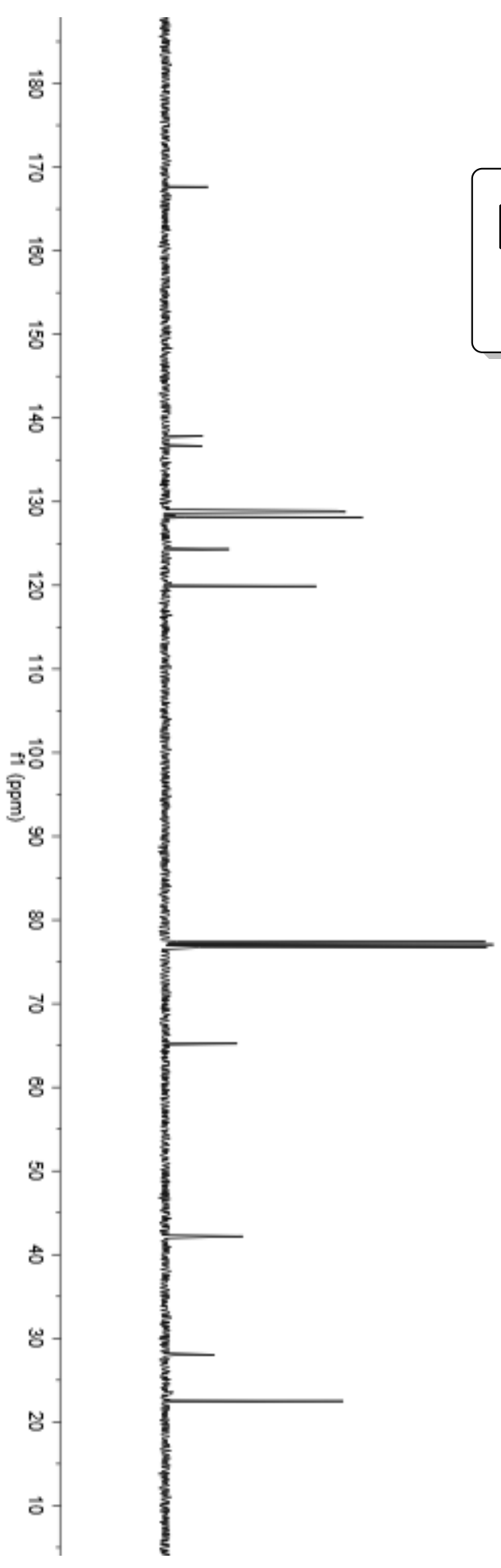

$-167.65$

$-137.84$

$-136.67$

128.91

$-128.77$

$-128.13$

124.33

$\backslash 119.91$

$-65.21$

$-42.19$

$-28.03$

$-22.49$ 
${ }^{1} \mathbf{H}$ NMR $\left(400 \mathrm{MHz}, \mathrm{CDCl}_{3}\right)$

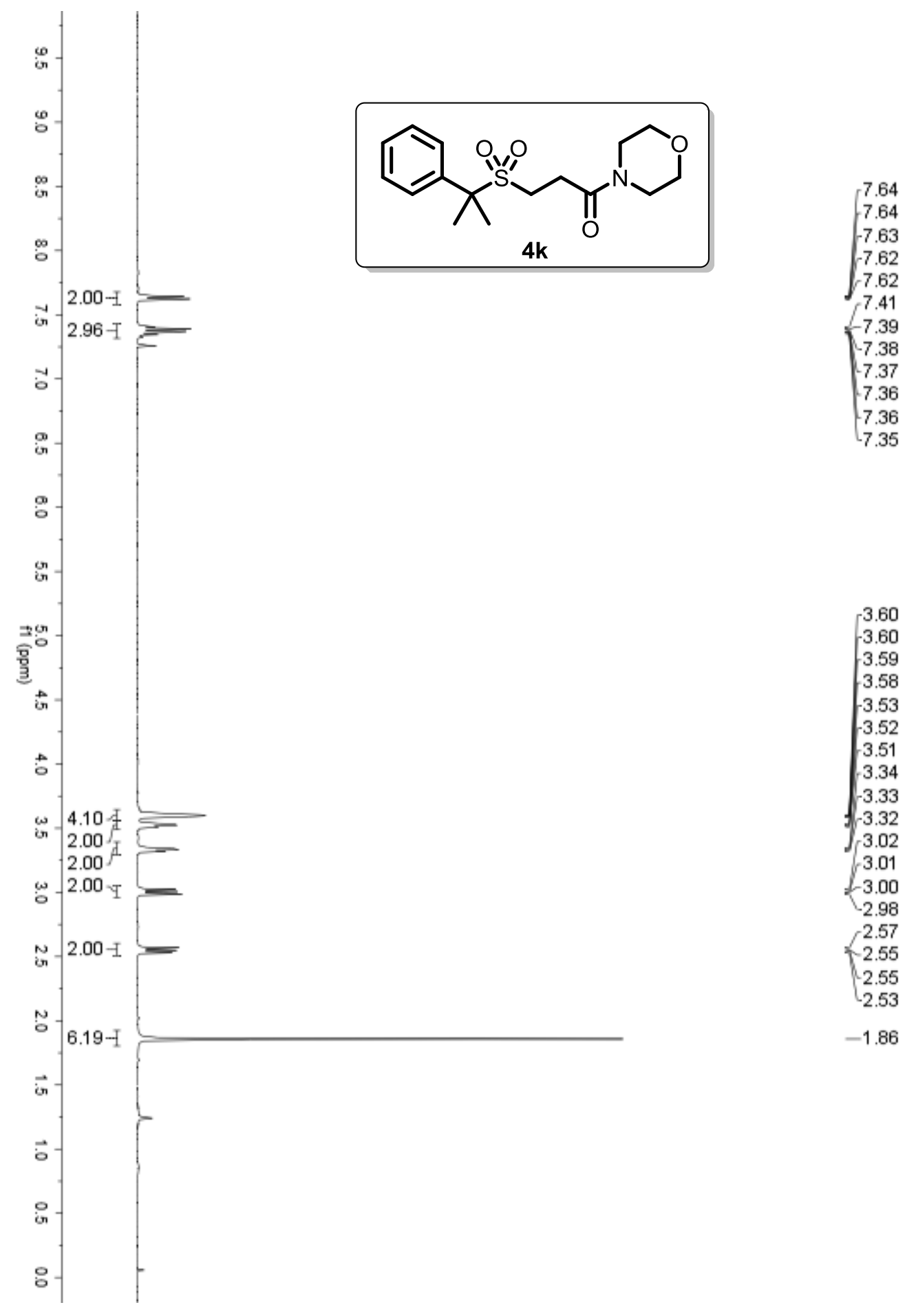


${ }^{13} \mathrm{C}$ NMR $\left(100 \mathrm{MHz}, \mathrm{CDCl}_{3}\right)$

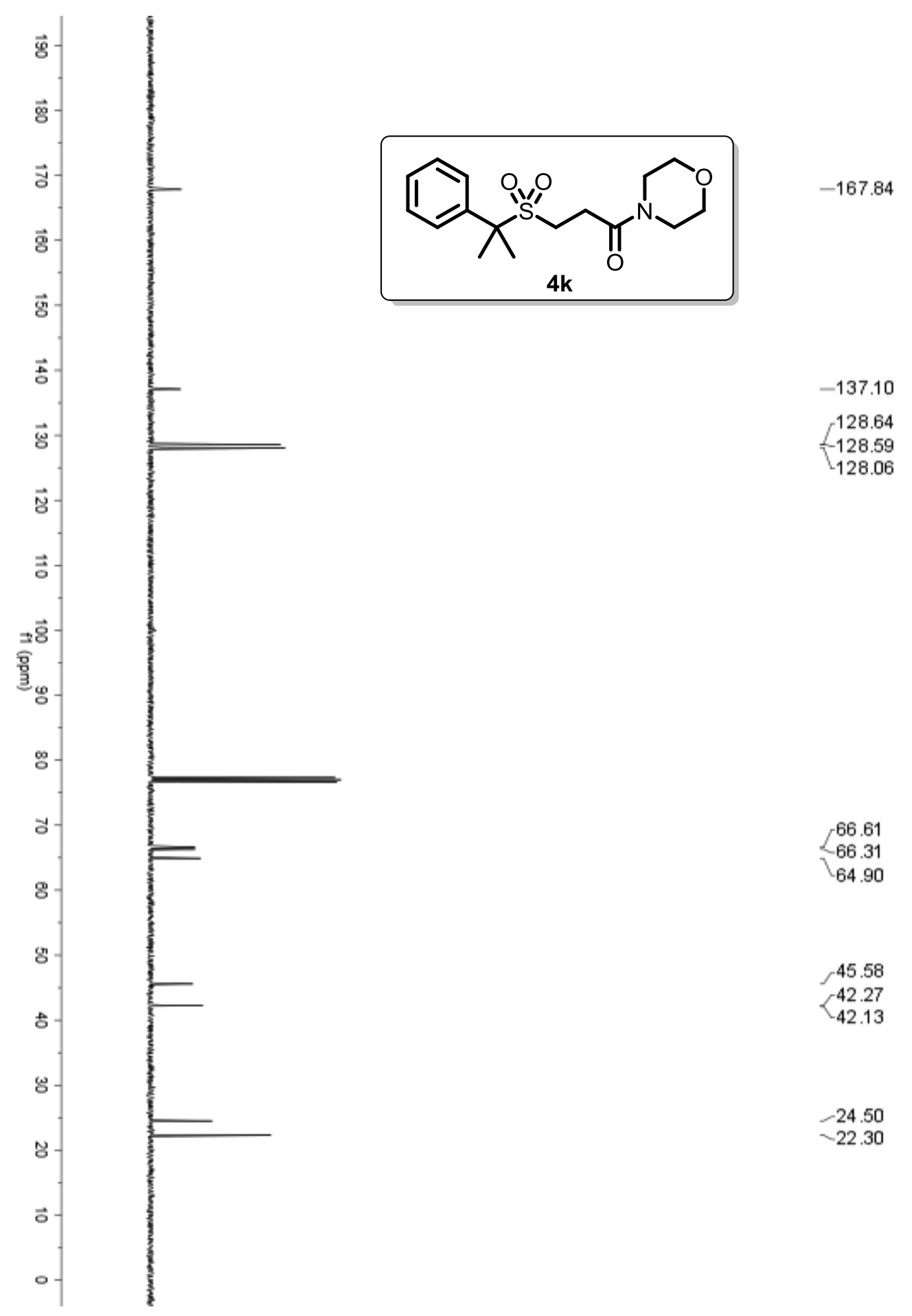


${ }^{1} \mathrm{H}$ NMR $\left(400 \mathrm{MHz}, \mathrm{CDCl}_{3}\right)$

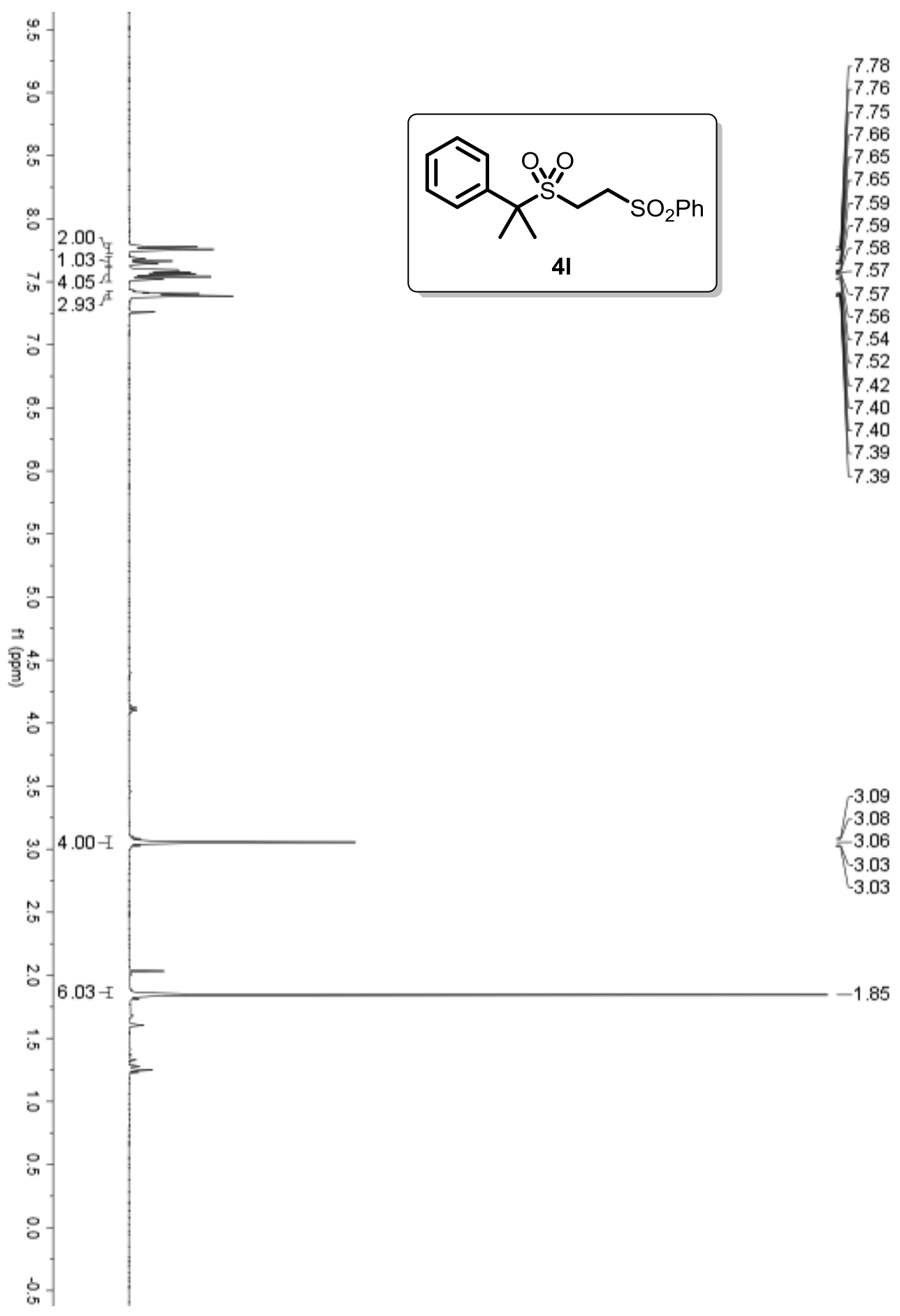


${ }^{13} \mathrm{C}$ NMR $\left(100 \mathrm{MHz}, \mathrm{CDCl}_{3}\right)$

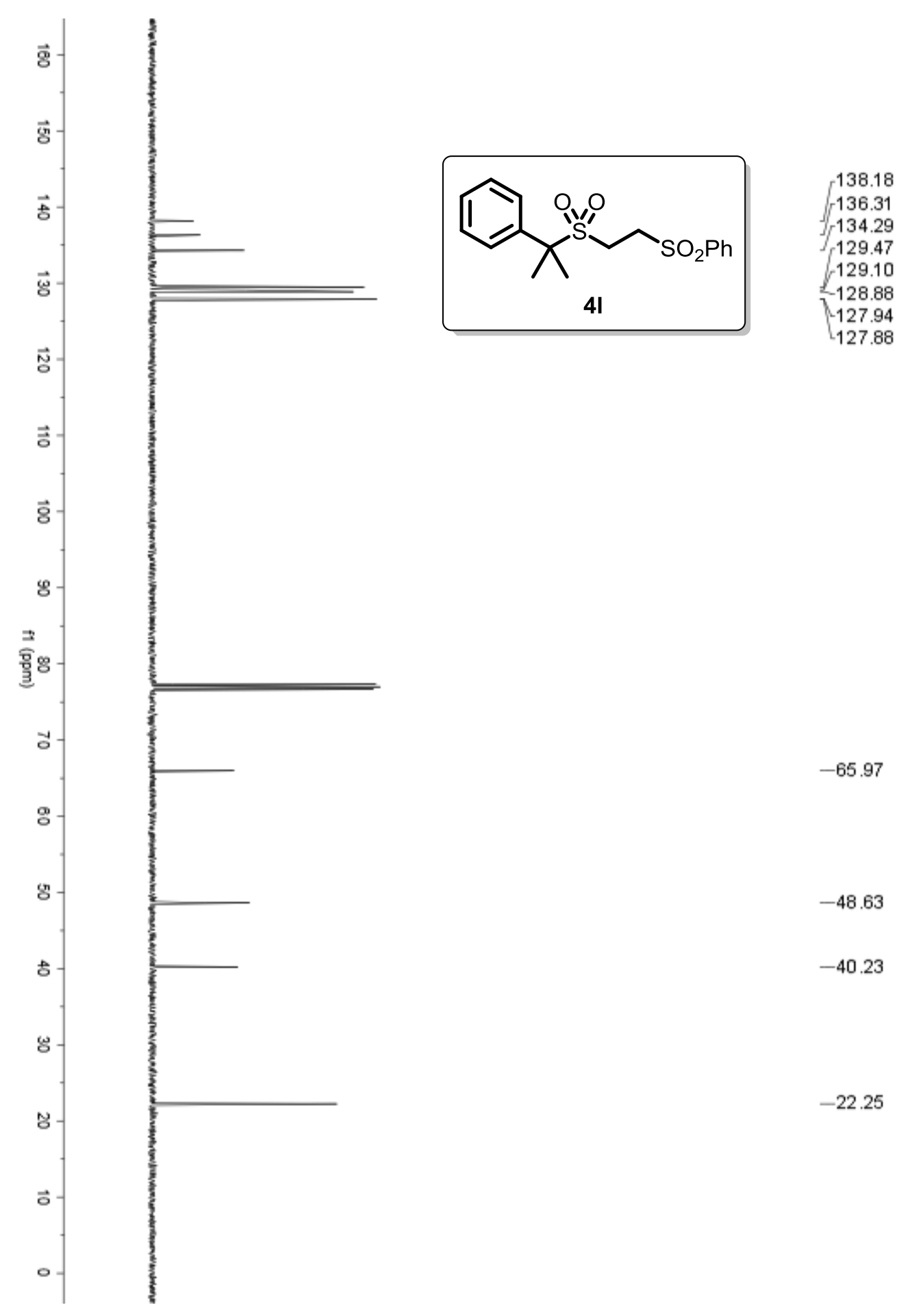


${ }^{1} \mathbf{H}$ NMR $\left(400 \mathrm{MHz}, \mathrm{CDCl}_{3}\right)$

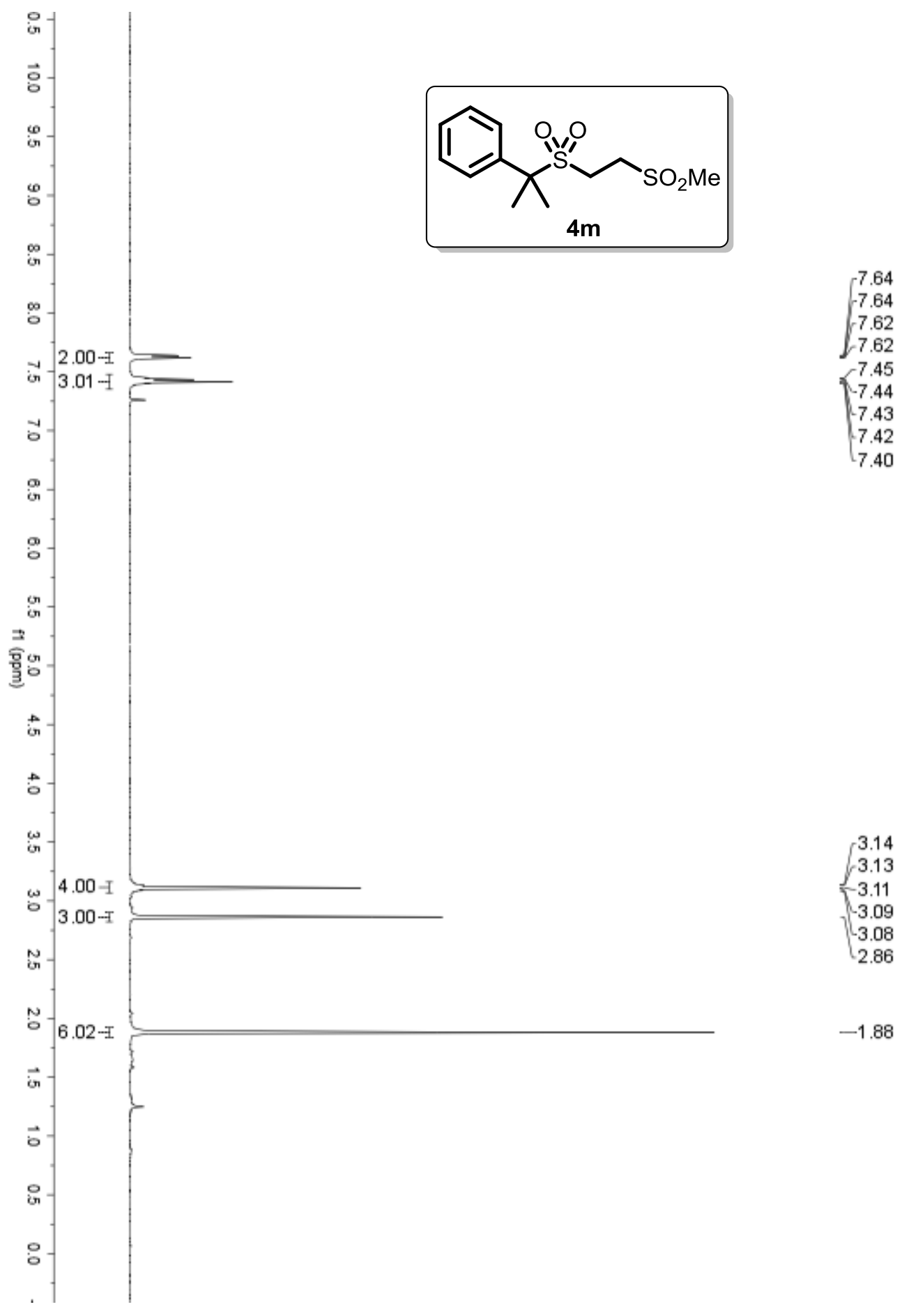


${ }^{13} \mathrm{C}$ NMR $\left(100 \mathrm{MHz}, \mathrm{CDCl}_{3}\right)$

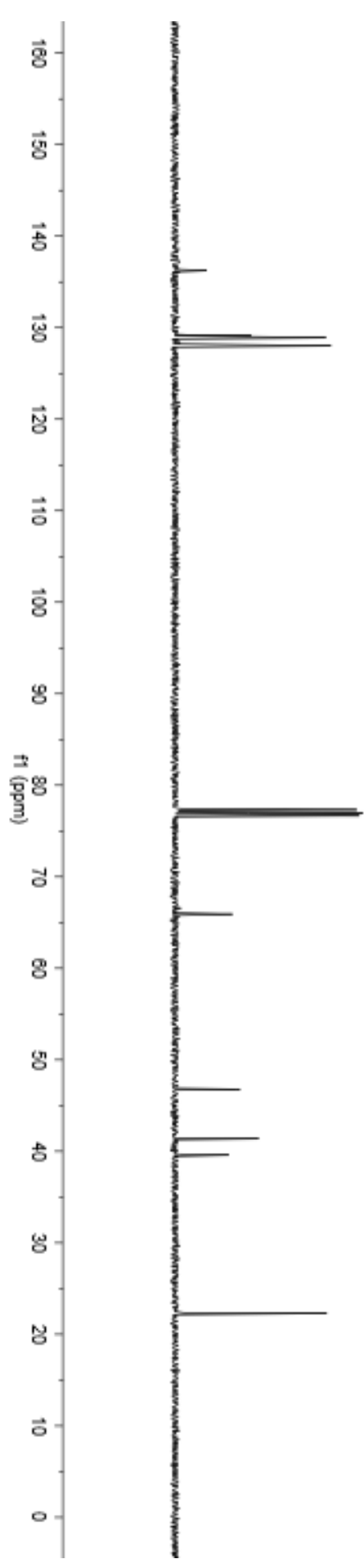

$-136.25$

129.17

$<128.91$

$\backslash 128.02$

$-65.92$

$\checkmark 46.77$

41.38

$-39.59$

$-22.31$ 
${ }^{1} \mathbf{H}$ NMR $\left(400 \mathrm{MHz}, \mathrm{CDCl}_{3}\right)$

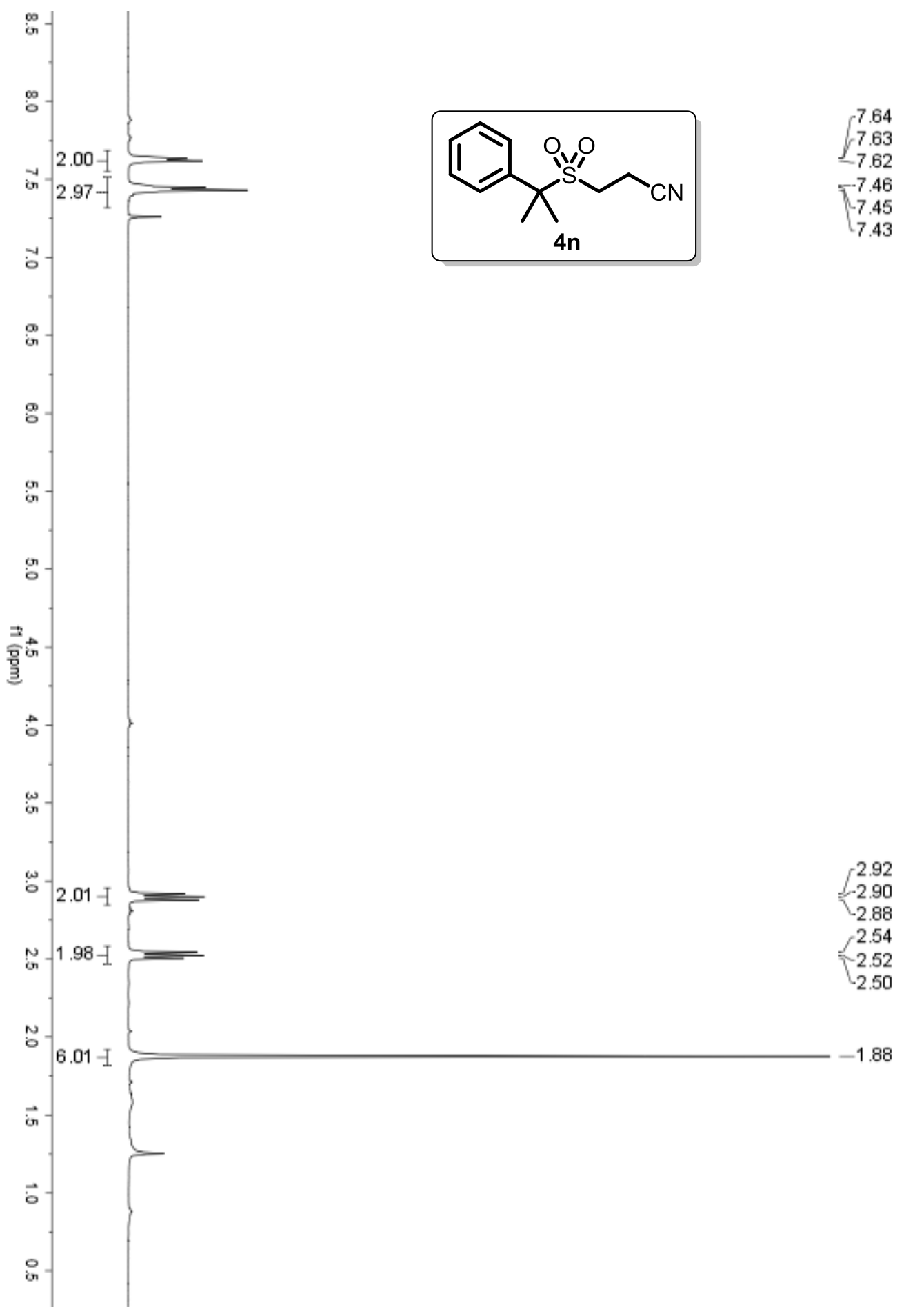


${ }^{13} \mathrm{C}$ NMR $\left(100 \mathrm{MHz}, \mathrm{CDCl}_{3}\right)$

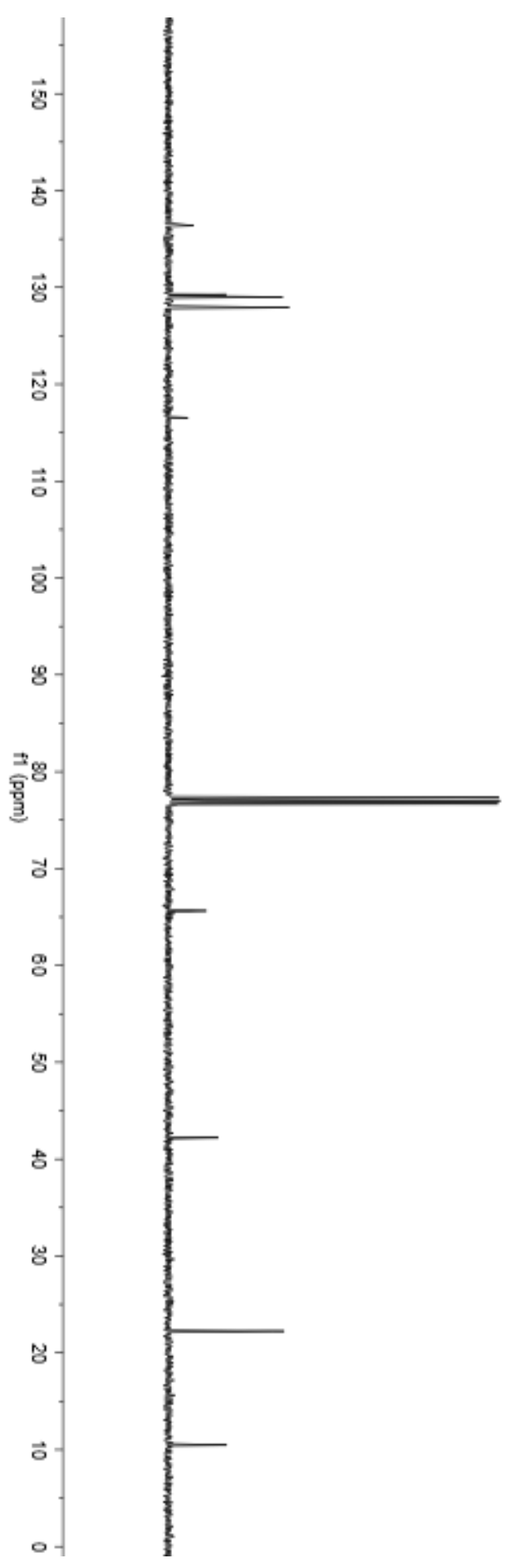

$-136.37$

$r^{129.26}$

$<129.00$

$-127.91$

$-116.55$

$-65.64$

$-42.21$

$-22.24$

$-10.47$ 
${ }^{1} \mathbf{H}$ NMR $\left(400 \mathrm{MHz}, \mathrm{CDCl}_{3}\right)$

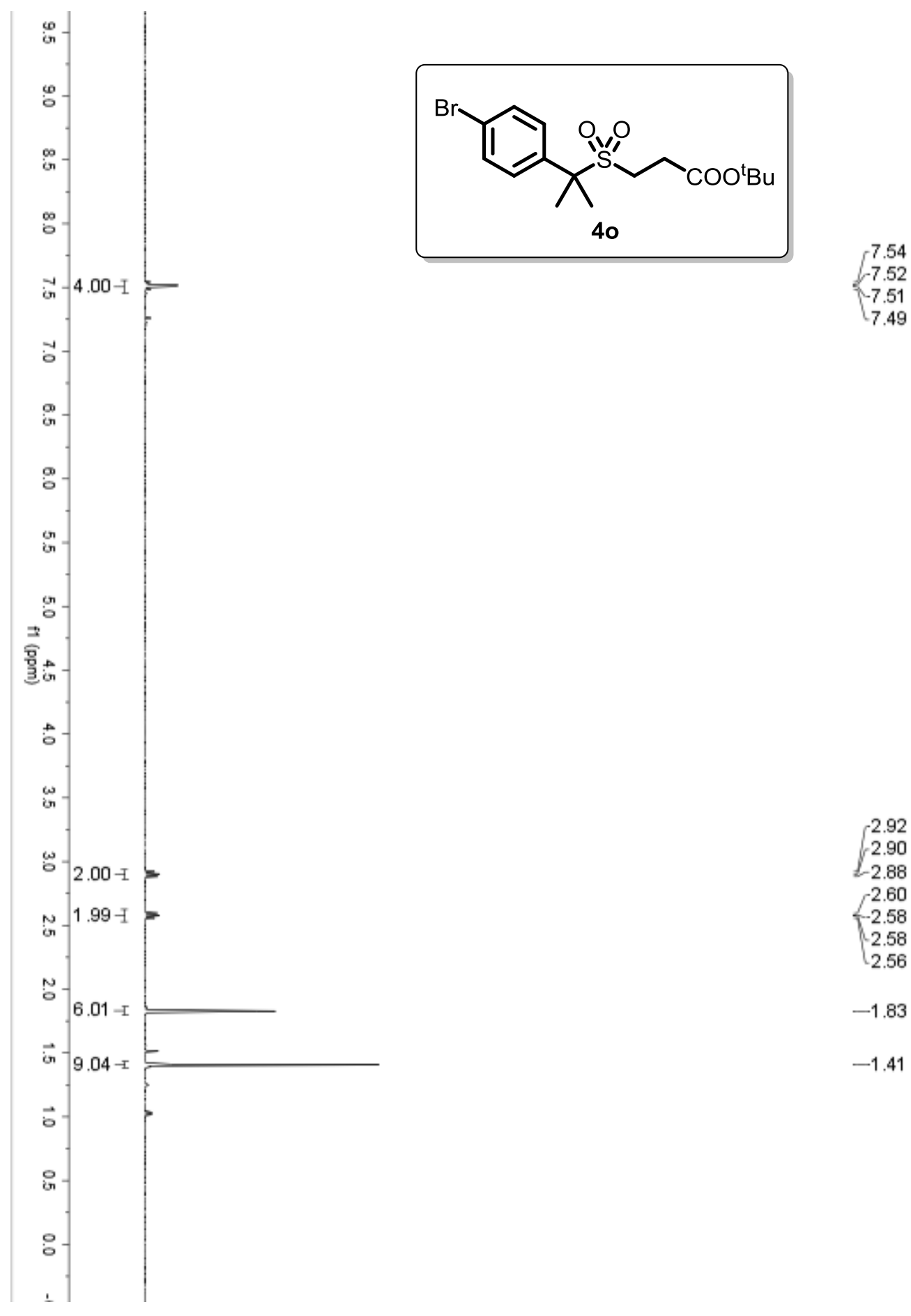


${ }^{13} \mathrm{C}$ NMR $\left(100 \mathrm{MHz}, \mathrm{CDCl}_{3}\right)$

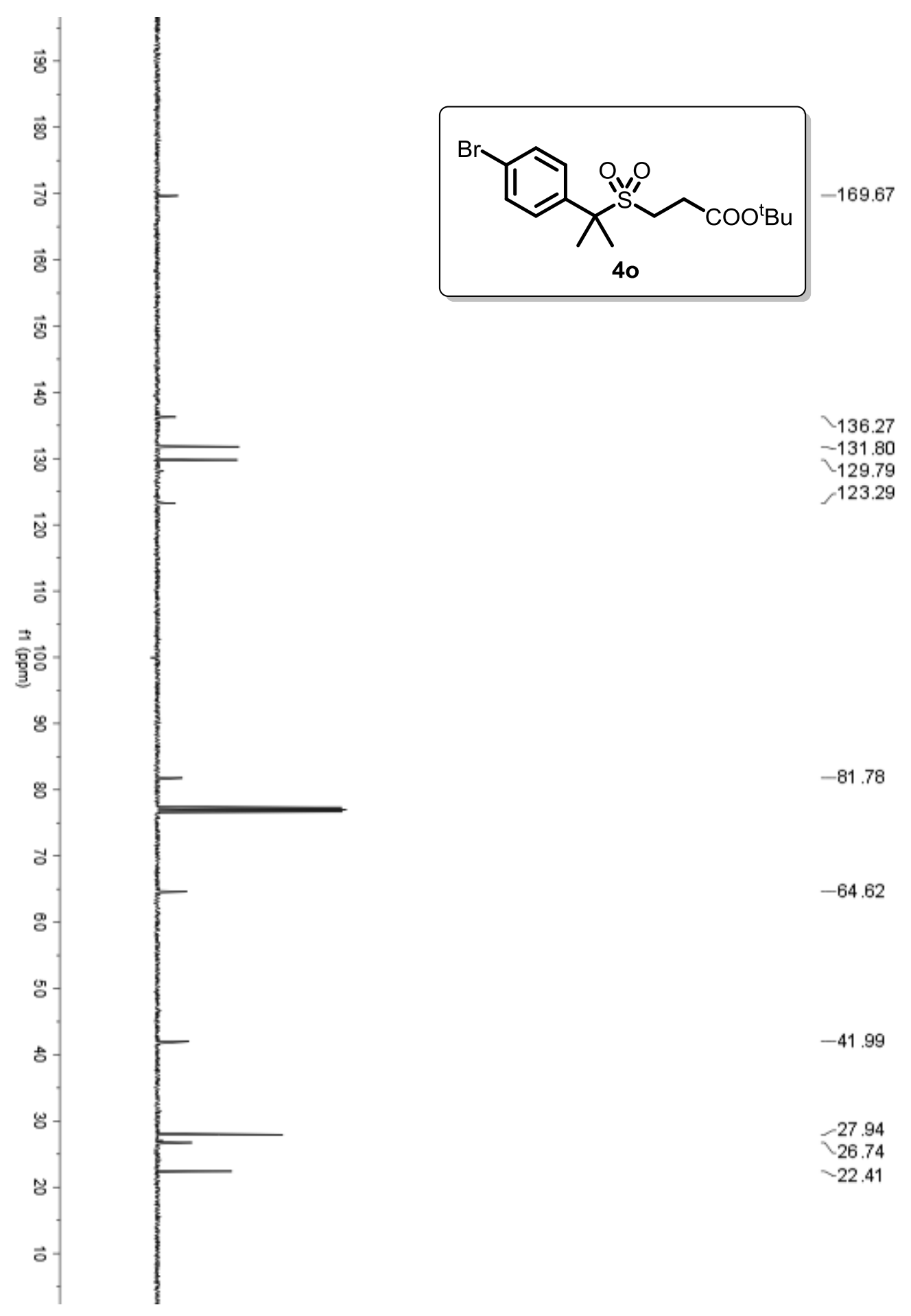


${ }^{1} \mathbf{H}$ NMR $\left(400 \mathrm{MHz}, \mathrm{CDCl}_{3}\right)$

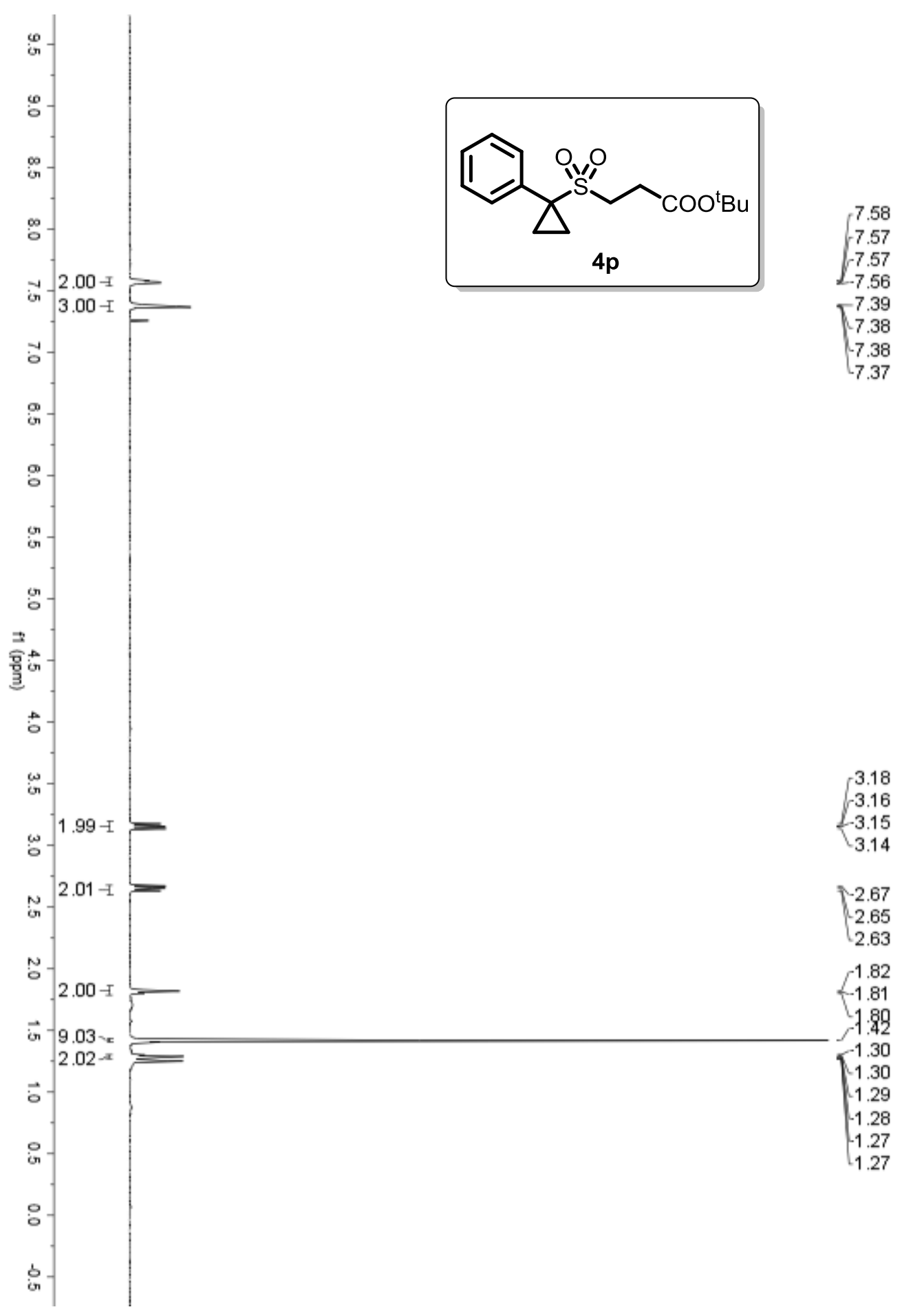


${ }^{13} \mathrm{C}$ NMR $\left(100 \mathrm{MHz}, \mathrm{CDCl}_{3}\right)$
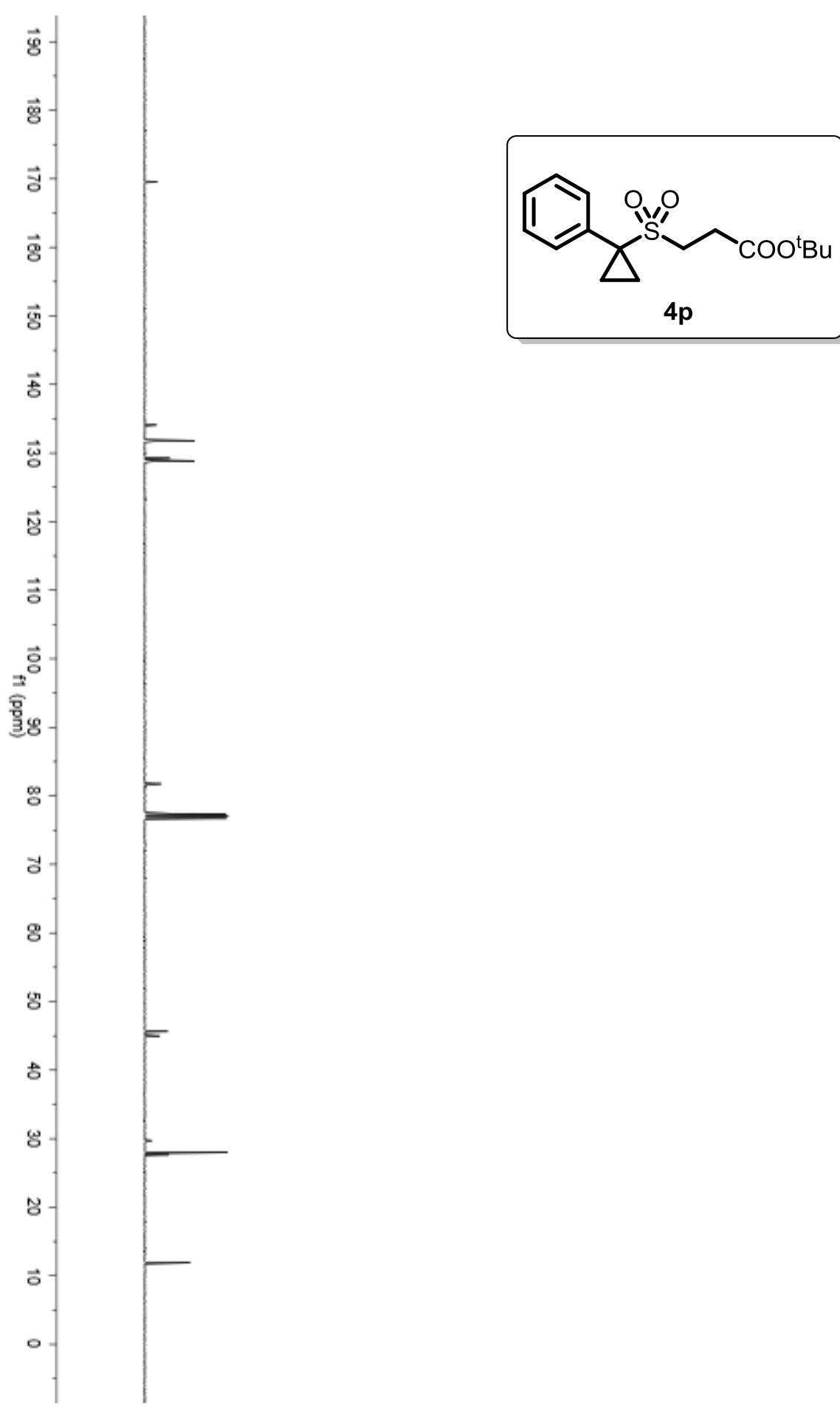

$-169.55$

134.09

131.81

$-129.24$

$\backslash 128.86$

$-81.76$

$-45.63$

$-44.99$

$<\begin{array}{r}27.99 \\ 27.65\end{array}$

$-11.91$ 
${ }^{1} \mathbf{H}$ NMR $\left(400 \mathrm{MHz}, \mathrm{CDCl}_{3}\right)$

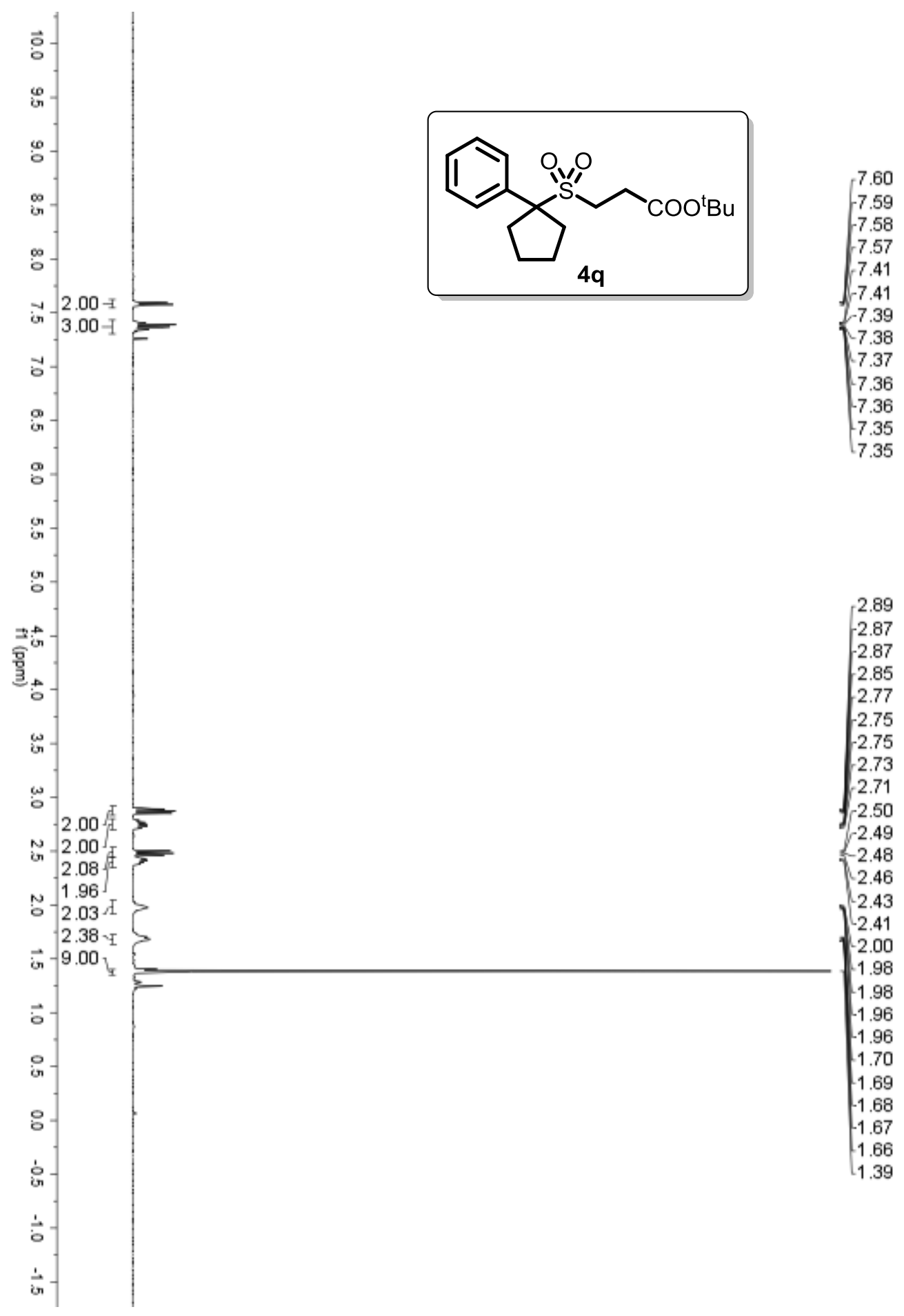


${ }^{13} \mathrm{C}$ NMR $\left(100 \mathrm{MHz}, \mathrm{CDCl}_{3}\right)$

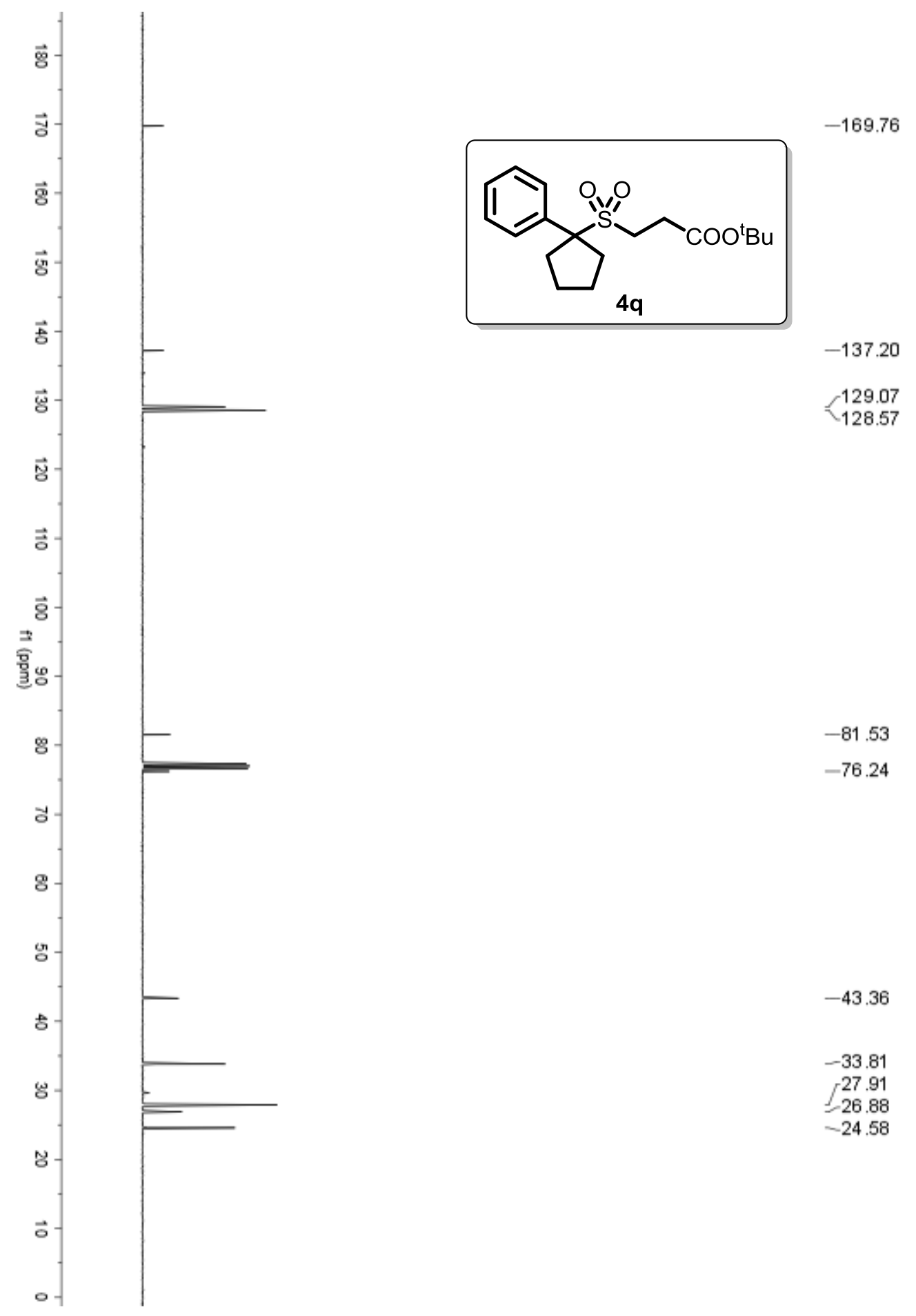


${ }^{1} \mathbf{H}$ NMR $\left(400 \mathrm{MHz}, \mathrm{CDCl}_{3}\right)$

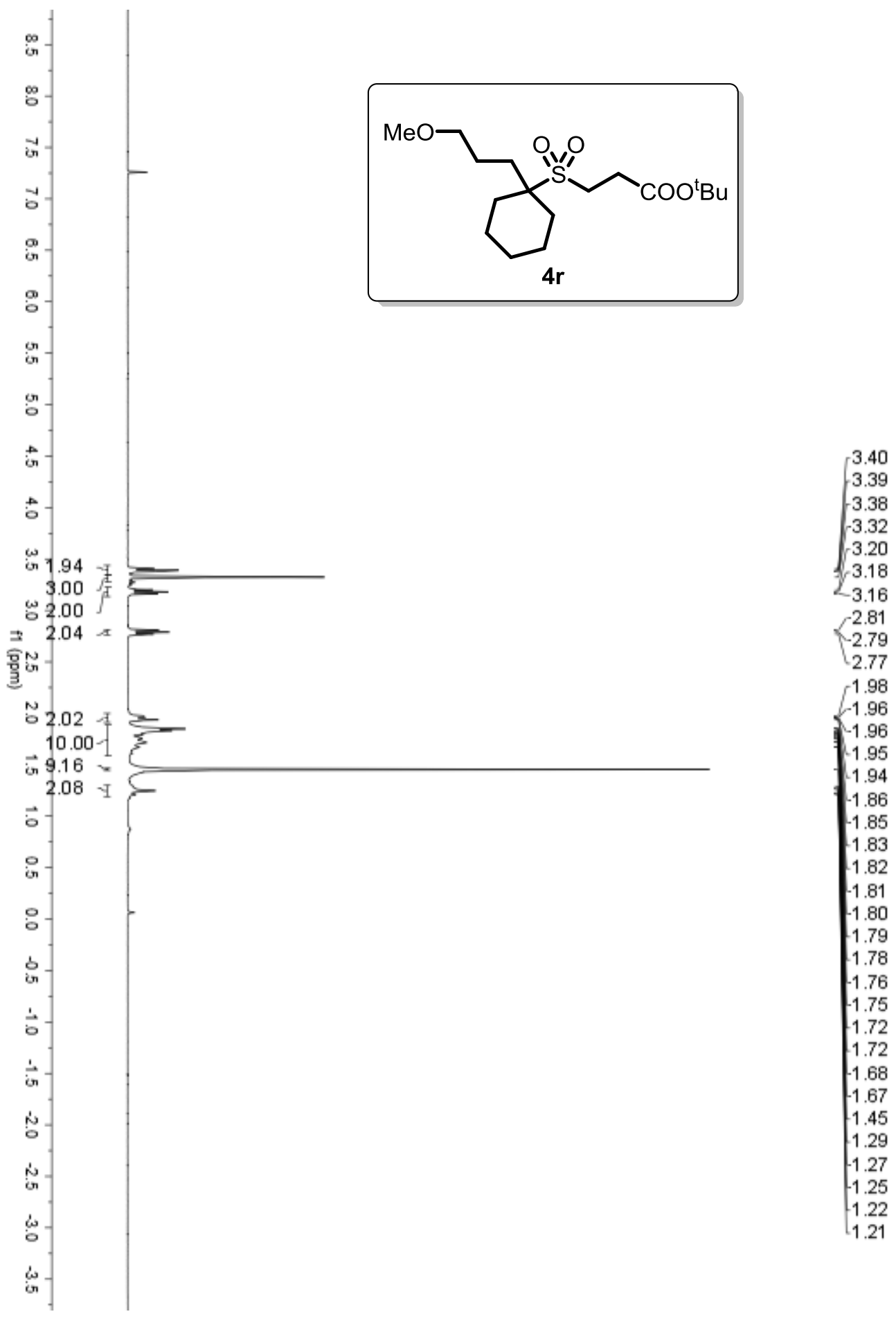


${ }^{13} \mathrm{C}$ NMR $\left(100 \mathrm{MHz}, \mathrm{CDCl}_{3}\right)$

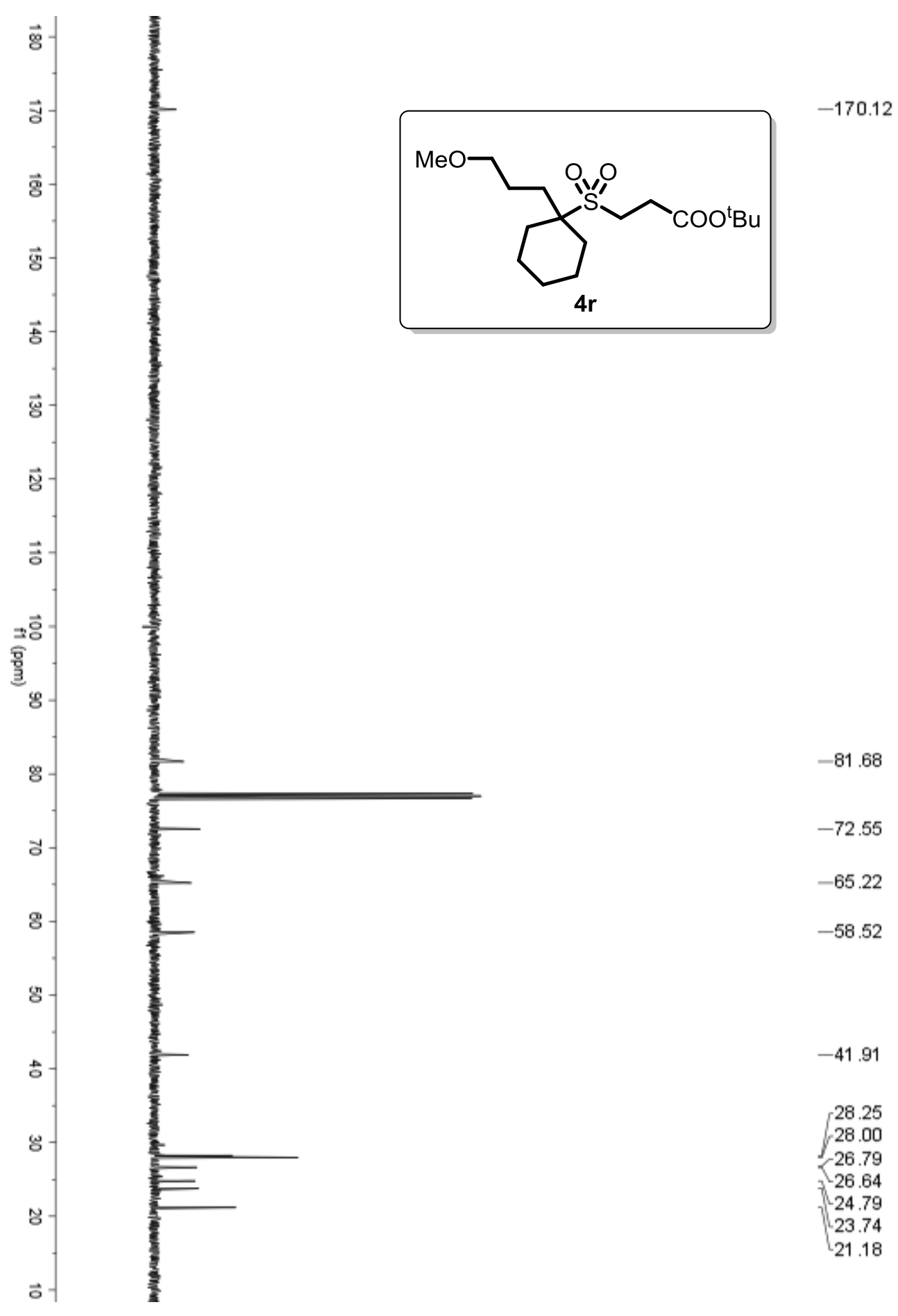


${ }^{1} \mathbf{H}$ NMR $\left(400 \mathrm{MHz}, \mathrm{CDCl}_{3}\right)$

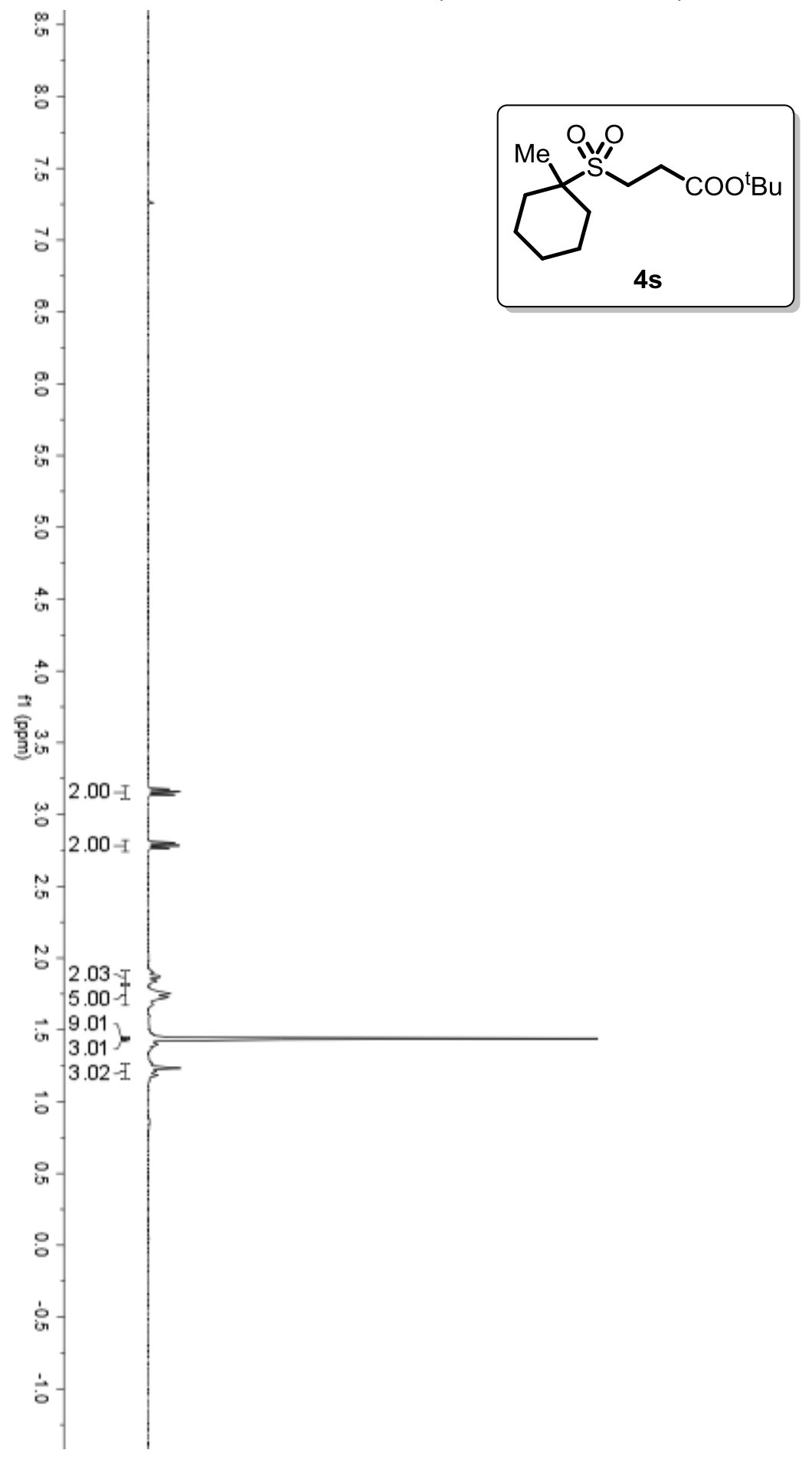


${ }^{13} \mathrm{C}$ NMR $\left(100 \mathrm{MHz}, \mathrm{CDCl}_{3}\right)$

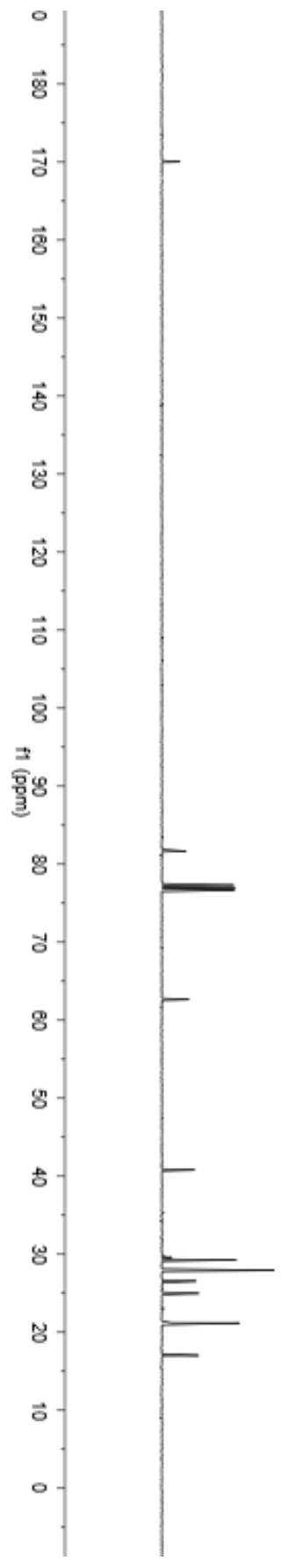

$-170.04$

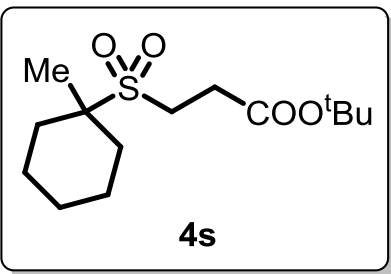

$-81.67$

$-62.66$

$-40.81$

$J^{29.29}$

$\lcm{26.57}$

$-24.99$

21.20

17.04 
${ }^{1} \mathbf{H}$ NMR $\left(400 \mathrm{MHz}, \mathrm{CDCl}_{3}\right)$

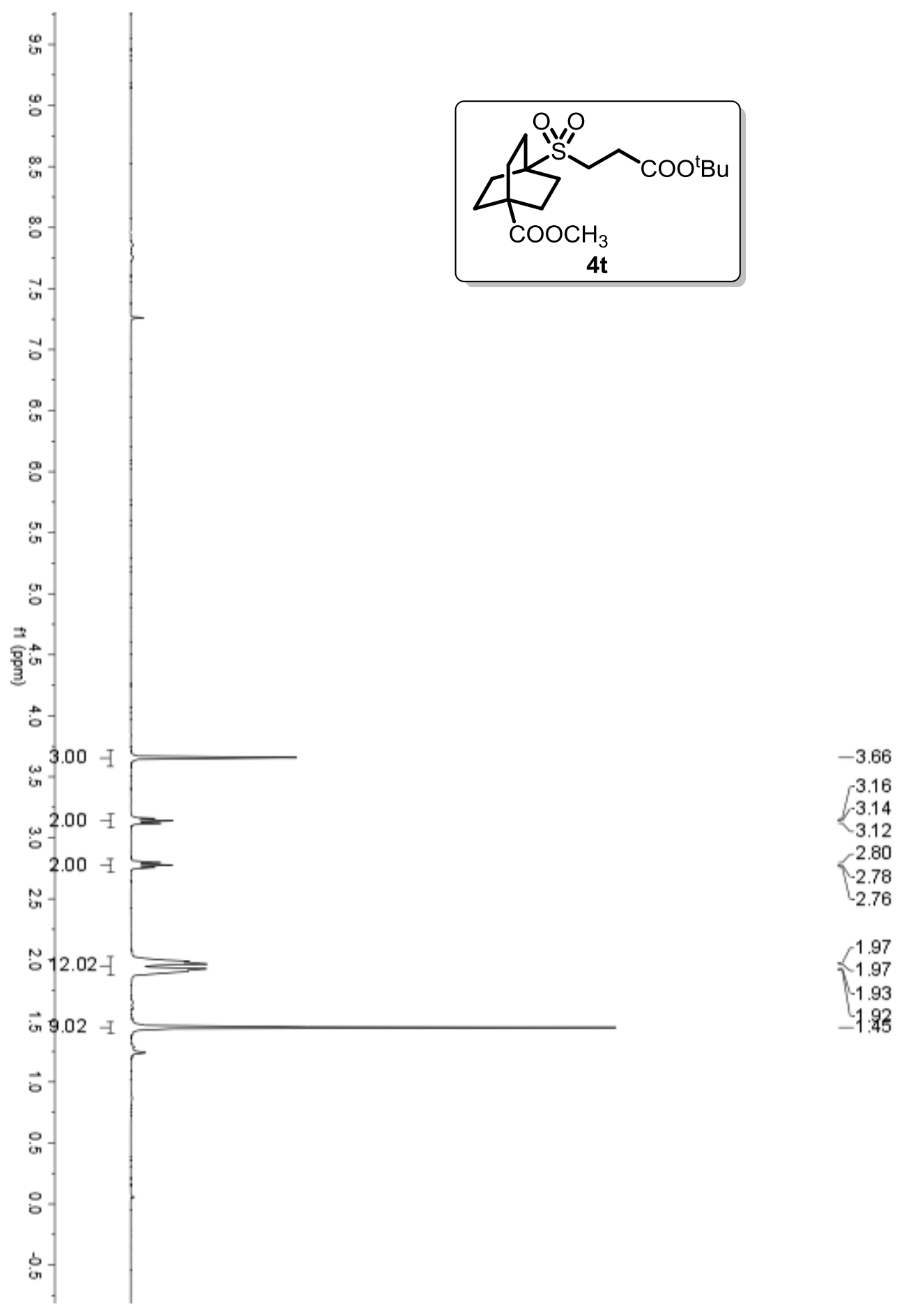


${ }^{13} \mathrm{C}$ NMR $\left(100 \mathrm{MHz}, \mathrm{CDCl}_{3}\right)$

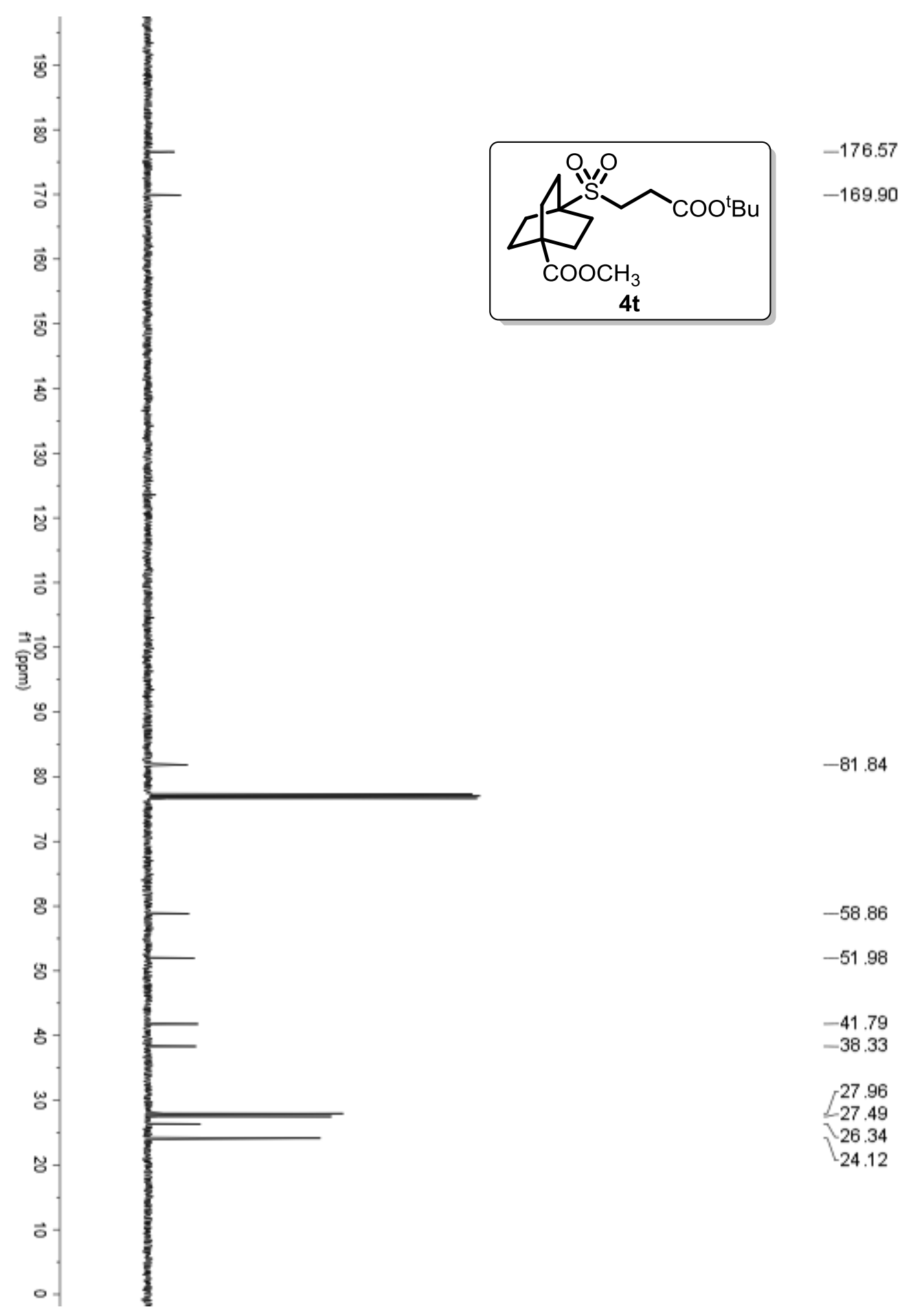


${ }^{1} \mathbf{H}$ NMR $\left(400 \mathrm{MHz}, \mathrm{CDCl}_{3}\right)$

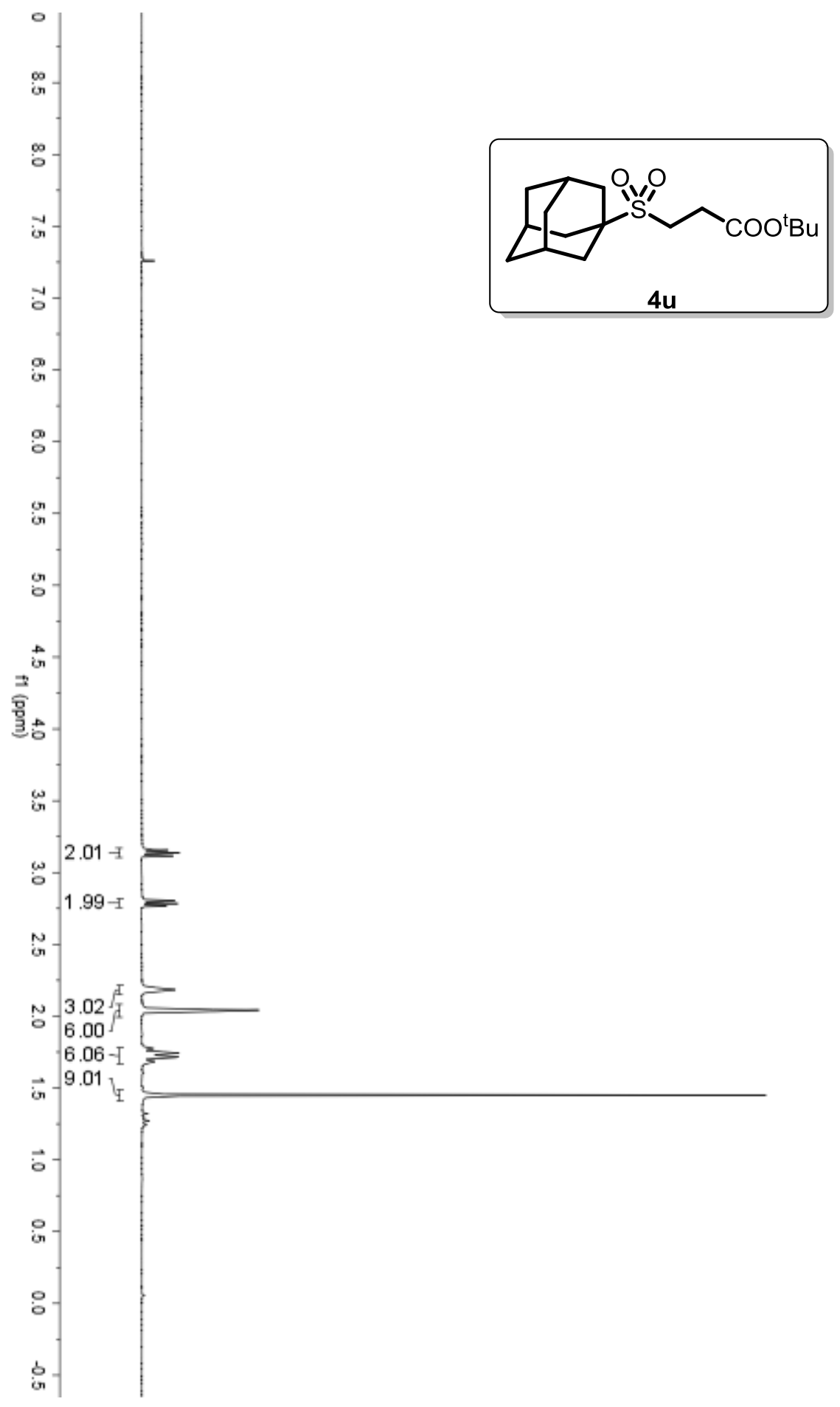


${ }^{13} \mathrm{C}$ NMR $\left(100 \mathrm{MHz}, \mathrm{CDCl}_{3}\right)$
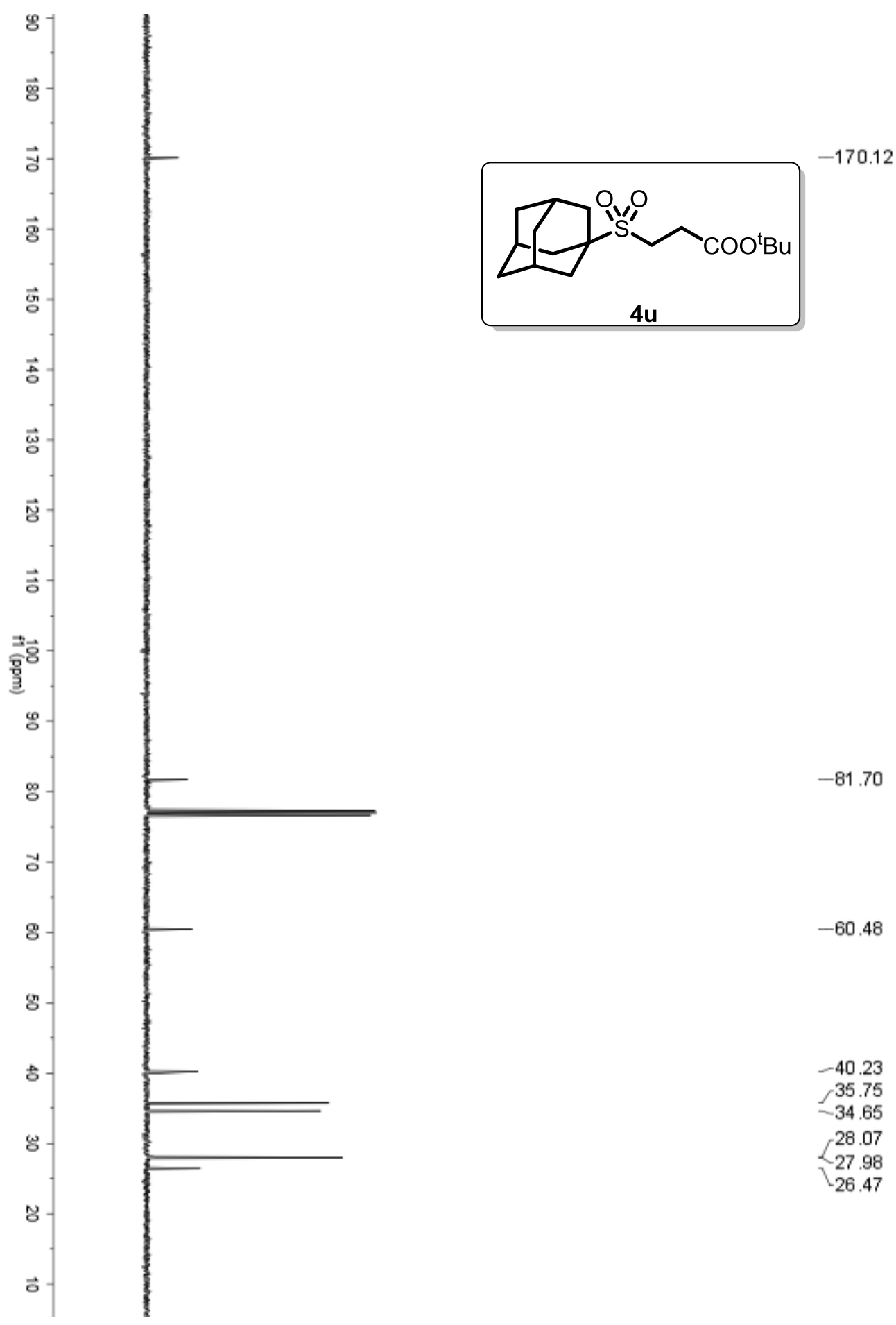

$-81.70$

$-60.48$

$-40.23$

-35.75

$-34.65$

28.07

27.98

-26.47 
${ }^{1} \mathbf{H}$ NMR $\left(400 \mathrm{MHz}, \mathrm{CDCl}_{3}\right)$

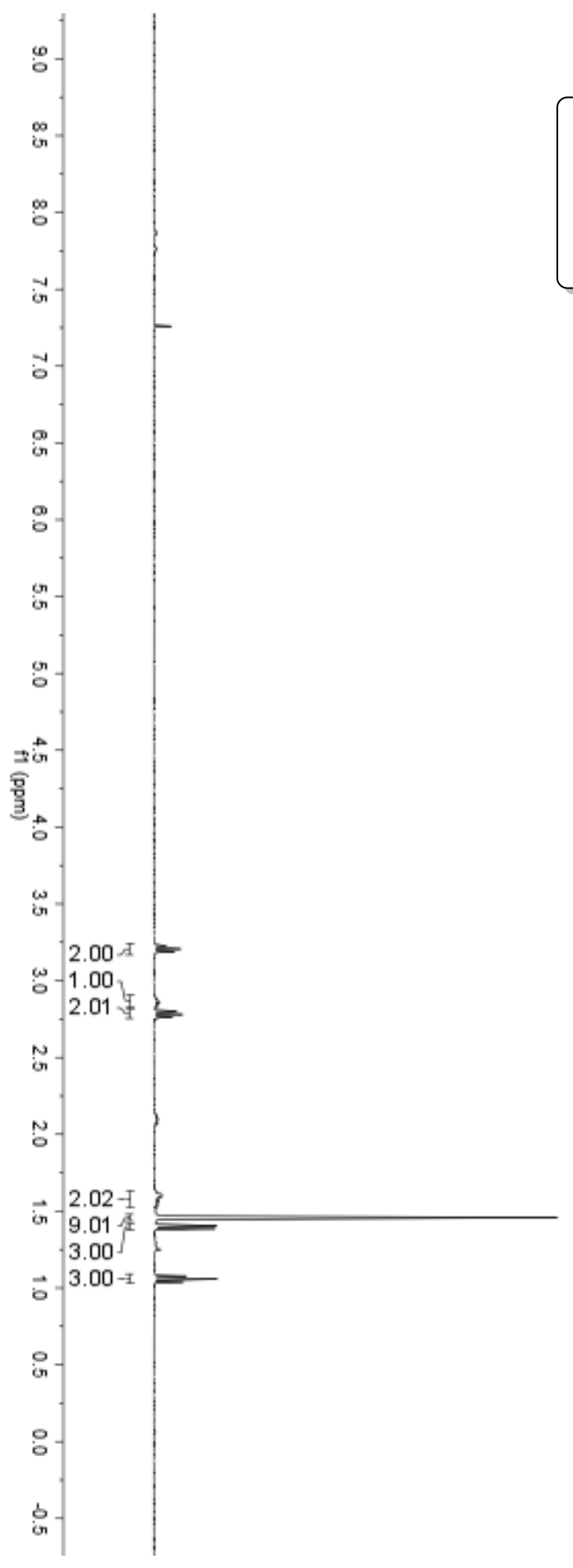


${ }^{13} \mathrm{C}$ NMR $\left(100 \mathrm{MHz}, \mathrm{CDCl}_{3}\right)$
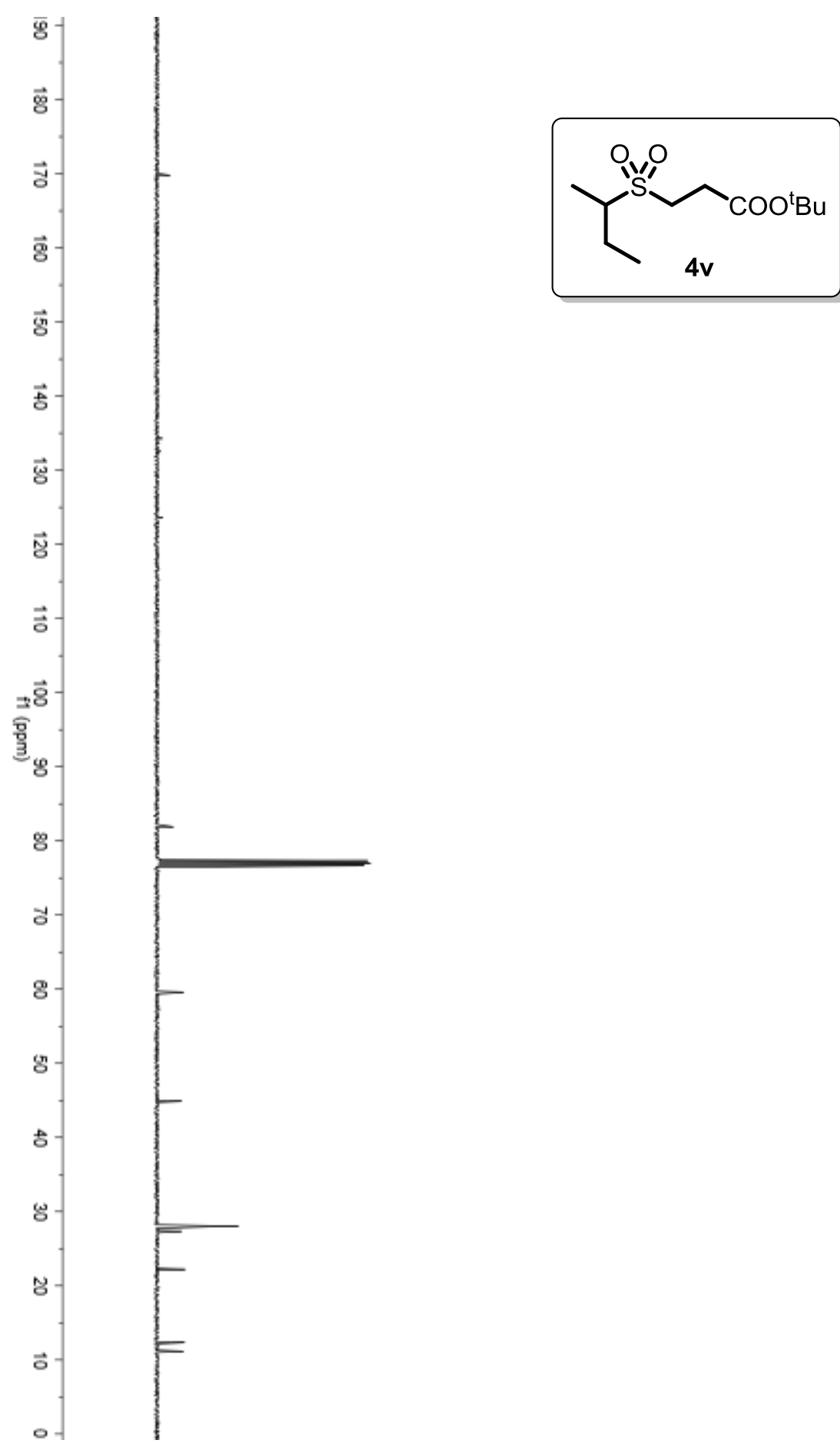

$-169.77$

$-81.89$

$-59.60$

$-44.90$

27.34

$-22.21$

$-12.35$

$-11.15$ 
${ }^{1} \mathbf{H}$ NMR $\left(400 \mathrm{MHz}, \mathrm{CDCl}_{3}\right)$

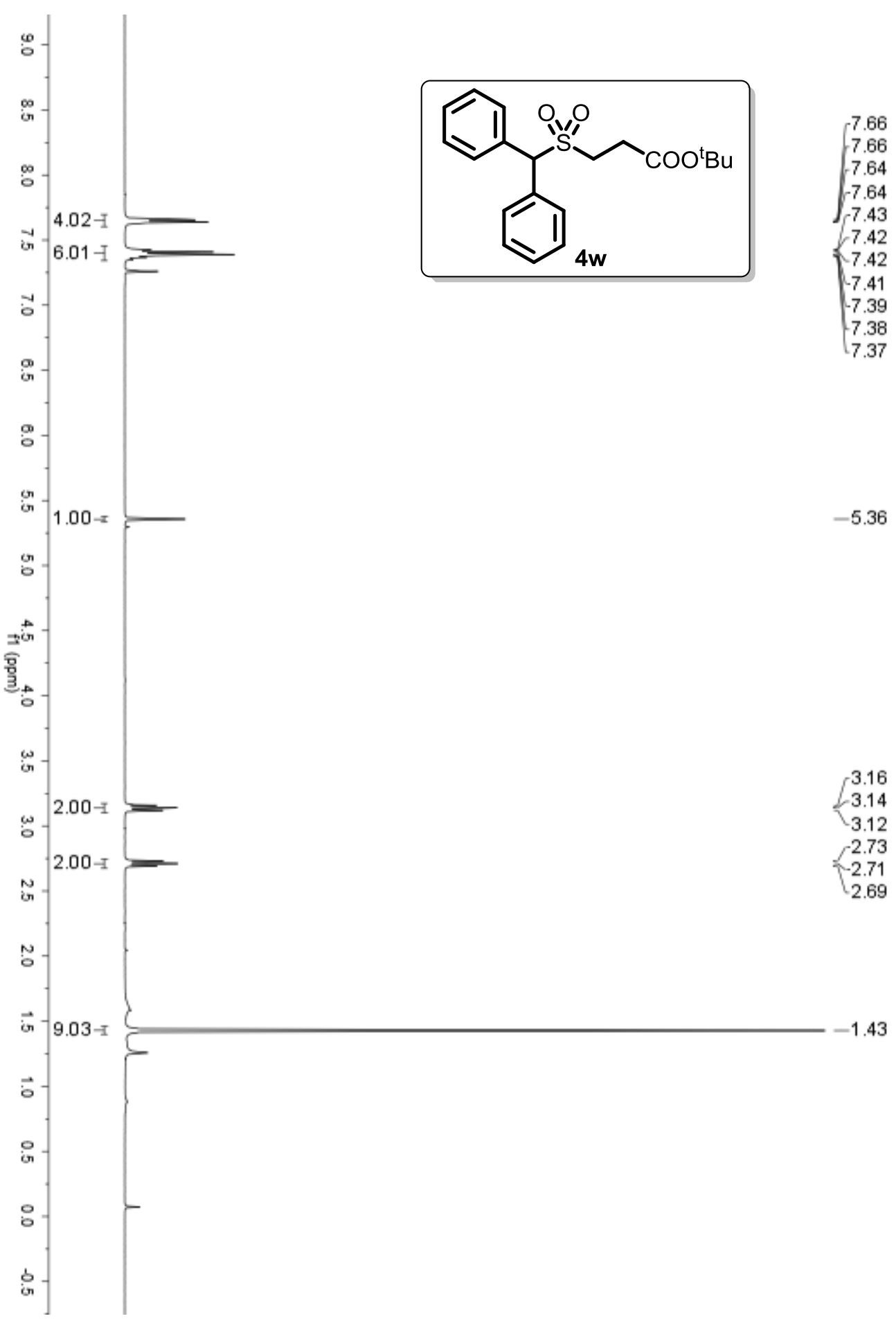


${ }^{13} \mathrm{C}$ NMR $\left(100 \mathrm{MHz}, \mathrm{CDCl}_{3}\right)$

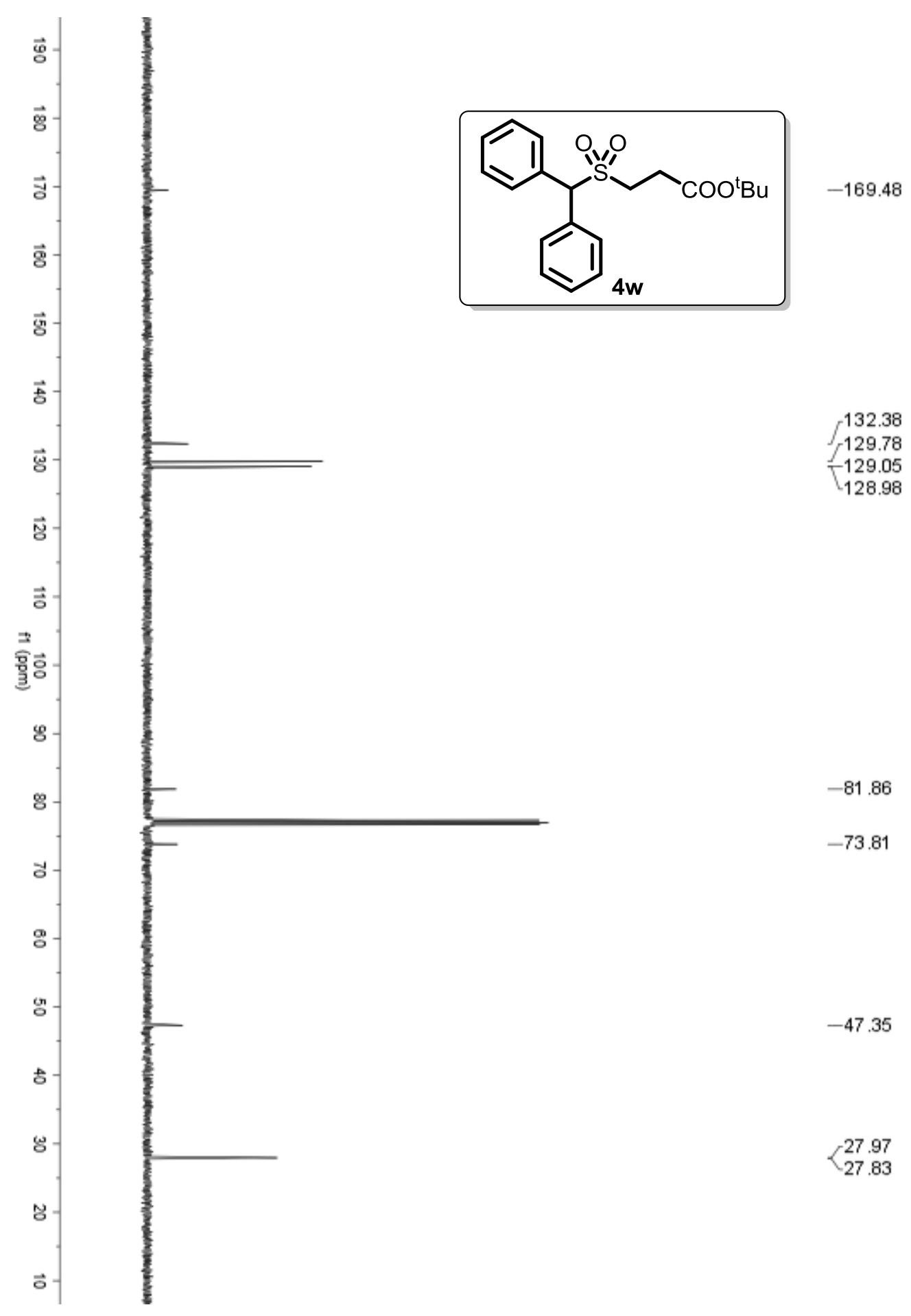


${ }^{1} \mathrm{H}$ NMR $\left(400 \mathrm{MHz}, \mathrm{CDCl}_{3}\right)$

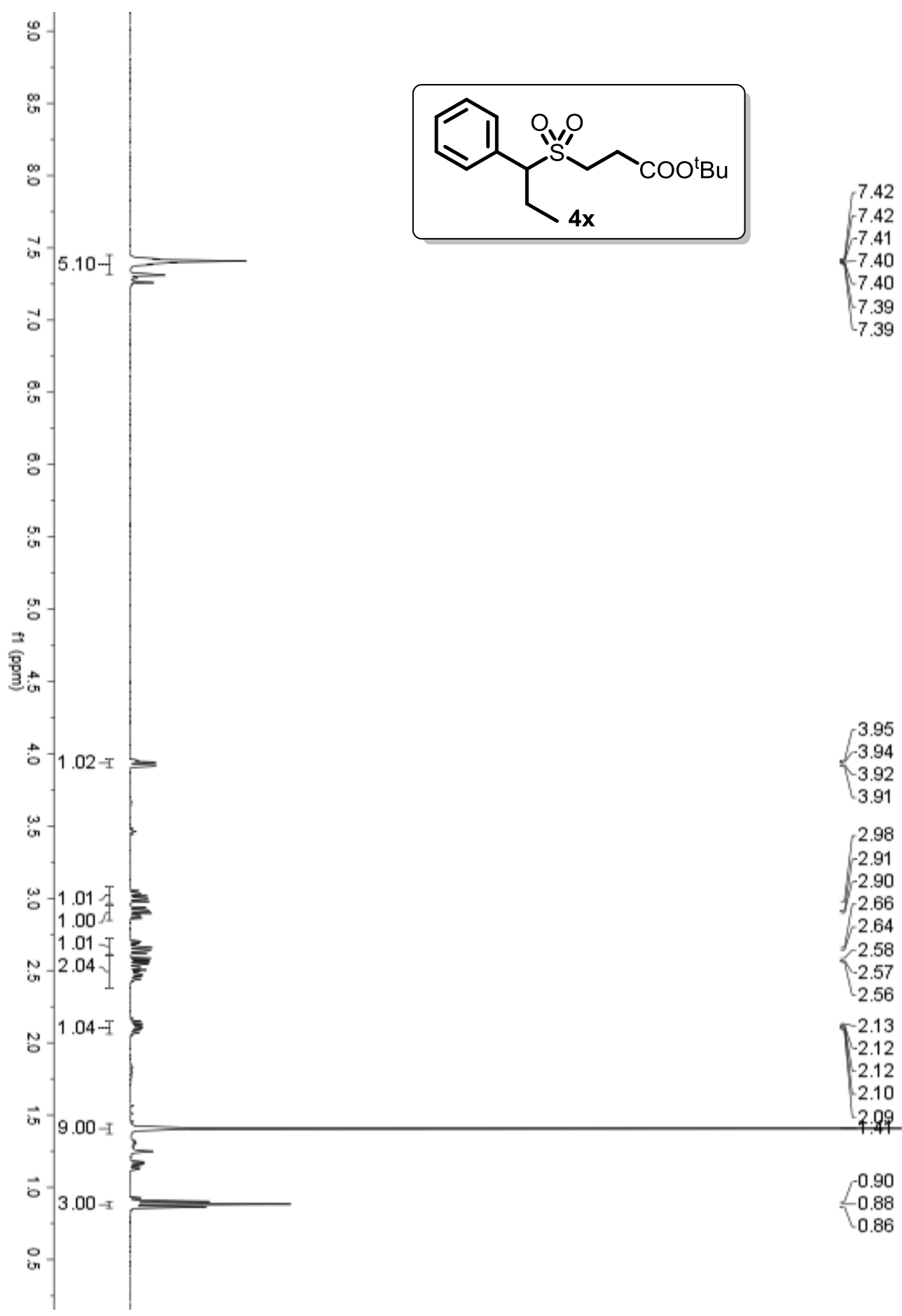


${ }^{13} \mathrm{C}$ NMR $\left(100 \mathrm{MHz}, \mathrm{CDCl}_{3}\right)$
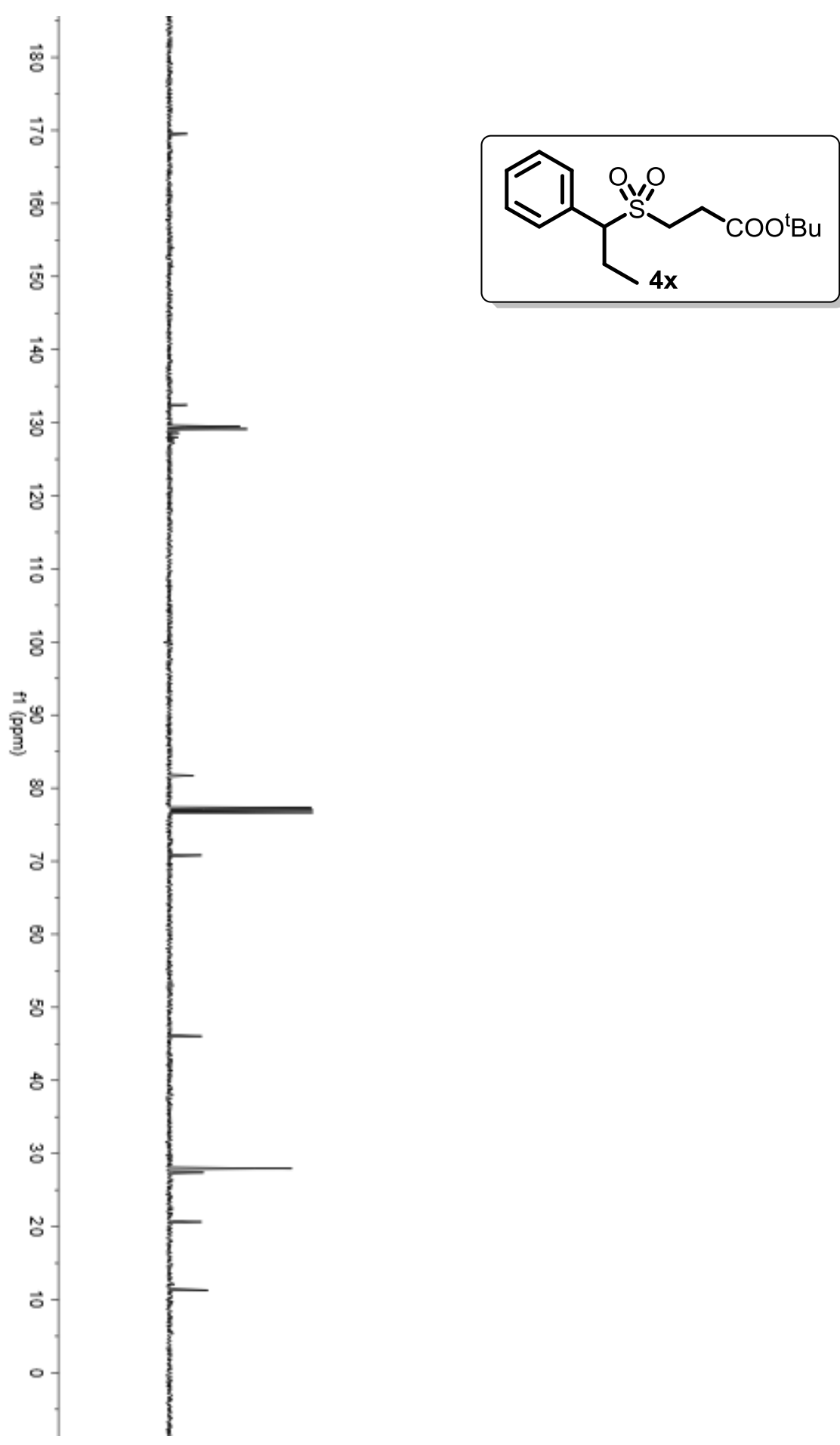

$-169.52$

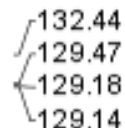

$-81.73$

$-70.81$

$-46.06$

-27.93
27.39

$-20.65$

$-11.32$ 
${ }^{1} \mathbf{H}$ NMR $\left(400 \mathrm{MHz}, \mathrm{CDCl}_{3}\right)$

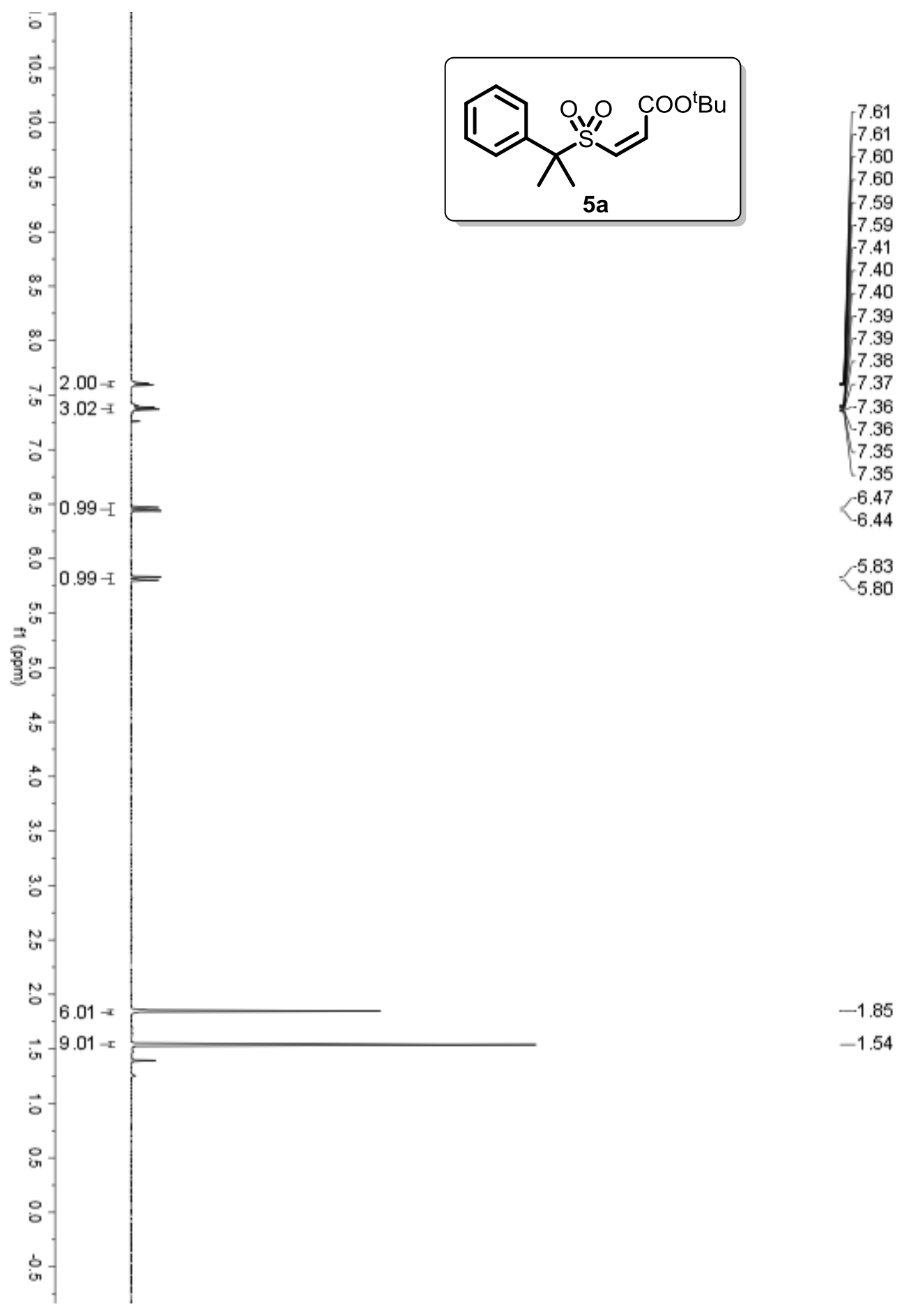


${ }^{13} \mathrm{C}$ NMR $\left(100 \mathrm{MHz}, \mathrm{CDCl}_{3}\right)$

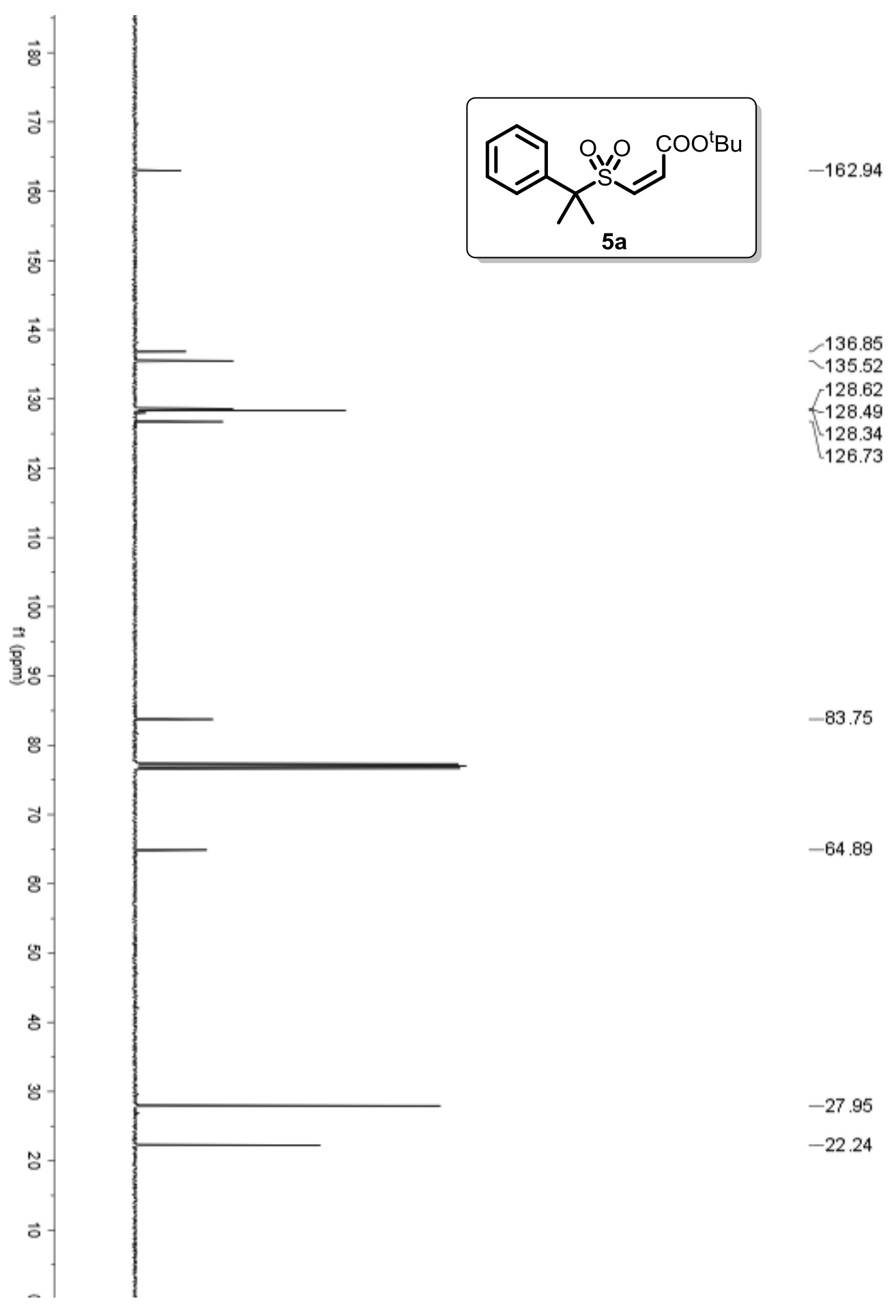


${ }^{1} \mathbf{H}$ NMR $\left(400 \mathrm{MHz}, \mathrm{CDCl}_{3}\right)$

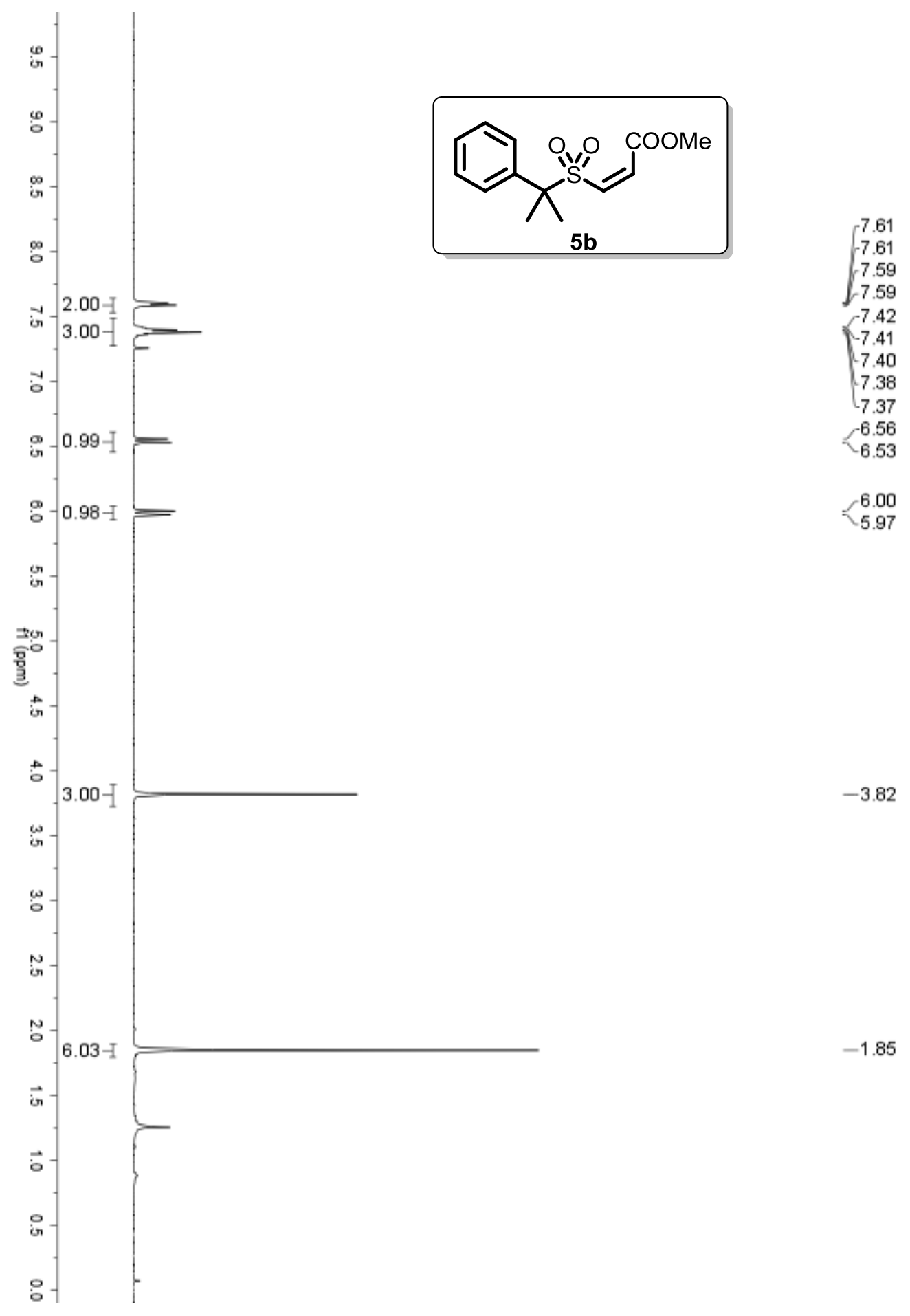


${ }^{13} \mathrm{C}$ NMR $\left(100 \mathrm{MHz}, \mathrm{CDCl}_{3}\right)$
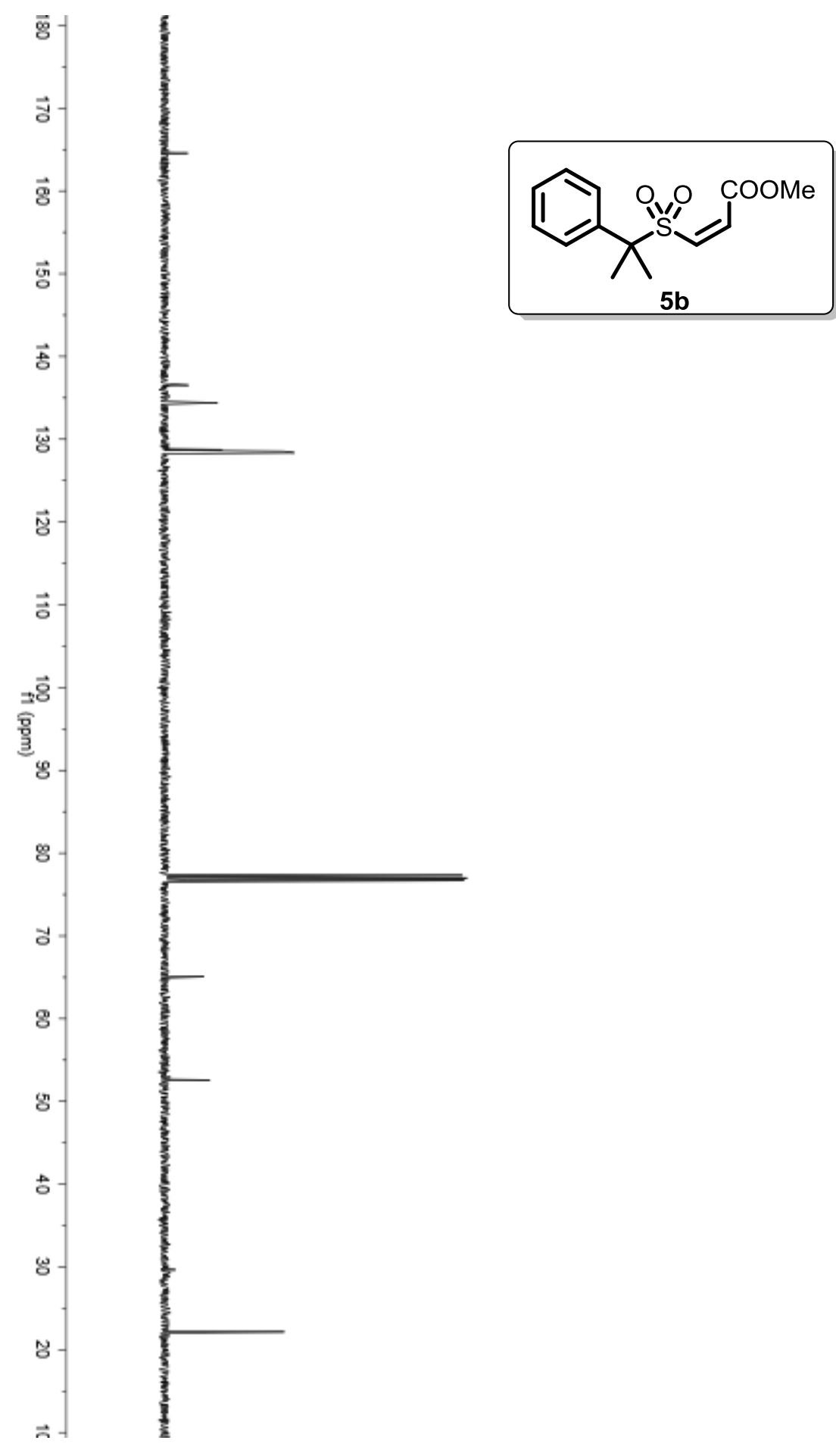

$-164.57$

136.53

134.39

128.74

128.62

128.37

$-65.07$

$-52.58$

$-22.16$ 
${ }^{1} \mathbf{H}$ NMR $\left(400 \mathrm{MHz}, \mathrm{CDCl}_{3}\right)$

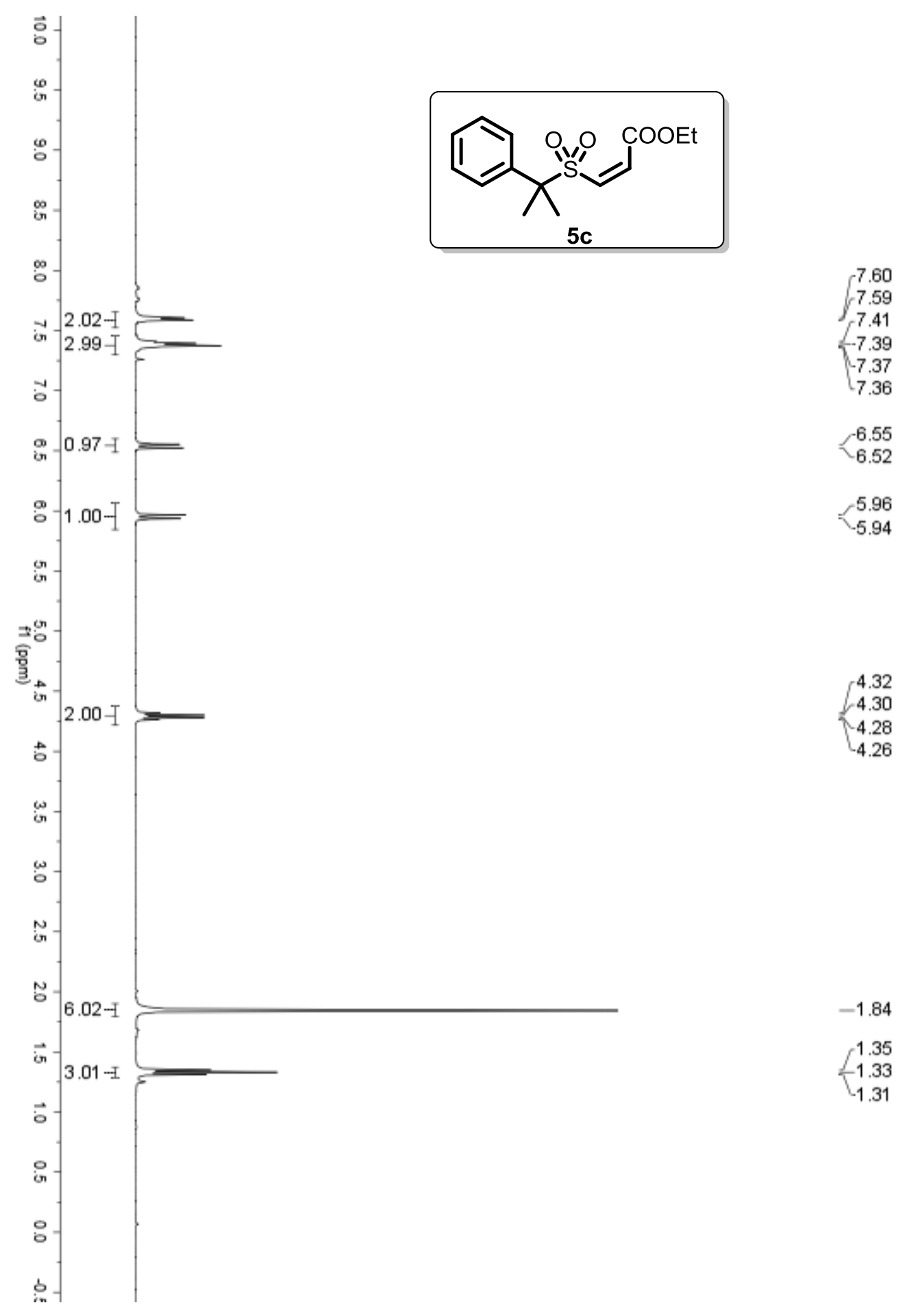


${ }^{13} \mathrm{C}$ NMR $\left(100 \mathrm{MHz}, \mathrm{CDCl}_{3}\right)$
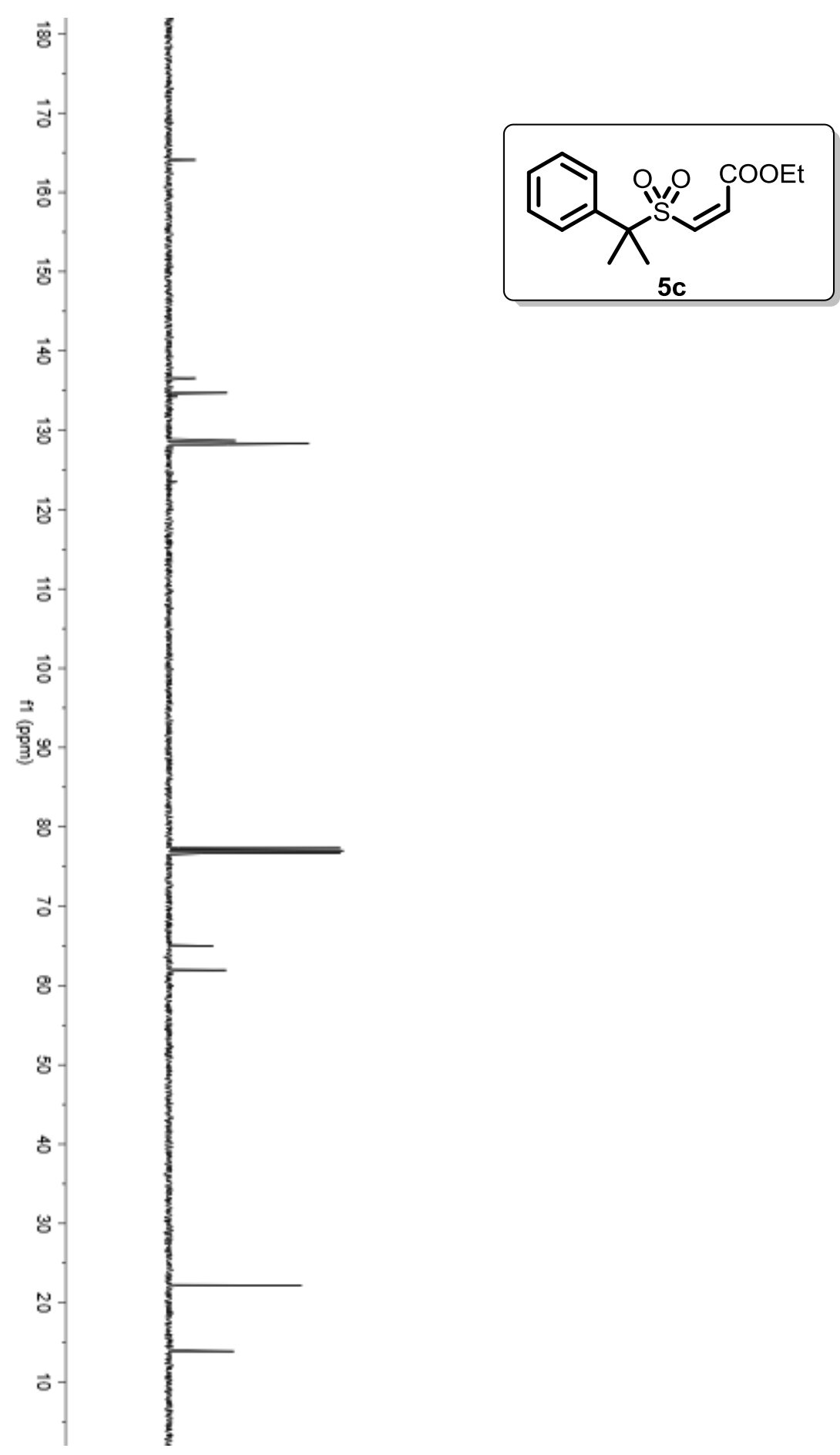

$-164.09$

136.57

134.72

128.68

128.50

-128.34

$-64.97$

$-61.94$

$-22.18$

$-13.89$ 
${ }^{1} \mathbf{H}$ NMR $\left(400 \mathrm{MHz}, \mathrm{CDCl}_{3}\right)$
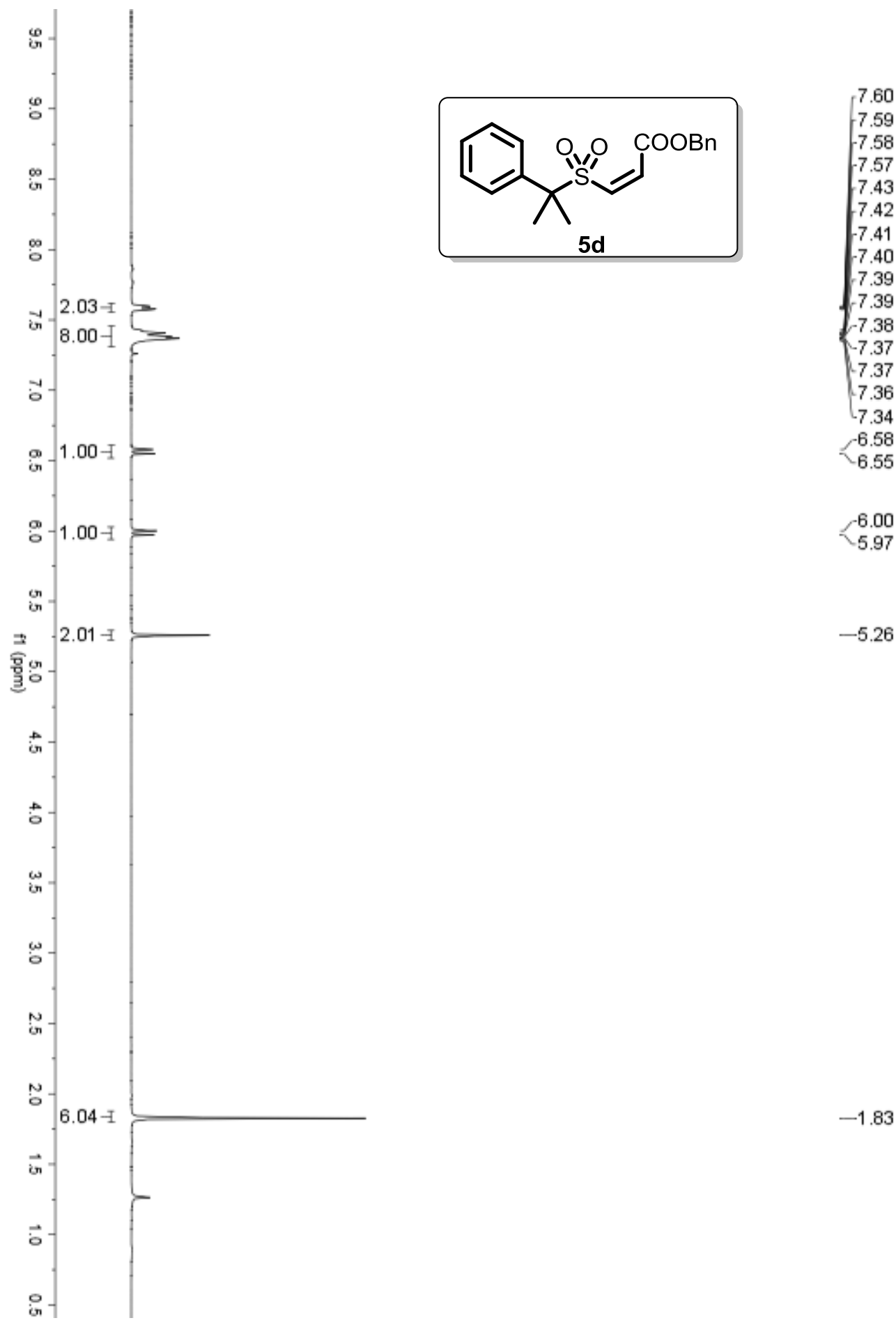

$-5.26$

$-1.83$ 
${ }^{13} \mathrm{C}$ NMR $\left(100 \mathrm{MHz}, \mathrm{CDCl}_{3}\right)$
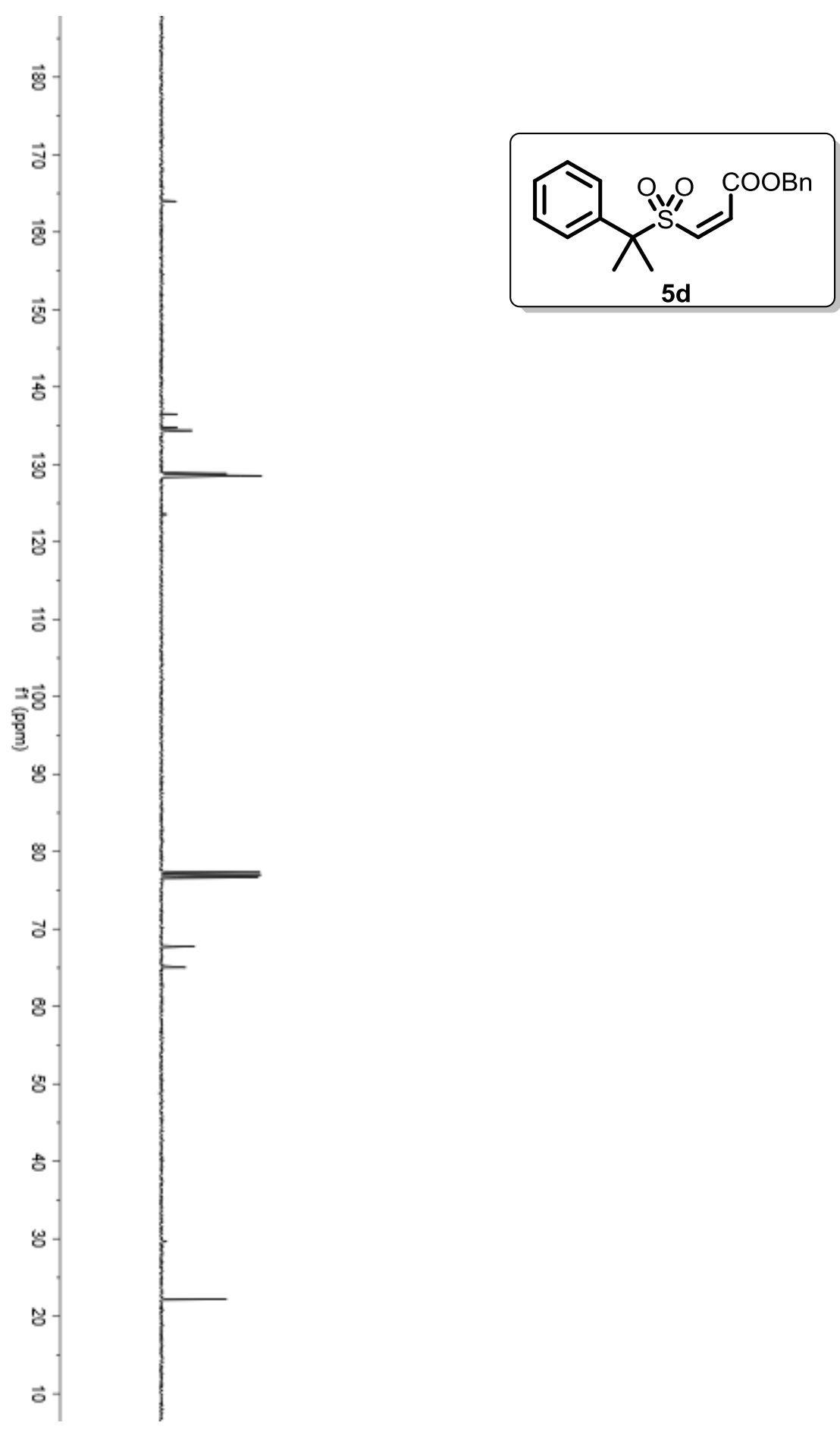

$-163.97$

г136.49

134.78

$-134.37$

134.27

128.81

$-128.71$

-128.62

$-128.53$

$-128.51$

-128.36

$-67.78$

$-65.04$

$-22.20$ 
${ }^{1} \mathrm{H}$ NMR $\left(400 \mathrm{MHz}, \mathrm{CDCl}_{3}\right)$

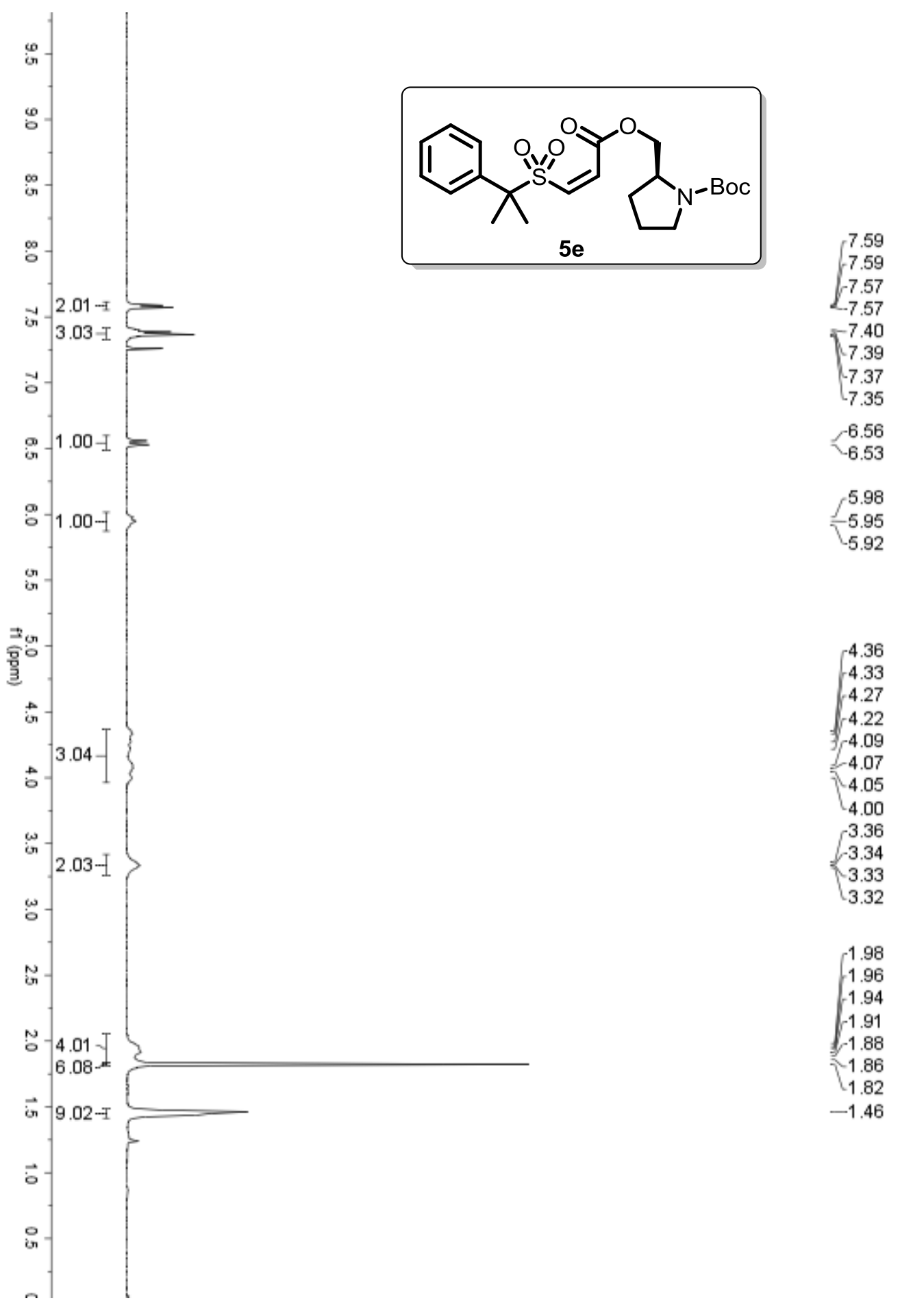


${ }^{13} \mathrm{C}$ NMR $\left(100 \mathrm{MHz}, \mathrm{CDCl}_{3}\right)$

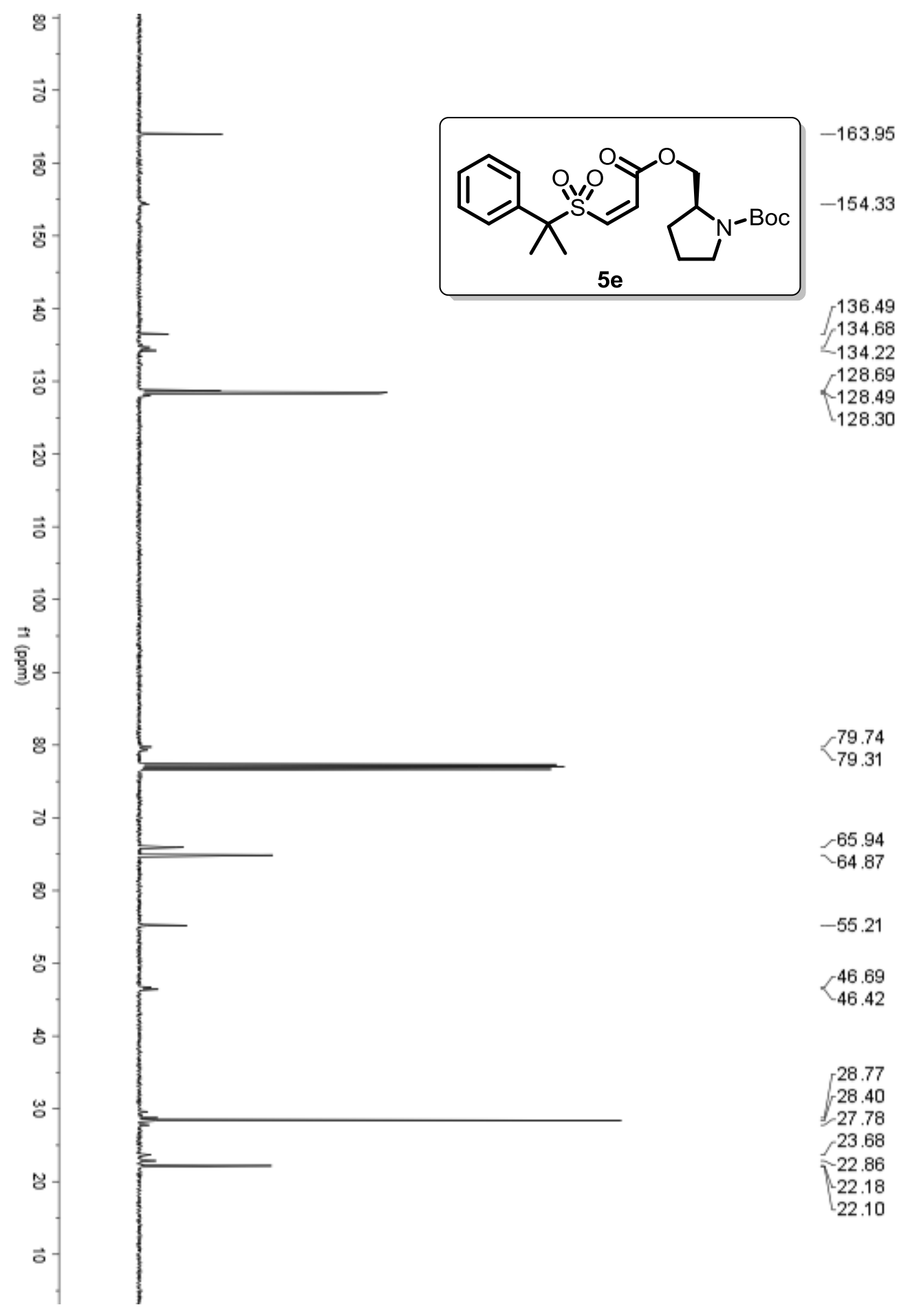


${ }^{1} \mathbf{H}$ NMR $\left(400 \mathrm{MHz}, \mathrm{CDCl}_{3}\right)$

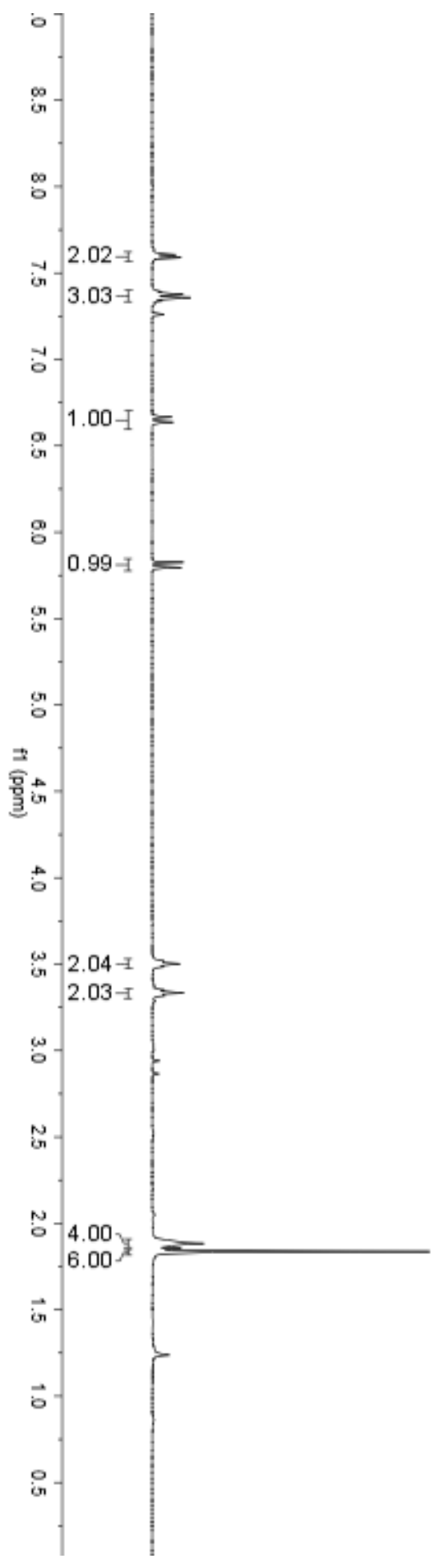


${ }^{13} \mathrm{C}$ NMR $\left(100 \mathrm{MHz}, \mathrm{CDCl}_{3}\right)$

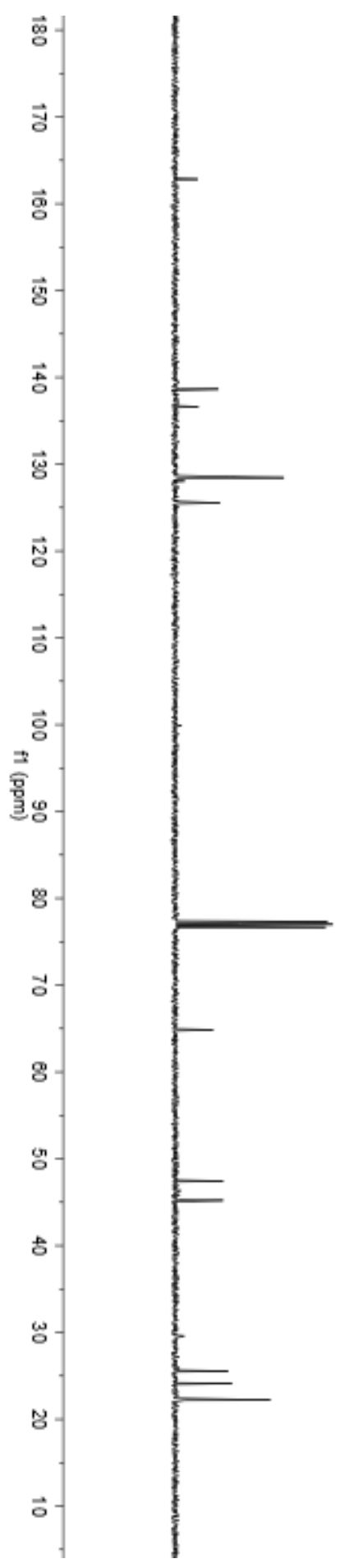

$-162.83$

$-138.64$

$-136.61$

128.57

$-128.45$

$-128.39$

125.54

$-64.85$

$-47.43$

$-45.21$

25.54

$-24.12$

$\checkmark 22.21$ 
${ }^{1} \mathbf{H}$ NMR $\left(400 \mathrm{MHz}, \mathrm{CDCl}_{3}\right)$

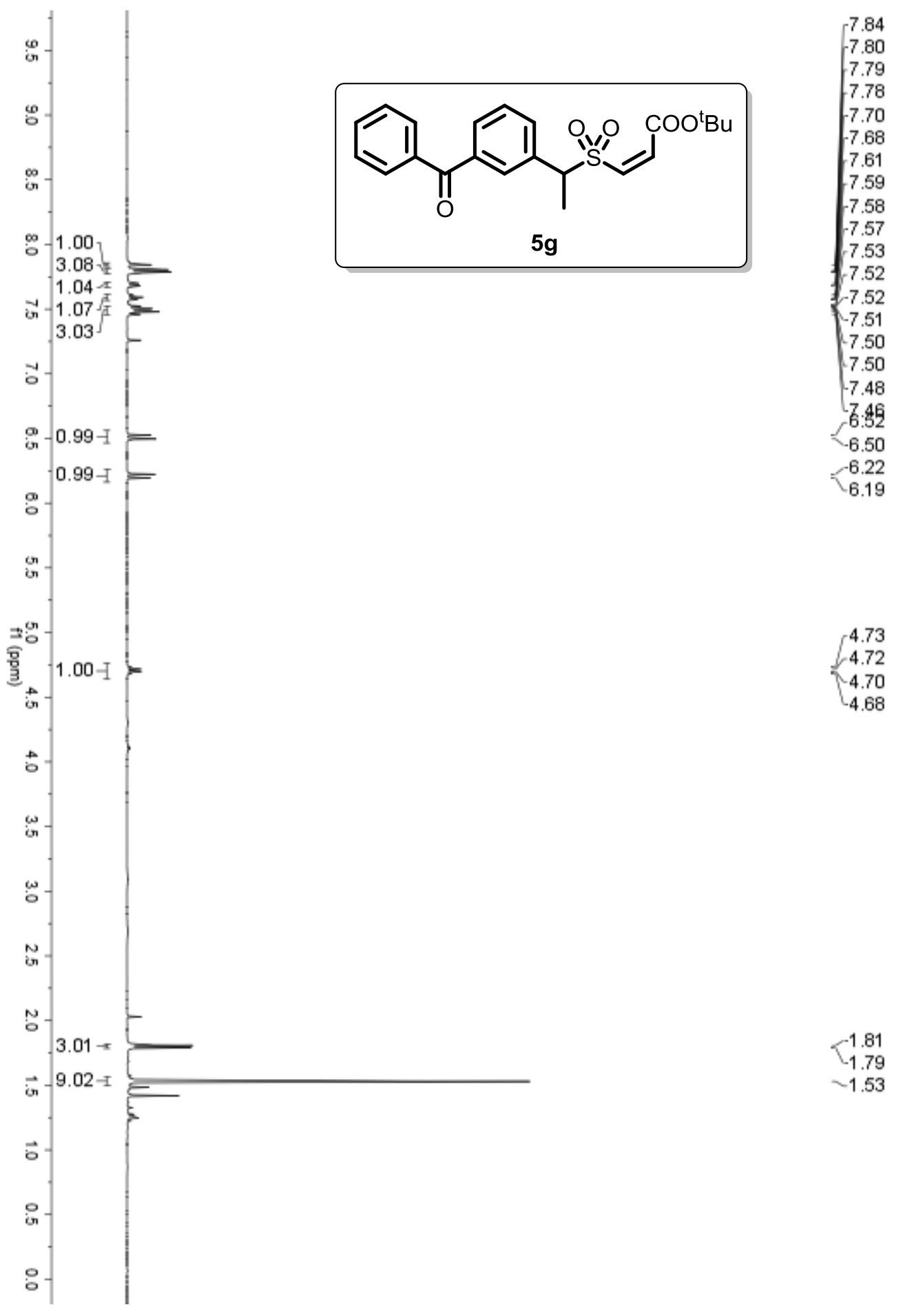


${ }^{13} \mathrm{C}$ NMR $\left(100 \mathrm{MHz}, \mathrm{CDCl}_{3}\right)$

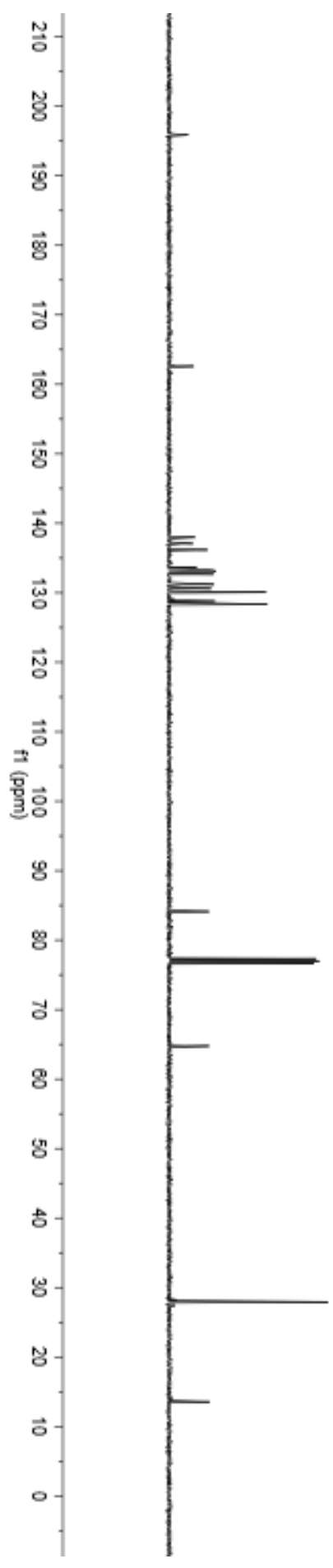

$-195.91$

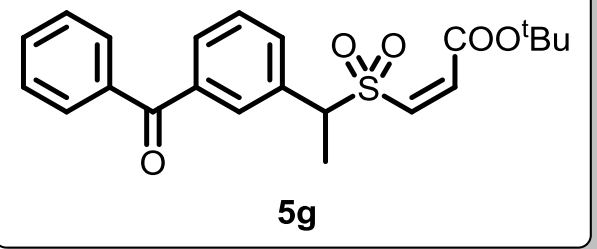

$-162.61$

137.98

$-137.06$

136.18

133.65

133.31

$-133.12$

$-132.68$

131.19

$-130.61$

$-130.07$

$-128.80$

128.34

$-84.12$

$-64.76$

$-27.93$

$-13.56$ 
${ }^{1} \mathbf{H}$ NMR $\left(400 \mathrm{MHz}, \mathrm{CDCl}_{3}\right)$

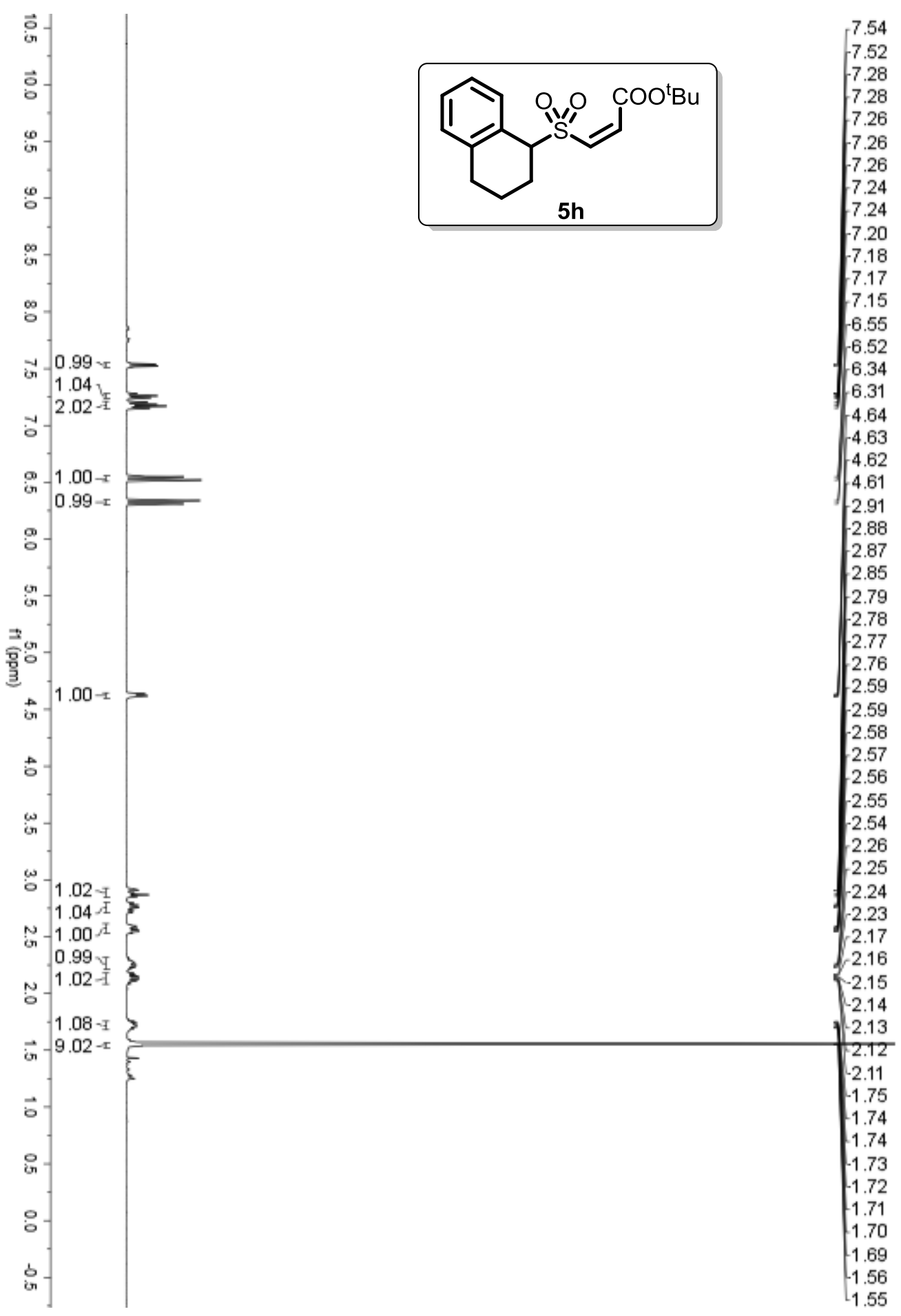


${ }^{13} \mathrm{C}$ NMR $\left(100 \mathrm{MHz}, \mathrm{CDCl}_{3}\right)$

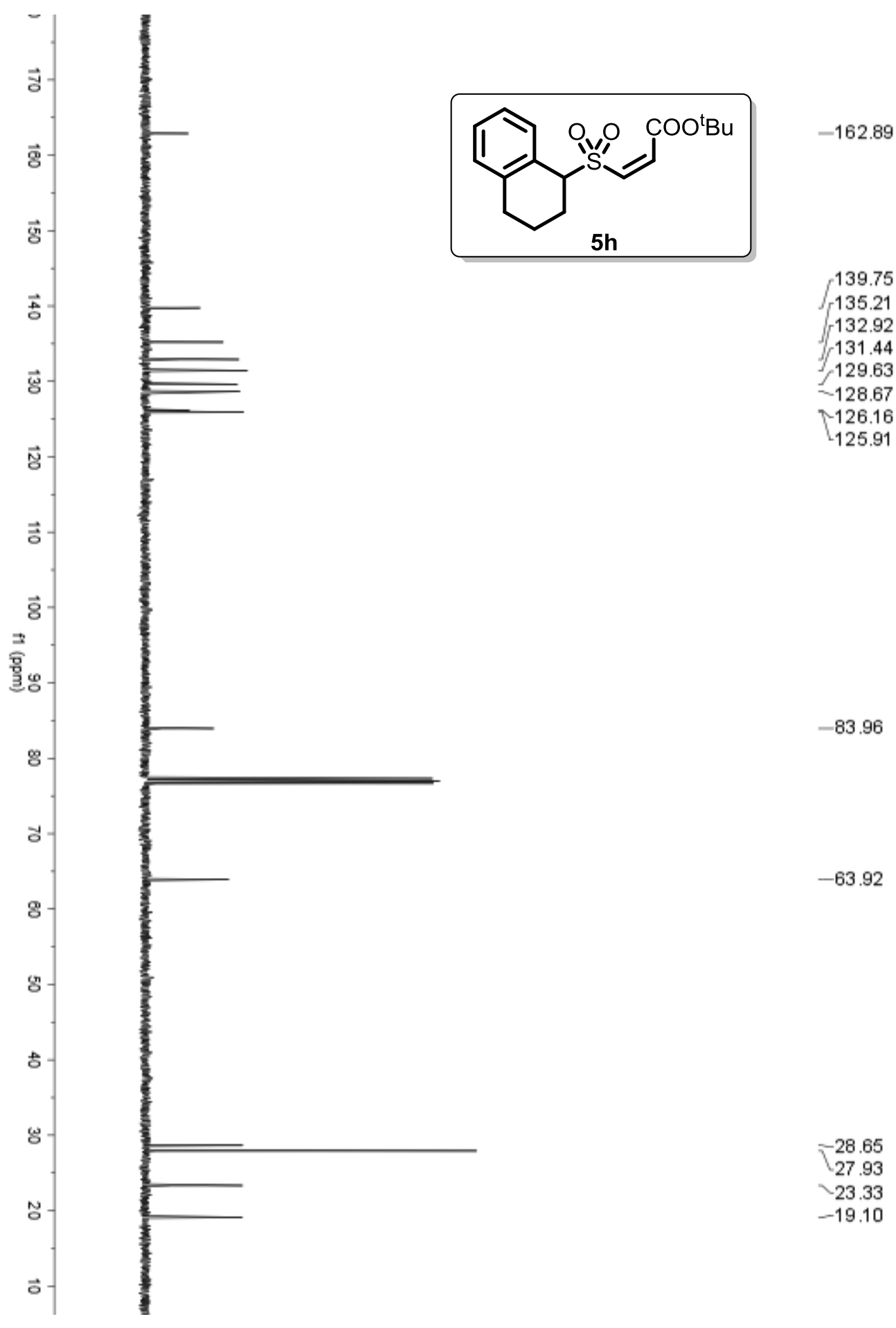


${ }^{1} \mathbf{H}$ NMR $\left(400 \mathrm{MHz}, \mathrm{CDCl}_{3}\right)$

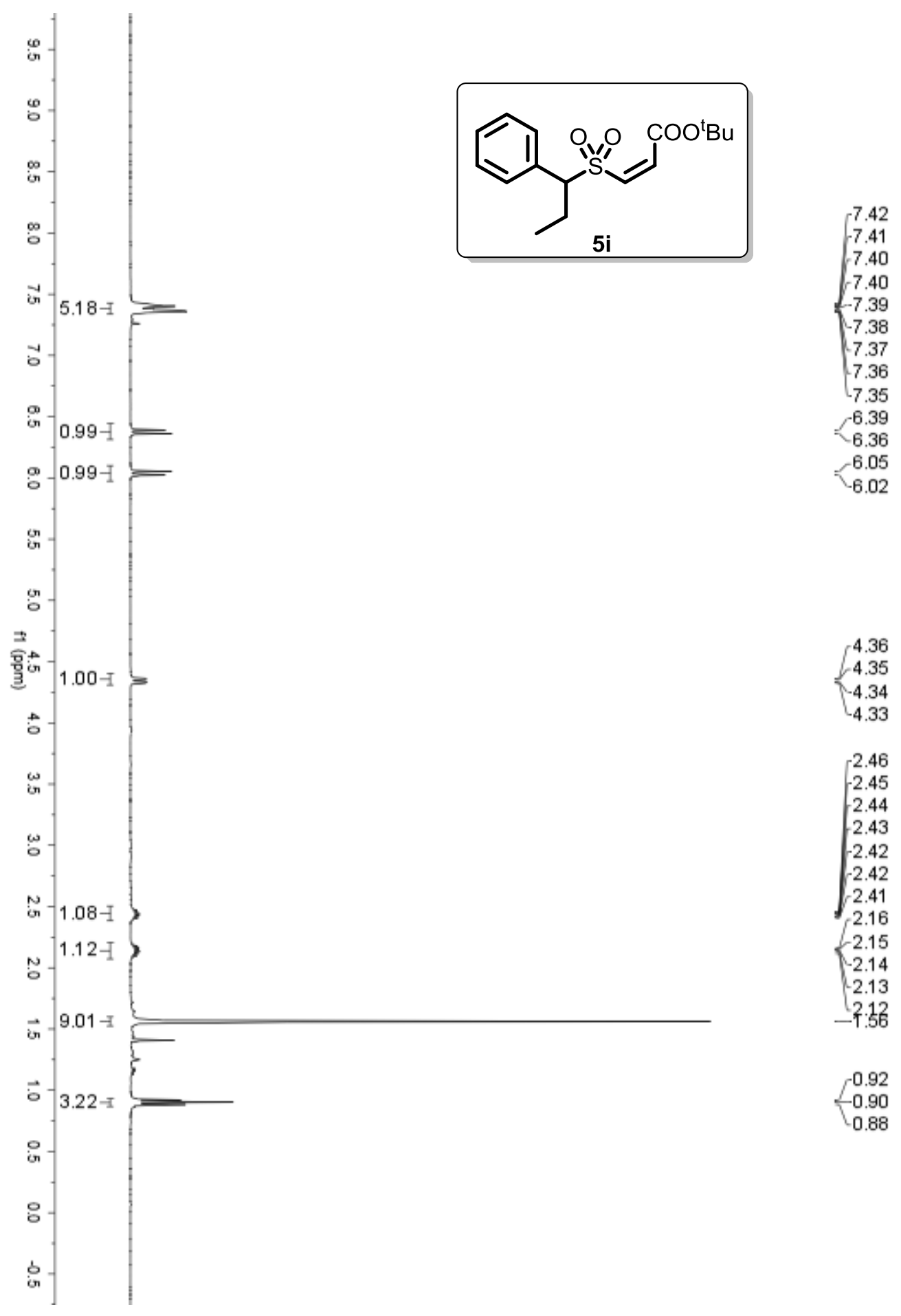


${ }^{13} \mathrm{C}$ NMR $\left(100 \mathrm{MHz}, \mathrm{CDCl}_{3}\right)$

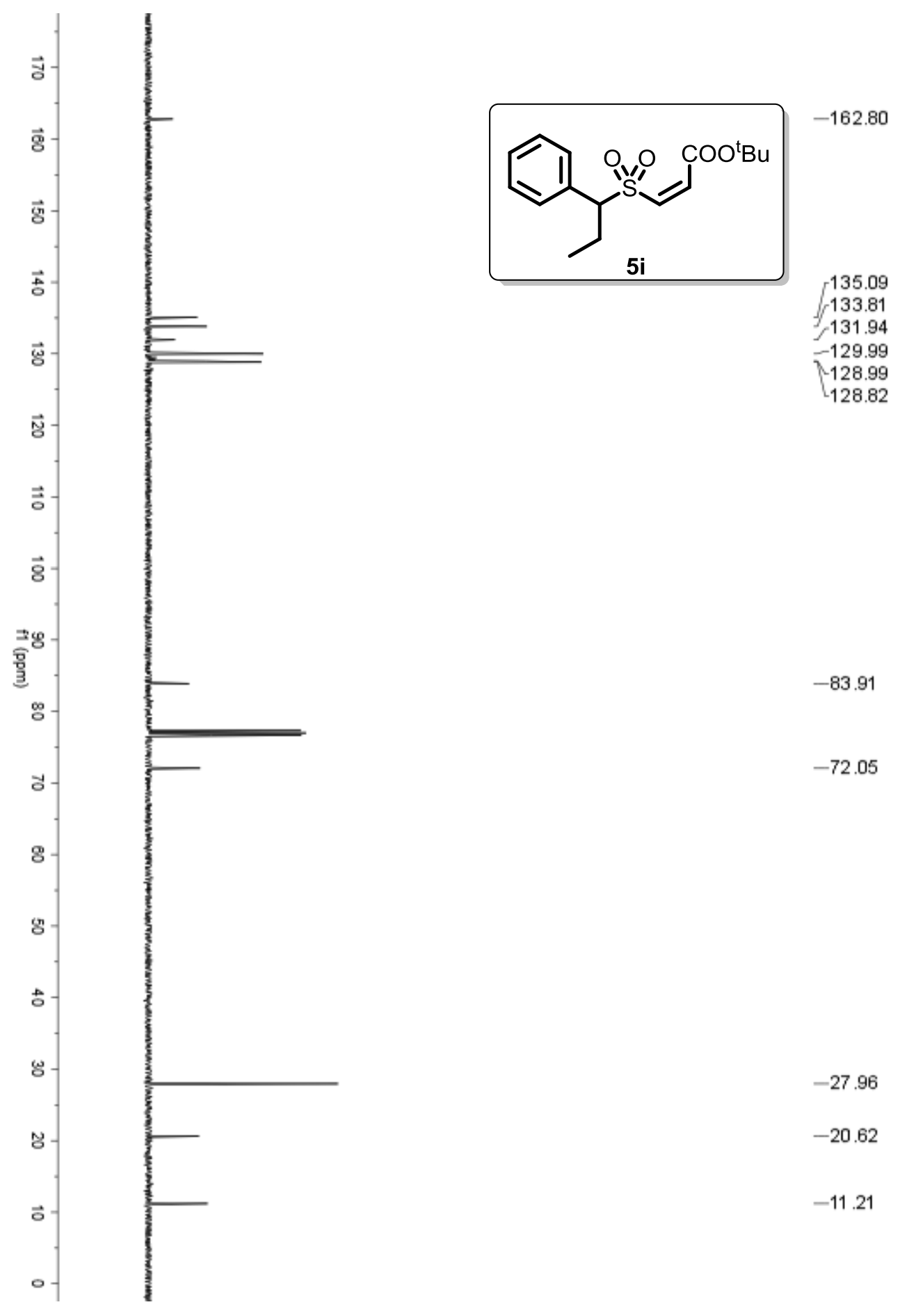


${ }^{1} \mathbf{H}$ NMR $\left(400 \mathrm{MHz}, \mathrm{CDCl}_{3}\right)$

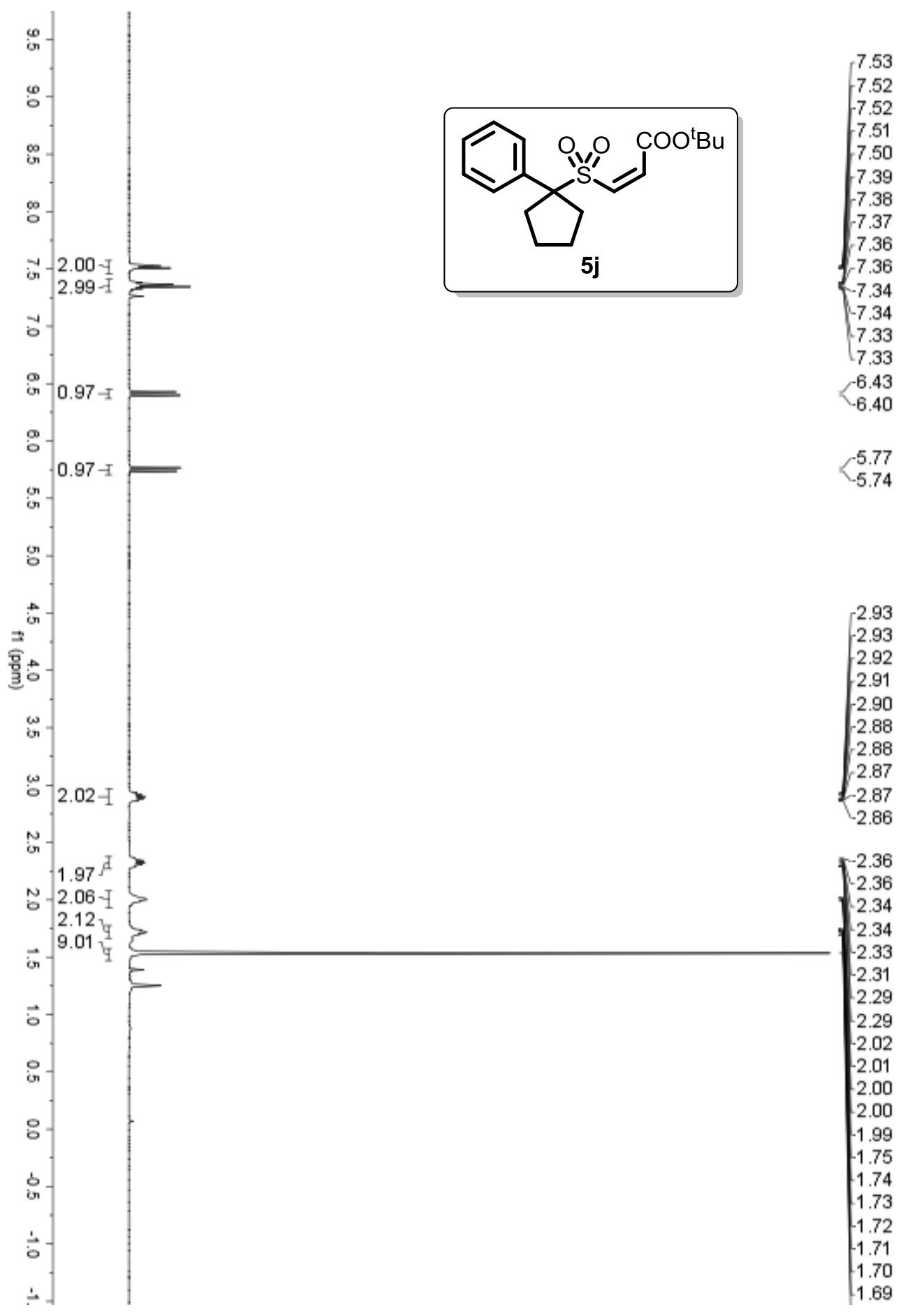


${ }^{13} \mathrm{C}$ NMR $\left(100 \mathrm{MHz}, \mathrm{CDCl}_{3}\right)$

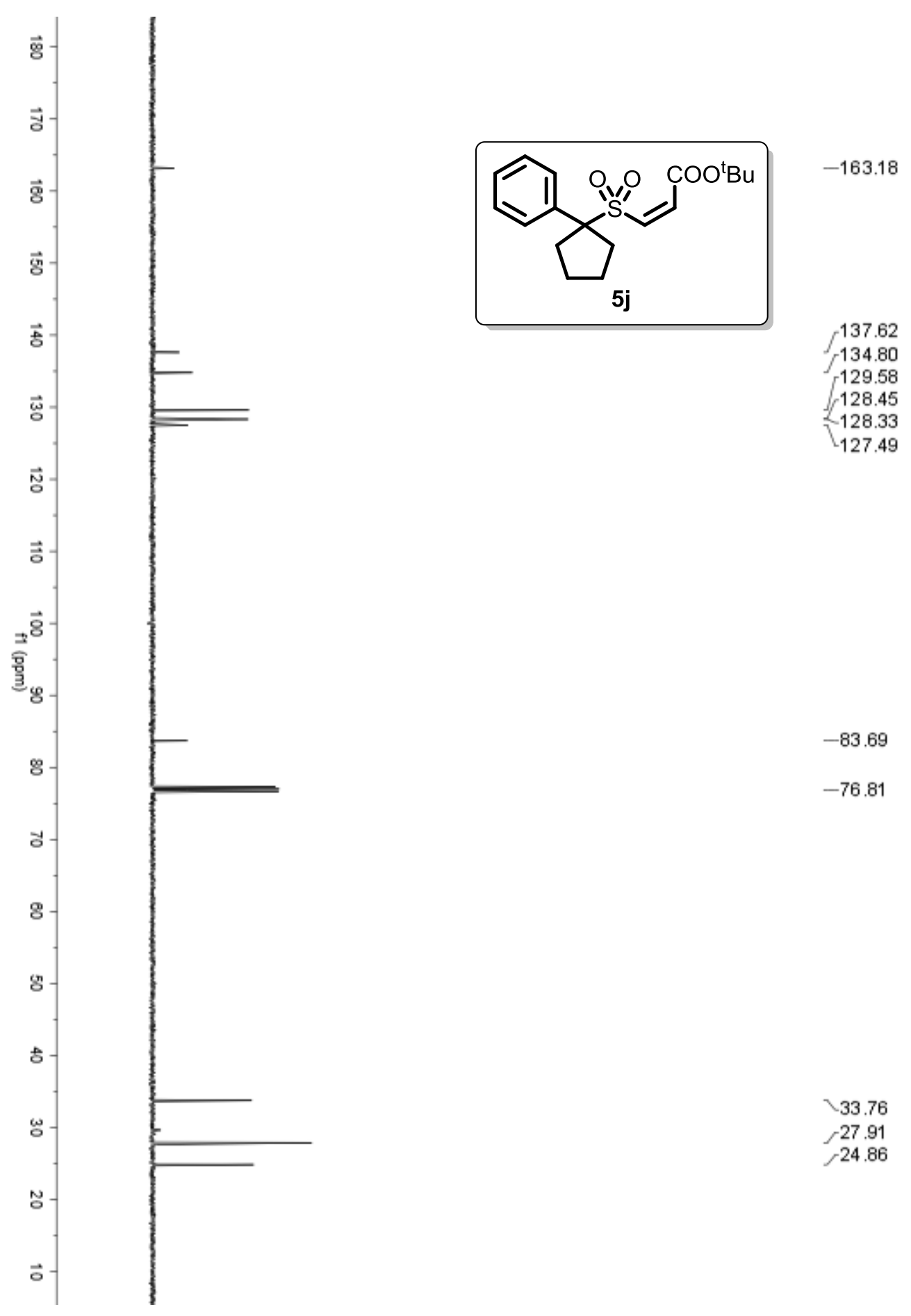


${ }^{1} \mathbf{H}$ NMR $\left(400 \mathrm{MHz}, \mathrm{CDCl}_{3}\right)$

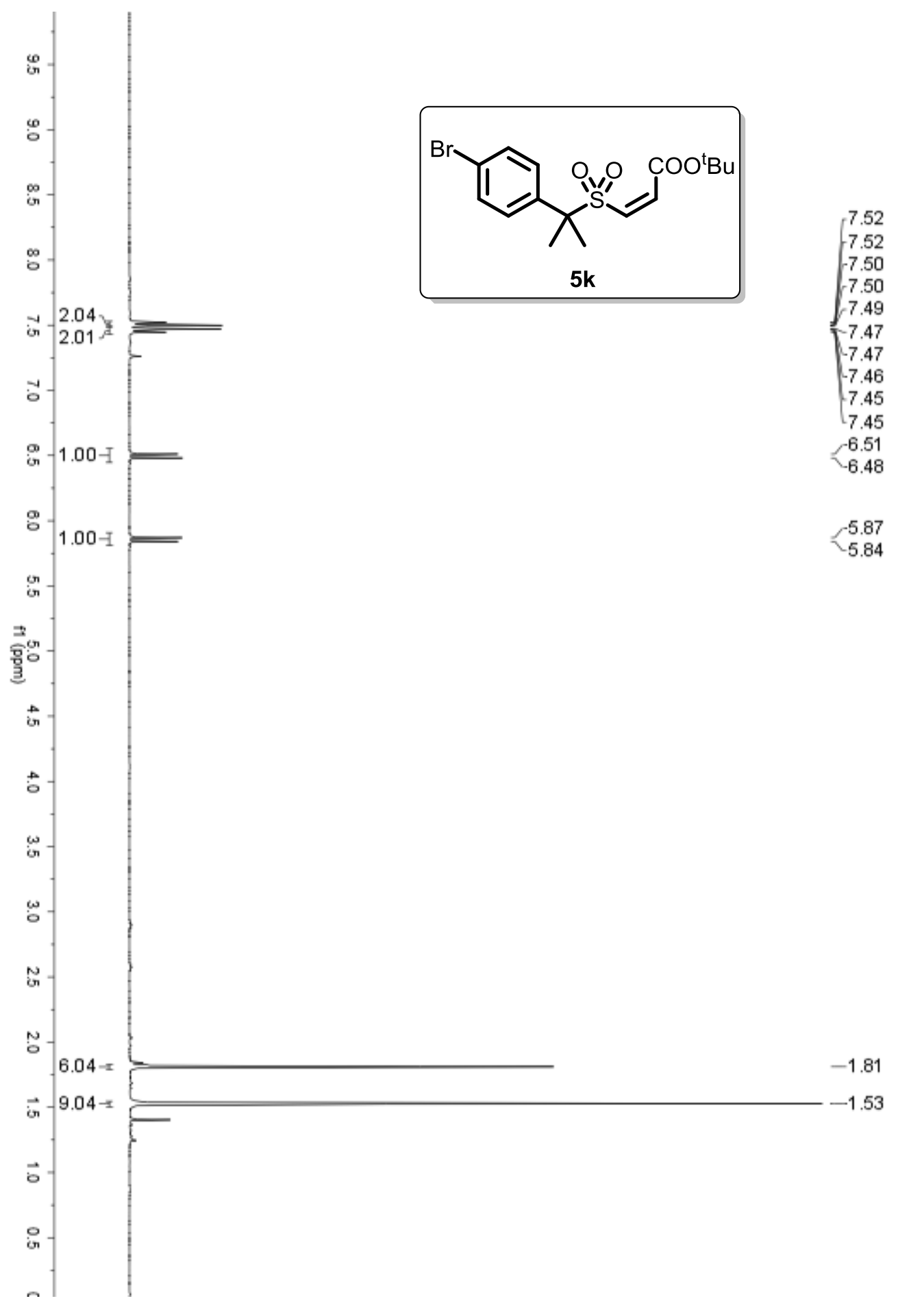


${ }^{13} \mathrm{C}$ NMR $\left(100 \mathrm{MHz}, \mathrm{CDCl}_{3}\right)$
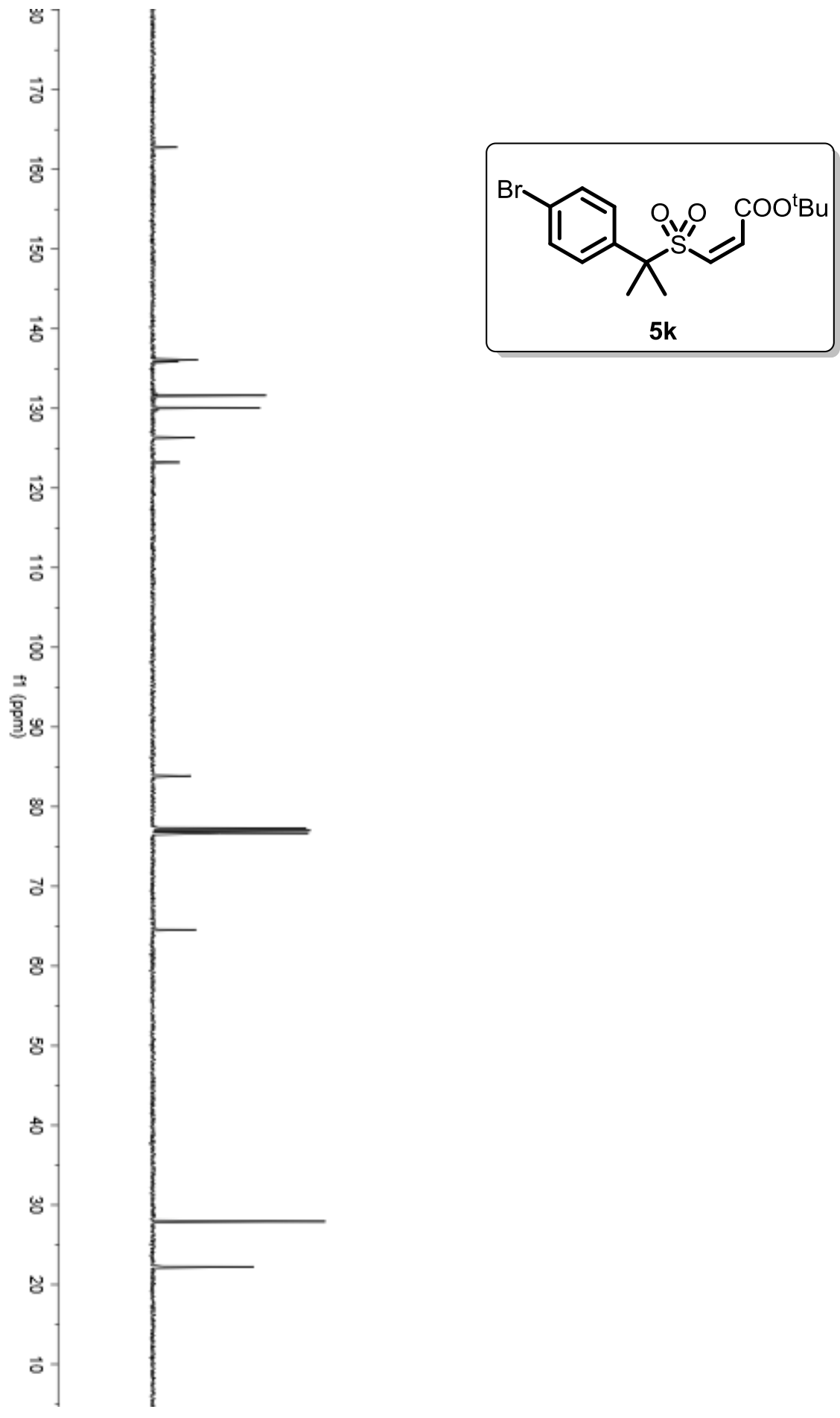

$-162.78$

< 136.10

$-135.89$

$-131.62$

$\checkmark 126.33$

$\checkmark 123.23$

$-83.87$

$-64.56$

$-27.93$

$-22.25$ 
${ }^{1} \mathbf{H}$ NMR $\left(400 \mathrm{MHz}, \mathrm{CDCl}_{3}\right)$

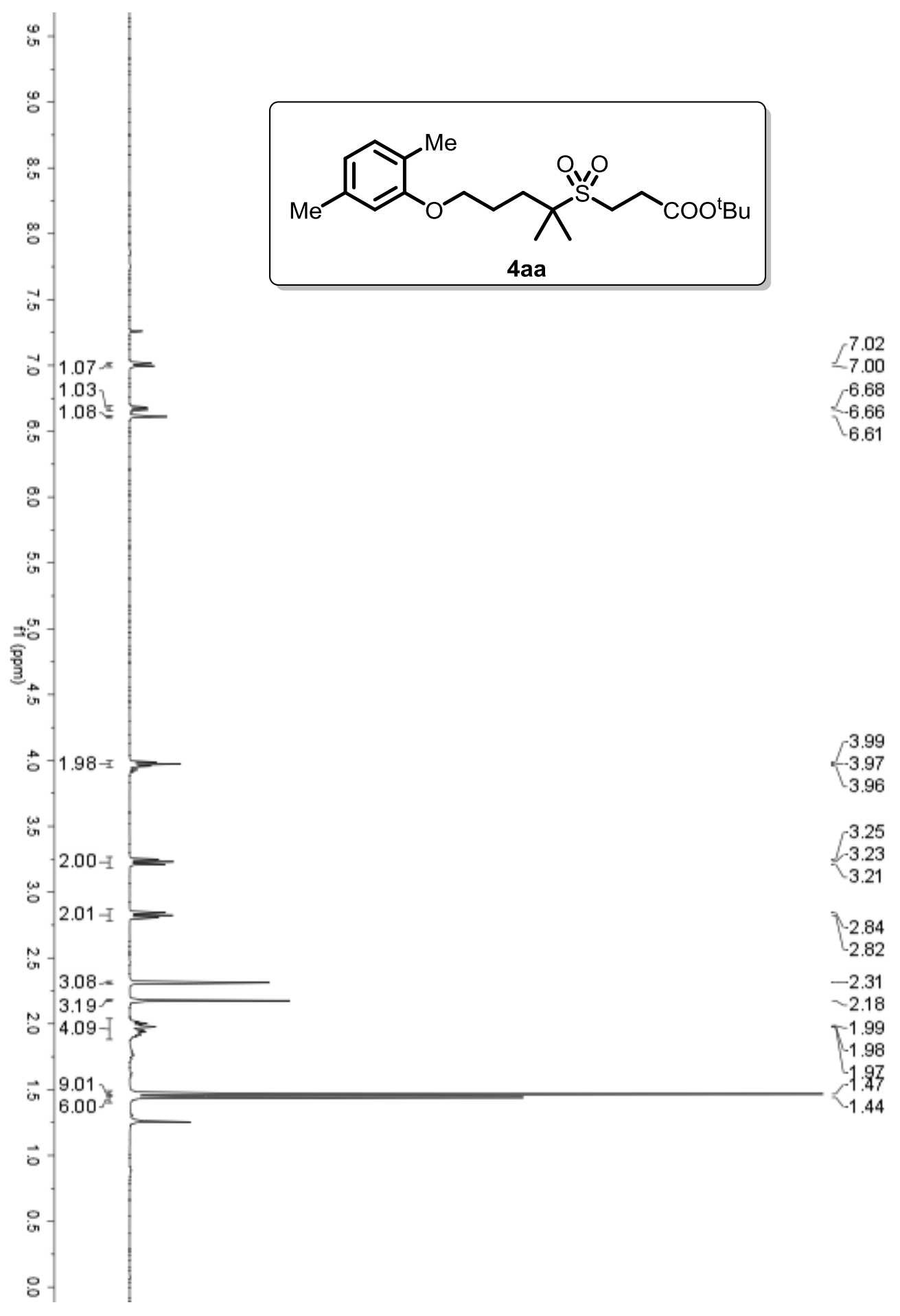


${ }^{13} \mathrm{C}$ NMR $\left(100 \mathrm{MHz}, \mathrm{CDCl}_{3}\right)$

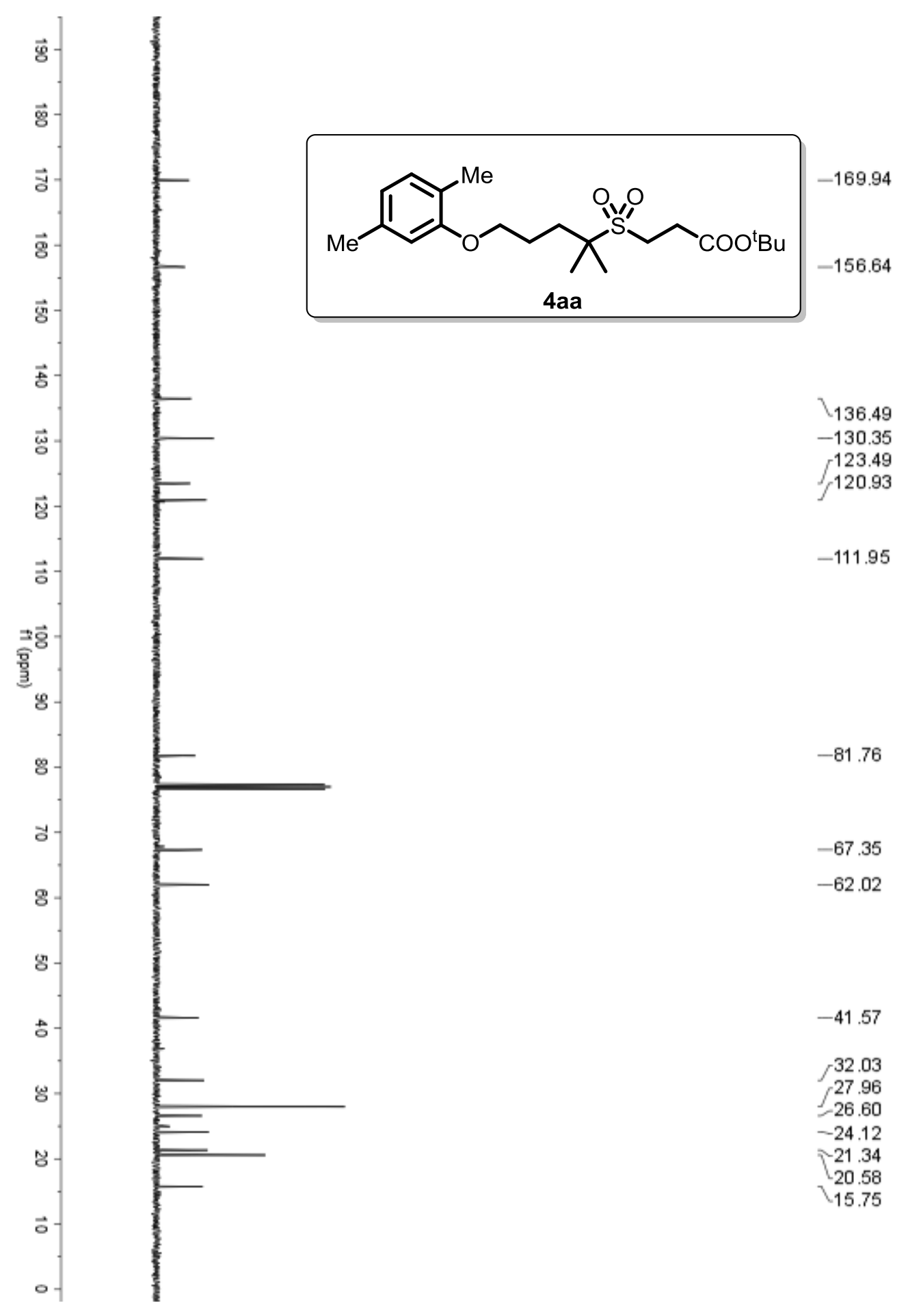


${ }^{1} \mathbf{H}$ NMR $\left(400 \mathrm{MHz}, \mathrm{CDCl}_{3}\right)$

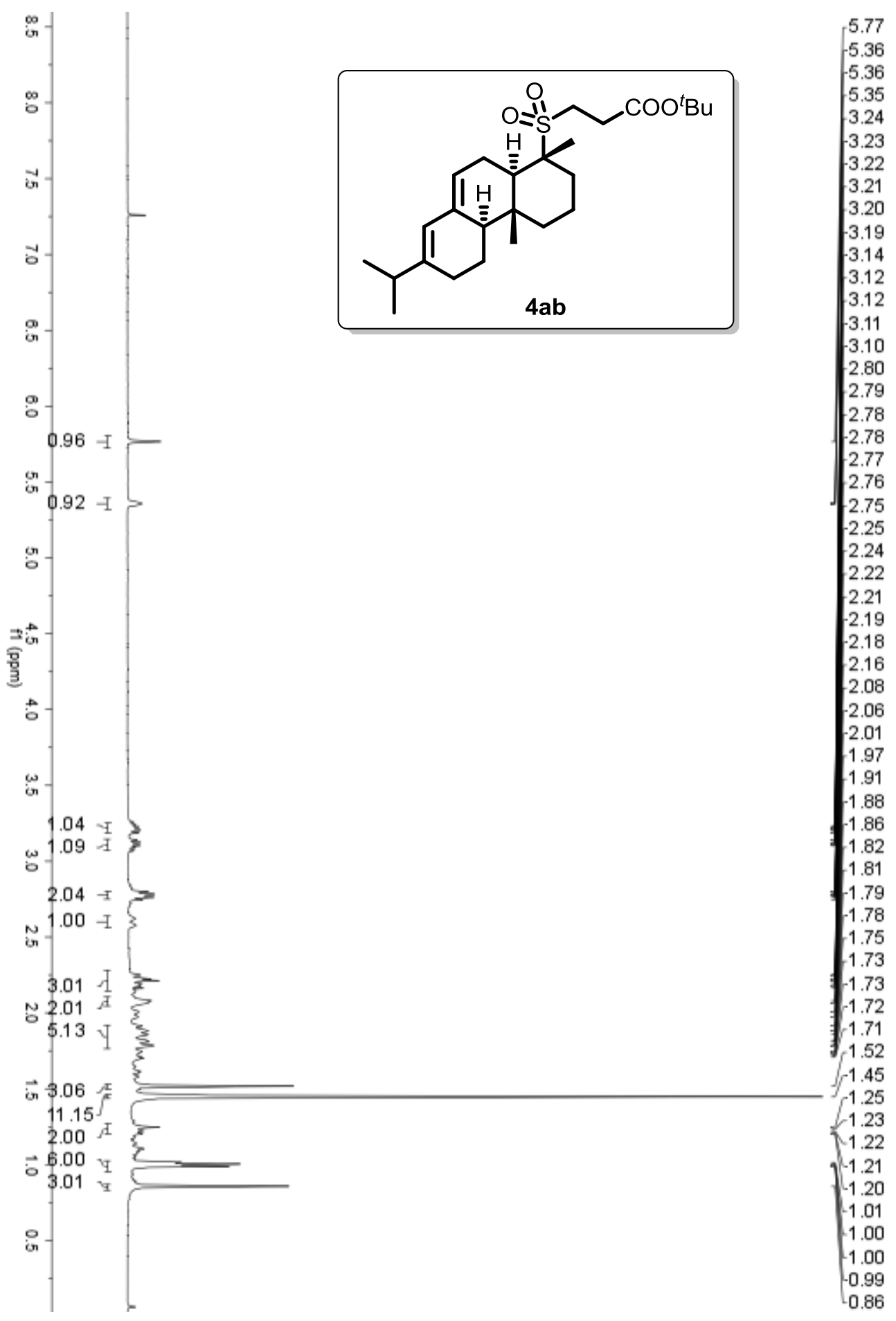


${ }^{13} \mathrm{C}$ NMR $\left(100 \mathrm{MHz}, \mathrm{CDCl}_{3}\right)$

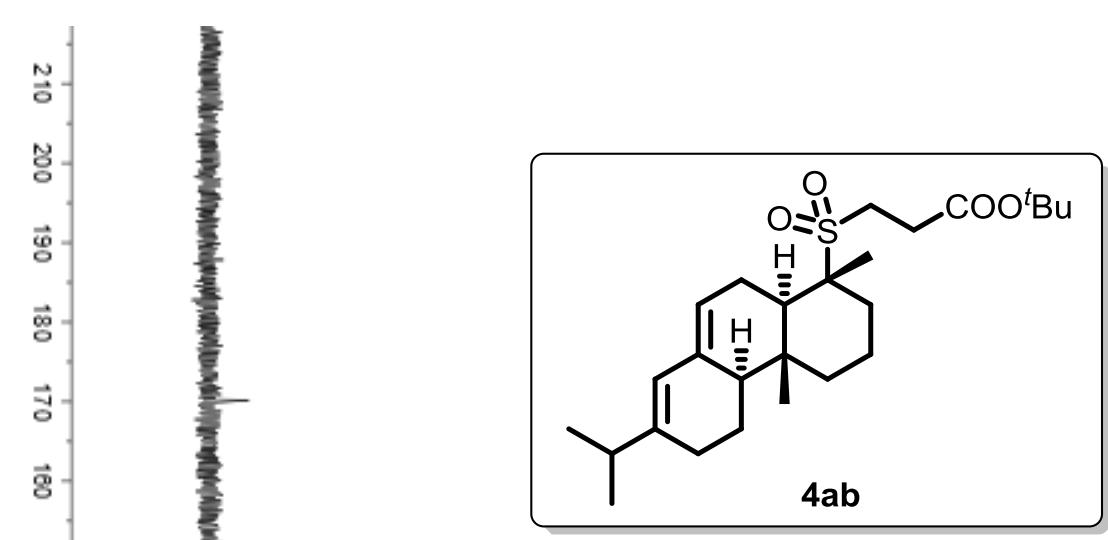


${ }^{1} \mathbf{H}$ NMR $\left(400 \mathrm{MHz}, \mathrm{CDCl}_{3}\right)$

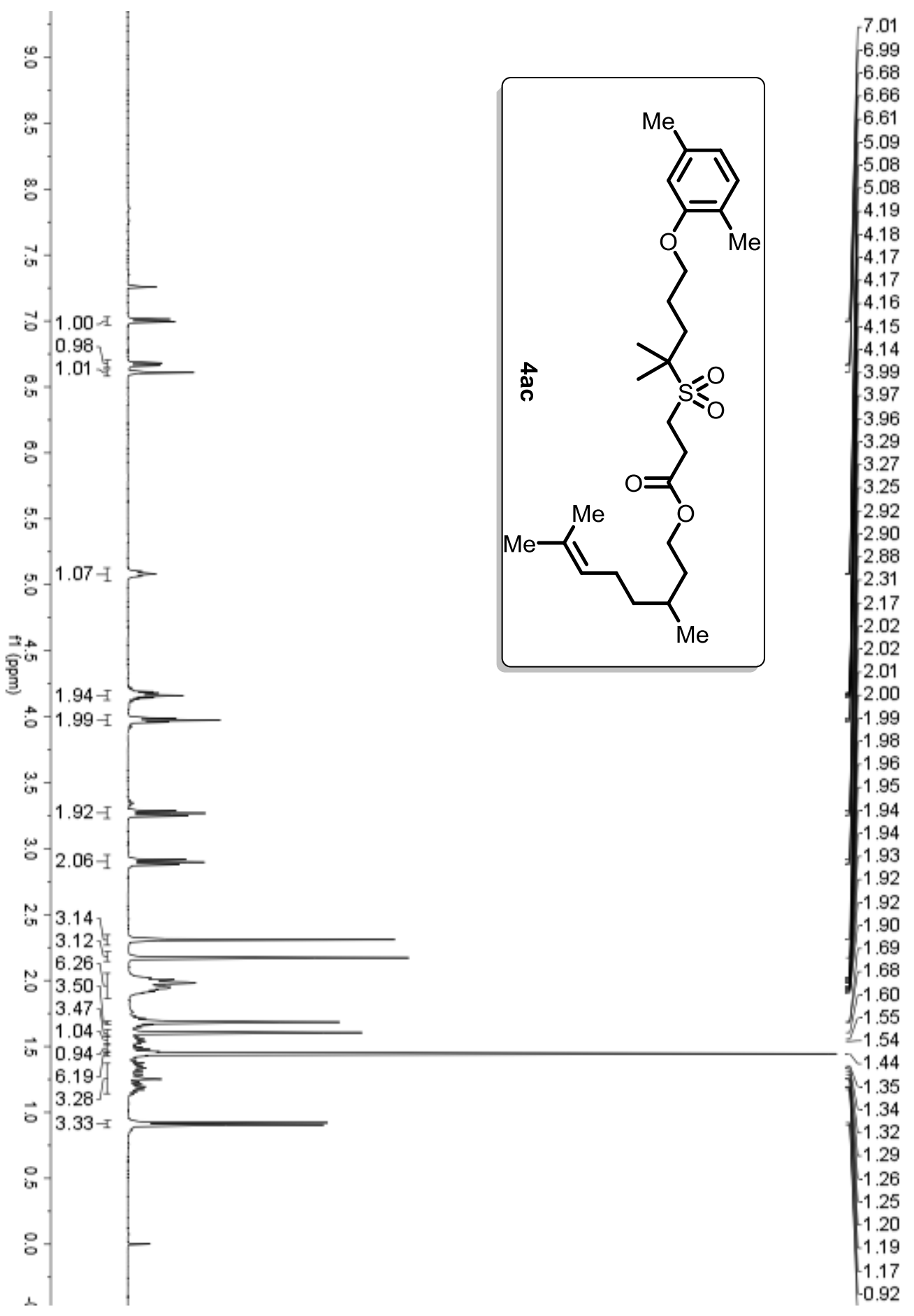


${ }^{13} \mathrm{C}$ NMR $\left(100 \mathrm{MHz}, \mathrm{CDCl}_{3}\right)$
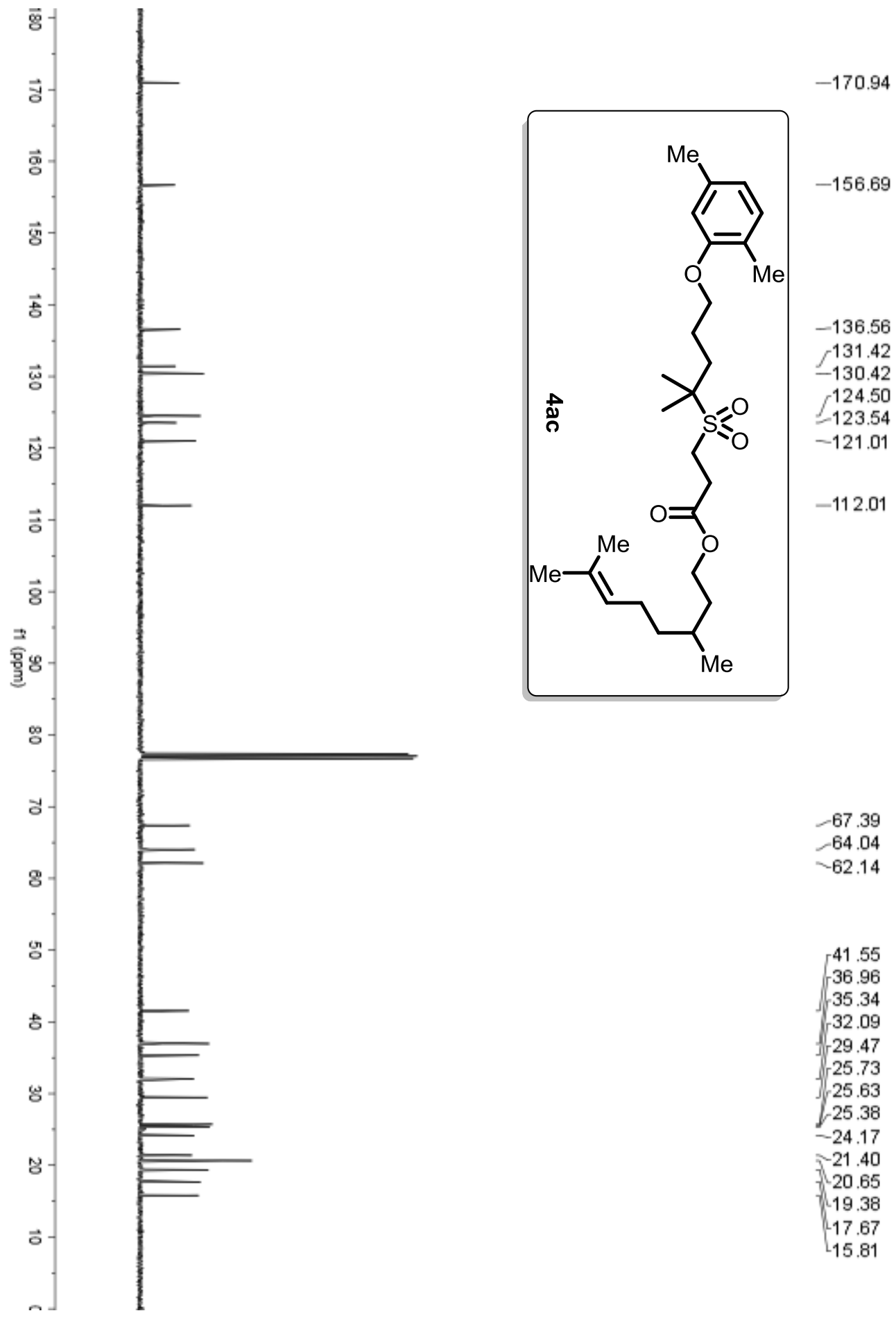
${ }^{1} \mathbf{H}$ NMR $\left(400 \mathrm{MHz}, \mathrm{CDCl}_{3}\right)$

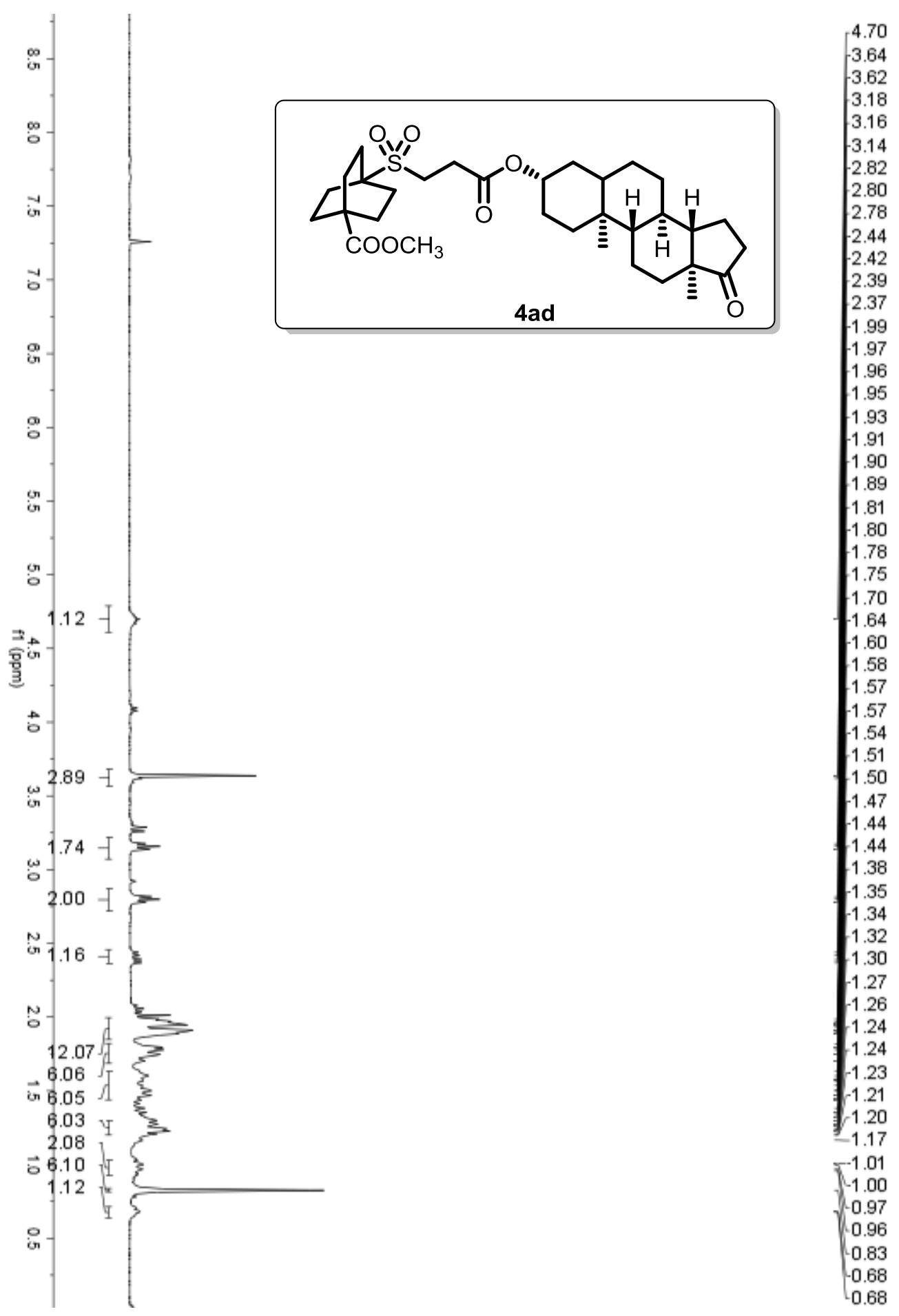


${ }^{13} \mathrm{C}$ NMR $\left(100 \mathrm{MHz}, \mathrm{CDCl}_{3}\right)$

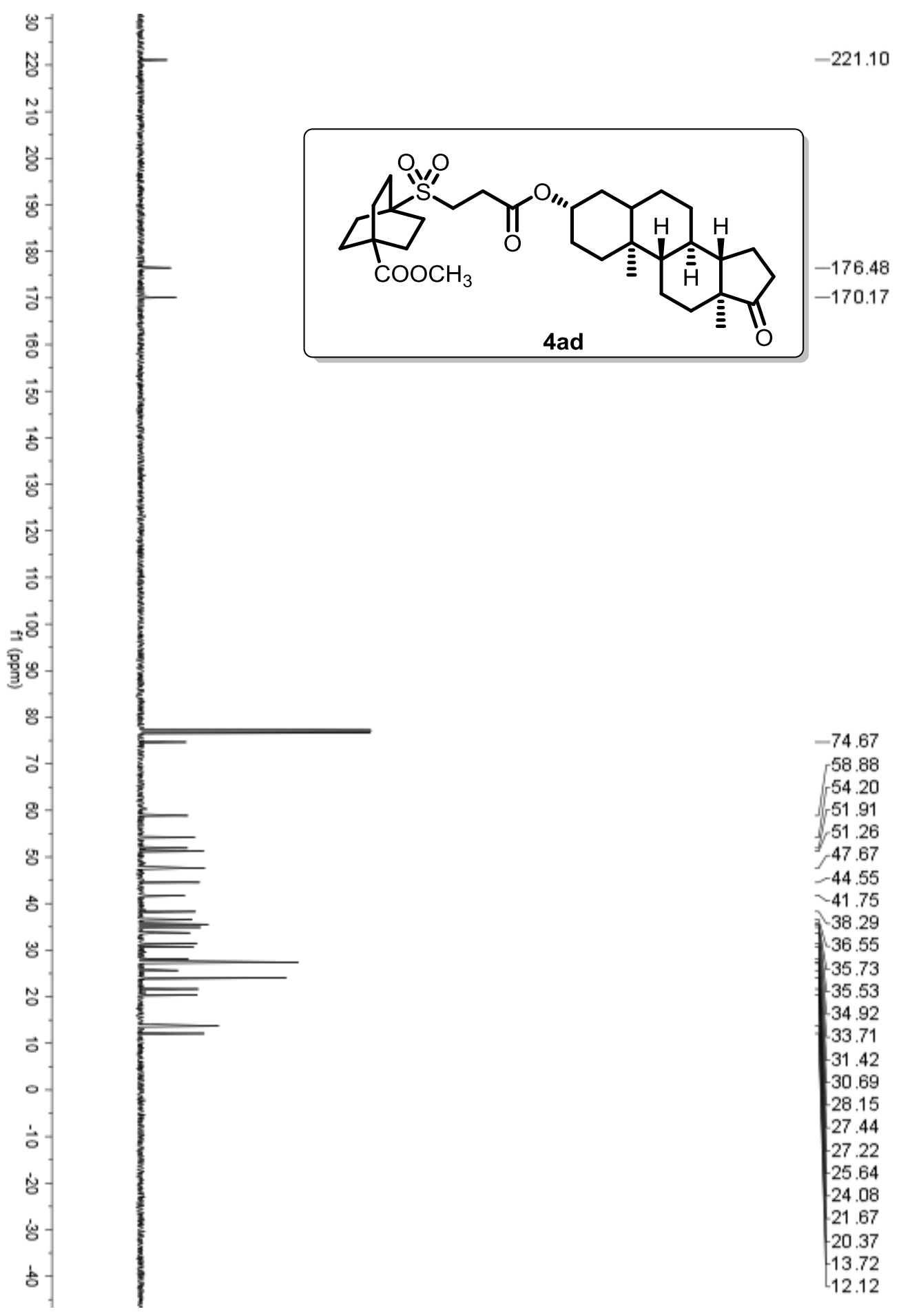


${ }^{1} \mathbf{H}$ NMR $\left(400 \mathrm{MHz}, \mathrm{CDCl}_{3}\right)$

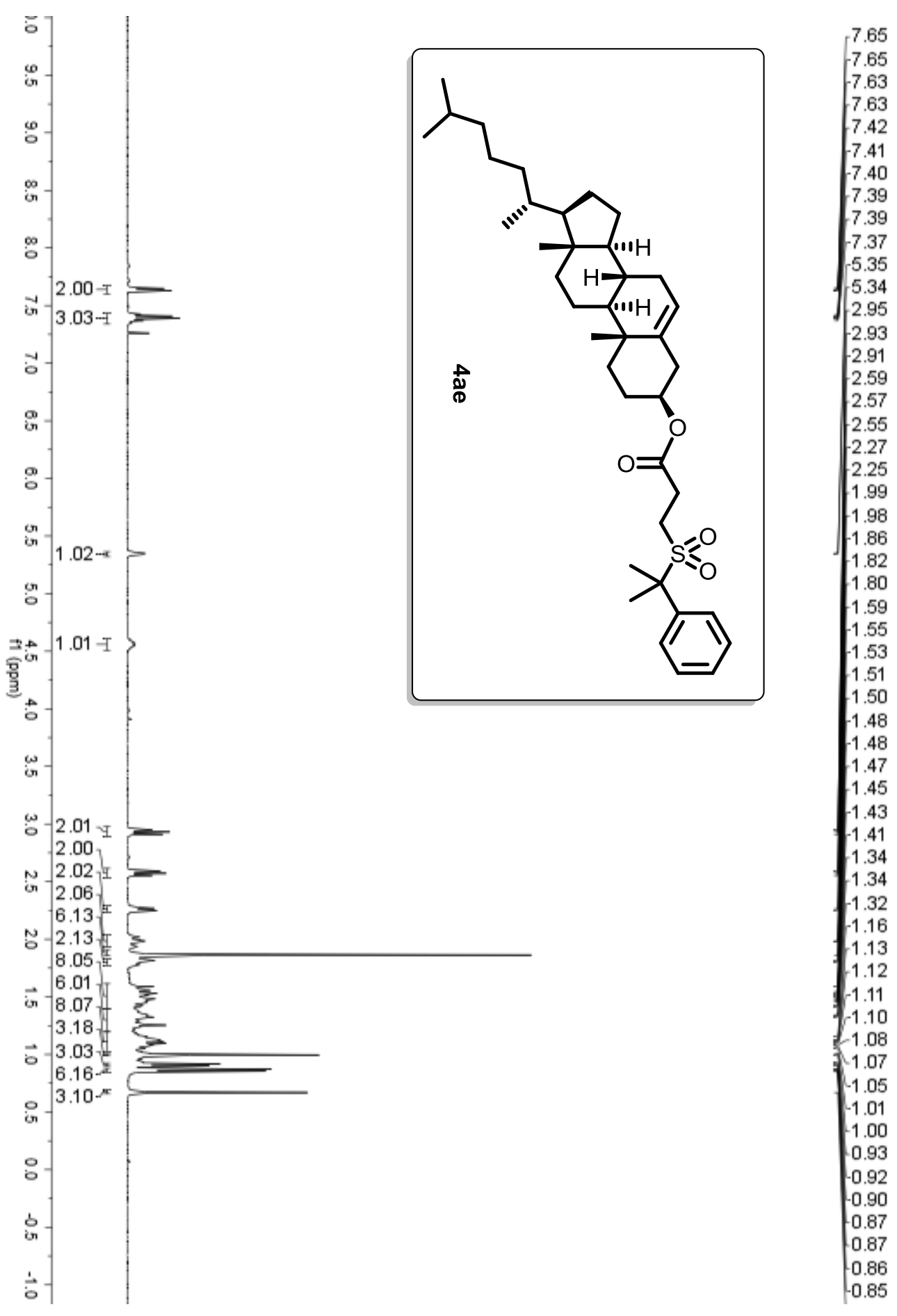


${ }^{13} \mathrm{C}$ NMR $\left(100 \mathrm{MHz}, \mathrm{CDCl}_{3}\right)$

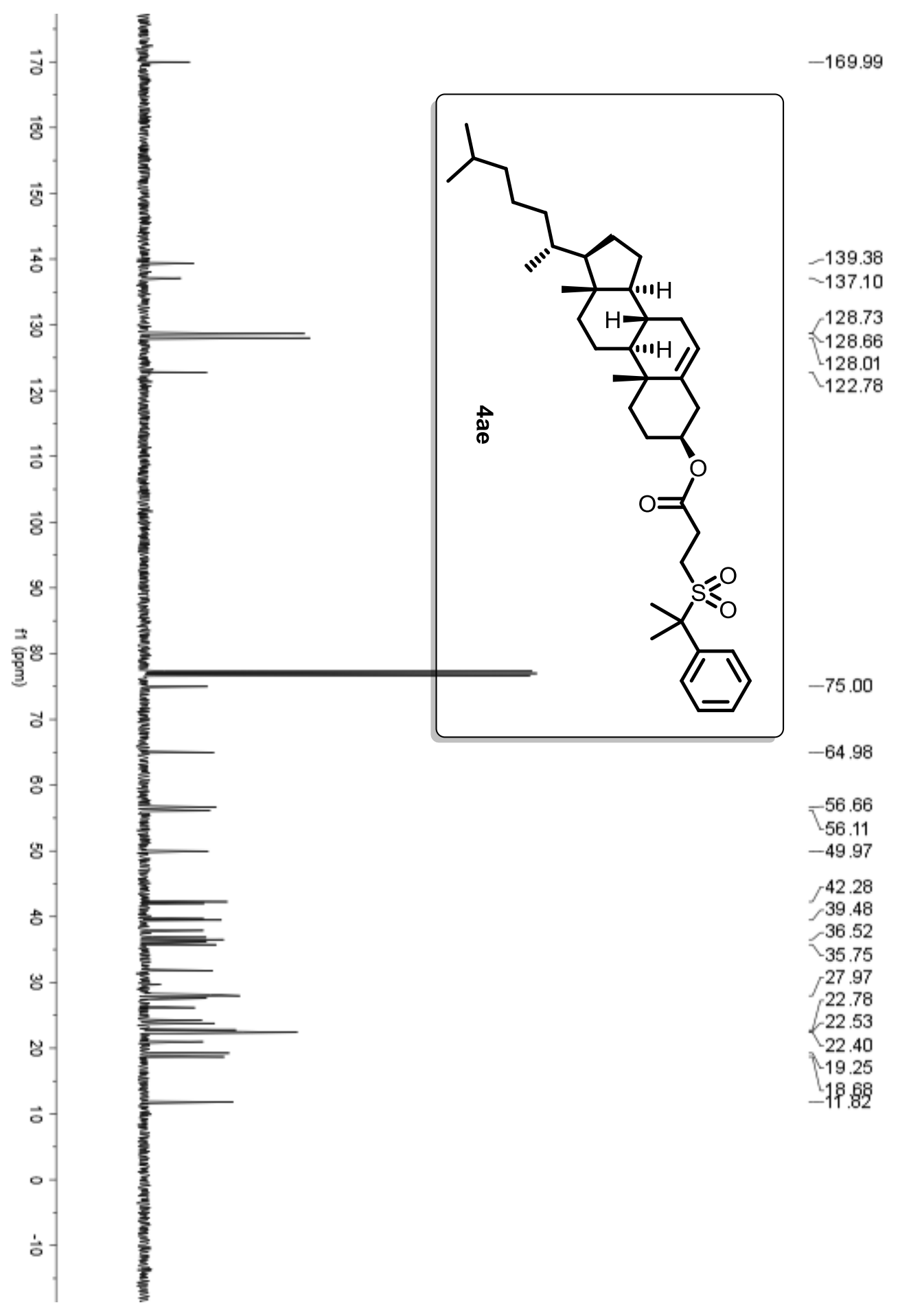


${ }^{1} \mathbf{H}$ NMR $\left(400 \mathrm{MHz}, \mathrm{CDCl}_{3}\right)$

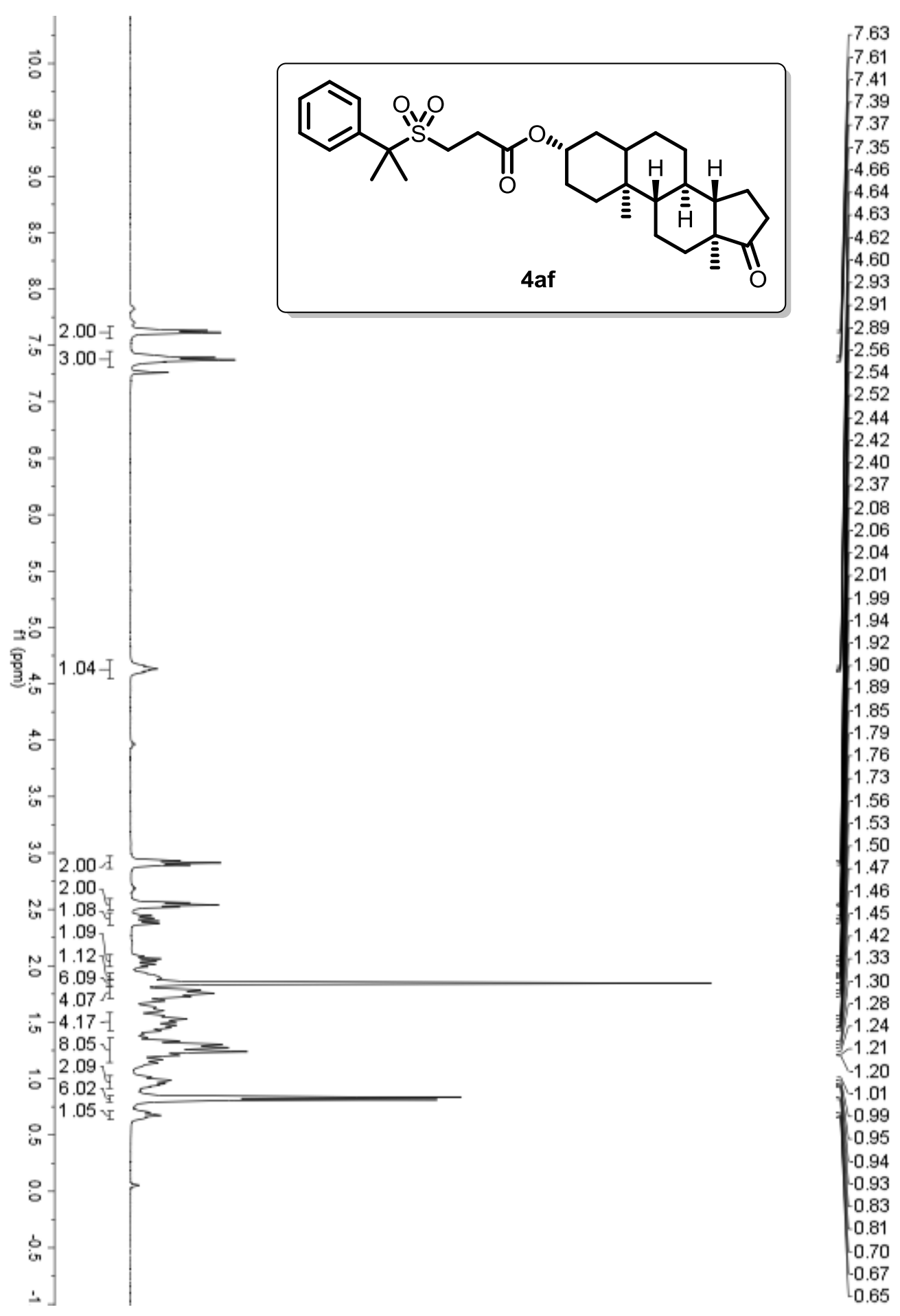


${ }^{13} \mathrm{C}$ NMR $\left(100 \mathrm{MHz}, \mathrm{CDCl}_{3}\right)$

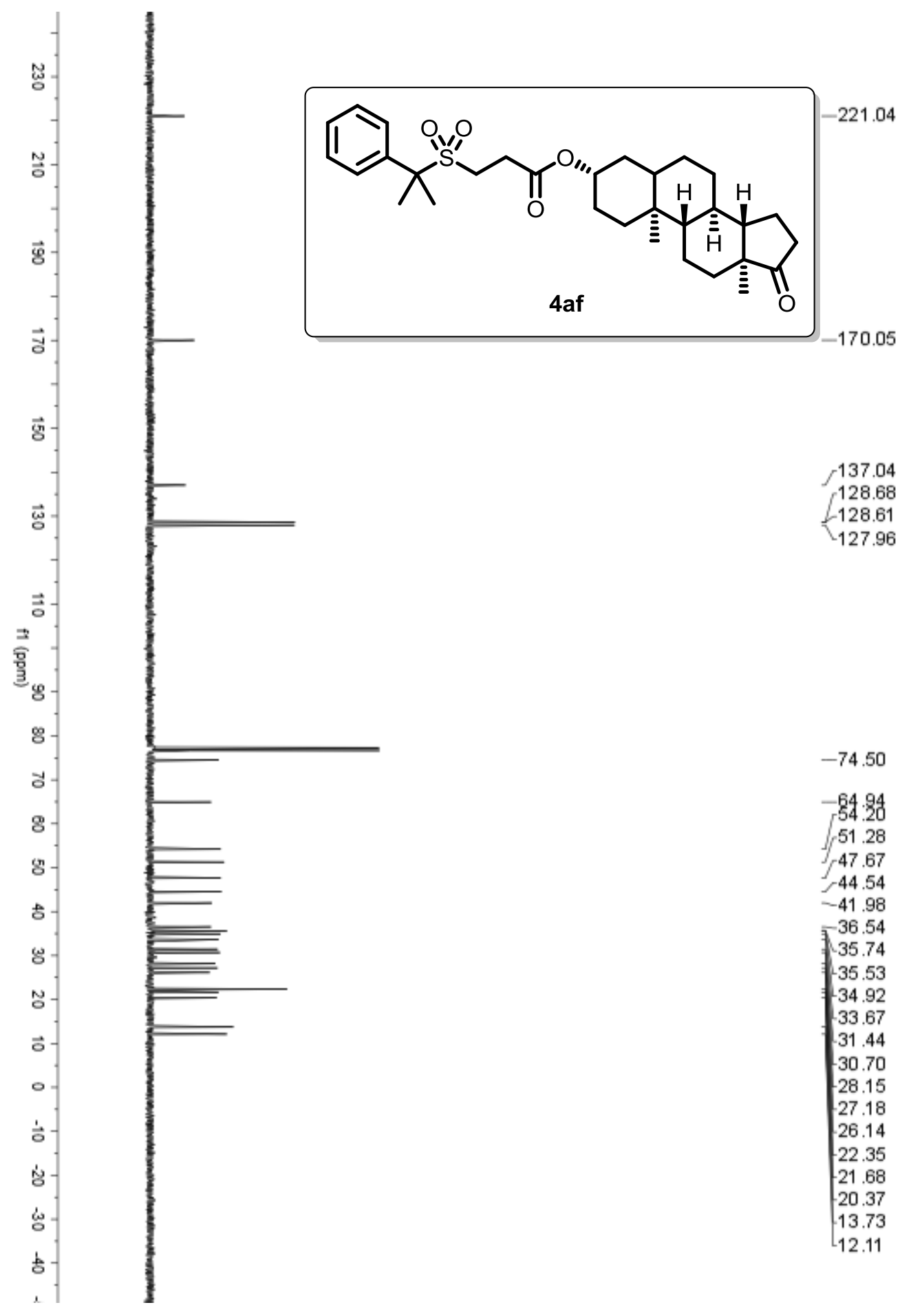


${ }^{1} \mathbf{H}$ NMR $\left(400 \mathrm{MHz}, \mathrm{CDCl}_{3}\right)$

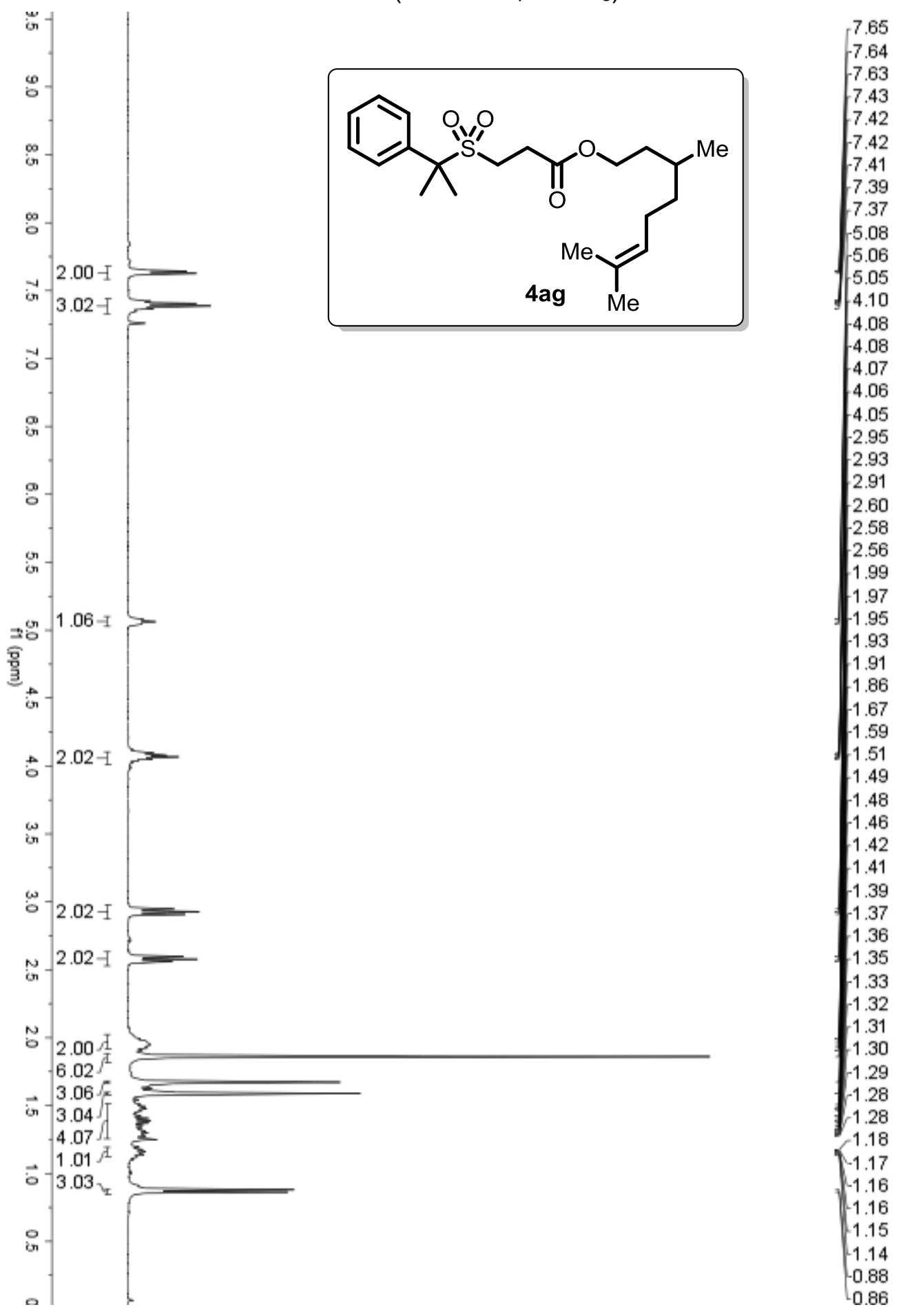


${ }^{13} \mathrm{C}$ NMR $\left(100 \mathrm{MHz}, \mathrm{CDCl}_{3}\right)$

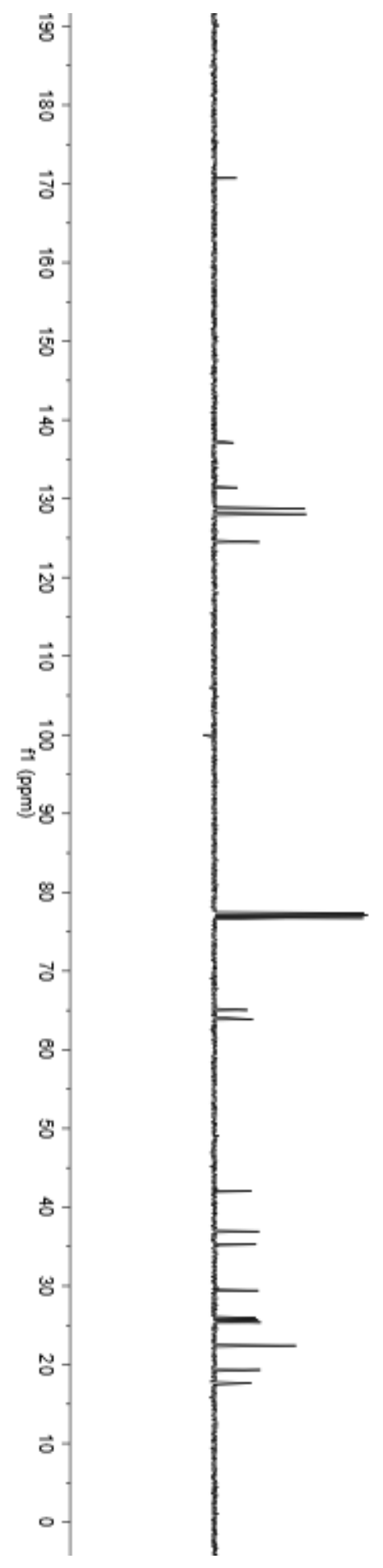

$-170.71$

137.11

131.41

128.80

$-128.71$

$-128.05$

$\searrow_{124.48}$

$-65.05$

$\checkmark 63.86$

42.03

36.93 r 35.27

29.42

25.94

25.72

$<25.36$

$-22.43$

$-19.35$

17.66 
${ }^{1} \mathbf{H}$ NMR $\left(400 \mathrm{MHz}, \mathrm{CDCl}_{3}\right)$
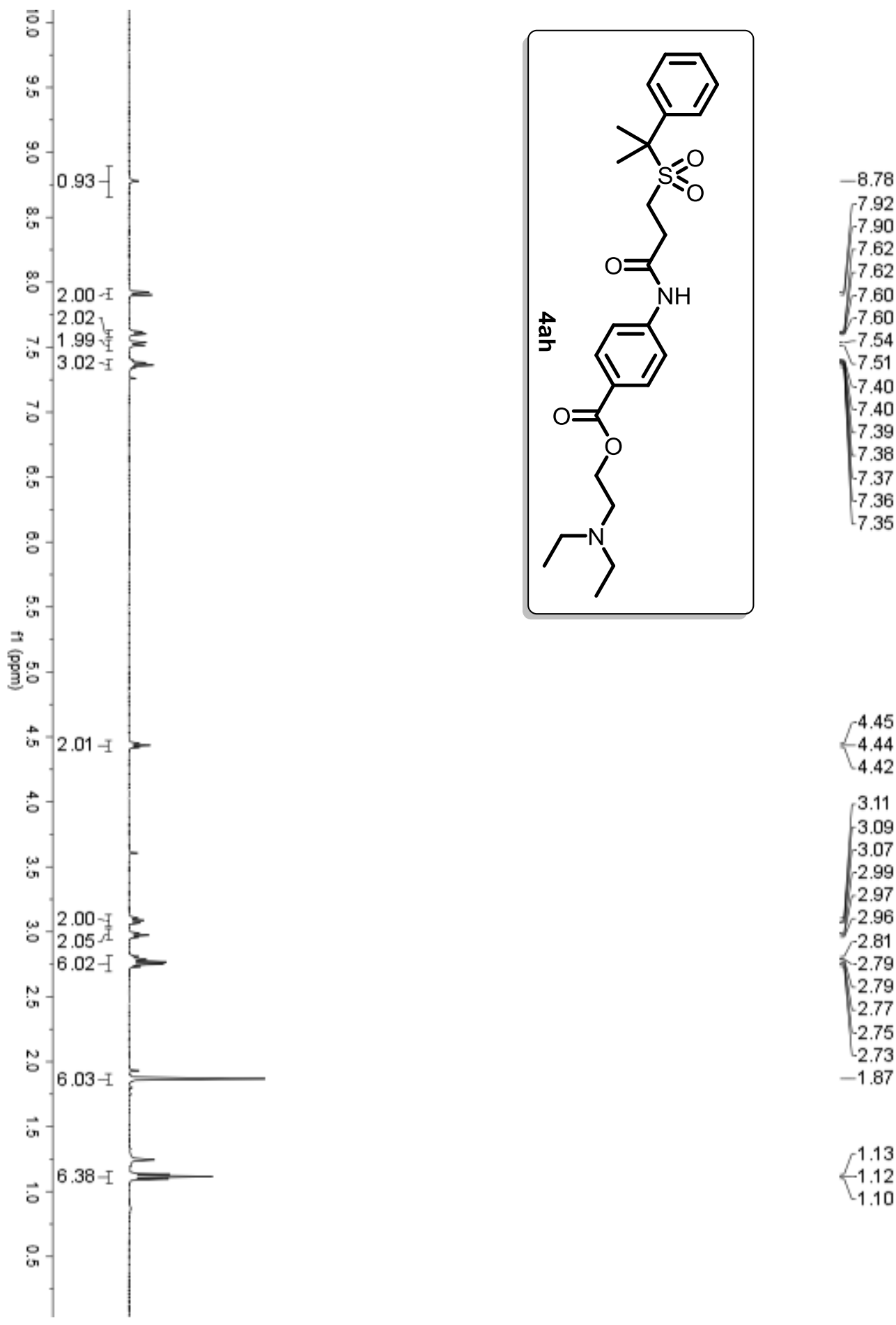

3.11

$-3.09$

-3.07
-2.99

2.97

2.96
-2.96

2.81

2.79

( 2.79

2.77

$-2.75$

$-2.73$

$-1.87$

$\leftarrow_{-1.13}^{1.12}$

$\bigcup_{1.10}$ 
${ }^{13} \mathrm{C}$ NMR $\left(100 \mathrm{MHz}, \mathrm{CDCl}_{3}\right)$
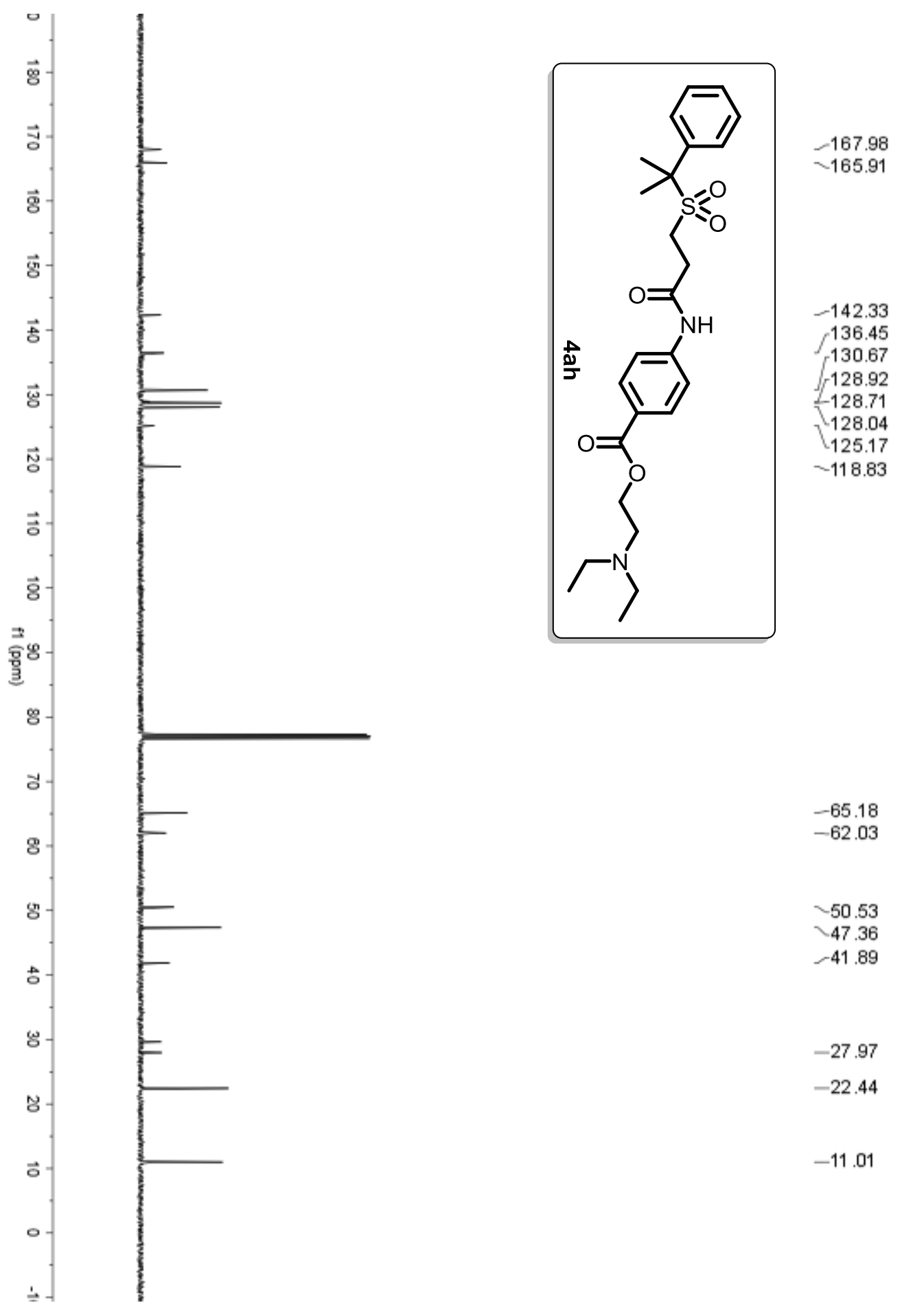

$-65.18$

$-62.03$

$-50.53$

$\checkmark 4.36$

$-41.89$

$-27.97$

$-22.44$

$-11.01$ 
${ }^{1} \mathbf{H}$ NMR $\left(400 \mathrm{MHz}, \mathrm{CDCl}_{3}\right)$

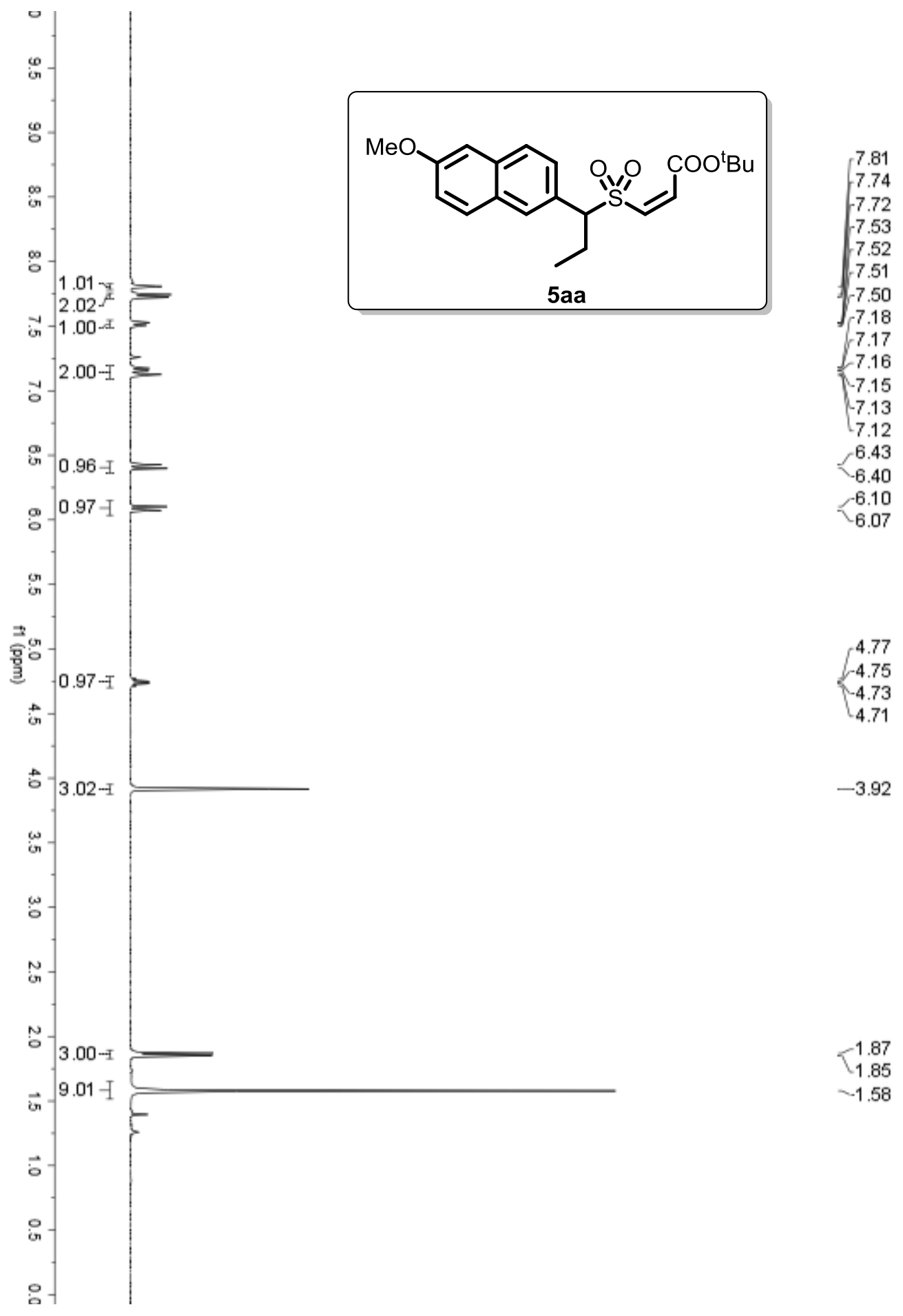


${ }^{13} \mathrm{C}$ NMR $\left(100 \mathrm{MHz}, \mathrm{CDCl}_{3}\right)$

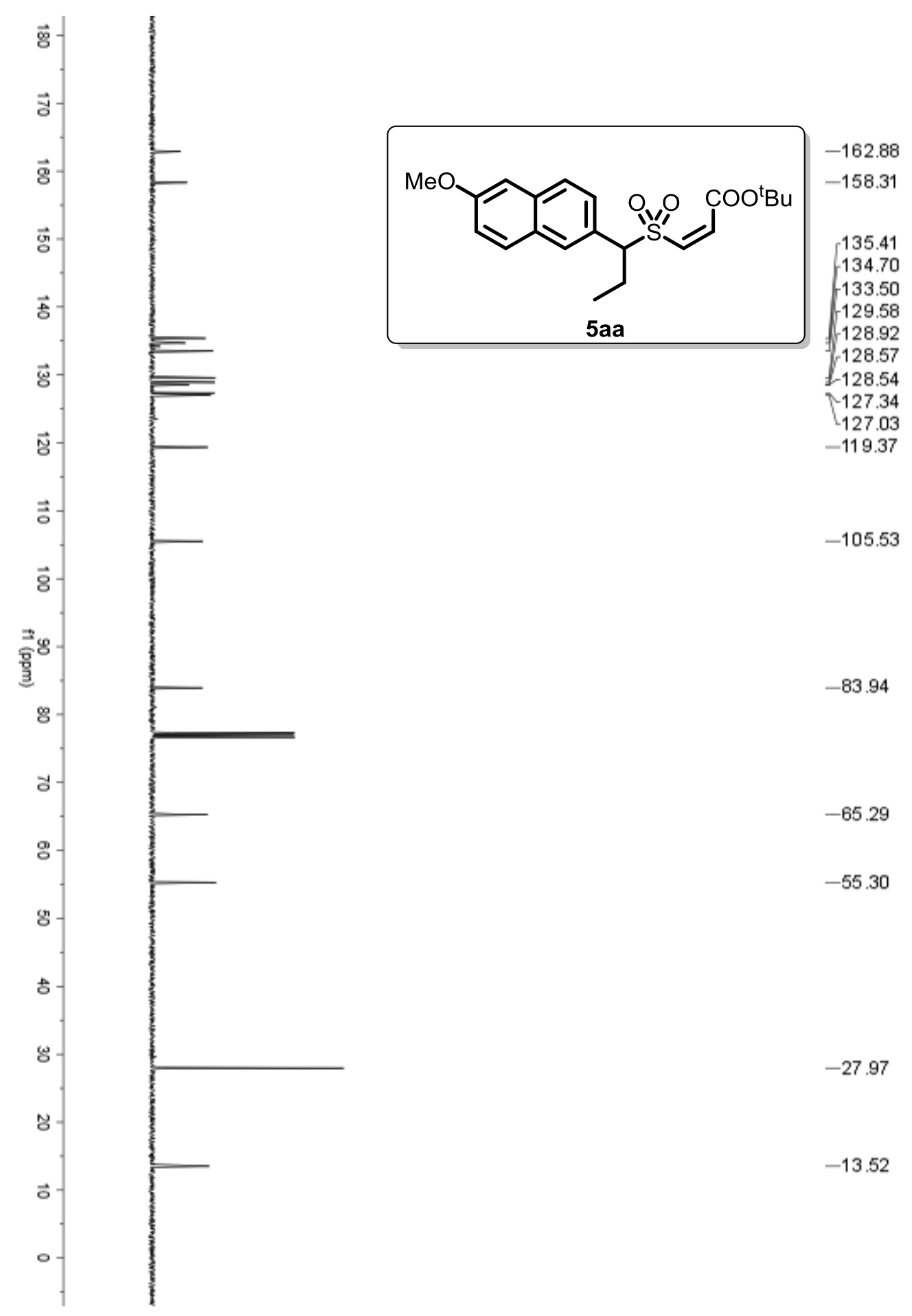


${ }^{1} \mathbf{H}$ NMR $\left(400 \mathrm{MHz}, \mathrm{CDCl}_{3}\right)$

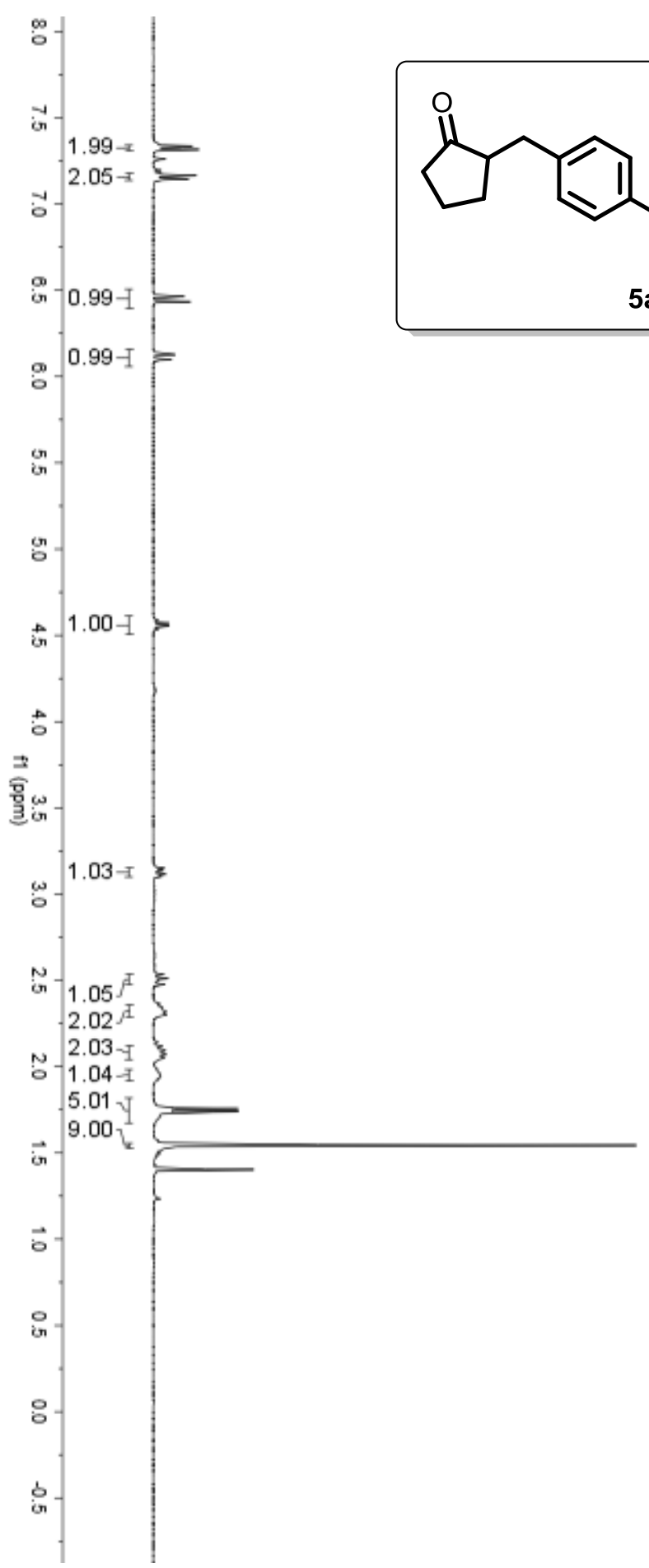

4.59
-4.57
-4.55
4.54 
${ }^{13} \mathrm{C}$ NMR $\left(100 \mathrm{MHz}, \mathrm{CDCl}_{3}\right)$
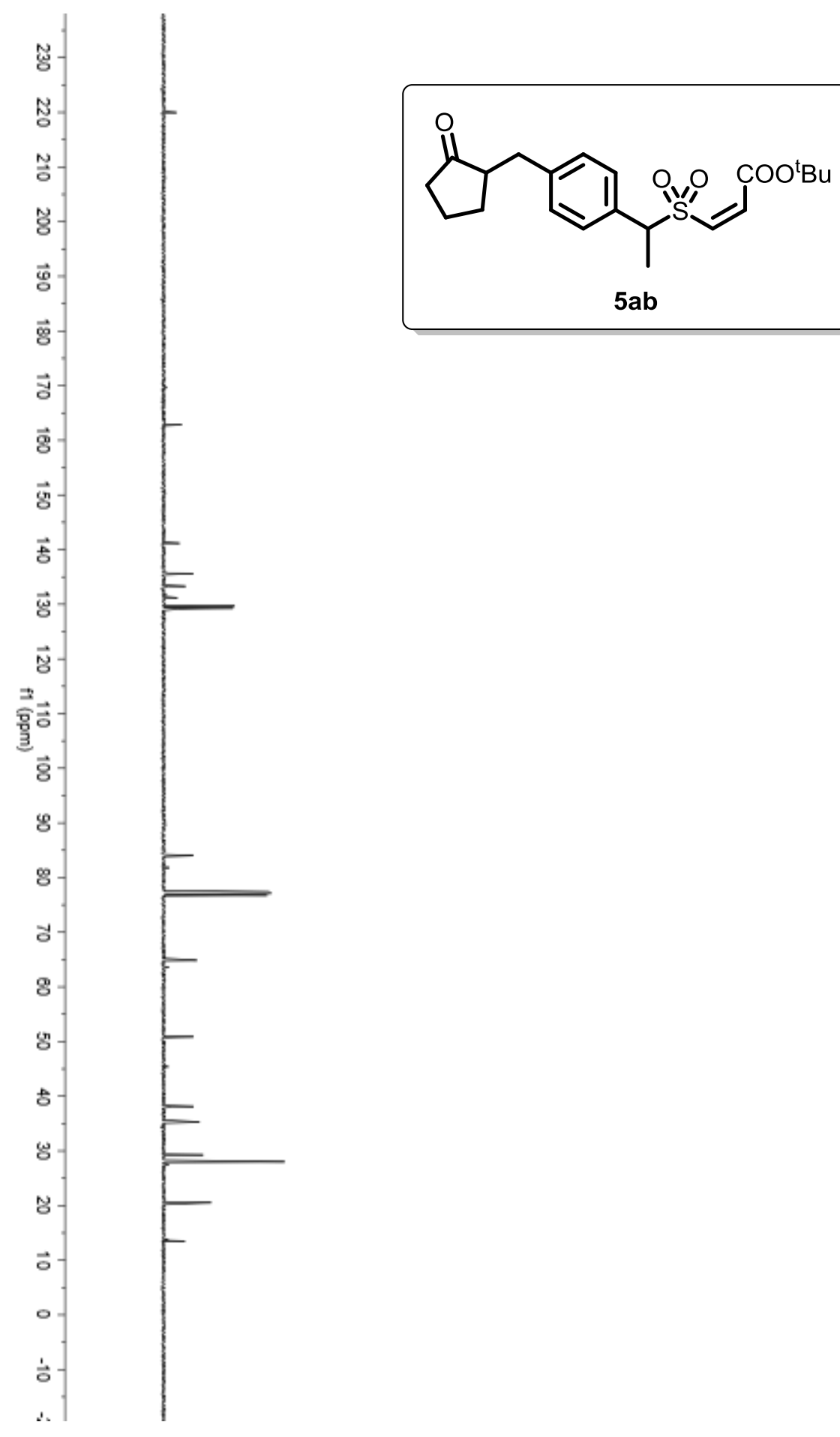

$-219.96$

$-162.87$

141.16

$\int_{r} 135.64$

133.30

131.17

129.66

$-129.32$

$-84.02$

$-64.85$

$-50.87$

$\checkmark 38.12$

$-35.28$

$-29.27$

28.01

$\checkmark 20.53$

$-13.50$ 
${ }^{1} \mathbf{H}$ NMR $\left(400 \mathrm{MHz}, \mathrm{CDCl}_{3}\right)$

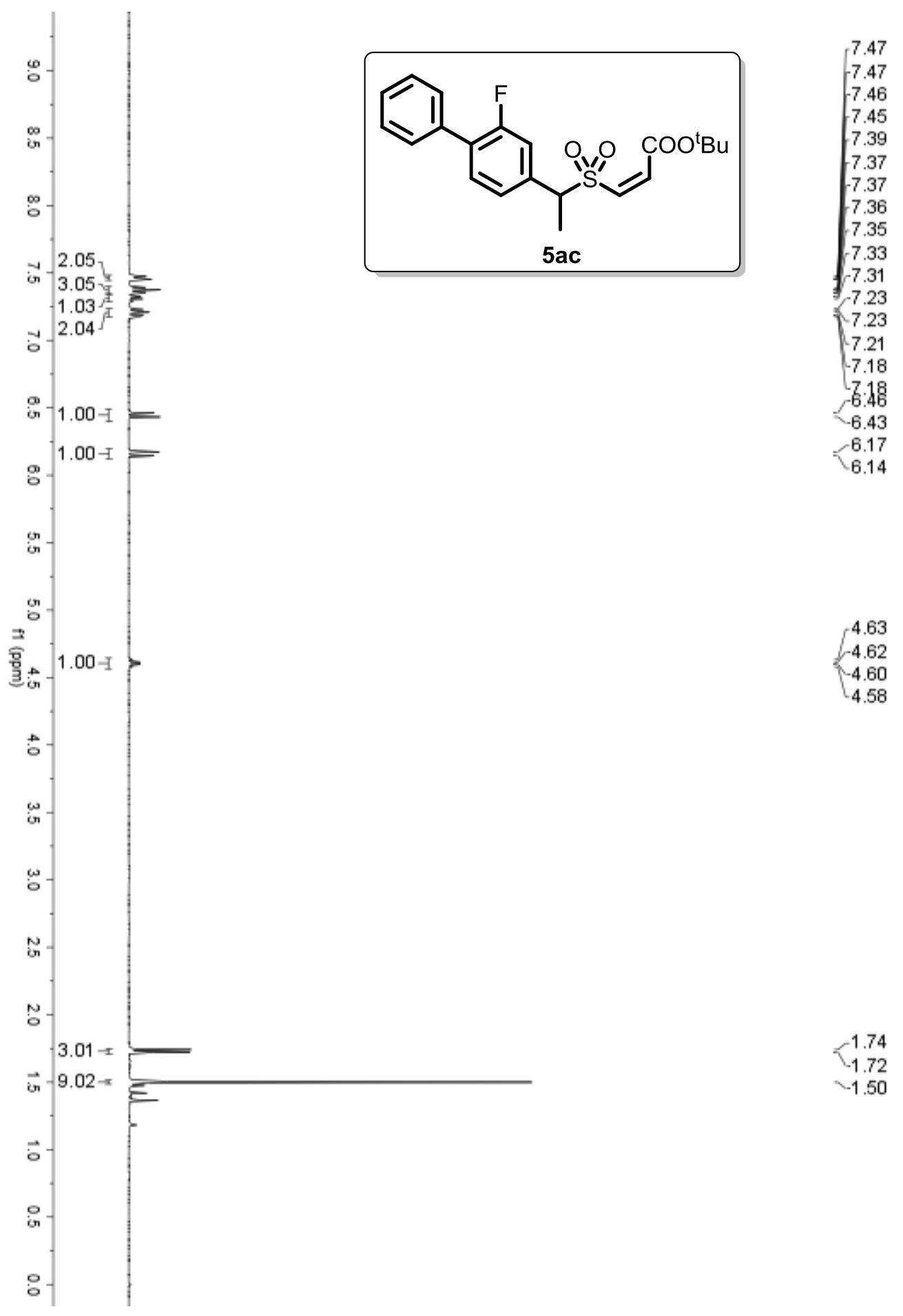


${ }^{13}$ C NMR $\left(100 \mathrm{MHz}, \mathrm{CDCl}_{3}\right)$

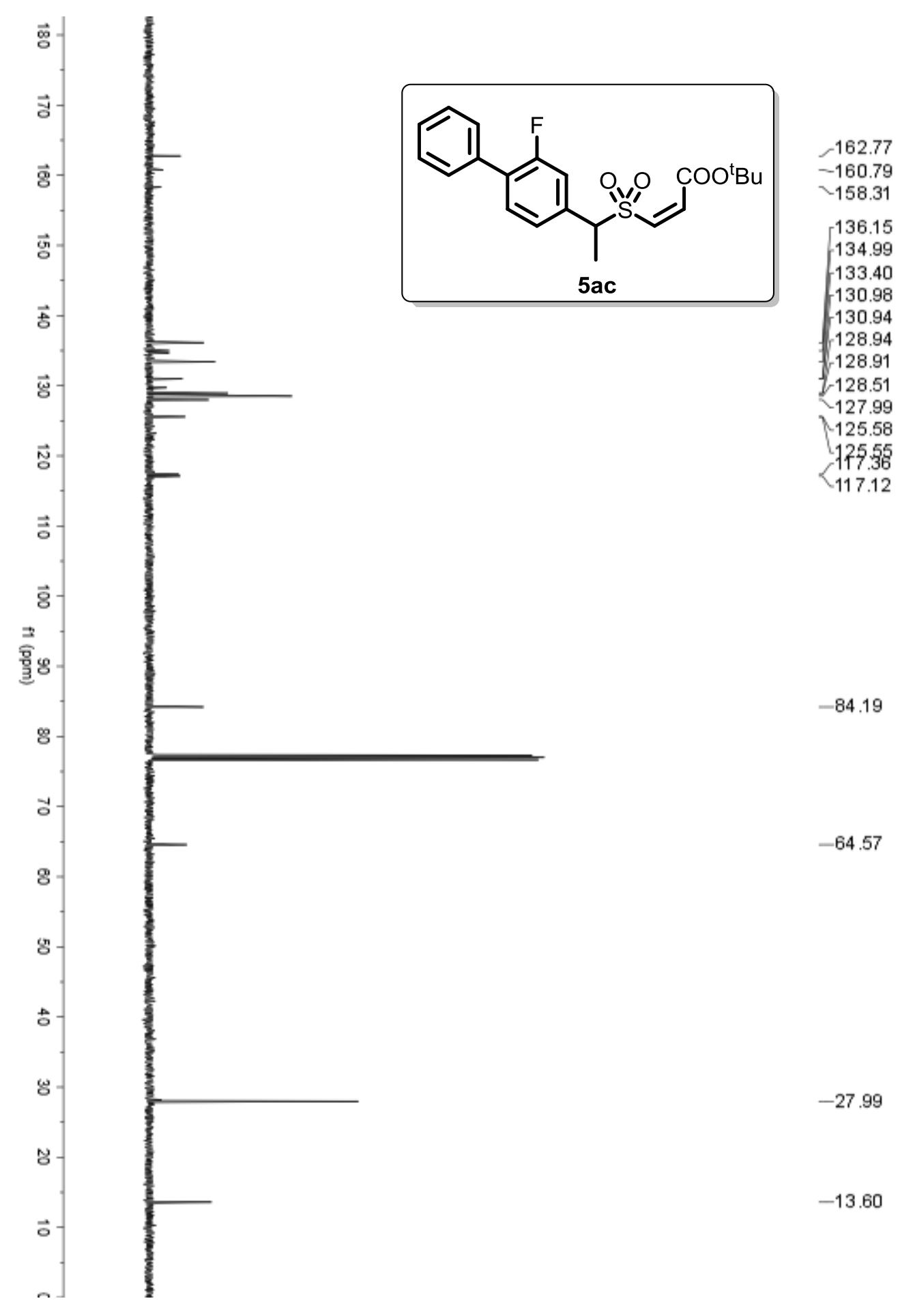


${ }^{19} \mathrm{~F}$ NMR $\left(376 \mathrm{MHz}, \mathrm{CDCl}_{3}\right)$

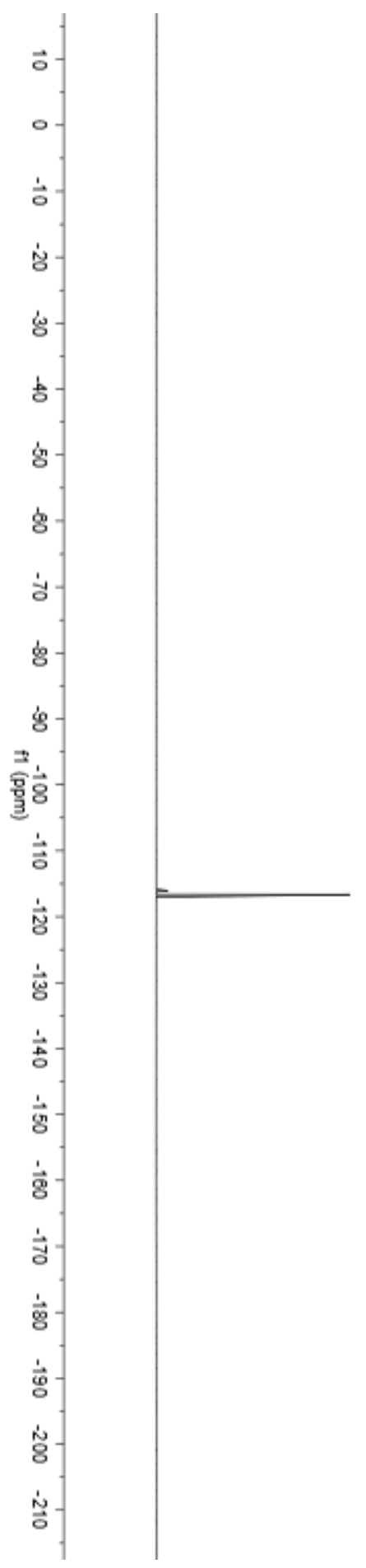


${ }^{1} \mathbf{H}$ NMR $\left(400 \mathrm{MHz}, \mathrm{CDCl}_{3}\right)$
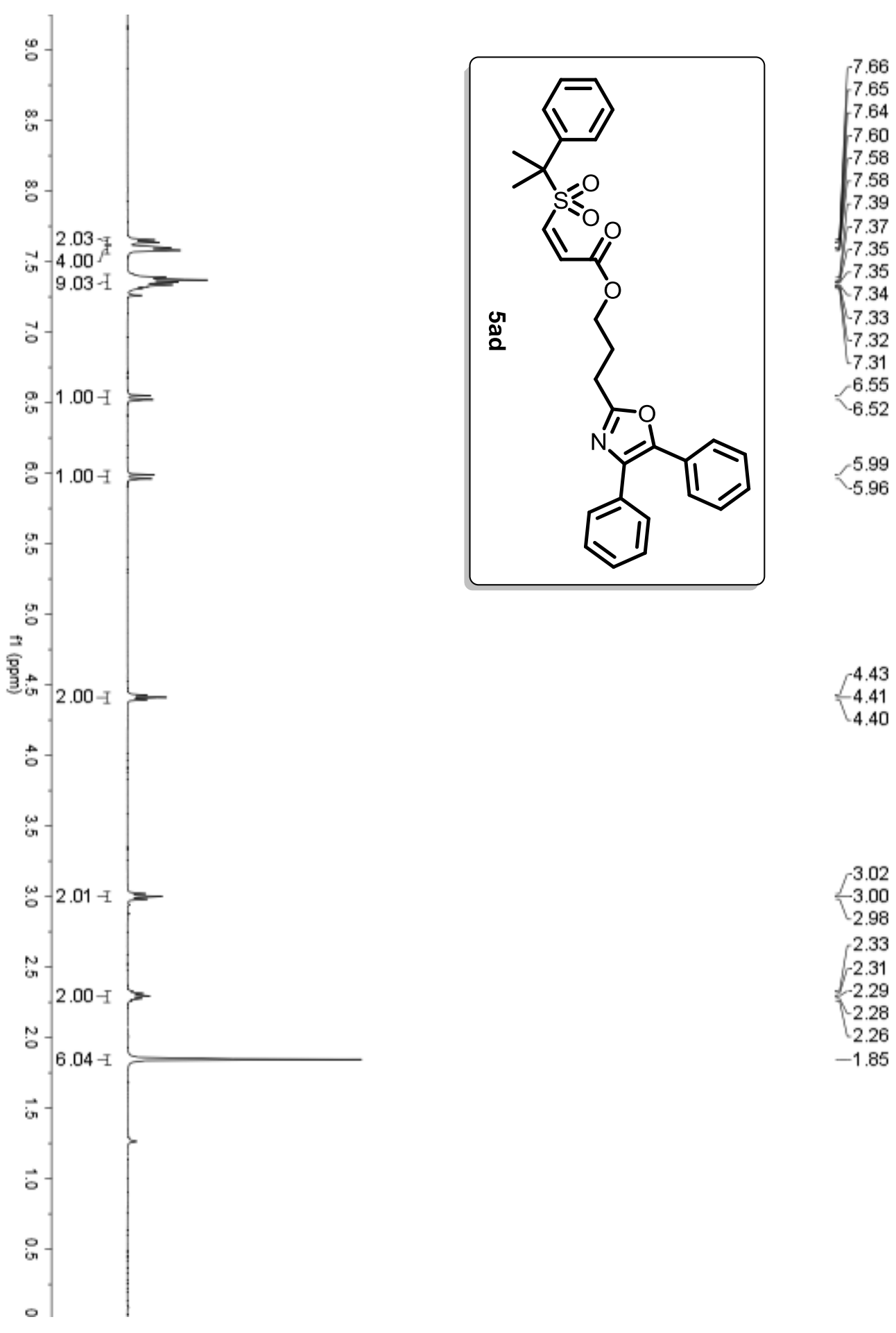
${ }^{13} \mathrm{C}$ NMR $\left(100 \mathrm{MHz}, \mathrm{CDCl}_{3}\right)$

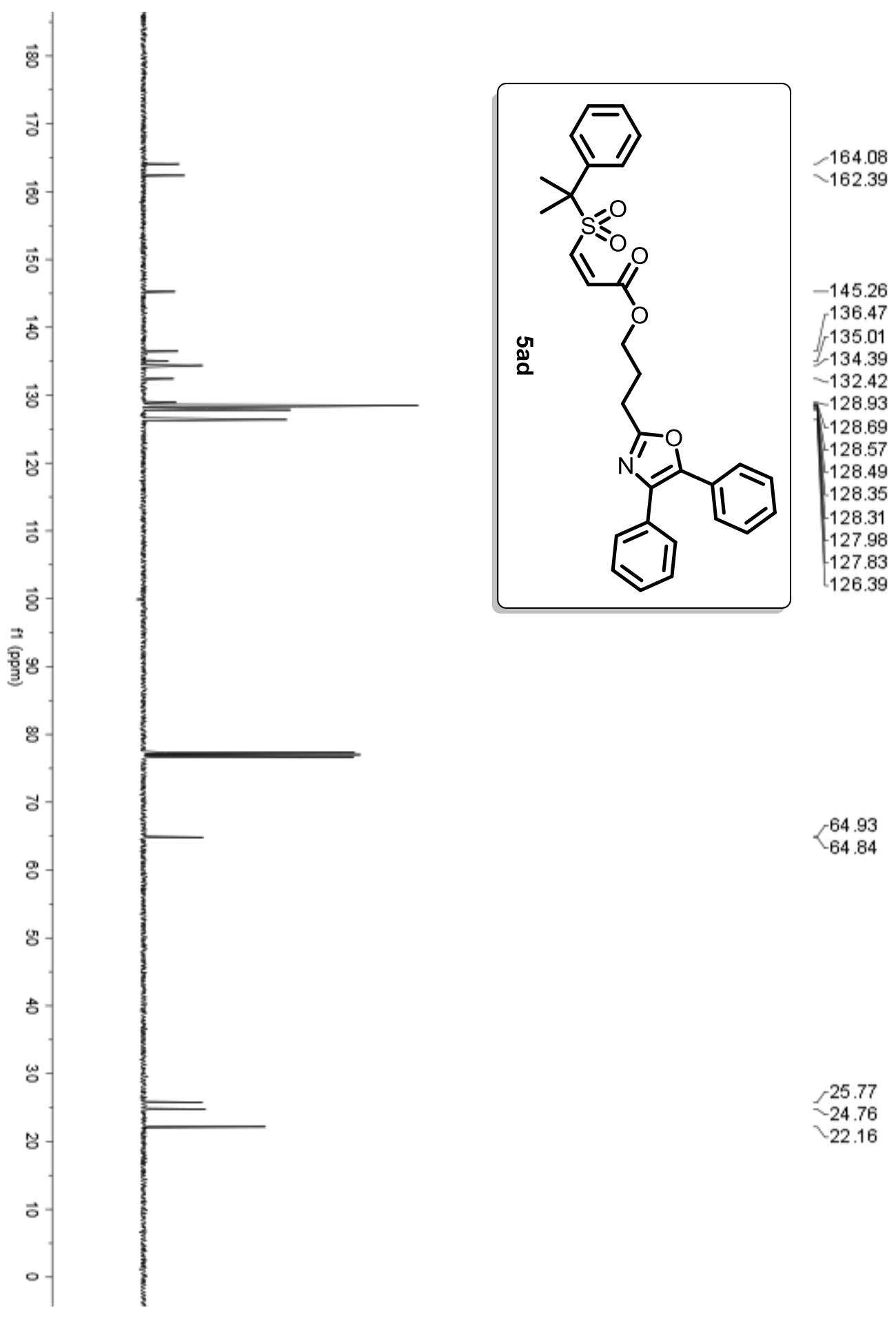


${ }^{1} \mathbf{H}$ NMR $\left(400 \mathrm{MHz}, \mathrm{CDCl}_{3}\right)$

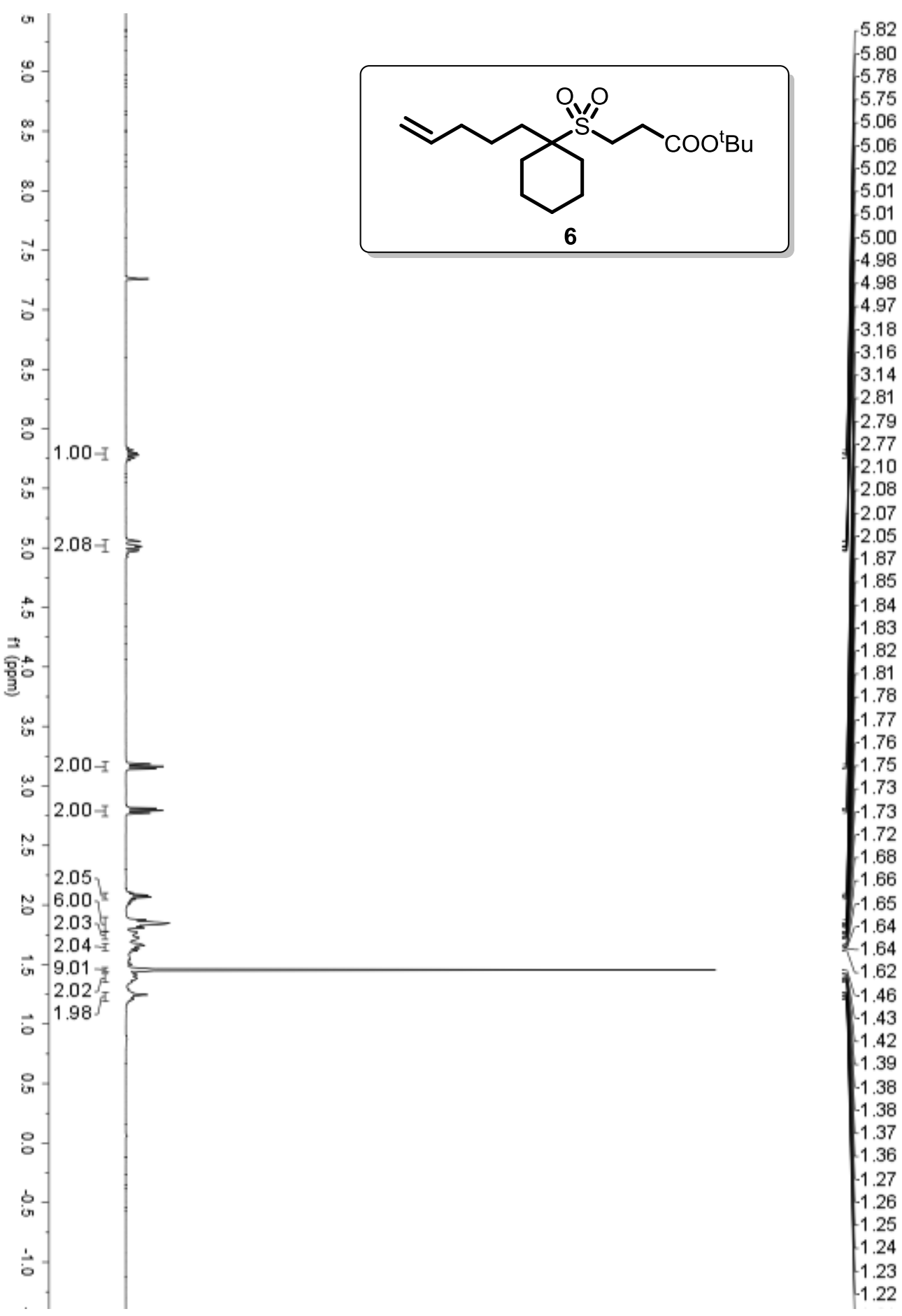


${ }^{13} \mathrm{C}$ NMR $\left(100 \mathrm{MHz}, \mathrm{CDCl}_{3}\right)$
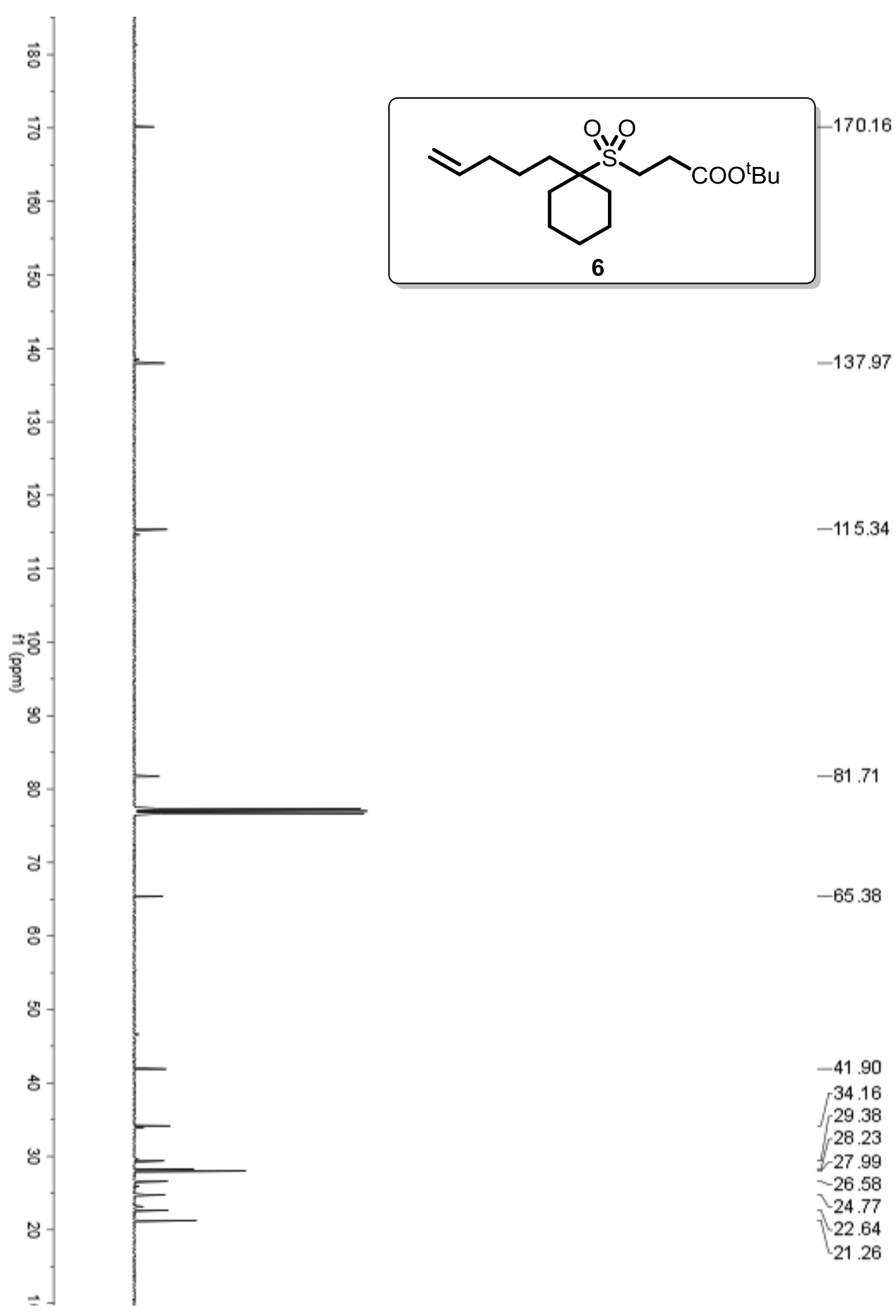

$-115.34$

$-81.71$

$-65.38$

$-41.90$

34.16

. 29.38

28.23

$-27.99$

$-26.58$

$-24.77$

ㄱ. 22.64

21.26 
${ }^{1} \mathbf{H}$ NMR $\left(400 \mathrm{MHz}, \mathrm{CDCl}_{3}\right)$

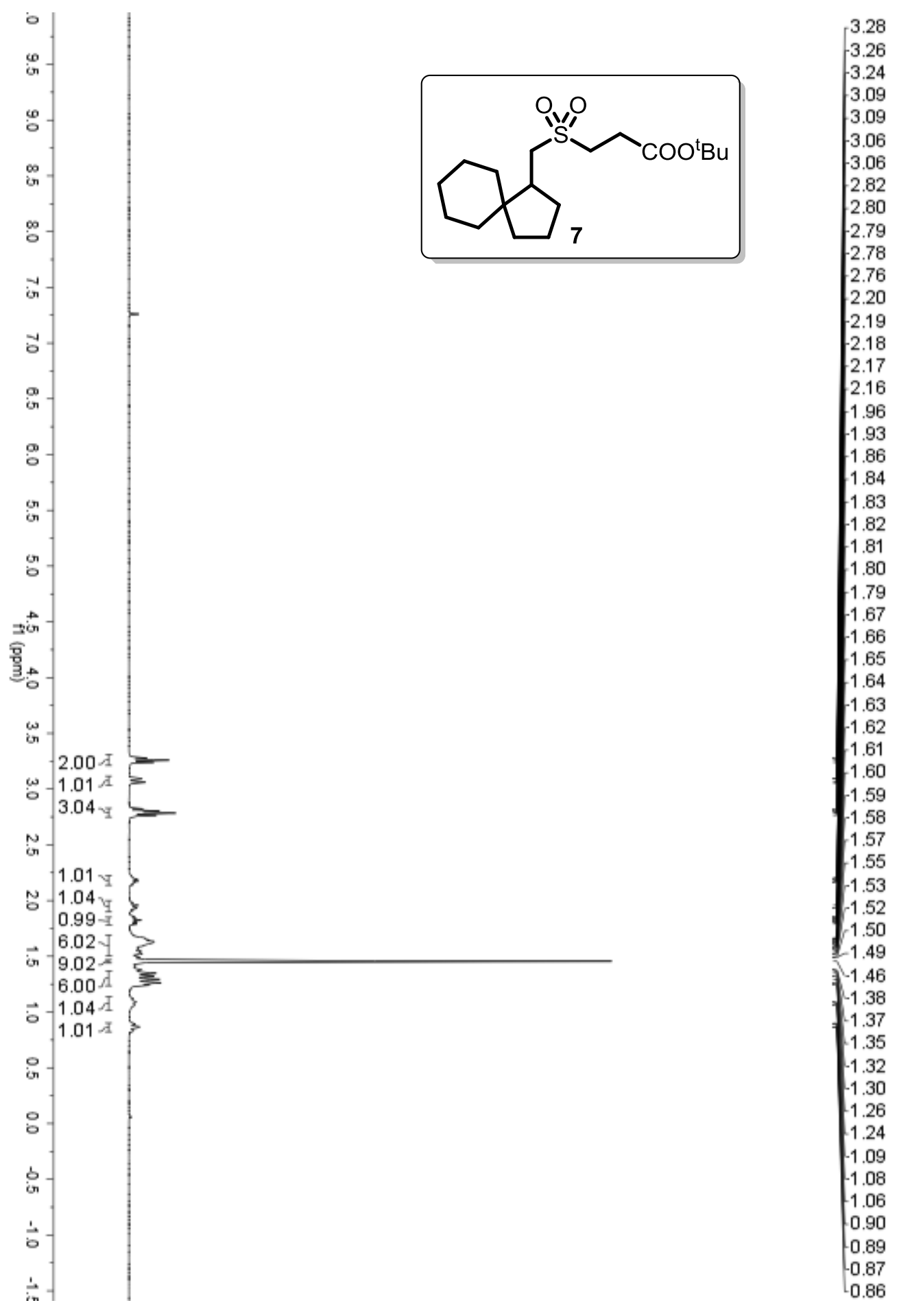


${ }^{13} \mathrm{C}$ NMR $\left(100 \mathrm{MHz}, \mathrm{CDCl}_{3}\right)$
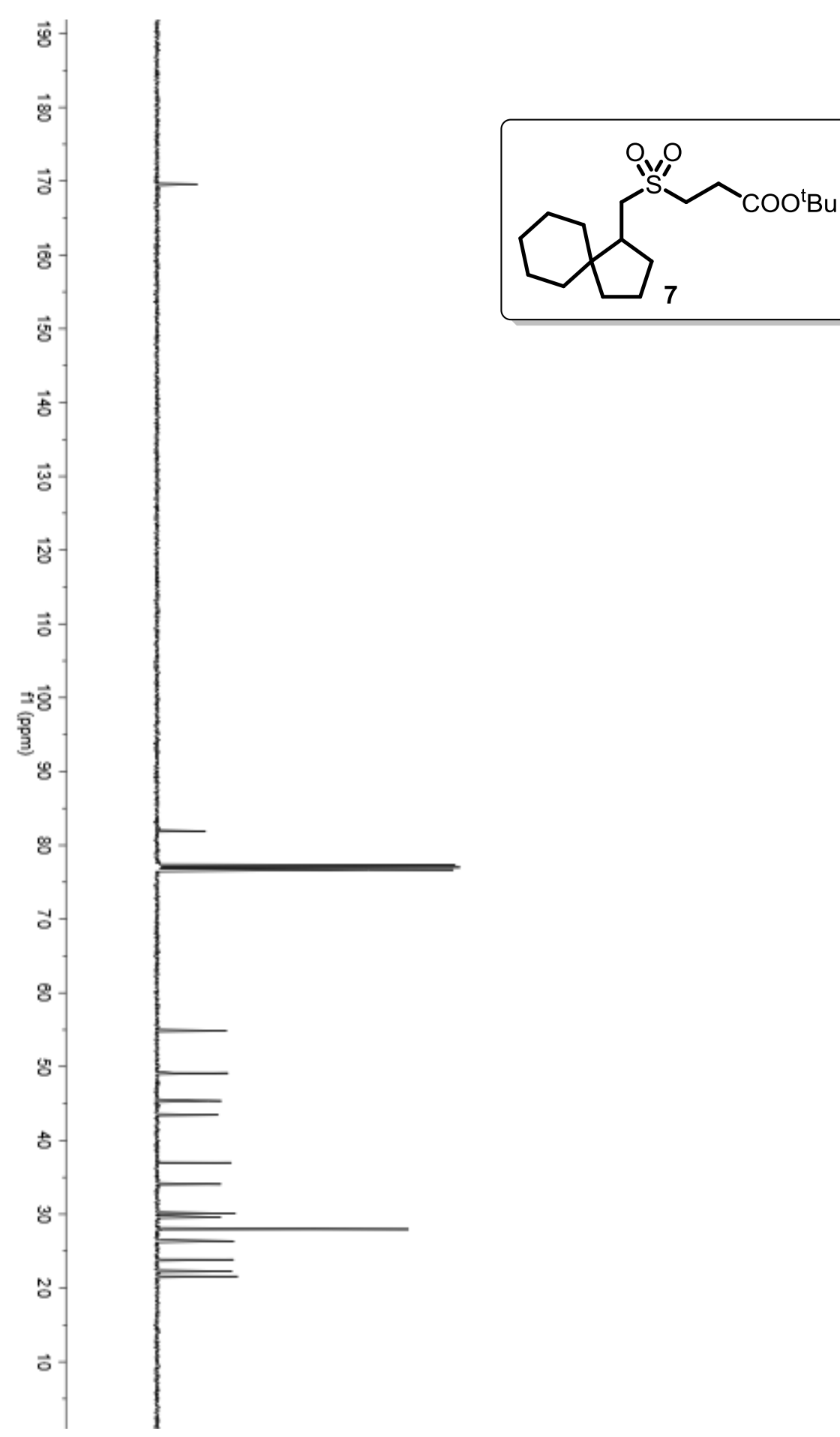
DEPT $135\left(100 \mathrm{MHz}, \mathrm{CDCl}_{3}\right)$

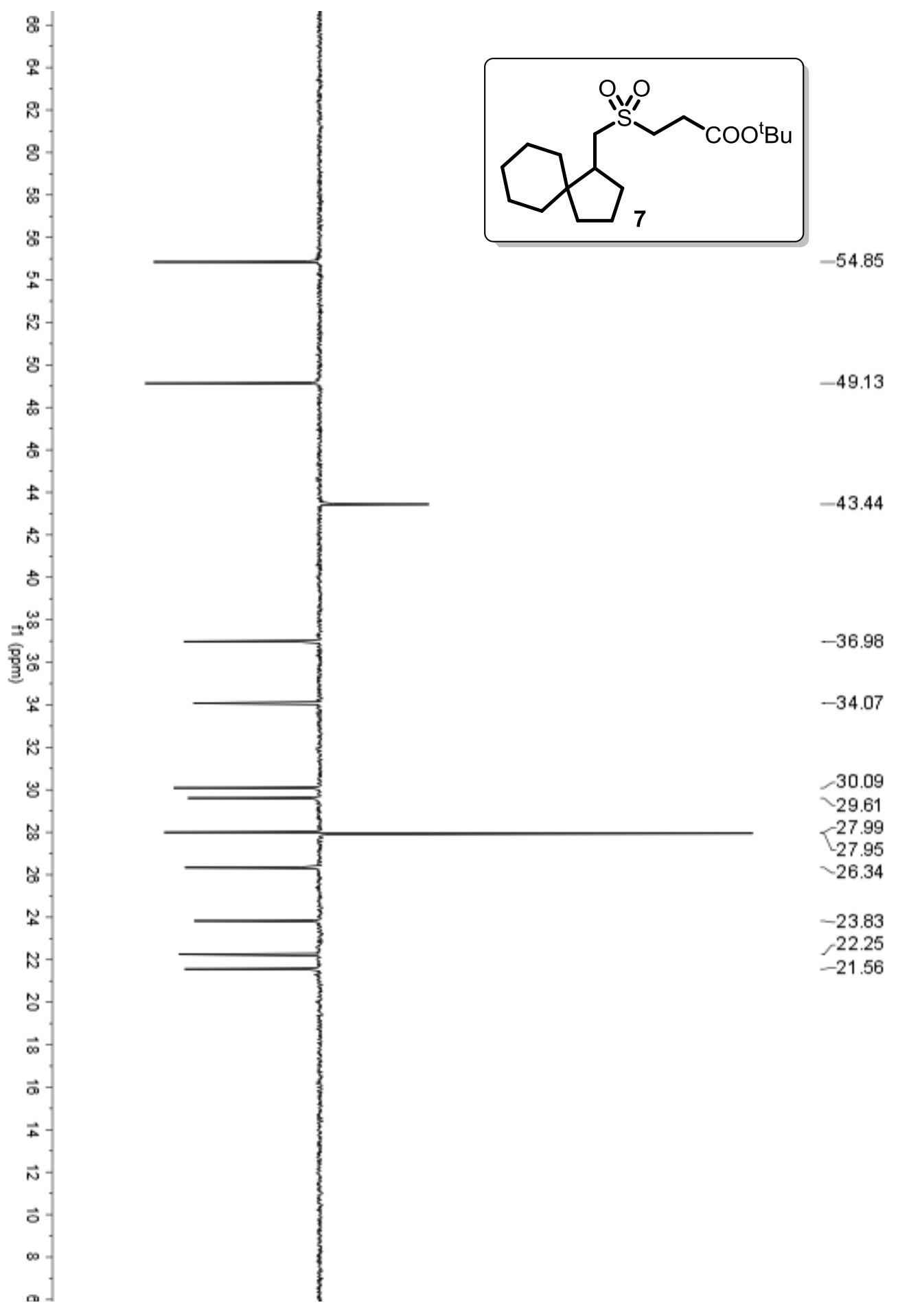




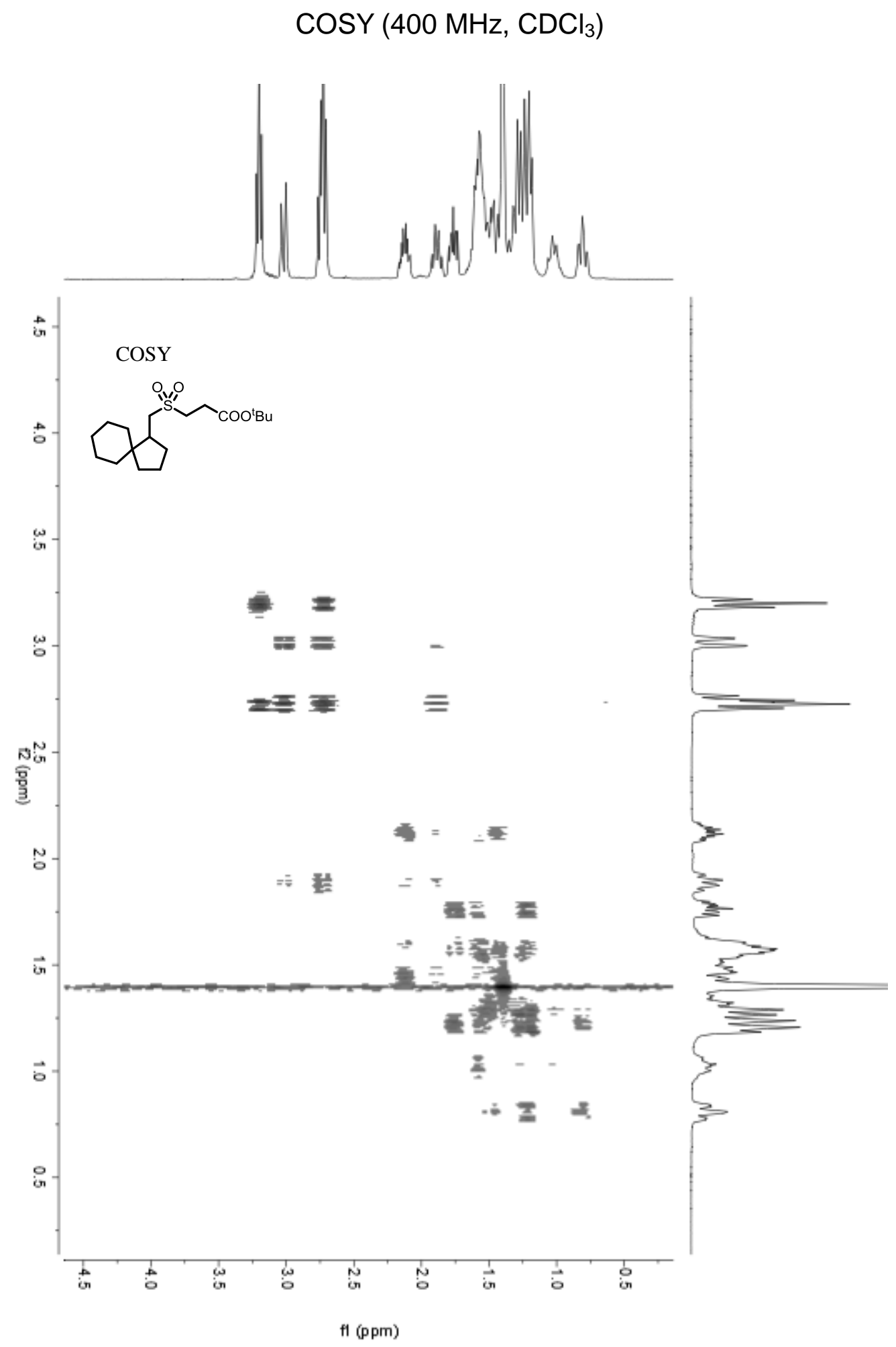


HSQC $\left(400 \mathrm{MHz}, \mathrm{CDCl}_{3}\right)$

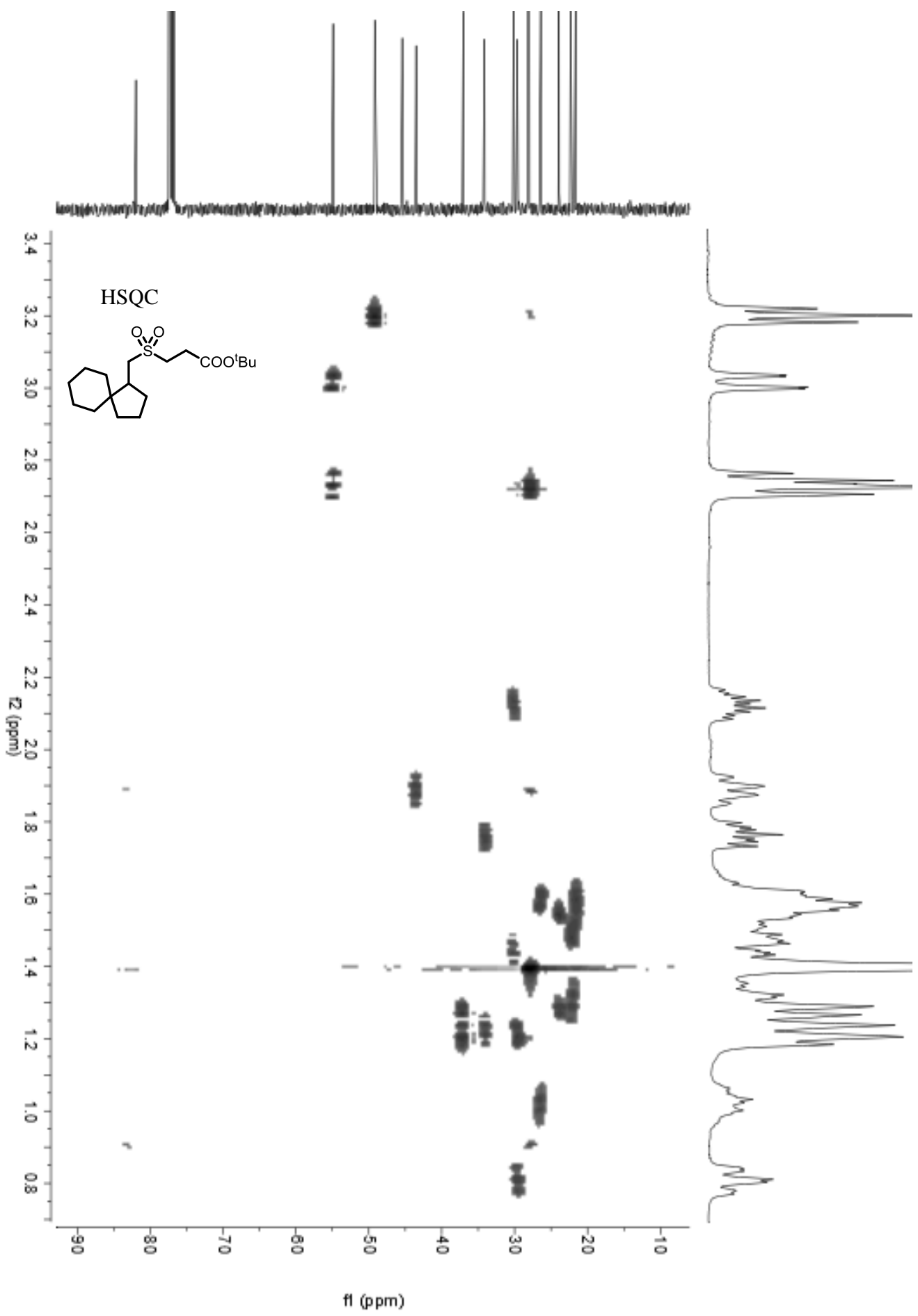


$\mathrm{HMBC}\left(400 \mathrm{MHz}, \mathrm{CDCl}_{3}\right)$

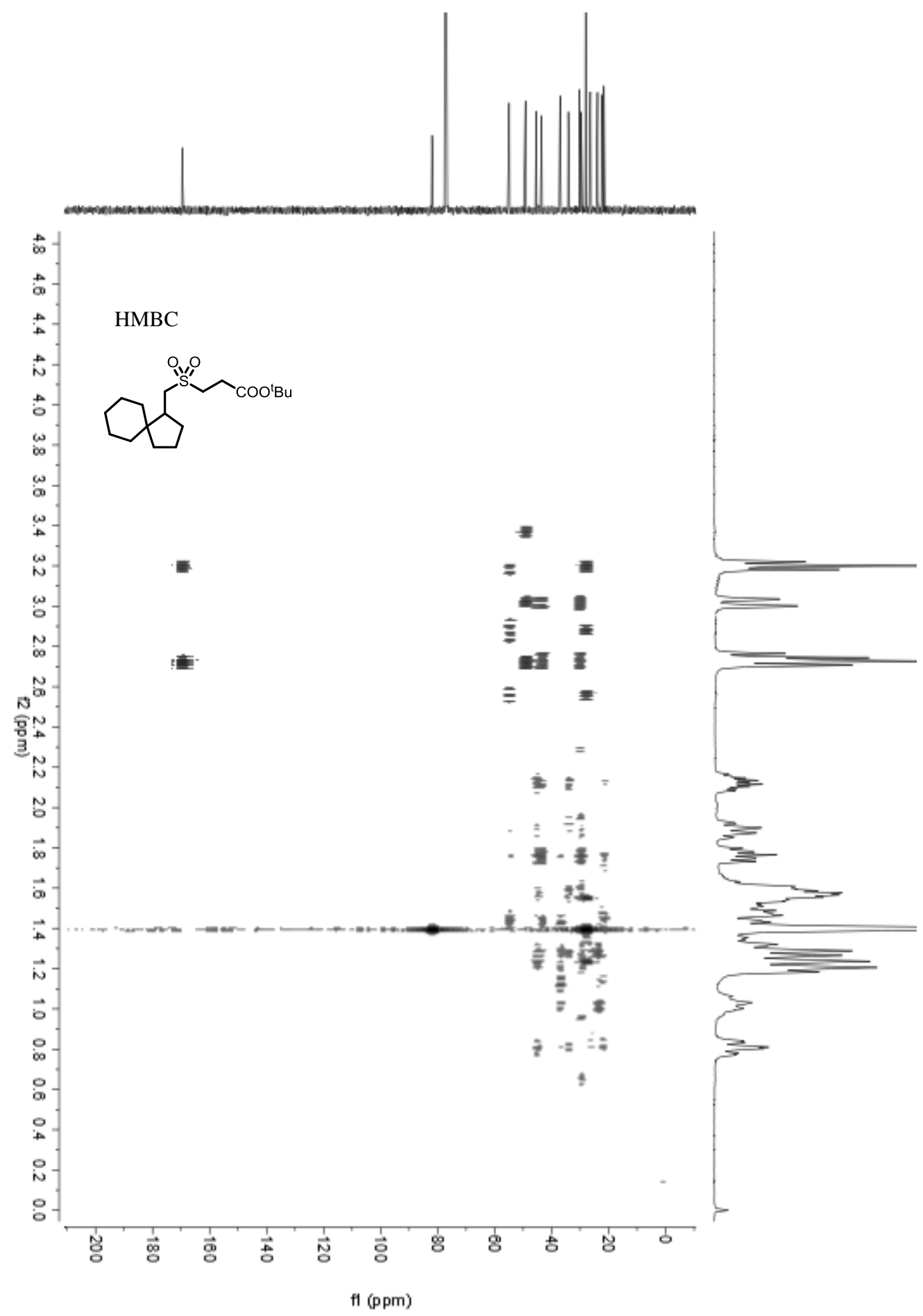


NOESY $\left(400 \mathrm{MHz}, \mathrm{CDCl}_{3}\right)$

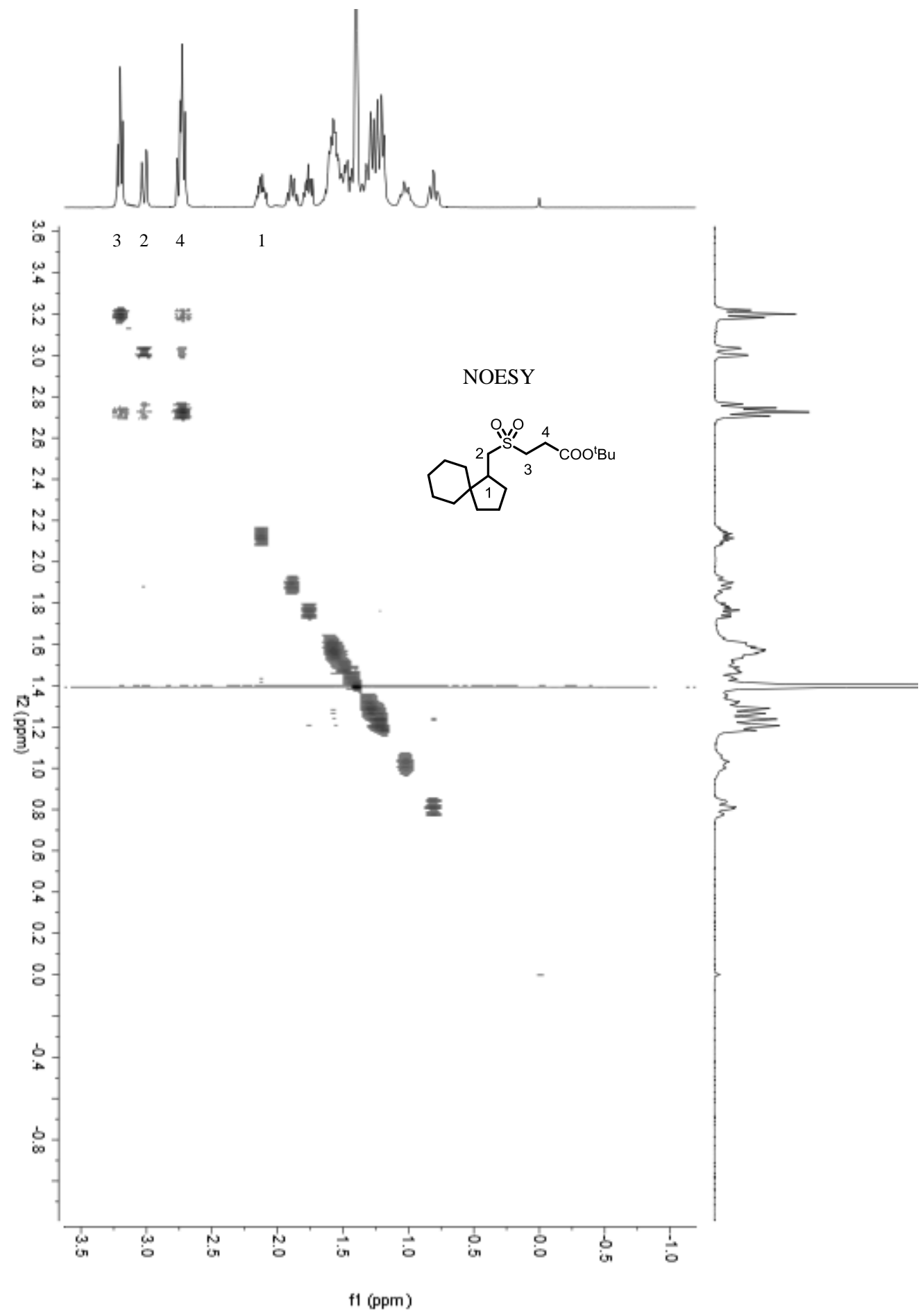


${ }^{1} \mathbf{H}$ NMR $\left(400 \mathrm{MHz}, \mathrm{CDCl}_{3}\right)$

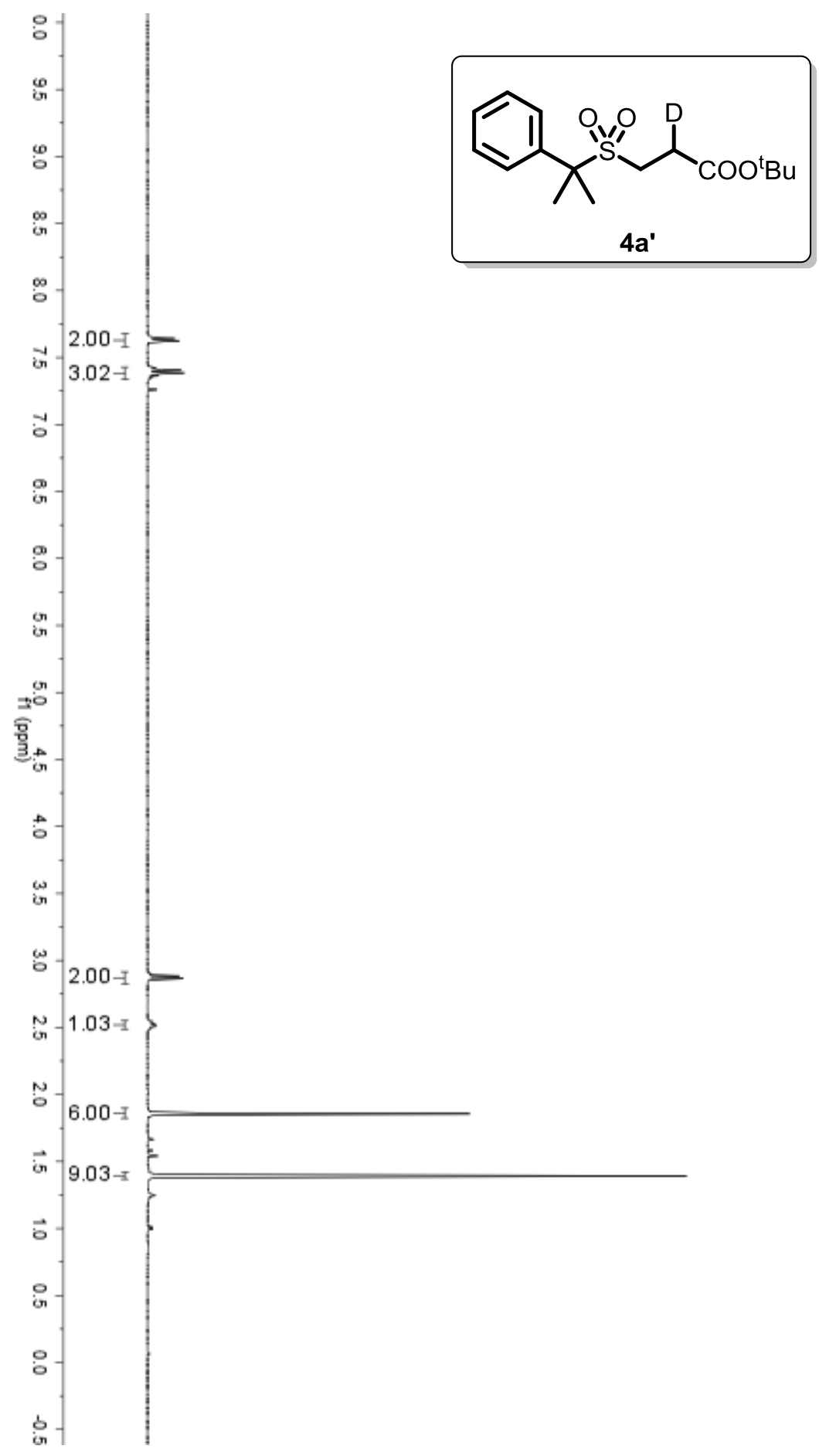


${ }^{13} \mathrm{C}$ NMR $\left(100 \mathrm{MHz}, \mathrm{CDCl}_{3}\right)$

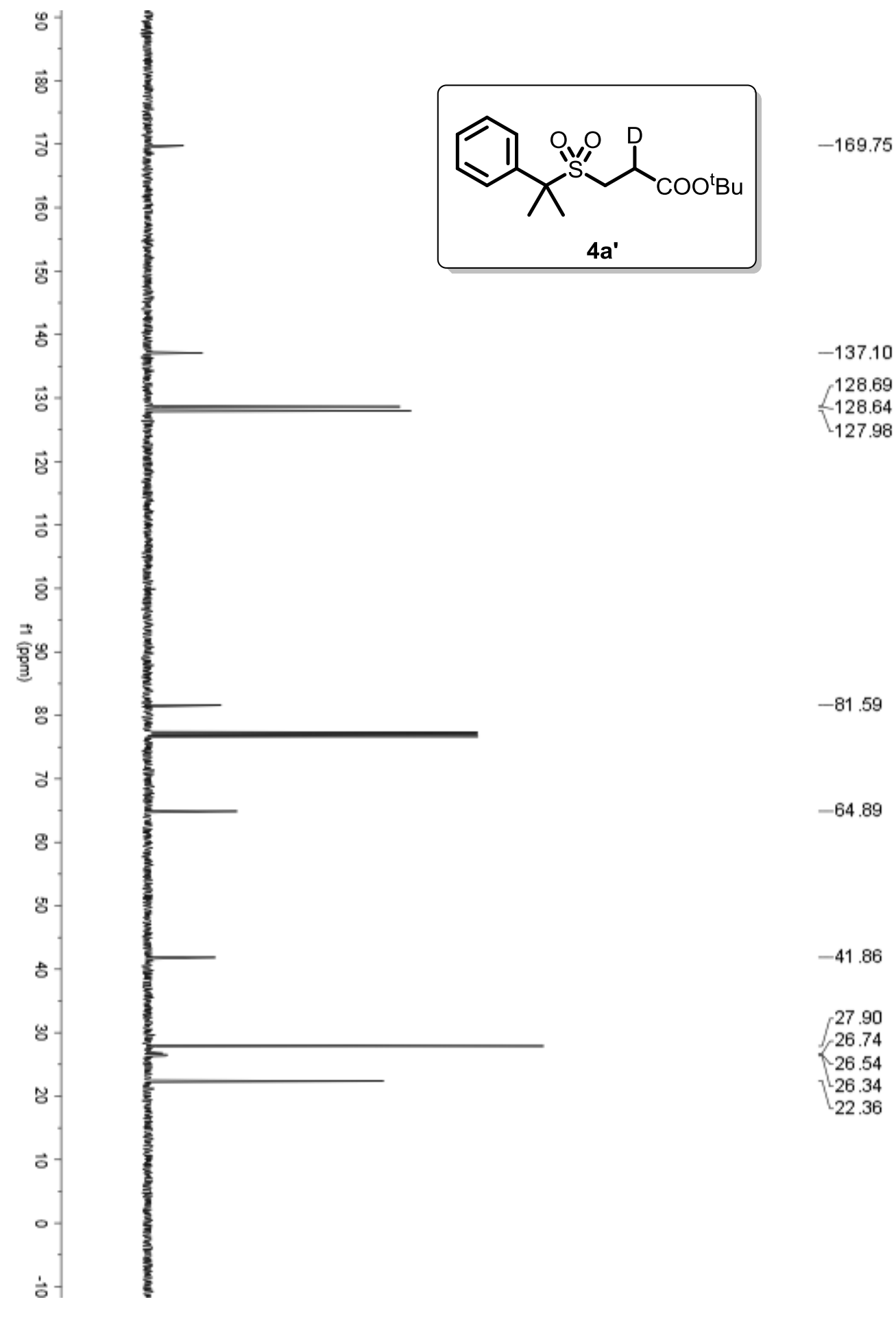


${ }^{1} \mathbf{H}$ NMR $\left(400 \mathrm{MHz}, \mathrm{CDCl}_{3}\right)$

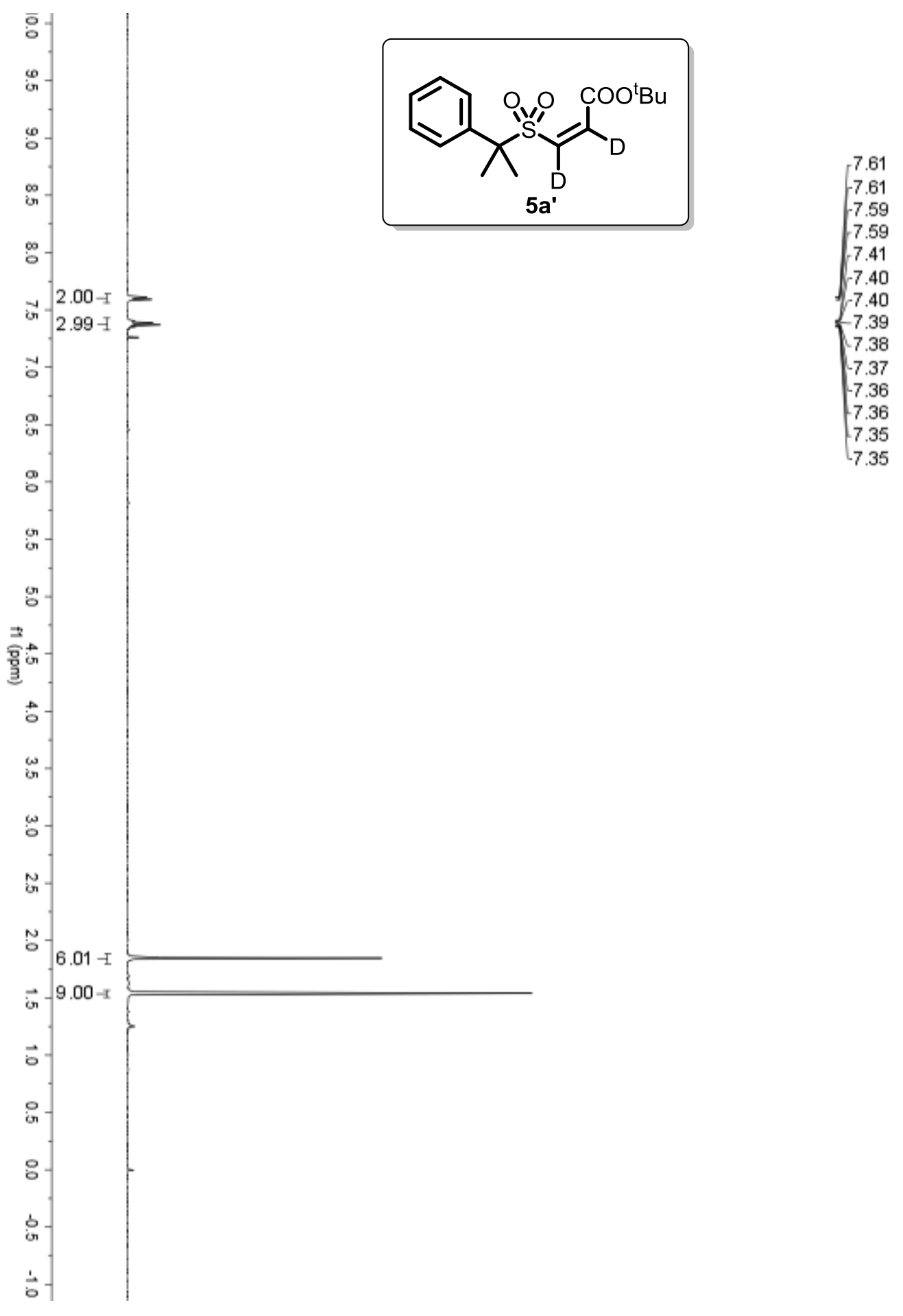


${ }^{13}$ C NMR $\left(100 \mathrm{MHz}, \mathrm{CDCl}_{3}\right)$

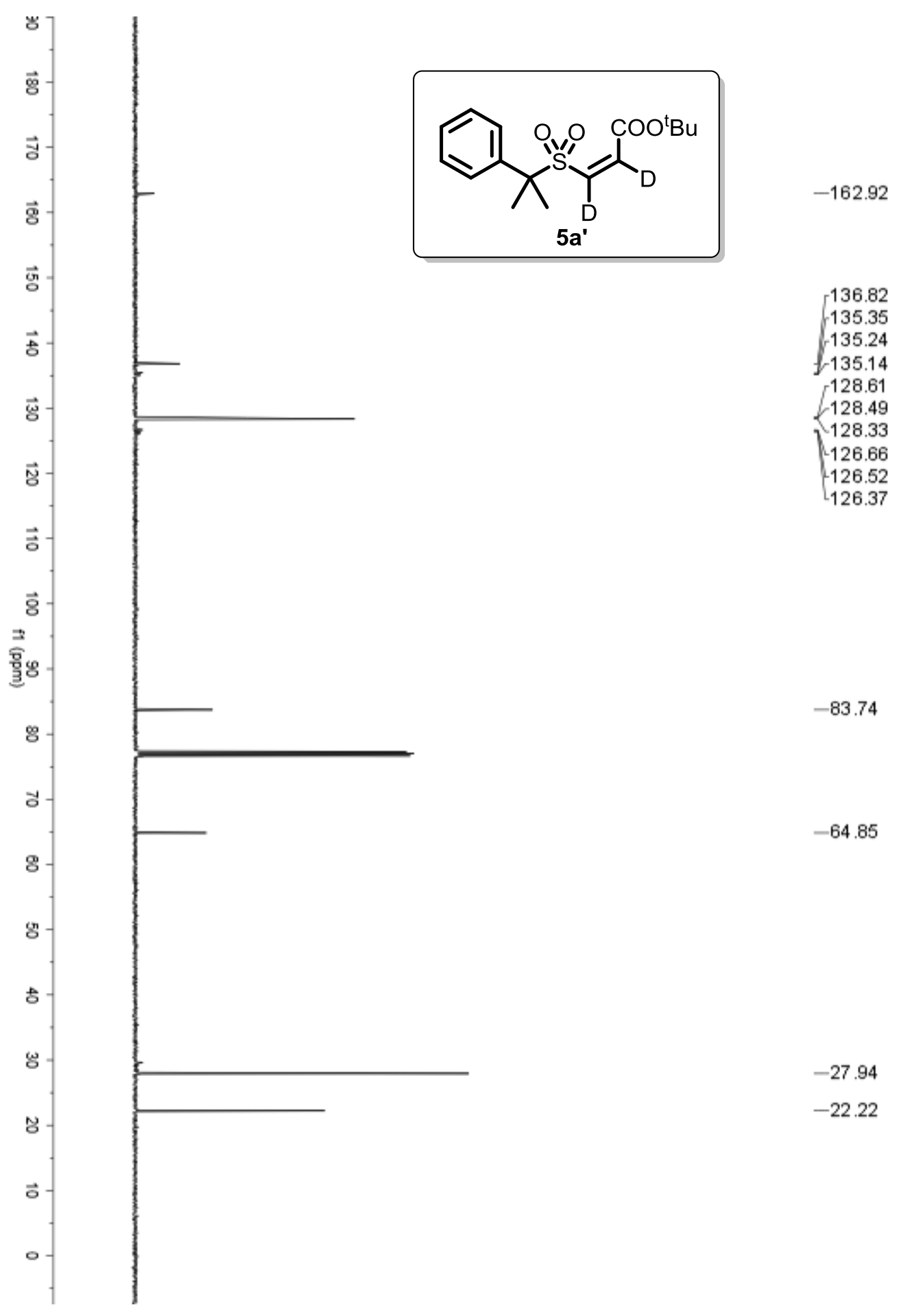


${ }^{1} \mathbf{H}$ NMR $\left(400 \mathrm{MHz}, \mathrm{CDCl}_{3}\right)$

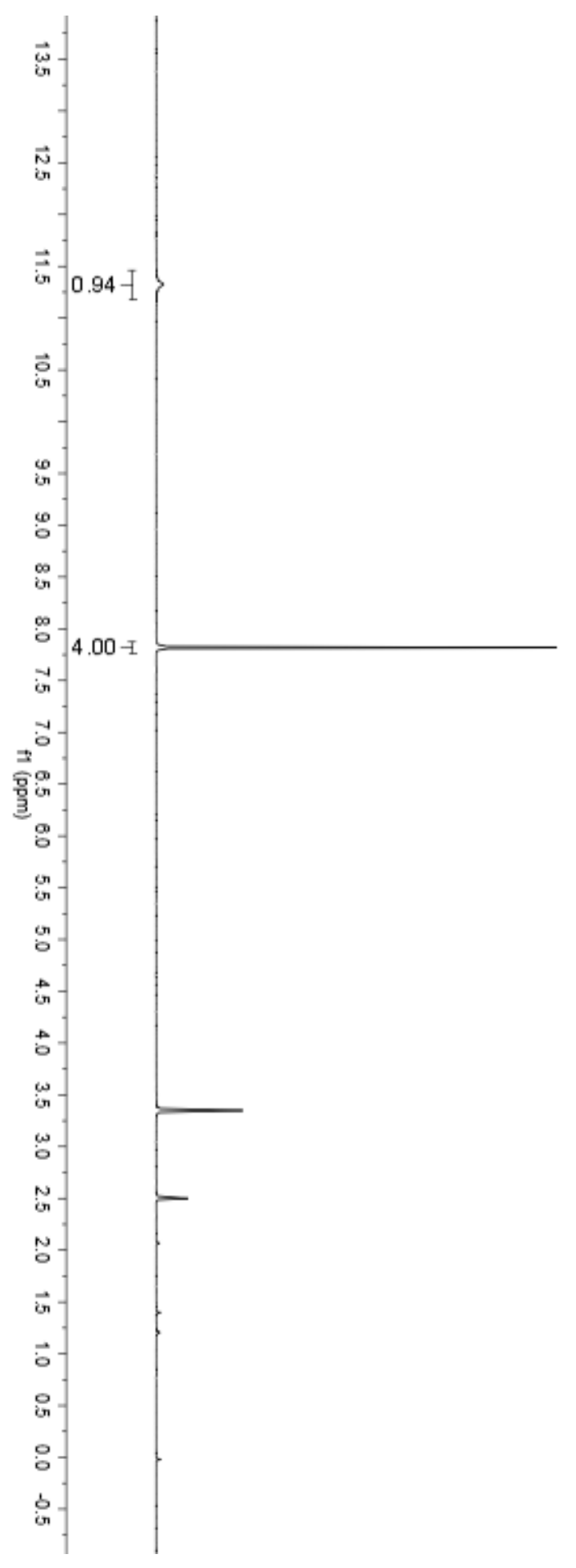


${ }^{13} \mathrm{C}$ NMR $\left(100 \mathrm{MHz}, \mathrm{CDCl}_{3}\right)$

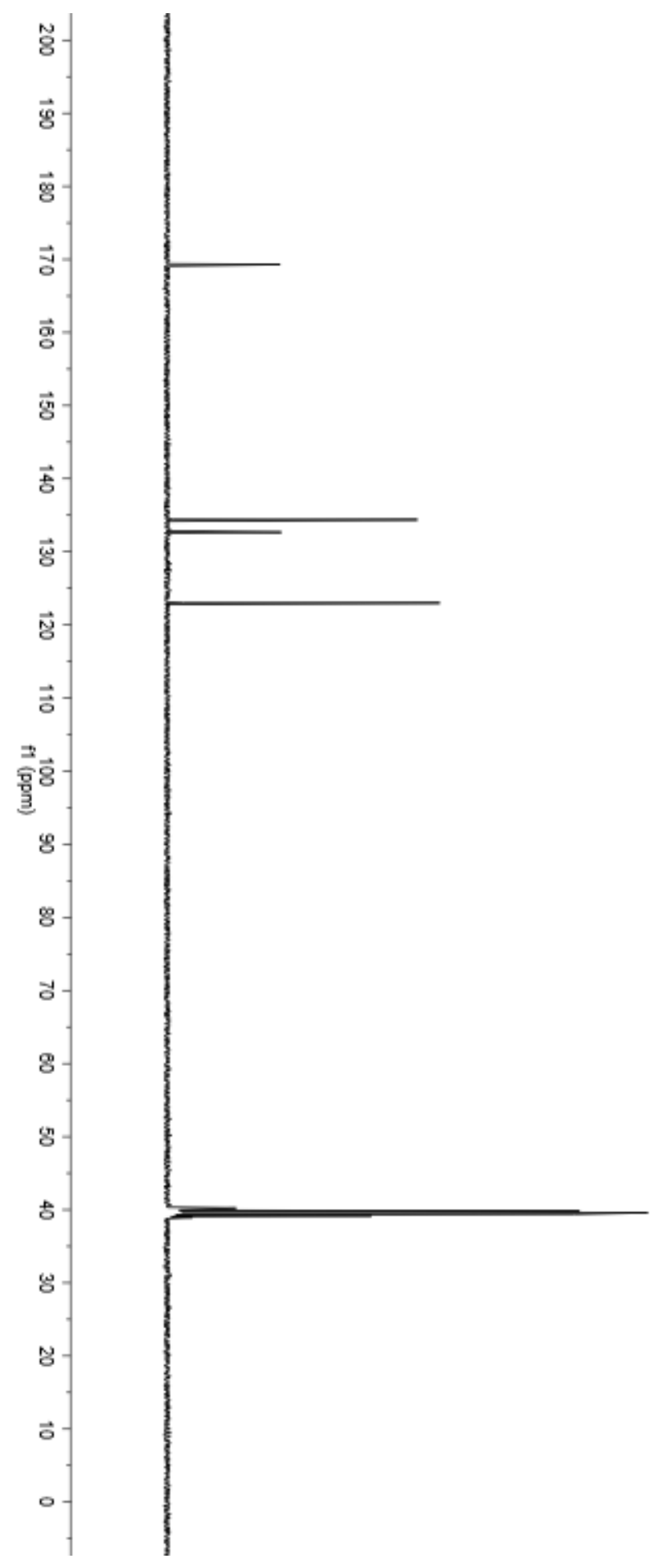

